Christophe Barlieb, Lidia Gasperoni (Hg.)

\title{
Media Agency
}

Neue Ansätze zur Medialität in der Architektur 
Christophe Barlieb, Lidia Gasperoni (Hg.)

Media Agency

Architektur Denken 10 


\section{Architektur Denken}

Architekturtheorie und Ästhetik

Herausgeber: Jörg H. Gleiter, Berlin

Beirat: Nathalie Bredella, Berlin

Tim Kammasch, Bern

Dietrich Neumann, Providence (RI) 
Christophe Barlieb, Lidia Gasperoni (Hg.)

\section{Media Agency}

Neue Ansätze zur Medialität in der Architektur

[transcript] 
Diese Publikation wurde aus dem Open-Access-Publikationsfonds der Technischen Universität Berlin unterstützt.

Bibliografische Information der Deutschen Nationalbibliothek

Die Deutsche Nationalbibliothek verzeichnet diese Publikation in der Deutschen Nationalbibliografie; detaillierte bibliografische Daten sind im Internet über $h t t p s: / / p o r t a l . d n b . d e /$ abrufbar.

Dieses Werk ist lizenziert unter der Creative Commons AttributionShareAlike 4.0 Lizenz (BY-SA). Diese Lizenz erlaubt unter Voraussetzung der Namensnennung des Urhebers die Bearbeitung, Vervielfältigung und Verbreitung des Materials in jedem Format oder Medium für beliebige Zwecke, auch kommerziell, sofern der neu entstandene Text unter derselben Lizenz wie das Original verbreitet wird.

Lizenz-Text: $h$ ttps://creativecommons.org/licenses/by-sa/4.0/deed.de

Die Bedingungen der Creative-Commons-Lizenz gelten nur für Originalmaterial. Die Wiederverwendung von Material aus anderen Quellen (gekennzeichnet mit Quellenangabe) wie z.B. Schaubilder, Abbildungen, Fotos und Textauszüge erfordert ggf. weitere Nutzungsgenehmigungen durch den jeweiligen Rechteinhaber.

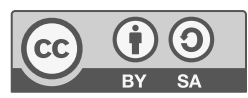

Erschienen 2020 im transcript Verlag, Bielefeld

(c) Christophe Barlieb, Lidia Gasperoni (Hg.)

Lektorat: Alexander Stumm

Korrektorat: Sandra Meireis

Gestaltung und Satz: Philipp Heinlein, Berlin

Druck: Majuskel Medienproduktion $\mathrm{GmbH}$, Wetzlar

ISBN (Print): 978-3-8376-4874-4

ISBN (PDF): 978-3-8394-4874-8

https://doi.org/10.14361/9783839448748

Gedruckt auf alterungsbeständigem Papier mit chlorfrei gebleichtem Zellstoff. Besuchen Sie uns im Internet: www.transcript-verlag.de Unsere aktuelle Vorschau finden Sie unter: www.transcript-verlag.de/vorschau-download 


\section{Inhalt}

7 Christophe Barlieb, Lidia Gasperoni Einleitung

13 Lidia Gasperoni „Nicht alle Mittel sind auch Medien". Die Media-Agency-Theorie

\section{Ana García Varas}

Die Debatte über Agency in der Architekturtheorie

52 Kassandra Nakas Manifeste, Diskurse, Chatter Sprache als Medium der Architektur

69 Carolin Höfler Über die Wirkmacht der Linie: Hadids Kalligramme

100 Anna Hougaard Skizzen von Diagrammen und Diagramme von Skizzen

120 Philipp Reinfeld Fotografie und Entwerfen

145 Matthias Ballestrem Experimentelles Entwerfen: Methoden des kontrollierten Kontrollverlusts

161 Norbert Palz

Architektonischer Entwurf und differenzierte Mediennutzung

179 Corneel Cannaerts Hacking Agency: Digitale Fabrikation als Entwurfsmedium

197 Christophe Barlieb Cybercraft: Das neue Paradigma

217 Bildnachweise

221 Autorlnnen 

Christophe Barlieb, Lidia Gasperoni

\section{Einleitung}

Architektur entsteht durch Medien des Entwerfens. Dieser Sammelband ist der Untersuchung der generativen Funktion von Medien in der Architektur gewidmet; diese aktive Funktion wird als Media Agency bezeichnet. Ziel der Beiträge ist es, die Funktion von Medien in Entwurfsprozessen zu überdenken und neu zu bewerten.

Der Fokus liegt dabei auf der Reziprozität und Verschränkung medialer Praktiken - besonders dort, wo sie komplexe, ineinandergreifende Systeme bilden. In Hinblick auf die Architektur heißt das, nicht nur Erscheinungsformen wie Material, Form, Figur, Struktur, Oberflächenbeschaffenheit in den Blick zu nehmen, sondern auch die Art ihrer Generierung. Architekturmedien werden somit in ihrem generativen Potential und in ihrer agentiellen Kraft beschrieben. Die Theorie der agentiellen Funktion von Medien verschiebt den Fokus des Entwerfens von einer rein auktorialen Perspektive, aus der Medien als bloße Instrumente des schaffenden Subjekts erscheinen, hin zu einer prozesshaften Dimension, in der Medien den Entwurfsprozess entscheidend prägen oder ihn überhaupt erst generieren. Dabei werden auch historische Momente der Architekturdarstellung - wie etwa Albertis Verwendung der Perspektive, Descartes' Visualisierung des optischen Raums, Leibniz' Binärsystem und Monges Darstellende Geometrie - herangezogen, um sie von einem medialen Standpunkt aus neu zu deuten, nachdem traditionell-repräsentationalistische Vorstellungen im vergangenen Jahrhundert zunehmend in die Kritik geraten sind. In der Folge dieser Kritik ist ein freieres Experimentieren mit Medien durch Künstler und Architektinnen zu beobachten, das sich mehr und mehr vom Ideal einer repräsentationalen Architektur entfernt. 
Die Krise der Repräsentation charakterisiert die Architektur bis in die Postmoderne und die Digitale Revolution, wobei letztere zwei Seiten aufweist: Während digitale Medien einerseits zur Manipulation und zur Erzeugung neuer Formen eingesetzt werden, ist ihre Verwendung zur Simulation der Realität weiterhin größtenteils repräsentationalistisch und schlicht nachbildend.

Hybridisierung der Medien Entwerfende im Sinne Albertis verlangen nach neuen Medien. Die vergangenen Jahrzehnte zeugen vom rasanten Wandel der Gesellschaft: Wir erleben, wie Medien kollidieren, sich verzahnen und Hybride bilden. Neben der Medienbildung unterstützen Medien Denk-, Wahrnehmungs- und Handlungsmodi, die in Sozialen Medien, Crowdsourcing und dergleichen selbstverständlich sind und unseren Alltag beeinflussen.

Wir stehen davor, das volle Ausmaß eines ,Hybridzeitalters ‘ zu erleben, in dem analoge und digitale Bereiche quasi nahtlos ineinandergreifen. Doch während sich diese Verzahnung vollzieht, erscheinen unsere Methoden und Modelle, die sie adressieren, zunehmend obsolet. Unsere raumentwerfenden Praktiken bleiben weitgehend Projektionen auf zweidimensionale Flächen. Wir haben, anders gewendet, eine Transformation vom Analogen zum Digitalen erlebt, ohne dabei die medialen und räumlichen Auswirkungen dieses Wandels hinreichend zu bedenken. Dies ist am deutlichsten an Computer-Aided Design (CAD) zu sehen. Obwohl sich CAD in den letzten dreißig Jahren stark weiterentwickelt hat, funktioniert es weiterhin mit Projektionen auf zweidimensionalen Flächen, d.h. auf Displays. Während zeitgenössische digitale Modellierungsprozesse gewissermaßen greifbar sind, ist die physische Realisierung von Modellen weiterhin langwierig, teuer. Die Vorstellung eines direkten physischen Kontakts mit Medien, die den Denk- und Herstellungsprozess essentiell prägen ist somit im Wandel. Stattdessen werden zusätzliche Dispositive zur Übersetzung eingesetzt. Aktuelle Trends forcieren die Modellierung quasi in Echtzeit in Computerumgebungen, wobei die gleichzeitige, materielle Produktion mit Fertigungsmaschinen angestrebt wird, was in Zukunft Standard sein dürfte. Idealerweise vermischen Medien die analogen und digitalen Bereiche auf einzigartige Weise: Schöpfer, Modelle, Materialien und Maschinen sind in einem Prozess vereint. Langfristig können Maschinen entstehen, die diese Prozesse selbst aushandeln. 
Im digitalen Zeitalter ist also die Frage der Repräsentation durch Medien höchst aktuell: Stellen digitale Entwurfsmethoden eine spezifische Verwendung von technischen Dispositiven dar? Sind sie Medien? Und wie verändern sie die zeitgenössische Architektur, die bald maschinell erzeugt werden kann? Was ist dabei die Rolle einerseits der Entwerfer und andererseits der Medien, wenn letztere nicht nur als Instrumente zu verstehen sind? Gibt es analoge Medien oder analoge Gebrauchsweisen von Medien, die sich dem Digitalen vollständig entziehen?

Medien sind zwar allgemein bekannt, jedoch begrifflich weiterhin sehr vage gefasst. Der Anspruch des vorliegenden Bandes besteht unter diesen Vorzeichen gerade darin, die generative Verwendung von Medien im architektonischen Entwerfen neu zu beleuchten. Die Beiträge versuchen die Medien dorthin zu rücken, wo diese sein sollten: Medien sollten im Zentrum des Denkens und der Herstellung der Architektur stehen, und nicht nur als ein Mittel zur Darstellung, Simulation und Bearbeitung von Architektur begriffen werden. Einige der Fragen, mit denen wir uns befassen, lauten: Wie können wir über die illusorische Repräsentation der Wirklichkeit in der Digitalen Revolution hinausgehen? Was sind die performativen Qualitäten von Medien? Wie können wir einer neuen Social-Media-Generation von Architekten und Architektinnen beibringen, (analoge, digitale und hybride) Medien in der Architektur zu adressieren, zu bewerten und einzusetzen?

Inhalt des Bandes Der Band vereint verschiedene Ansätze, die mit der Medialität räumlicher Wahrnehmung experimentieren, um neue Potenziale beim Denken, Skizzieren, Modellieren und Verwirklichen von Architekturen zu erschließen. Die einzelnen Beiträge sind das Ergebnis einer Forschungsinitiative, die 20 I 5 im von Christophe Barlieb geleiteten Fachgebiet für Architekturdarstellung und -gestaltung der TU Berlin entstanden ist und in den Jahren Zulauf erhalten hat durch Forschende und Entwerfende, die neue Zugänge zu Medien in der Architektur erforschen. Die Autoren und Autorinnen des Bandes setzen sich auf unterschiedliche Weise mit Medienbedingungen der zeitgenössischen Architektur auseinander - angefangen bei symbolisch-geschriebenen Sprachen über logische Computersprachen, Skizziertechniken, diagrammatische Zeichnungen, bildbasierte Architekturen, digitale Modell- und Materialpraktiken bis hin zu Hacking-Prozeduren und Medienhy- 
briden. Medien werden dabei primär als Prozesse oder inhärente Ausdrucksformen verstanden, die neue Gestaltungspotenziale erschließen.

Die ersten Beiträge sind den Grundlagen der Media-AgencyTheorie gewidmet. Lidia Gasperoni geht es um die philosophischen Implikationen des Begriffs der Media Agency sowie um dessen Anwendung im architektonischen Entwerfen. Im zweiten Aufsatz analysiert Ana García Vargas den Begriff der Agency in der Architekturtheorie.

Die weiteren Beiträge befassen sich mit spezifischen Medien als generative Praktiken des Entwerfens. Es werden verschiedene Medienmethoden und -modelle vorgestellt, die geeignet erscheinen, auch in der architektonischen Lehre angewandt $\mathrm{zu}$ werden, um durch eine entwerferisch-generative Reflexion neue Architekturen zu erschließen. Insgesamt behandelt der Sammelband auf diese Weise die verschiedensten medialen Sprachen der Architektur. Es geht um spezifische Entwurfsmedien: von analogen bis hin zu zeitgenössischen, hybriden Medien, die aus Sicht der Media Agency behandelt werden. Kassandra Nakas untersucht die Relevanz der Sprache in ihrer textuellen Form als Medium der Architektur, während Anna Hougaard und Carolin Höfler sich mit der diagrammatischen Verwendung der Zeichnung auseinandersetzen. Philipp Reinfeld analysiert die mediale Dimension der Fotografie in der Architektur. Matthias Ballestrem dagegen entwickelt eine eigene Definition des experimentellen Entwerfens, bei der die auf den ersten Blick kontradiktorische Idee eines kontrollierten Kontrollverlusts eine zentrale Rolle spielt.

Die abschließenden drei Aufsätze von Norbert Palz, Corneel Cannaerts und Christophe Barlieb beziehen sich auf digitale Medien und die Möglichkeiten ihrer Hybridisierung mit analogen Medien. Während Palz für eine differenzierte Mediennutzung plädiert, führt Cannaerts den Begriff des Hackings als einer Fähigkeit ein, Medien nicht nur zu verwenden, sondern neu zu programmieren und zu adaptieren. Barlieb bezieht sich auf den Begriff des Cybercraft als Weiterentwicklung von Handcraft und Digitalcraft sowie auf die hybride Veränderung einer zugleich analogen wie digitalen Architektur.

Im Ganzen zielt der Band somit auf die theoretische Grundlegung der Media Agency und auf ihre mögliche Anwendung in der architektonischen Praxis und Lehre. Die Media-Agency-Theorie 
könnte - wie traditionell die Darstellende Geometrie - zur Grundlage einer architektonischen Propädeutik werden, die an Universitäten bislang hauptsächlich auf die architektonische Repräsentation und die räumliche Kognition fokussiert, um die für die Architekturpraxis notwendige räumliche Erfahrung zu trainieren. Der Begriff der Media Agency hingegen erweitert die räumliche Kognition von Architekturstudierenden, indem er die Aufmerksamkeit auf das generative Entschlüsseln, das hybride Kodieren und die symbolische Vermittlung von Architekturmedien richtet. Medien erscheinen aus dieser Perspektive als Entwurfsgeneratoren, als Akteure und weniger als Repräsentationswerkzeuge.

Für die Fertigstellung dieses Bandes ist Jörg Gleiter zu danken, der diesen Band in die Reihe ArchitekturDenken aufgenommen hat und das Publikationsprojekt vielfältig unterstützt hat. Auch dem Universitätsverlag der TU Berlin ist zu danken, der mit seinem Publikationsfonds-Programm den Großteil der Kosten für diese Publikation übernommen und deren Realisierung als Open-accessPublikation ermöglicht hat. Den Mitarbeitern und Mitarbeiterinnen des transcript Verlags ist für ihre kompetente und achtsame Begleitung zu danken. An Julian Franke geht unser Dank für die Übernahme einiger Übersetzungen englischer Texte und an Alexander Stumm und Sandra Meireis für das Lektorat. Unser Dank gilt auch den Autorinnen und Autoren, die in den letzten Jahren an diesen Band geglaubt, seine Inhalte mitdiskutiert und ihn mit ihren Reflexionen bereichert haben. Schließlich ist unseren Familien für die langjährige Unterstützung dieses Projekts zu danken. 

Lidia Gasperoni

\section{„Nicht alle Mittel sind auch Medien". Die Media-Agency-Theorie}

John Dewey beschreibt in Kunst als Erfahrung zwei mögliche Formen der Zielsetzung: „Es gibt Ziele, die bloß ein erwünschtes Ende sind, und andere, die einen vorausgehenden Prozess vollenden".' Die erste Form entspricht einer rein instrumentellen Haltung den Zielen gegenüber, bei der der Prozess als bloßes Mittel zum Zweck verstanden wird. Die zweite dagegen impliziert eine Haltung, die dem Prozess gegenüber nicht indifferent ist; der Prozess ist dabei das Medium für das Erreichen des Ziels selbst. So kann Dewey feststellen: „Nicht alle Mittel sind auch Medien“, und weiter: „Es gibt zwei Arten von Mitteln. Die eine ist dem, was ausgeführt wird, äußerlich; die andere wird in die erzeugten Ergebnisse aufgenommen und bleibt ihnen immanent. [...] Die Mittel hören zu wirken auf, sobald das Ziel erreicht ist; in der Regel wäre man froh, das Ziel ohne Aufwendung der Mittel zu erreichen. Sie sind nur ein Gerüst. [...] Doch mit dem Moment, das wir Medien nennen, beziehen wir uns auf Mittel, die mit dem Ziel verbunden sind"..2

Einfach gesprochen geht es uns dabei wie beim Reisen: Wir können die Reise als rein instrumentellen Weg zum Ziel oder als prozesshaftes Moment des Reisens an sich betrachten. Wir können reisen, um ein Ziel zu erreichen und somit die Reise selbst als rein instrumentell begreifen; oder wir können irgendwohin reisen und gleichzeitig den Reiseweg als essentiellen Bestandteil der Reise ansehen. Im ersten Fall ist die Reise nur Mittel zum Zweck; im zweiten Fall ist die Reise Teil der Zielsetzung oder, wie Dewey sagt, „Mittel und Ziel fallen zusammen“.3

Diese zwei Formen des Reisens stehen exemplarisch für zwei verschiedene Lebensformen oder Haltungen, die sich auch auf 
den Kontext der Medialität übertragen lassen: Wir können die in Gestaltungs- und Darstellungsprozessen angewandten Medien wie Modelle, Bilder, Zeichnungen, Fotos, Texte - als bloße Instrumente begreifen und sie somit nur als Mittel zum Zweck des finalen Produkts betrachten; oder wir können sie als Bestandteil der Gestaltungsreise verstehen, durch die nicht nur ein Produkt zur Realisierung gelangt, sondern in deren Verlauf sich unsere Weise, architektonischen Raum wahrzunehmen und zu denken, überhaupt erst konstituiert. Während im ersten Fall Medien reine Werkzeuge sind, nehmen sie im zweiten Fall aktiven Anteil am Gestaltungsprozess und sind somit die Voraussetzung für unser Experimentieren mit den im Raum verborgenen Qualitäten. Dabei verändert sich auch unsere Einstellung gegenüber Zufällen: Aus Sicht der ersten (instrumentell-äußerlichen) Haltung erscheinen sie als bloße Hindernisse des geplanten Vorgehens, während sie aus Sicht der zweiten (performativ-immanenten) Haltung als Entdeckungsmomente gelten können, durch die der Prozess sich ändert, womit es zu neuen, zunächst ungeplanten Entwicklungen kommen kann. Solche unvorhersehbaren Entdeckungen sind stets Bestandteil von Gestaltungs- und Produktionsprozessen, wie viele Tätigkeiten an der Grenze zwischen Handwerk und Kunst - vom Weben bis zur digitalen Fabrikation - zeigen.

Um die so skizzierte, performative Haltung von einer bloß instrumentellen Verwendung von Werkzeugen auch terminologisch klar abzugrenzen, empfiehlt es sich, im ersten Fall von Medien zu sprechen, bei denen ihr performativer Gebrauch jeweils mitgedacht ist, während die traditionelle, instrumentelle Haltung dem Begriff des Mittels entspricht.

Letztlich geht es um die Haltung, die wir einnehmen. Und es geht gleichzeitig um eine bewusste Haltung, die wir den Mitteln (als rein instrumentellen Wegen) und den Medien (als performativen Wegen) gegenüber einnehmen. Angewandt auf Darstellungsund Gestaltungsprozesse heißt diese bewusste Haltung, dass wir erst dann, wenn wir beide Dimensionen kennen und in der Lage sind, die Differenz zwischen beiden zu begreifen, zu erleben und aktiv zu gestalten, die Vorgänge des Entwerfens und des Darstellens nicht nur als Mittel, sondern als Medien der Gestaltung ansehen, die prozesshaft und performativ sind. Diese Haltung verändert sowohl das Verständnis der Medien als generativer Anteil des Entwurfsprozesses - und in keiner Weise als Hindernis - wie 
auch die Weise, in der der Entwurfsprozess dokumentiert wird: Der Prozess wird Teil des Forschens als Suche nach einer neuen Medialität für das Entwerfen. Die sogenannten ,Arbeitszettel' stellen insofern eine essentielle entwerferische Komponente der Architektur dar. Aber auch die Architektur erweitert ihre Wirkung: Sie steht nicht nur für das Entwerfen von Gebäuden, sondern artikuliert ein Netzwerk von kulturellen und gesellschaftlichen Relationen, die durch Medien zur Sichtbarkeit gebracht werden können. ${ }^{4}$ Medien sind somit generativer Anteil des Entwerfens, das gleichzeitig als Hervorbringung und Dokumentation architektonischer Prozesse angesehen werden kann. Die Etablierung einer solchen bewussten Haltung, die der generativen Bedeutung der Medien Rechnung trägt, ist der Hauptgrund für die Einführung einer Media-AgencyTheorie.

Im vorliegenden Beitrag werden zuerst Grundfragen der Medienphilosophie mit Blick auf ihre Relevanz für die Architektur eingeführt, um dann einige Kernthemen der Medienphilosophie (Definierbarkeit des Medium-Begriffs, das Verhältnis von Wahrnehmung und Medien und die Performativität des Medialen) aufzugreifen, die eine Grundlage auch für den architektonischen Diskurs zur Medialität darstellen können. Auf diese Weise soll die anvisierte Media-Agency-Theorie klarer umrissen werden.

Medienphilosophie in der Architektur Architektur ist für die philosophische Reflexion zur Medialität paradigmatisch, weil ihr Entwurfsprozess multimedial und somit eine ständige ,mediale Grenzüberschreitung" 5 ist: Im Entwerfen werden verschiedene Medien nacheinander und nebeneinander angewandt, sodass jede Präsentation im Rahmen eines Entwurfsseminars, eines Wettbewerbs oder eines konkreten Auftrags eine Aneinanderreihung von Medien ist, die gleichzeitige Gültigkeit besitzen und letztlich ein multimediales Produkt darstellen. Diese konstante Wechselwirkung zwischen Medien macht das architektonische Projekt zu einem wahrnehmbaren Medienprisma eines architektonischen Konzeptes. Im Entwurfsprozess wird intensiv sowohl mit einzelnen Medien als auch mit der gesamten, multimedialen Artikulation experimentiert, die das Konzept auf einer medialen Projektionsfläche realisiert, in die alle Medien zusammenfließen und auf der sie sich gegenseitig ergänzen. Eine Projektidee kann durch ein Medium wie einen Plan, ein Modell oder einen Text zur Gestaltung gelangen. 
Der Entwurfsprozess ist in jedem Medium potentiell erschlossen; er kann sich in vielen Medien vollziehen oder auf spezifische mediale Räume begrenzt werden. Es ist bekannt, dass es Architekten und Architektinnen gibt, die bestimmte Medien favorisieren und bei denen eine eigene mediale Sprache zu erkennen ist - zu denken wäre etwa an die Modellarbeiten von Frei Otto, die malerischen Zeichnungen von Aldo Rossi und die diagrammatischen Zeichnungen von Zaha Hadid. Diese experimentellen Praktiken werden dann in den jeweiligen Büros zur bautechnischen Ausführung weiter verwandelt. Angesichts dieses Übersetzungsprozesses stellt sich die Frage, wie sich in dieser Übertragung, die zur Endpräsentation des Projektes führt, mediale Praktiken der jeweiligen Entwerfenden transformieren und inwieweit ihr performativ-generativer Charakter dabei erhalten bleibt oder bloß abgebildet wird, ohne die Spezifizität der jeweiligen Medien zu bewahren. Ist das nicht der Fall, handelte es sich hingegen um bloße, meist unbewusst geschehende Re-Präsentation ohne Berücksichtigung des spezifischen Potentials der involvierten Medien. Häufig lässt sich im Entwurfsund Bauprozess eine Diskrepanz zwischen einer performativen und einer rein repräsentationalen Medienverwendung feststellen: Dank performativer Medien vollzieht sich die Materialisierung eines Konzepts, das jedoch anschließend unter pragmatischen Gesichtspunkten medial wieder reduziert wird. Es kommt anders gesagt darauf an, ob die Ausführung eines Architekturkonzeptes vorwiegend auf die bloße Repräsentation und Vermittlung setzt oder ob auch die technische Ausführung in ihrem generativ-performativen Potential erfasst wird. Letzteres würde vermeiden, dass das Entwerfen eines ersten Konzeptes als kreativ-mystisches Moment - eine Art Blackbox - verstanden wird, das kaum dokumentiert wird, während die Ausführung und Darstellung des ursprünglichen Konzeptes als bloße Abbildungs- und Anpassungsvorgänge angesehen werden. Aus der Perspektive einer bewussten, performativen Haltung heraus hingegen stellen auch solche Anpassungsvorgänge einen Reflexionsraum dar, innerhalb dessen der Entwurf in der Phase der Realisierung ständig weiterentwickelt wird.

Inwieweit bestimmte Medien die Entfaltung architektonischer Bedeutung ermöglichen und wie sie zu einem neuen konzeptuellen und materiellen Verständnis von Architektur führen können, ist bisher oft vernachlässigt oder den einzelnen Kompetenzen der Entwerfenden in Büros und der Lehrenden in Entwurfsseminaren 
überlassen worden. Der Fokus auf Media Agency stellt insofern einen theoretischen und gleichzeitig praxisnahen Ansatz dar, um die agentielle, generative und performative Funktion der Medien im architektonischen Entwerfen ans Licht zu bringen und für diese einen institutionellen und wissenschaftlichen Raum in der architektonischen Praxis zu schaffen.

Dabei stellt sich zunächst die Frage, was ein Medium in der Architektur ist, da eine einheitliche Definition der Medialität bislang fehlt. So hat Stefan Trüby erst kürzlich zu Recht festgestellt: „Eine Medientheorie der Architektur steht noch aus“.6 Zwar wurde verschiedentlich historisch untersucht, wie klassische Medien - etwa die Zeichnung - sich entwickelt haben und neue Medien im digitalen Zeitalter - wie Computerprogramme - hinzugekommen sind und wie deren technisches Potential heute für die Architektur definiert werden kann. Dabei wird jedoch in der Regel nicht gefragt, was ein Medium in der Architektur ist und was mit Medialität genau gemeint ist. Meist werden mediale Praktiken in ihrer historischen und technischen Entwicklung untersucht und dabei mit Werkzeugen oder tools verwechselt, ohne eine systematische Auffassung des Medium-Begriffs anzuvisieren. Zwar gibt es einzelne Studien über die Medien der Architektur, aber bislang keine systematische Untersuchung der Medialität in der Architekturgestaltung.

Als wichtiger Beitrag zur Medienverwendung in der Architektur hat der von Wolfgang Sonne herausgegebene Band Die Medien der Architektur zu gelten, der zugleich die Forschungslücke in diesem Bereich unterstreicht. Laut Sonne ,existieren zwar mehr oder weniger gewichtige Studien zu einzelnen Medien der Architektur, der Versuch einer kritischen Übersicht über die Vielfalt der architektonischen Medien und deren signifikante Eigenschaften wurde aber bislang nicht unternommen. Noch erstaunlicher ist, dass ebenfalls in den holistisch ausgerichteten jüngeren Kulturwissenschaften und Medientheorien die spezifischen Medien der Architektur so gut wie keine Beachtung finden. Nachdem in den I970er Jahren der Versuch unternommen worden war, Architektur mit Hilfe der Sprachwissenschaften und der Semiotik als Medium zu deuten, scheint heute eine gewisse Ratlosigkeit das Thema ,Architektur und Medien' zu beherrschen“.7

Der Band von Sonne umfasst eine Sammlung unterschiedlicher Aufsätze, die einzelne Medien im Entwerfen und Wahr- 
nehmen von Architektur primär anhand von Fallstudien behandeln. Dabei werden spezifische Merkmale einiger Medien wie der Zeichnung, der Karte, der Fotografie, des Films, des Modells, des Diagramms, der Computerdarstellung und der Ekphrasis analysiert. Was im Gegenteil weiterhin aussteht, ist eine systematische Untersuchung der Medialität der Architektur, die sich nicht nur auf die prägenden Medien des Entwerfens bezieht, sondern den Entwurfsprozess selbst als mediale Synthesis begreift.

Eine solche medienphilosophische Untersuchung des Entwurfsprozesses hätte zum Ziel, zunächst eine systematische Definition des Medium-Begriffs und anschließend den spezifischen Beitrag sowie die Grenzen jedes einzelnen Mediums herauszuarbeiten. Dabei ist auch zu klären, inwieweit jedes Medium spezifische gestalterische Möglichkeiten aufschließt - und andere ausschließt. Zugleich ist die Dominanz oder die Ausblendung bestimmter Medien zu hinterfragen, wie auch Sonne betont: „Was wiederum lässt sich aus der Absenz mancher Medien schließen? Warum etwa werden die akustischen, haptischen oder olfaktorischen Seiten der gebauten Umwelt selten adäquat repräsentiert? Sind sie unwesentlich oder unbeherrschbar, oder liegt hier ein gestalterisches Entwicklungspotential der Architektur?“.8 Allgemein gesprochen machen Medien Architektur wahrnehmbar durch ihre Verbindung mit den Sinnen. Medien dürfen folglich nicht von ihrer Versinnlichungsfunktion entkoppelt werden, die in diesem Fall darin besteht, architektonische Bedeutung wahrnehmbar zu machen. Denn jedes Medium entspricht einer spezifischen sinnlichen Dimension, wie sich etwa am Fall akustischer Medien aufzeigen lässt: Durch die mediale Schulung unserer akustischen Wahrnehmung können wir die Architektur als etwas verstehen, das nicht allein und auch nicht primär visuell funktioniert. Um sich dessen bewusst zu werden, reicht es allerdings nicht aus, der akustischen Wahrnehmung eine gesteigerte Aufmerksamkeit zuteilwerden zu lassen. Vielmehr bedarf es der Erfindung und Ausübung spezifischer medialer Praktiken, die die Architektur akustisch erschließen: Das Hören geschieht nicht einfach unmittelbar, sondern benötigt Praktiken, die es wahrnehmbar und vermittelbar machen. Das heißt auch, dass eine systematische Untersuchung der Verbindung zwischen Medien und Wahrnehmung zwangsläufig zur Kritik an der gegenwärtigen Verwendung von Medien im architektonischen Entwerfen führt. So ist zum Beispiel die Dominanz visueller Medien im Unterschied zu 
weiteren, marginalisierten Medien wie den akustischen zu hinterfragen.

Die skizzierte Wissens- und Forschungslücke hinsichtlich der Medialität hängt auch damit zusammen, dass die Architektur, obwohl sie eine genuin mediale Disziplin ist, noch keine eigene Medienreflexion hat und sich nicht selten eklektisch auf verschiedene Ansätze der Medientheorie und -philosophie stützt, die wiederum die Architektur noch nicht als privilegierten Gegenstand für sich entdeckt haben. Dabei stellt sich auch die Frage, ob das Ziel einer teils versuchten, transdisziplinären Verbindung bloß darin besteht, medientheoretische Definitionen auf die Architektur anzuwenden, oder es nicht vielmehr der Entwicklung einer eigenständigen Reflexion der Medialität in der Architektur bedarf, die die spezifischen Anforderungen architektonischer Medialität kritisch hinterfragt. In diesem Beitrag wird der zweite Weg eingeschlagen, indem mit dem Begriff Media Agency eine Medienphilosophie des architektonischen Entwerfens angestrebt wird, die vom Entwurf ausgeht. Dabei werden verschiedene philosophische Ansätze auf die Architektur und genauer auf das architektonische Entwerfen nicht nur angewandt, sondern diesbezüglich reflektiert und weiterentwickelt. Das architektonische Entwerfen fungiert dabei also nicht nur als ein Anwendungsfeld der philosophischen Reflexion unter anderen, sondern stellt ein genuin architektonisches Forschungsfeld dar, aus dessen Bearbeitung sich auch Fragen ergeben, die wiederum die medienphilosophische Debatte befruchten können.

Kernthemen der Medienphilosophie Der Mangel an einer verbindlichen Definition des Medium-Begriffs betrifft nicht nur die Architektur, sondern auch die Medienphilosophie selbst. An dieser Stelle soll es jedoch vorwiegend um ein Verständnis des Begriffs gehen, insofern er für die Architektur von Relevanz ist. Wie im Folgenden außerdem deutlicher werden wird, basiert der MediaAgency-Ansatz in theoretischer Hinsicht vor allem auf der Untersuchung der Performativität des Medialen sowie des Verhältnisses von Wahrnehmung und Medien.

Eine systematische Reflexion des Medialen steht im Zentrum der Medienphilosophie, die sich insofern von der Medienwissenschaft und Medientheorie unterscheidet, wie auch Gerhard Schweppenhäuser hervorhebt: „Während die Medienwissenschaft 
an Empirie und empirischen Forschungsmethoden orientiert ist und Medientheorien sich an Bezugswissenschaften wie Literaturund Kulturwissenschaft, Soziologie, Philosophie und Psychologie sowie deren Paradigmen abarbeiten, ist Medienphilosophie die begrifflich-kategoriale Klärung der Grundlagen dessen, was unter einem „Medium“ zu verstehen ist“ . ${ }^{9}$ Während der empirische Zugang Medien in ihren konkreten historischen und kulturellen Ausführungen beschreibt, bezieht sich der philosophische Zugang auf die Bedingungen der Möglichkeit der medialen Ausführung, in dem Versuch zu zeigen, ,was Medien als Mittel des Weltzugangs und der Welterschließung leisten“. ${ }^{\text {Io }}$ Die Medienphilosophie fragt daher nach einer grundlegenden Ebene des Medialen, die sich nicht deshalb von der Ebene bestimmter Praktiken unterscheidet, weil sie theoretisch verfasst ist.

Allerdings leidet die Medienphilosophie daran, dass der Begriff des Mediums so generisch geworden ist, dass alles zum Medium werden kann, wie Dieter Mersch betont: „Der Begriff scheint ubiquitär und anwendbar auf jeden Stein, aus dem ein Haus errichtet wird, auf jede Linie, die zur Markierung eines Grenzverlaufs dient, oder auf alles Schreibwerkzeug [...], ebenso wie auf Kleidungen, die zeigen und verbergen, oder auf Pflanzen und Lebewesen [...]“. II Medien - so die von Stefan Münker verwendeten Ausdrücke - sind ,en vogue“ und „omnipräsent“, ${ }^{\text {I2 }}$ weshalb der Medienbegriff, wie Lambert Wiesing bemerkt, mehr Extension als Intension gewonnen hat: ,Man hat Medientheorien von Dingen, die ohne diese Theorie keine Medien wären: wie Energie, Wahrnehmung oder den Leib. In jedem der drei Fälle verliert der Medienbegriff beachtlich an Intension, und seine Extension nimmt bedenklich $\mathrm{zu}^{\text {“ }}{ }^{13}$ Gerade wegen seiner Ausweitung ist der Begriff des Mediums eigentümlich unterbestimmt geblieben.

Angesichts seiner omnipräsenten Verwendung stellt seine theoretische Bestimmung ein vorrangiges „Desiderat"I4 dar. Diese Bestimmung hätte zum Ziel, seine ,differentia specifica“" 15 anderen Begriffen gegenüber zu definieren. Weil der Medium-Begriff an Intension verloren und an Extension gewonnen hat, wird die Frage nach seinen spezifischen begrifflichen Merkmalen allmählich auf eine deskriptive Analyse einzelner Medien reduziert: So wird meist nicht untersucht, ob und wie ein Schreibwerkzeug ein Medium ist, sondern wie es sich im Gebrauch transformiert hat. Und dieser deskriptive, medienhistorische Zugang setzt eigentlich schon 
voraus, geklärt zu haben, was ein Medium überhaupt ist. Dabei ist fraglich, ob die Mediengeschichte die philosophische Frage ,Was ist ein Medium?' weiterhin ausblenden und ihren Medien-Begriff stattdessen schlicht auf Kommunikationsmittel in ihrer technischen Ausführung und Implementierung zurückführen kann.

Genau dieses Verständnis von Medien aber ist weiterhin dominant: Medien werden mit Apparaten und Dispositiven gleichgesetzt, ohne zu hinterfragen, ob mit dem Medien-Begriff natürliche oder künstliche Medien erfasst werden. ${ }^{16}$ Sind unsere Sinne also natürliche Medien, oder sind nur technische Geräte als Medien zu bezeichnen? Und wenn Medien mit Techniken identifiziert werden, dann wird dadurch - wie Mersch anmerkt - ,,auch der Begriff des Technischen totalisiert". ${ }^{17}$

Die allgemeine Fähigkeit von Medien, semantische Relationen wahrnehmbar zu machen und gleichzeitig die Präsenz eines Gegenstandes aufzuheben, führt zu der Frage, inwieweit zwischen natürlichen und künstlichen Medien unterschieden werden muss und die technische Implementierung der Medien wie etwa digitaler Medien eventuell eine im Vergleich zu derjenigen natürlicher Medien andere Art von Medialität darstellt. Insbesondere Hartmut Böhme und Peter Matussek haben von einer „Naturmedialität“ gesprochen und Sinne als natürliche Medien definiert: „Immer dann, wenn sinnliche Ereignisse für den Wahrnehmenden über die bloße Referenz eines Objekts des mundus sensibilis hinaus den Charakter des Performativen erhalten, kann von den Sinnen als ästhetischen Medien die Rede sein“. ${ }^{18}$

Trotz solcher Einwände werden Medien weiterhin häufig unreflektiert mit spezifisch technischen Medien oder Massenmedien assoziiert, wobei übersehen wird, dass das Mediale philosophisch gesehen gerade nicht als bloßes Dazwischen $\mathrm{zu}$ verstehen ist, ${ }^{19}$ sondern als Bedingung der Möglichkeit von Vermittlung. Genau genommen ist selbst McLuhans Definition noch nicht radikal genug: Das Medium ist nicht nur die Botschaft selbst, ${ }^{20}$ sondern deren Konstitution. Dieses Verständnis des Mediums betont schon in der platonischen Definition des ,metaxy die Vermittlungsfähigkeit und nicht deren technische Ausführung. ${ }^{21}$ Das Medium ist dabei ein Drittes, das in der Vermittlung zuvor nicht gegebene Bestandteile - wie Idee und Materie - verbindet, indem es sie im Prozess konstituiert und zur Sichtbarkeit bringt. In diesem Sinne sind Medien für jedes Weltverhältnis unabdingbar und somit 
„grundsätzlich generativ“, wie Lorenz Engell betont: „Medien sind Ermöglichungen. Dabei öffnet ein Medium einen je spezifischen Horizont der Möglichkeiten“. 22

Trotz ihrer Kritik des Medienapriorismus, deren Behandlung den Rahmen dieses Aufsatzes überschreiten würde, definiert Sybille Krämer die Funktion von Medien als ein „Wahrnehmbarmachen“. ${ }^{23}$ Medien entsprechen nicht der Wahrnehmung, geben ihr jedoch eine Form und haben eine originär performative Funktion, die darin besteht, die sinnliche Form der Wahrnehmung zu vermitteln und begreifbar zu machen. Der Gehalt der Wahrnehmung wird somit durch das Mediale vermittelt und transformiert, wie auch Wiesing bemerkt: „Hätte der Mensch keine Medien, er könnte nur sehen, was anwesend ist, er könnte nur sehen, was er auch hören, riechen und tasten kann. Nur weil es Medien gibt, ist der Mensch in der Lage, zu zwei Zeitpunkten dasselbe sehen, hören und denken zu können“. ${ }^{24}$

Insofern Medialität nicht ohne den Gebrauch zu denken ist, der in unterschiedlichen Praktiken Bedeutung sinnlich artikuliert, kann Medialität als Dimension des Akts der Aisthetisierung, der Versinnlichung in Verbindung zur Performativität gebracht werden. ${ }^{25}$ Inwieweit Medialität und Performativität systematisch verbunden sind, sollte nicht nur anhand der Sprache und des Theaters - beides privilegierte Beispiele der Performativitätstheorie sondern anhand der Gestaltungskraft von Medien im Allgemeinen untersucht werden. Das bedeutet, dass Performativität nicht nur als ein Akt der Aisthetisierung, der sich in der Rezeption vollzieht, sondern als ein Akt der Versinnlichung verstanden werden sollte, der in den Medien bereits enthalten ist. ${ }^{26}$ Das mediale Handeln wird dadurch um die Dimension der Sinnlichkeit bereichert, was in Bezug auf die Medien der Künste und der Architektur, wie bereits erwähnt, meist anhand von Fallstudien untersucht wird. ${ }^{27}$ Auf den Performativitätsbegriff als zentraler Aspekt der Medienphilosophie werde ich unten im Abschnitt zur Media Agency zurückkommen.

\section{Was ist ein Medium? Die Unterscheidung zwischen sinnlicher}

Form und Praktik Um also zu verstehen, was Medien sind, muss zuerst betont werden, dass es sich bei ihnen nicht bloß um passive Werkzeuge handelt, sondern genauer um Akte der Versinnlichung. Damit ist zweierlei gemeint: Erstens vollzieht sich schon unsere sinnliche Wahrnehmung auf eine Weise, die nicht ausschließlich 
passiv ist. Vielmehr finden in ihr fortlaufend gestalterische Prozesse statt, indem äußere Sinneseindrücke aktiv auf grundlegende Formen, auf sinnliche Schemata bezogen werden. Nur wenn diese aktivische Rolle unserer Wahrnehmung, die ich mit dem Begriff der Versinnlichung zu beschreiben versuche, berücksichtigt wird, lässt sich das kreative Potential von Medien hinreichend verstehen, die dann eben nicht mehr als bloß passive Mittel erscheinen, sondern allgemeiner als sinnliche Formen unserer Wahrnehmung, die eben diese Wahrnehmung entscheidend prägen, aber sich zugleich auch von uns prägen lassen. Zweitens aber umfasst der hier vorgeschlagene Begriff von Medien spezifische Gebrauchsweisen oder Praktiken, durch die das konstruktive Potential sinnlicher Formen durch Techniken ausgeschöpft wird.

Medien stehen somit gleichzeitig für die sinnlichen Formen unserer Wahrnehmung und deren Gebrauchsweisen. Verfestigt und eingeübt können solche Gebrauchsweisen Teil einer eigentümlichen, performativen Haltung werden.

Bezeichnen wir zum Beispiel ein Diagramm als Medium, so wird damit sowohl seine sinnliche Konstitution als hybride Form auf der Grenze zwischen Bildhaftem und Textuellem als auch deren spezifische Verwendung als analoge oder digitale Zeichnung angesprochen. Dies sind zwei zu unterscheidende Ebenen. Wenn das Diagramm etwa im architektonischen Entwerfen parametrisch erzeugt wird, handelt es sich um eine technische Verwendung, die als mediale Praktik definiert werden kann. Das Diagramm ist ein Medium nicht nur in seiner technischen Ausführung (etwa in einem Plan oder in einem Modell), sondern als sinnliche Form des Raumes - im Fall der Zeichnung die zweidimensionale Fläche, die visuell und in einigen Fällen haptisch wahrgenommen werden kann. Das Bild kann sich wiederum bis zur dreidimensionalen Fläche ausdehnen, die in der physischen Ausführung der sinnlichen Form des Modells entspricht.

Die konkreten medialen Praktiken in ihrer technischen und historischen Entwicklung bleiben von der Ebene der sinnlichen Formen nicht ausgeschlossen; sie sind im Gegenteil ihre empirische Gestaltung. Ihre konkrete Ausführung schafft die Verbindung zwischen den sinnlichen Formen und den Praktiken und sorgt für die Entwicklung von Praktiken, welche die konkrete Realisierung des Medialen sind. Mediale Praktiken zeigen, wie der Entfaltungsraum sinnlicher Formen bis an die Grenzen des Medialen gebracht 
werden kann. Dabei spielen künstlerische Praktiken als Experimentierfelder eine grundlegende Rolle. Sie experimentieren mit der sinnlichen Konstitution des Medialen, indem sie dessen Grenzen und Potentiale aufzeigen.

Gerade künstlerische Praktiken zeigen, dass die technische Ausführung vom jeweils spezifischen Gebrauch sinnlicher Formen abhängt. Bei ihnen handelt es sich insofern in exemplarischer Weise um Praktiken der Versinnlichung. Denn dieser Gebrauch ist keine bloße Umsetzung des Sinnlichen, sondern dessen Realisierung. Wie schon Gilles Deleuze deutlich macht, stellen die Werke dreier Künstler wie Pollock, Bacon und Mondrian drei verschiedene Realisierungsräume derselben sinnlichen Form ,Bild' dar und sind essentiell für die Materialisierung des Mediums. Sie erforschen die sinnliche Dimension des Bildes und bringen sie in verschiedenen Ausführungen an die Grenzen seiner Wahrnehmbarkeit, indem zum Beispiel Pollock in der haptischen Malerei die visuelle Codierung hinsichtlich der Zeichen ausblendet, etwas, das bei Rothko mit einer radikal visuellen Malerei geschieht. Das Figurative wird dabei in das Malerische verwandelt. Im Unterschied dazu realisiert Mondrian in seiner abstrakten Malerei eine andere Materialisierung des Bildes als sinnliche Form. In diesem Sinne kann das Medium ,Bild' nur als sinnliche Gestalt abstrahiert werden, sein Wesen zeigt sich jedoch erst in der Praktik, die die sinnliche Konstitution des Medialen realisiert.

An dieser Stelle ist ein Verweis auf die Theorie der negativen Medialität von Mersch angebracht, der zufolge die Definition des Medium-Begriffs „unsäglich“ bleibt, weil das Medium, oder besser gesagt: das Mediale sich der diskursiven Definition entzieht und sich in den Praktiken zeigt: „Das Mediale ist nicht, vielmehr ereignet es sich. Es handelt sich nicht um ein Sein, sondern um ein Werden“. Das heißt, seine Exponierung als Praxis ist „mit der Art und Weise ihres Vollzugs und ihrer Ausführung kurzzuschließen - mit einem Wort: mit ihrer Performativität"“. ${ }^{28}$ Die Praktiken sind somit performativ, weil sie das Medium realisieren, das nun untrennbar mit seiner materiellen Konstitution verbunden ist. Die aus den Praktiken resultierenden Artefakte sind soziale und kulturelle Konstrukte als spezifische Arten der Verkörperung. ${ }^{29}$

Es ist wichtig zu betonen, dass die skizzierte Unterscheidung zwischen Medium und Praktik heuristischer Natur ist: Sie ist eine methodische Unterscheidung, die diese Bestandteile trennt, um ihre 
jeweiligen Funktionen offenzulegen. In Wirklichkeit sind diese zwei Dimensionen eng verbunden und als mediale Praktik wahrzunehmen.

Übertragen auf das Feld der Architektur impliziert die Annahme solcher medialer Praktiken ein gesteigertes Bewusstsein für die Frage, was die Medien (Bild, Modell, Text usw.) für die sinnliche Konstitution und Wahrnehmung der Architektur bedeuten. Die Entwicklung und Kultivierung von Praktiken, die neue Bedeutungen generieren und wahrnehmbar machen, trägt letztlich auch zu einer präziseren theoretischen Analyse der Grenzen und Potentiale dieser sinnlichen Konstitution bei, womit der wissensgenerierende Anteil künstlerischer und entwurfsbasierter Praktiken angesprochen ist.

Media Agency Media Agency heißt, Medien in ihrem agentiellen Charakter zu begreifen. Angesichts der oben eingeführten Unterscheidung zwischen Medium und Praktik sind nun zwei Ebenen der Agency zu definieren: Die erste Ebene betrifft die spezifischen Eigenschaften, die ein Medium als sinnliche Form hat; dabei sind nicht technische Medien, sondern die oben genannten natürlichen Medien gemeint; die zweite Ebene betrifft die Praktik, das heißt die konkrete Realisierung und Anwendung der jeweiligen sinnlichen Form - in unserem Fall im Entwurfsprozess. Während die erste Ebene (sinnliche Form) das Gestaltungsvermögen unserer Sinnlichkeit betrifft, kann die zweite Ebene (Praktik) nicht von der Haltung absehen, die wir den Medien gegenüber einnehmen. Die oben eingeführte Performativität der Praktiken kann somit nicht von der Haltung weggedacht werden, die im konkreten Gebrauch im Fall der Architektur im Entwerfen - eingenommen wird.

In dieser Hinsicht lässt sich der agentielle Charakter des Mediums nicht vor einer subjektiven Haltung trennen. Die subjektive Haltung hat ihren Kern aber nicht abstrakt in den Intentionen des Subjekts, sondern in seiner Fähigkeit, das performative Potential des Mediums zu erkennen und zu realisieren. Die Agency des Subjekts gründet - im Kantischen Sinne - im Vermögen des Imaginierens und Versinnlichens. Aber nicht das Subjekt als solches, sondern sein tradierter Gebrauch von Medien ist zentral. Im Sinne Kittlers stellen auch neue Medien eine ständige Transformation der subjektiven Wahrnehmung dar und sind daher keine „Prothesen“ eines gleichbleibenden Subjekts. ${ }^{30}$ 
Die zwei Ebenen (Medium und Praktiken) involvieren beide Performativität. Medien sind in ihrer Gestaltungskraft an sich performativ: Mit einem Bild können wir bestimmte Aspekte der Realität wahrnehmen, die sich im Medium Text anders darstellen würden. Medien prägen auf diese Weise unseren sinnlichen Zugang zur Bedeutung, oder anders gesagt: die Bedeutung wird durch Medien mitdefiniert. Wenn wir etwas ausdrücken, geht es nicht nur um den Inhalt, das Was unserer Kommunikation, sondern auch um die Form, das Wie unseres Ausdrucks. Dabei entsteht die Versinnlichung jeder Bedeutung, die nicht von ihrem Inhalt zu trennen ist. ${ }^{3 \mathrm{I}}$ Übertragen auf die Architektur würde das heißen, dass ein Entwurfsprozess, im dem primär mit bildgebenden Verfahren gearbeitet wird, die architektonische Bedeutung durch eine bildhafte Form unserer Wahrnehmung prägt. Nimmt diese bildliche Prägung überhand, so werden andere sinnliche Aspekte der architektonischen Wahrnehmung und des Entwerfens ausgeblendet, denn Architektur ist - wie schon Böhme und Pallasmaa pointiert bemerkt haben keine rein visuelle Kunst.

Diese Ebene der Medialität als sinnliche Gestaltung betrifft nicht den Gebrauch an sich, sondern, wie schon erwähnt, die Haltung, die wir in der Verwendung von Medien einnehmen. Dabei können die zwei bereits zu Beginn eingeführten Haltungen unterschieden werden: die instrumentelle und die performative. Ihre Differenz ist nun auf der Grundlage der Performativitätstheorie weiter zu spezifizieren. ${ }^{32}$

Austin hebt mit dem Performativen eine andere Ebene der Sprache hervor, die nicht nur in einer referentiellen Ausprägung, durch die wir Bezug auf Gegenstände nehmen, sondern in der performativen Realisierung besteht, durch die wir Handlungen und Fakten kraft der Sprache realisieren. ${ }^{33}$ Die Sprache kann somit nicht von ihrer affordance getrennt werden: Ihre Wirkungskraft hängt somit von ihrem Gebrauch ab, der auch einen konventionellen Charakter hat. Erika Fischer-Lichte beschreibt eine ähnliche Unterscheidung im Theater: ,Während die referentielle Funktion auf die Darstellung von Figuren, Handlungen, Beziehungen, Situationen etc. bezogen ist, richtet sich die performative auf den Vollzug von Handlungen - durch die Akteure und zum Teil auch durch die Zuschauer - sowie auf ihre unmittelbare Wirkung“.34 Fischer-Lichte liest die europäische Geschichte des Theaters als 
„Geschichte von Umstrukturierungen und Neubestimmungen des Verhältnisses zwischen beiden Funktionen“. ${ }^{35}$

Karen Barad wiederum überträgt die Begrifflichkeit performativer Praktiken auf das Feld der Physik und der Gender Studies: „Im Unterschied zum Repräsentationalismus, der uns über oder außerhalb der Welt ansiedelt, auf die wir angeblich nur reflektieren, hebt ein performativer Ansatz das Verständnis des Denkens, Beobachtens und der Theoriebildung als Praktiken der Auseinandersetzung mit der Welt, in der wir existieren, und als Teil dieser Welt hervor"..$^{6}$

Dieses performative Verständnis verändert vor allem die Auffassung der Praktiken, die keine bloßen Beobachtungsinstrumente oder Geräte sind, die ,als neutrale Sonden der natürlichen Welt eingesetzt werden können“,37 sondern ,grenzziehende Praktiken" $3^{8}$ die die Welt immer wieder materiell rekonfigurieren: „Apparate sind die Bedingungen der Möglichkeit für bestimmte Grenzen und Eigenschaften von Objekten und Bedeutungen verkörperter Begriffe innerhalb des Phänomens"“. 39 In diesem Sinne unterscheidet auch Barad zwischen Apparaten, die als reine Werkzeuge verstanden werden, und Medien als Praktiken, die im konkreten, historisch gegebenen Gebrauch Bedeutungen oder - in der starken Variante - sogar Objekte materialisieren. Nach Barad lässt sich also die instrumentelle Haltung nicht von einem problematischen Repräsentationalismus trennen, der auf vorgegebene Vorstellungen fokussiert und Praktiken als reine Werkzeuge ansieht. Die instrumentelle Haltung kann somit als rein repräsentationalistisch definiert werden, weil durch sie Bedeutung bloß re-präsentiert und nicht generiert wird. Im architektonischen Entwerfen steht diese Haltung für eine Auffassung, die den Vorstellungen der Entwerfenden eine größere Bedeutung beimisst als dem generativen Anteil der Medien.

Die Fähigkeit des Experimentierens Die Kritik am Repräsentationalismus eröffnet eine erkenntnistheoretische Ebene, die das Performative von einer spezifischen Ästhetik entkoppelt. Performativ sind somit nicht nur Artefakte und Praktiken, die jede Form der Repräsentation überwinden und auslöschen - wie es in vielen architektonischen Installationen und Theater-Performances beim Experimentieren an den Grenzen zur Repräsentation geschieht sondern auch die grundlegende Haltung, die wir im Experimen- 
tieren mit medialen Praktiken einnehmen. In der Architektur dient dieses Experimentieren der Erforschung von Potentialen jedes Mediums als einer sinnlichen Form unserer Erfahrung, die durch Praktiken untersucht und immer neu entdeckt wird. Dies ermöglicht, dass Konzepte nicht einfach immer wieder durch Medien repräsentiert werden, die als bloße Werkzeuge dienen. Stattdessen generieren Medien in ihrer Agency inhaltliche Bedeutung und damit wiederum architektonische Konzepte.

Auch wenn Architekten und Architektinnen zuweilen den Eindruck haben, auch ohne externe Dispositive in ihren geistigen Prozessen den Raum zu gestalten, hat auch diese geistige Imagination eine mediale Dimension, da wir schon in unserem Geist zeichnen, schreiben und modellieren. Diese Dimensionen findet dann früher oder später in den externalisierten, technischen Dispositiven eine Entsprechung und kann sich zu medialen Praktiken weiterentwickeln. Ob innerlich oder äußerlich vollzogen: Das architektonische Entwerfen kann - wie das Denken im Allgemeinen - nicht vom Medialen absehen, wie schon Kant bemerkt: „Ich kann mir keine Linie, so klein sie auch sei, vorstellen, ohne sie in Gedanken zu ziehen, d. i. von einem Punkte alle Teile nach und nach zu erzeugen, und dadurch allererst diese Anschauung zu verzeichnen".40

Konzepte verändern sich im Prozess der Gestaltung, und das Experimentieren mit medialen Praktiken erschließt neue Räume. Das Experimentieren, das Eintauchen in die Welt der Medialität bildet die Voraussetzung, um zu verstehen, was Media Agency bedeutet: Ohne diese für das Prozesshafte des Entwerfens offene Haltung bleiben mediale Praktiken bloße Instrumente, die wir allein als neutrale Sonden oder als lästige Hindernisse unseren Konzepten gegenüber verstehen. Diese Haltung betrifft alle sinnlichen Dimensionen, mit denen mediale Praktiken verbunden sind: Es geht bei ihr um die textuelle, diskursive Dimension - eine Sprache, die unsere Gedanken nicht nur begleitet, sondern in Form bringt und dadurch konstituiert; sie betrifft die Materialität, die im Modell nicht nur verwendet, sondern generiert wird; sie beeinflusst alle analogen und digitalen Dispositive, die die Zeichnung nicht nur implementieren, sondern immer neu erfinden. Kurz, sie berührt alle Dimensionen der Sinnlichkeit, die durch mediale Praktiken realisiert werden, Raum wahrnehmbar machen und Architektur verkörpern. 
Diese performative Haltung den Medien gegenüber kann auch als bewusste Haltung definiert werden. Dabei sollte jedoch ein Missverständnis vermieden werden, das die Idee eines bewussten, kreativen Prozesses mit sich bringen kann. Denn dass dieser Prozess bewusst ist, heißt nicht, dass er vollkommen kontrollierbar ist. Sein bewusster Anteil betrifft vielmehr die Vorbereitung, die Ausbildung bestimmter Fähigkeiten der Entwerfenden - mehr als ihre konkreten Handlungen. Es geht anders gesagt um die Werkzeugkiste, die die Entwerfenden mit sich tragen und im Entwurfsprozess meist implizit anwenden und weiterentwickeln. Der Entwurfsprozess enthält zwangsläufig eine unkontrollierbare Komponente, die generativ ist. Aber der Handlungsraum enthält gewisse Grenzen, innerhalb derer die Entwerfenden agieren und experimentieren. Es sind Grenzen, mit denen unterschiedlich umgegangen werden kann: Ohne Vorwissen lässt sich zwar ,blind' experimentieren, wobei Widerstände gespürt und Grenzen als unüberwindbare Hindernisse wahrgenommen werden. Aus einer bewussten Haltung heraus aber lassen sich Grenzen theoretisch und praktisch erforschen, um anschließend im konkreten Gebrauch, in der Erfahrung, aktiv mit ihrem Widerstand zu arbeiten. Der Handlungsraum ist in diesem zweiten Fall ein Möglichkeitsraum, in dem Prozesse generiert werden. Und diese Generierung betrifft vor allem die Medien als sinnliche Formen, die sich bereits im Architekturstudium theoretisch und praktisch erforschen lassen, um den Gestaltungsprozess nicht nur als isolierten, kreativen Raum, sondern in seinem generativen Potential zu begreifen, in dem neue Praktiken erfunden werden können, um so die spezifische Medialität der Architektur aufzuzeigen und gleichzeitig zu transformieren - wie auch den Raum, der mit allen unseren Sinnen gebaut und gelebt wird. Dieses Element des hier vertretenen Medialitätsverständnisses kann daher auch als ,propädeutisch' bezeichnet werden, weil es ins Spiel kommt, bevor die Entwerfenden konkrete Entwurfserfahrungen machen; es geht dabei um ein Grundgerüst an Fähigkeiten, die ausgebildet werden, um den Raum in seiner sinnlichen Fülle kennenzulernen. Diese Vorbereitung betrifft einerseits die Theorie, als Lehre des Raumes, und andererseits die Praxis, als Experimentieren mit und im Raum.

Dabei gilt es vor allem einen zentralen Aspekt der Medialität zu untersuchen, und zwar die Konnotation der Medien wahlweise als opak oder als transparent. Im Entwurfsprozess können Medien 
dann als opak definiert werden, wenn sie Widerstand leisten und in den jeweiligen Praktiken präsent sind; transparent sind sie hingegen, wenn sie widerstandslos verschwinden und sich nicht mehr zeigen. ${ }^{4}$ I Letzteres kann grundsätzlich in der Verwendung aller Medien des architektonischen Entwerfens geschehen; besonders gut lässt es sich jedoch an der Arbeit mit digital generator design tools als Beispiel veranschaulichen. Wenn die Entwerfenden sie quasi automatisch bedienen und durch die ständige Wiederholung die Grenze zur Maschine nicht mehr spüren, erleben sie die Transparenz dieser Medien. Wenn sie jedoch versuchen, mit diesen Programmen $\mathrm{zu}$ experimentieren, sie in der Programmierung so zu beherrschen, dass sie mit ihrer Hilfe auch andere Gestaltungund Darstellungsprozesse entwickeln können, dann arbeiten sie im bewussten Umgang mit den Medien und erleben somit ihren Widerstand. Das Entwerfen ist so ein ständiges Wechselspiel zwischen Transparenz und Opazität der Medien. Entwurfspraktiken auszuführen, erfordert also eine bewusste Haltung, da die Entwerfenden in ihrer Medienverwendung einer Strategie folgen. Das heißt wiederum nicht, dass der Prozess vollständig kontrollierbar ist und die Medien nur in ihrer Opazität präsent sind. Im Gegenteil bereiten Entwerfende das Verschwinden der Medialität auch bewusst vor, und das geschieht implizit. Eine Praktik, die dabei oft angewandt wird, ist die experimentelle Wiederholung, die die Medien zum Verschwinden bringt, wie die Überlegungen und die Praxis der Wiederholung von vielen Entwerfenden - nicht zuletzt von Aldo Rossi - zeigen. ${ }^{42}$ Auf der anderen Seite betrifft die Opakisierung der Medien, d.h. der Moment, in dem Medien sich in ihrer Präsenz zeigen, auch ihre technische Komponente. Im architektonischen Entwerfen zeigt sich dieser Aspekt in der Verwendung aller analogen und digitalen Medien, die - vom Bleistift bis hin zu digitalen Programmen - als mediale Praktiken auch technische Apparate sind. Doch vor allem am Beispiel digitaler Programme lässt sich die Relevanz des opaken Moments der Medialität veranschaulichen. Denn im architektonischen Entwerfen sind CAD- und BIMAnwendungen für die Entwerfenden vorprogrammiert und bieten daher hinsichtlich der architektonischen Darstellung begrenzte Möglichkeiten, die die Entwerfenden selbst selten steuern können. Die Opazität zeigt sich in diesem Fall als ein Hindernis, das in der repräsentationalen (instrumentellen) Haltung zur passiven Annahme des technischen Apparats führen kann. Eine performa- 
tive Haltung dagegen kann zum Medienwechsel oder im Idealfall zur Intervention der Entwerfenden auf der Ebene der Programmierung führen. In diesem Sinne sind die Entwerfenden aus der Sicht des Media-Agency-Ansatzes nicht nur Nutzer, sondern Hacker der Medialität: Sie sind nicht nur in der Lage, technische Apparate hinsichtlich ihrer medialen Qualität zu verwenden, sondern auch dazu, sie zu transformieren und zu modellieren. Auf diese Weise entwickeln sie die architektonische Gestaltung generativ, anstatt sie lediglich technisch zu implementieren. Dabei enthält der Media-Agency-Ansatz sowohl eine ästhetische als auch eine ethische Komponente: Die ästhetische liegt in der Relation zwischen Wahrnehmung und Gestaltung unseres Lebensraums; aus ethischer Sicht tritt die ,Freiheit' der Entwerfenden in den kritischen Fokus - beispielsweise hinsichtlich der wenig erforschten Verstrickungen zwischen Programmierung, Verbreitung und Vermarktung von Entwurfsprogrammen, die die Gestaltung unseres Lebensraums vorprägen. Auch experimentelle Praktiken, die das architektonische Entwerfen auf den ersten Blick in die Nähe der Kunst rücken und es in gewissem Maße entfunktionalisieren, berühren den ethischen Kern einer Architektur, die sich ihrer eigenen medialen Praktiken bewusst werden möchte und deshalb nach neuen medialen Erschließungen sucht.

Die experimentelle, immanente und performative Haltung betrifft schließlich sowohl das Hervorbringen als auch das Wahrnehmen von Raum. Das hervorgebrachte Werk - zugleich Umformung unseres Lebensraums - ist wiederum Gegenstand der Wahrnehmung, der gegenüber ebenfalls eine Haltung eingenommen wird. Wir können den Gegenstand in seiner äußerlichen Natur begreifen und ihn durch klassifizierende und bewertende Gedanken sofort repräsentational etikettieren oder direkt kopieren. Doch wir können mit diesem neuen Gegenstand ebenso experimentieren und ihn mithilfe verschiedener medialer Praktiken erforschen. Aus diesem Grund lassen sich in der Ausbildung junger Architekten und Architektinnen das Wahrnehmen, das Betrachten, das Erfassen und das Erforschen nicht von der konkreten Gestaltung trennen: Wahrnehmen und Hervorbringen sind komplementäre Teile des Entwerfens. 


\section{Anmerkungen}

1 John Dewey, Kunst als Erfahrung, Frankfurt a. M. 1987, S. 229.

2 Ebd.

3 Ebd., S. 230.

4 Siehe dazu das von Nishat Awan, Tatjana Schneider und Jeremy Till herausgegebene Buch Spatial Agency. Other Ways of Doing Architecture (Oxon/UK 2011), dem das Verdienst zugerechnet werden kann, den Begriff der Agency im Rahmen der Architektur behandelt zu haben, die weit gefasst nicht nur Gebäude entwirft, sondern kulturelle und gesellschaftliche Prozesse artikuliert.

5 Jörg H. Gleiter, Architekturtheorie heute, Bielefeld 2008, S. 43f.: „Dies wirft ein neues Licht auf eine alte, jedoch zwischendurch vernachlässigte Tatsache, dass die Architekturpraxis sich immer diesseits und jenseits der Bilder bewegt, dass die Architektur immer eine Praxis medialer Grenzüberschreitung ist von den bildhaften Visionen in Skizze und Zeichnung zu ihrer Vergegenständlichung im Raum".

6 Siehe dazu Stephan Trüby, „Medientheorie“, Arch+, 221, 2015, S. 106-109.

7 Wolfang Sonne, „Die Medien der Architektur", in: Ders. (Hg.), Die Medien der Architektur, München u.a. 2011, S. 8-10.

8 Sonne, „Die Medien der Architektur” (Anm. 7), S. 13.

9 Gerhard Schweppenhäuser, „Einleitung”, in: Ders. (Hg.), Handbuch der Medienphilosophie, Darmstadt 2018, S. 13.

10 Ebd. S. 14. Dazu siehe auch Dieter Mersch, „Philosophien des Medialen. ,Zwischen' Materialität, Technik und Relation", in: Schweppenhäuser, Handbuch der Medienphilosophie (Anm. 9), S. 27.

11 Dieter Mersch, Aura und Ereignis. Untersuchungen zu einer Ästhetik des Performativen, Frankfurt a. M. 2002, S. 55.

12 Stefan Münker, „Beantwortung der Frage: Was ist Medienphilosophie?", in: Stefan Münker, Alexander Roesler und Mike Sandbothe (Hg.), Medienphilosophie. Beiträge zur Klärung eines Begriffs, Frankfurt a. M. 2003, S. 7. Dazu siehe auch Stefan Münker, Philosophie nach dem »Medial Turn«. Beiträge zur Theorie der Mediengesellschaft, Bielefeld 2009.

13 Lambert Wiesing, "Was sind Medien?", in: Stefan Münker und Alexander Roesler, Was ist ein Medium?, Frankfurt a. M. 2008, S. 239.

14 Münker, "Beantwortung der Frage: Was ist Medienphilosophie?" (Anm. 12), S. 7.

15 Wiesing "Was sind Medien?" (Anm. 13), S. 239. Vgl. auch Matthias Vogel, Medien der Vernunft, Frankfurt a. M. 2001, S. 13. 
16 Siehe dazu Hartmut Böhme und Peter Matussek, „Die Natur der Medien und die Medien der Natur", in: Münker und Roesler, Was ist ein Medium? (Anm. 13).

17 Mersch „Philosophien des Medialen“ (Anm. 10), S. 26.

18 Böhme und Matussek, „Die Natur der Medien und die Medien der Natur" (Anm. 16), S. 103. Dazu auch Martin Seel, "Medien der Realität und Realität der Medien", in: Sybille Krämer (Hg.), Medien, Computer, Realität, Frankfurt a. M. 1998, S. 260.

19 Alexander Roesler, "Medienphilosophie und Zeichentheorie”, in: Münker, Roesler und Sandbothe (Hg.), Medienphilosophie. (Anm. 12), S. 39.

20 Marshall McLuhan, Die magischen Kanäle - Understanding Media, Dresden 1995.

21 Siehe dazu Dieter Mersch, "Meta/Dia. Zwei unterschiedliche Zugänge zum Medialen", in: Lorenz Engell und Bernhard Siegert (Hg.), Zeitschrift für Medien- und Kulturforschung (ZMK), 2010, S. 185-208.

22 Lorenz Engell, „Tasten, Wählen, Denken. Genese und Funktion einer philosophischen Apparatur", in: Münker, Roesler und Sandbothe (Hg.), Medienphilosophie (Anm. 12), S. 54. Dazu auch Stefan Münker, "Was ist ein Medium? Ein philosophischer Beitrag zu einer medientheoretischen Debatte", in: Münker und Roesler, Was ist ein Medium? (Anm. 13), S. 331: „Während wir mit Medien nur machen können, was Medien uns zu machen erlauben, könnten wir ohne Medien nichts von dem tun, was Medien uns zu machen ermöglichen".

23 Sybille Krämer, „Erfüllen Medien eine Konstitutionsleistung? Thesen über die Rolle medientheoretischer Erwägungen beim Philosophieren", in: Münker, Roesler und Sandbothe (Hg.), Medienphilosophie (Anm. 12), S. 83: „Medien phänomenalisieren und machen also Bezugnahme möglich".

24 Lambert Wiesing, "Was sind Medien“ (Anm. 13), S. 248. Die Frage, ob dann für die Annahme einer reinen Wahrnehmung argumentiert werden kann, die für die reine Präsenz unserer leiblichen Anwesenheit steht, die erst in einem zweiten Moment von Medien realisiert und zu Schau gestellt werden kann, ist eine wichtige Frage auf der Grenze zwischen Phänomenologie und Medienphilosophie. Dazu Dieter Mersch, Aura und Ereignis (Anm. 11).

25 Dass sowohl Performativität und Medialität "Dimensionen von Akten der Aisthetisierung" sind, ist eine Hypothese, die Krämer entwickelt und die weiter verfolgt zu werden verdient, vgl. Sybille Krämer, "Was haben `Performativitätı und `Medialitätı mitein- 
ander zu tun? Plädoyer für eine in der >Aisthetisierung ' gründende Konzeption des Performativen", in: Sybille Krämer (Hg.), Performativität und Medialität, München 2004, S. 14: „Die Bedeutung von ,Performativität' ist in einer zeitgenössisch interessanten und anschließbaren Weise gar nicht ohne einen Bezug auf Medialität zu begreifen".

26 Siehe dazu Lidia Gasperoni, Versinnlichung. Kants transzendentaler Schematismus und seine Revision in der Nachfolge, Berlin 2016.

27 Ein Beispiel dafür ist der von Jutta Eming, Annette Jael Lehmann und Irmgard Maassen herausgegebene Sammelband Mediale Performanzen, Freiburg im Breisgau 2002.

28 Mersch, "Philosophien des Medialen“ (Anm. 10), S. 30.

29 Mit Praktik (practice) ist im Sinne von Dana Cuff (Architecture: The Story of Practice, MIT Press 1991, S. 4) "the embodiment, indeed the expression, of the practitioner's everyday knowledge".

30 Friedrich A. Kittler, "Geschichte der Kommunikationsmedien“, in: Jörg Huber, Alois Martin Müller (Hg.), Raum und Verfahren, Basel 1993, S. 188.

31 Siehe dazu Lidia Gasperoni, "Die Vernunft in den Sinnen", der architekt, 4/2019, S. 22-27.

32 Siehe dazu Uwe Wirth ( $\mathrm{Hg})$, Performanz. Zwischen Sprachphilosophie und Kulturwissenschaften, Suhrkamp 2002.

33 Das Buch von John L. Austin (Zur Theorie der Sprechakte, Stuttgart 1972) hat die Aufmerksamkeit hinsichtlich des Performativitätsbegriffs auf das Sprechen gerichtet und bleibt der philosophische Bezug der Performativitätstheorie, die von Judith Bluter um eine politische Dimension erweitert worden ist - siehe dazu Judith Butler, Excitable Speech. A Politics of the Performative, New York, London 1997. Zur Bedeutung der Sprechakttheorie siehe den von Uwe Wirth herausgegebenen Sammelband Performanz (Anm. 32), der eine Reihe der grundlegenden Texte enthält, die im ersten Teil auf die sprachphilosophischen und literaturwissenschaftlichen Aspekte, im zweiten Teil auf die Verwendung des PerformanzBegriffs in der Anthropologie, Theaterwissenschaft und GenderStudies konzentriert ist. Sybille Krämer, "Was tut Austin, indem er über das Performative spricht? Ein anderer Blick auf die Anfänge der Sprechakttheorie", in: Jens Kerscher, Dieter Mersch (Hg.), Performativität und Praxis, München 2003, S. 19-33. Als wissenschaftliche Untersuchungen der Performativität im Theater sind vor allem die Arbeiten von Erika Fischer-Lichte zu erwähnen: Ästhetische Erfahrung: Das Semiotische und das Performative, Tübingen 
2001, Performativität und Ereignis, Tübingen 2003 und Ästhetik des Performativen, Frankfurt a. M. 2004.

34 Erika Fischer-Lichte, "Grenzgänge und Tauschhandel. Auf dem Wege zu einer performativen Kultur", in: Wirth, Performanz (Anm. 32), S. 279.

35 Ebd.

36 Karen Barad, Agentieller Realismus, Frankfurt a. M., 2017, S. 9.

37 Ebd. S. 23.

38 Ebd. S. 21.

39 Ebd. S. 26.

40 Immanuel Kant, Kritik der reinen Vernunft, A 162f., B 203.

41 Siehe dazu Sybille Krämer, „Medien zwischen Transparenz und Opazität. Reflexionen über eine medienkritische Epistemologie im Ausgang von der Karte", in: Markus Rautzenberg und Andreas Wolfsteiner (Hg.), Hide and Seek. Das Spiel von Transparenz und Opazität, München 2010, S. 217: „Medien an-aisthetisieren sich im störungsfreien Gebrauch. Im Unterschied zu gewöhnlichen Instrumenten und Werkzeugen, im Unterschied aber auch zu Zeichenvorkommnissen, blenden Medien ihre eigene Materialität und Sinnlichkeit aus, solange sie Anderes zu vergegenwärtigen haben. Wir können dabei auch von einem Latentbleiben der Medien sprechen. Medien machen etwas wahrnehmbar, indem sie selbst dabei zurücktreten und unterhalb der Schwelle des Wahrnehmens für den Rezipienten verbleiben".

42 Siehe dazu Aldo Rossi, Wissenschaftliche Selbstbiographie, Zürich 2014. 


\section{Die Debatte über Agency in der Architekturtheorie}

,Agency“ ist seit zehn Jahren ein wiederkehrendes Thema in Architekturdebatten. Dies hat zu einer Reihe von wichtigen theoretischen Positionen geführt, welche auf Konferenzen, in spezialisierten Zeitschriften und entsprechenden Sammelbänden entwickelt und diskutiert wurde. So lassen sich zum Beispiel allein im Jahr 2009 mindestens vier Ausgaben wichtiger Architekturzeitschriften finden, die der Idee der Agency gewidmet sind: Agency in Architecture, Footprint (4); Agency and the Praxis of Activism, Field Journal 3 (I); Architectural Research Quarterly I3 (2) und Perspecta, Agency 45. ${ }^{\mathrm{I}}$ Ein Hauptbezugspunkt dieser Debatten war die Konferenz, die 2008 von der Forschungsgruppe „The Agency“ der Universität Sheffield als der fünfte Kongress der AHRA (Architectural Humanities Research Association) organisiert wurde. Die Ergebnisse dieses Treffens wurden in unterschiedlichen Artikeln der erwähnten Zeitschriften abgedeckt und diskutiert. Dieselbe Forschungsgruppe veröffentlichte zudem 20 Io die Gemeinschaftspublikation Agency. Working with Uncertain Architectures ${ }^{2}$ und 20 I I gaben Jeremy Till, Tatjana Schneider und Nishat Awan nicht nur Spatial Agency. Other Ways of Doing Architecture ${ }^{3}$ heraus, sondern sie entwickelten auch eine Online-Ressource desselben Namens, wo sie über die Untersuchungen in diesem Feld nachdenken und den zahlreichen Entwurfs- und Bauprozessen eine Stimme verleihen konnten, die gemäß dieser Vorstellungen von Agency ausgeführt wurden: www.spatialagency.net

Allerdings ist der Sinn, den man dem Begriff der Agency in den Debatten zuschreibt, alles andere als einheitlich: Schneider und Till deuten zum Beispiel an, dass sich die Vorstellung von Agency in der Architektur auf konservative Elemente beziehen kann, wie 
etwa den Fall der Architekten und Architektinnen, die ,im Auftrag“ des Bauunternehmers, des Kunden, des Entwicklers oder anderer handeln. ${ }^{4}$ Sowohl Schneider und Till als auch Ana Paula Baltazar und Silke Kapp lehnen diese Konzeption unmittelbar ab, insofern sie „Akteure impliziert, welche vollständig durch ,andere“ bestimmt und damit politisch annulliert werden". .5 Anders ausgedrückt, steht hinter der Betonung der Vorstellung von Agency, insofern sie sich gegen eine heteronome Bestimmung einer Handlung richtet, vielmehr folgende Absicht: Die im Entwurfs- und Bauprozess involvierten Handlungen sollen für die Beteiligung einer weitaus größeren Anzahl von Akteuren geöffnet werden, wobei ein besonderer Schwerpunkt auf deren politischen und sozialen Rollen liegt. Das Ziel besteht daher darin, eine Art von sozialem Wandel zu bewirken, indem deutlich gemacht wird, dass die Figur der Architektin oder des Architekten hier nur noch eine unter vielen darstellt, und indem die Partizipation all jener betont wird, die keine Berücksichtigung finden in der einfachen Vision von Architektinnen und Architekten als ,diejenigen, die Ideen haben, als Autorinnen beziehungsweise Autoren dieser Ideen agieren und Projekte zur Umsetzung dieser Ideen ausführen". 6

Auf diese Weise will der Diskurs über Agency in der Architektur einerseits die Komplexität der großen Anzahl von Handlungen und Akteuren hervorheben, die bei der Konstruktion jenseits der Vorstellungen von Architektinnen und Architekten zusammenkommen: von den Anforderungen des Kunden an das Budget, oder von den Einschränkungen, die sich aus gesetzlichen Vorgaben oder solchen der Stadtplanung ergeben, bis hin zu den unterschiedlichen Eingriffsmaßnahmen durch Kommunen oder Nachbarn, durch Denkmalschützer und Repräsentanten der Regierung oder die Stadtverwaltung. ${ }^{7}$ Hier intervenieren nicht nur zahlreiche Leute mit unterschiedlichen Interessen und Zielen, sondern auch eine beträchtliche Anzahl von Normen, Standards und Regulierungen, wie unlängst von Faulconbridge ${ }^{8}$, Street ${ }^{9}$ oder Kraft ${ }^{10}$ betont wurde. Es spielen aber auch entscheidende materielle Bedingungen eine zentrale Rolle, wie von den Autoren der AkteurNetzwerk-Theorie hervorgehoben wurde. Auf der anderen Seite weist allerdings auch eine Vielzahl der hier entwickelten AgencyTheorien einen eindeutig aktivistischen Schwerpunkt auf, der in vielen dieser Arbeiten ganz ausdrücklich anerkannt wird. Dieser Schwerpunkt steht dabei für den Versuch, Raum zu lassen für eine 
Baupraxis, in der die Nutzer, oder jene mit wenig anfänglicher Kontrolle und Einfluss auf den Prozess einen Zuwachs an Einflussmöglichkeiten beziehungsweise Macht erlangen, und ihre eigenen Formen der Agency dann mehr Aufmerksamkeit bei der Entwicklung von kollektivem Handeln erfahren.

Agency, Intention und Kontrolle In beiden Fällen wird durch die Betonung des Begriffs von Agency als Bestandteil des Entwurfsund Bauprozesses auf eine Konzeption von Handeln verzichtet, die sich lediglich auf die Intentionen der Architektin beziehungsweise des Architekten stützt, und in welcher diese durch eine hierarchische Struktur als Autorin beziehungsweise Autor sämtliche Autorität und Kontrolle über den Prozess und all diejenigen inne haben, die an ihm partizipieren. Das schlichte Modell von Architektur, in dem Architektinnen oder Architekten über eine Intention verfügen und eine Idee entwickeln, welche dann unmittelbar umgesetzt und in einem Gebäude verwirklicht wird, entspricht einer ziemlich eigentümlichen Perspektive auf die Arten von Handlung, die während des Entwurfs- und Bauprozesses stattfinden, nämlich einer Handlung, bei der Individuen alle ihre Ergebnisse unter Kontrolle haben, sei es in Gestalt materieller Produkte (die bebaute Umgebung), sei es in Gestalt der Handlungen, die während dieses Prozesses ausgeführt werden. Diese Art von Ansatz findet sich in der philosophischen Handlungstheorie in Gestalt von planungsbasierten Theorien, in denen eine Handlung verstanden wird als die Festlegung und Ausführung von Plänen, und die letztlich auf einen radikal intentionalistischen Ansatz des Handelns gegründet ist. Beth Preston bezeichnet diese Position als „Modell zentralisierter Kontrolle“, das ihres Erachtens den Kern von Handlungstheorien seit Aristoteles ausmacht. ${ }^{\text {II }}$

Für die Architektur hat dieses Handlungsmodell weitreichende Konsequenzen: Erstens wäre in diesem Kontext Kreativität oder jegliche Form schöpferischer Agency lediglich in der Bestimmung der Intention zu Anfang des Prozesses wirksam und würde damit der prozesshaften Natur des Entwerfens und des Bauens und ihren performativen Elementen nicht gerecht werden. Das Schöpferische wird hier oft verknüpft mit den Begriffen des Visionären, der Inspiration oder Innovation, die sich alle ohne jegliche zeitliche Entfaltung zu einem abstrakten Zeitpunkt einstellen, einem Zeitpunkt, der einerseits verknüpft ist mit einem anfänglichen Moment der 
Erleuchtung, anderseits aber mit den endgültigen Ergebnissen des gesamten Prozesses. ${ }^{\text {I2 }}$ Abgesehen von seinen Abweichungen vom Standard oder vom ursprünglichen Plan findet der Prozess selbst dabei niemals wirkliche Berücksichtigung. Zweitens begünstigt diese intentionalistische Auffassung des Handelns eine repräsentationalistische Konzeption von Architektur, in der das Gebäude schlicht die Repräsentation und Umsetzung der Intentionen oder Ideen der Architektin oder des Architekten darstellt, was einmal mehr ablenkt vom performativen Charakter des Entwurfs- und Bauvorgangs. Drittens schließlich verfügen sowohl die Handlungen derer, die am aktuellen Bauprozess teilnehmen, jedoch nicht in die Planung eingreifen, sowie die materiellen Bedingungen, die dieser Prozess beinhaltet, lediglich über eine instrumentelle Rolle, die der Intention der Architektin oder des Architekten untergeordnet ist, denen eine zentrale Position der Autorität und Kontrolle zukommt. Entsprechend werden Medien von dieser Warte aus als nachrangiges Mittel angesehen, das sich im Idealfall vollständig der zuvor gefassten Intention angleicht, indem es seinen eigenen Widerstand und seine Präsenz zurücknimmt, sich an die ursprüngliche Idee anpasst und diese klarer zum Ausdruck bringt. Anders ausgedrückt: Aus dieser Sicht verfügen Medien in diesem Kontext über keinerlei eigentümliche und intrinsische Agency. Darüber hinaus bestimmt jene Intention nicht nur den Verlauf des Entwurfs- und Bauprozesses und seiner Ergebnisse, sondern auch die Kriterien für deren Bewertung: Sowohl der Wert des Prozesses selbst, wie auch derjenige der Produkte wird danach bemessen, inwieweit sie der ursprünglichen Intention oder dem ursprünglichen Plan entsprechen. Aus all diesen Gründen sind architekturbezogene Agency-Theorien bestrebt, ein Alternativmodell des Handelns zu entwickeln, wo die jeweiligen unterschiedlichen Formen der Agency all jener (seien sie menschlich oder nicht) betont werden, die am Entwurfs- und Bauprozess teilhaben. Daher sollen diese Formen von Agency in der ihnen angemessenen Weise so verstanden werden, dass sie (eher) horizontal und nicht hierarchisch unter allen Beteiligten verteilt sind.

Ganz im Einklang mit der Kritik an dieser Vorstellung von der Autorität von Architektinnen und Architekten wirft Colin Lorne die Frage auf, ob das Bild, welches man sich von diesem Beruf macht, nicht durch eine Ideologie der Kontrolle geprägt ist, in der eine Gruppe, deren gemeinsame Identität durch eine bestimmte 
Ausbildung definiert wird, in einem kapitalistischen Wirtschaftssystem ,den Versuch unternimmt, ihre Ansprüche auf ein Gebiet der Wissensproduktion zu fixieren und zu verteidigen". ${ }^{13}$ Kontrolle, Hierarchie und Autorität stellen somit die Schlüsselwörter für die Charakterisierung eines Typs von Handeln beim Bauen dar, welcher einer Form von Professionalismus entspricht, der durch Erlangung eines privilegierten gesellschaftlichen Status eine spezifische „normative Wissensgrundlage von Experten“ etabliert. Jeremy Till befasst sich eingehender mit dieser Einschätzung und zieht das Folgende in Betracht: Im Entwurfs- und Bauprozess muss die Insistenz auf der von Architektinnen und Architekten ausgeübten Kontrolle und die damit einhergehende Zurückweisung von Zufälligem als Teil der Auferlegung von Ordnung in der Moderne verstanden werden. Dabei basiert diese Auferlegung von Ordnung auf einem Prozess sozialer Kontrolle. Er führt in diesem Zusammenhang die Arbeiten von Zygmunt Bauman an, um zu zeigen, wie sehr sich Ordnung als ein grundlegendes Element für das Verständnis der Entwicklung der modernen Kultur erweist, was dann in diesem Rahmen auch für die Architektur gilt, wie sie heutzutage verstanden wird: ,geordneter Raum stellt Herrschaft dar beherrschten Raum“ und „Herrschaft ist Herrschaft, insofern sie verbietet und ausschließt“. ${ }^{14}$ Entsprechend ,wird deutlich, dass diese Zurückweisung des Zufälligen kein Charakteristikum der Architektur alleine darstellt, sondern der Moderne insgesamt" ${ }^{15}$ Folglich wird Unbestimmtheit im Allgemeinen als ein Risiko oder als eine Schwäche in einem Projekt wahrgenommen, das durch eine Architektin oder einen Architekten kontrolliert werden soll. ${ }^{16}$ Diese Vorstellung von Architektur, oder besser gesagt, dieser Mythos, hat sich ferner über die Jahrzehnte in privilegierter Weise in der Vorstellung des schöpferischen Helden manifestiert, einer Vorstellung, mit der man die Arbeiten von ,Starchitektinnen' und ,Starchitekten' wie Rem Koolhaas, Zaha Hadid oder Norman Foster charakterisiert hat. ${ }^{\mathrm{I}}$

Insofern sie diesen Mythos von Kontrolle, der nicht die wirkliche Architektur, sondern nur ein fiktionales Bild von ihr beschreibt, ${ }^{18}$ ablehnen, möchten Agency-Theorien einerseits eine angemessenere Perspektive auf die Vorgänge anbieten, die sich beim Entwurfs- und Bauprozess tatsächlich abspielen. Darüber hinaus möchten sie auch neue Kanäle der Teilhabe und Zusammenarbeit in diesen Prozessen untersuchen und eröffnen, indem 
einer größeren Anzahl von Akteuren Zugang verschafft wird, und deren Beiträge in einem Kontext, in dem Architektinnen und Architekten lediglich ,ein Antiheld [wären], eine Person, die von Anfang an als Co-Autor fungiert, beziehungsweise eine Person, die aktiv und mit vollem Wissen Autorität aufgibt" ${ }^{19}$ Obwohl nicht alle diese Gesichtspunkte neu sind, ${ }^{20}$ ist jedoch klar, dass sie für viele dieser Autorinnen und Autoren erneut zentrale Bedeutung gewonnen haben im Gefolge der Finanzkrise im Jahr 2008. Die daran anschließende wirtschaftliche Rezession ${ }^{21}$ lieferte erneute Gelegenheit zur Reflexion darüber, wie Bauprozesse ablaufen. Sie untersuchen daher von einer politischen Warte aus Formen der Teilhabe, des Aushandelns und der Transformation der Zusammenarbeit der zahlreichen Akteure, die am Bauprozess teilnehmen. Ferner analysieren sie auch aus dieser Perspektive die Art und Weise, wie unerwartete Wohnformen den ursprünglich intendierten Gebrauch beziehungsweise die Funktionen neu anordnen oder untergraben können.

Agency und Activism Um diesen Formen des Handelns gerecht werden zu können, präsentieren die Forscherinnen und Forscher der Agency-Gruppe an der Universität Sheffield drei unterschiedliche Hauptmodelle für das Verständnis von Agency, in deren Rahmen die Mitwirkenden an ihrem Buch ihre eigenen Vorschläge organisieren:22 Intervenieren, nachhaltiges Agieren und Vermitteln, die alle aus einer aktivistischen Perspektive bestimmt werden. Intervention hat „eine politische und ethische Bedeutung" und geschieht durch Aushandlungs- und Überlegungsprozesse, ,um die Ermächtigung der Betroffenen zu ermöglichen““. ${ }^{23}$ Der Fokus liegt hier jedoch nicht notwendigerweise auf der Intervention aller Nutzerinnen und Nutzer, sondern eher auf den Formen der Agency der Architektinnen und Architekten, die sich die Frage stellen, was sie zur Ermächtigung anderer tun können. Zweitens bezieht sich „nachhaltiges Agieren“ auf einen ethisch verantwortlichen Umgang mit der Umwelt und fokussiert sich wiederum auf die Handlungen und Formen der Agency der Architektinnen und Architekten sowie deren Reaktionen auf aktuelle Herausforderungen der Umwelt, obwohl auch die Vorstellung einer kollektiven Verantwortung für Nachhaltigkeit eröffnet wird. „Vermittlung“ weist schließlich auf eine Art des Handelns hin, das die Entwicklung und Herausbildung unterschiedlicher Formen von Agency ermöglicht. Diese 
Vorstellung von Vermittlung weist eine wichtige Verbindung mit einem der anderen Vorschläge von Jeremy Till auf, wonach die Architektin oder der Architekt als Sinnstifter fungiert, wobei das Ziel von ihrem architektonischem Handeln nicht darin besteht, alle Meinungen zu sammeln und Lösungen für eine Konsensgewinnung anzubieten, sondern eher darin, Design-Prozesse zu eröffnen und auszuhandeln, die durchdrungen sind und transformiert werden von den künftigen Wünschen der Bewohnerinnen und Bewohner: „Wo Problemlösen gemäß einem positivistischem Verständnis dazu tendiert, das Soziale und das Politische entweder auszuschließen oder von ihm abzusehen, befasst sich das Stiften von Sinn ganz unausweichlich damit und stimmt daher mit einem Modell von Agency in der Architektur überein, in dem soziale und politische Fragen ins Blickfeld geraten und dann in räumlichen Diskussionen ausgehandelt werden“" ${ }^{24}$

In diesem Vorschlag von Till liegt der Fokus jedoch hauptsächlich auf dem Entwurfsprozess: ,damit man die volle Kraft der Sinnstiftung erlangen kann, muss man sich der gesamten Vielfalt von Bedingungen widmen, bei denen Entwurf als die Anwendung der architekturalen Intelligenz eine Rolle spielen mag“. ${ }^{25}$ Wie in den Beiträgen des von „The Agency“ herausgegebenen Bandes aber auch in einem bedeutsamen Teil der Agency-Theorien immer wieder an zahlreichen Stellen deutlich wird, wird die Zusammenarbeit und die Teilnahme einer größeren Gemeinschaft hauptsächlich als deren Mitarbeit bei der Planung des Bauprozesses verstanden. In einem beträchtlichen Teil der Projekte, welche als Beispiele für eine Perspektive vorgeschlagen wurden, die neue Formen von Agency eröffnen sollen, wird Intervention und die Teilnahme unterschiedlicher Akteure lediglich im Rahmen der Entwicklung des Bauplans angesprochen: Gelegentlich wird dieser Prozess von den möglichen Nutzern selbst begonnen, und diese suchen nach der Mitarbeit einer Architektin oder eines Architekten, um eine Idee in einen Arbeitsplan umzusetzen, wohingegen es bei anderen Gelegenheiten die Architektinnen und Architekten selber sind, die auf die Teilnahme potentieller Nutzer aus sind. In beiden Fällen findet jedoch der Input durch die neuen Akteure im Rahmen des Planungsprozesses statt und er bleibt ziemlich häufig auf diesen beschränkt, indem der Bauprozess erst im Anschluss auf traditionelle Art und Weise entwickelt wird, die den Instruktionen und Hinweisen der Planung folgt. Ganz in diesem Sinne macht Tomo- 
hiro Sugeta deutlich, dass, ,in den gegenwärtigen Debatten [...] eine Tendenz [besteht], Nutzer lediglich als potentielle Akteure anzusehen, welche die eine oder andere Rolle in Projekten spielen, die von den Architektinnen oder Architekten angeleitet werden“ und ,ihr Nachdruck liegt häufig auf einem partizipatorischen Verständnis der Nutzer beim Design“. ${ }^{26}$ Wo dies der Fall ist, besteht daher das Risiko, einfach eine individuelle Form des Planens (in der nur die Architektin oder der Architekt den gesamten Prozess plant) durch eine weiter gefasste Gruppe und gemeinschaftliches Planen zu ersetzen, bei dem jedoch das Handeln im Bauprozess dieselbe Struktur aufweist und immer noch durch dieselben Vorstellungen von Intention und Kontrolle bestimmt würde, die durch einen Plan festgelegt werden.

Des Weiteren liegt der Nachdruck immer noch ganz klar auf den Handlungsformen des Architekten und der Architektin, wie oben in der Beschreibung der drei Modelle von Agency in Agency. Working with Uncertain Architectures bereits identifiziert wurde, nämlich, wie Architektinnen und Architekten die Partizipation von anderen Formen der Agency begünstigen und möglich machen können, sich die Perspektive dabei aber eindeutig nur auf ihre eigene Rolle konzentriert. Sowohl das Modell der Intervention als auch das der Nachhaltigkeit suchen, wie bereits angedeutet wurde, nach Wegen, wie die Architektin oder der Architekt Autorität abgeben und die Ermächtigung von anderen möglich machen oder wie sie mit der Umwelt besser verantwortungsvollen Umgang pflegen können. Auch im Rahmen des Modells der Vermittlung ist die Perspektive wiederum die der Architektin oder des Architekten, denn diese spielen die Schlüsselrolle als Vermittler zwischen den unterschiedlichen gegenwärtigen Formen der Agency, und sie sind diejenigen, die deren Entwicklung und Konsistenz erleichtern. Dieser Ansatz charakterisiert auch Tills Vorstellung vom Sinnstifter insofern, als Sinnstiftung impliziert, dass dem Handeln anderer Form und Kohärenz verliehen wird, so dass am Ende die Agency von Architektinnen und Architekten durch eine neue privilegierte Position begünstigt wird. In diesem Zusammenhang führt Till den Planungstheoretiker John Forester an: „Sinnstiftung [...] ist eine Angelegenheit der Veränderung, des Respekts, der Anerkennung und des Gestaltens der Lebenswelt der Leute“. ${ }^{27}$ Abschließend lässt sich also festhalten, dass von dieser Warte aus gesehen die Ansichten und Handlungen Anderer respektiert und eingeschlossen 
werden, aber dass dies in den Worten von Schneider und Till großteils eine Frage dessen ist, ,welchen Part die Architektin oder der Architekt spielen könnten oder können“, ${ }^{28}$ um anderen diesen Spielraum zu eröffnen.

Auf der anderen Seite führt Till in seinem Buch Architecture Depends die Vorstellung des ,lockeren Raumes“ (slack space) ein, gerade um Formen der kontrollierenden Rolle von Architektinnen und Architekten zu reduzieren. Dabei handelt es sich um einen Raum, der frei von Überregulierungen und Verplanung ist, und in dem sich Unvorhergesehenes entwickeln kann: Es ist „ein Raum, der , weicher' als derjenige ist, an dessen Stelle er getreten ist, insofern er nicht auf die Prinzipien der Abstraktion, der Normalisierung und der Ordnung gegründet ist, die den harten Raum untermauern. Er beansprucht weder Kontrolle noch Aufteilung in derselben Art und Weise, wie dies beim harten Raum der Fall ist" ". ${ }^{29}$ Er verwendet den Begriff der ,Lockerheit“ (slackness), der von William Connolly skizziert wurde. Dieser hat argumentiert, dass jeglicher gemeinsame Grund nicht durch Ordnung und Regulierungen überbestimmt werden sollte, so dass daher dieser Raum der Unbestimmtheit bei der Aktivität des Bauens sowohl die Ermächtigung unterschiedlicher Akteure als auch die Entwicklung ihres Handelns in ungeplanten Formen begünstigen würde. Wiederum liegt der Fokus dieses Ansatzes jedoch auf Mechanismen, welche von Architektinnen und Architekten zur Entwicklung der Idee des ,lockeren Raumes' im Rahmen dessen eingesetzt werden, was er als ,Lo-Fi Architektur' bezeichnet. In anderen Worten geht es darum, welche Art von Design diese Art von Architektur unterstützt, und weniger um eine Untersuchung der Frage, welche Arten von Handlungen oder Formen der Agency in diesem Kontext entwickelt werden. ${ }^{30}$

Non-human Agency Dies alles macht also deutlich, dass Studien zu Agency in Wahrheit häufig andere Formen von Agency gar nicht ernsthaft in den Blick nehmen, und sich kaum damit beschäftigen, wie diese umgesetzt werden, wie sie sich entwickeln und worin ihr spezifischer Prozess der Zusammenarbeit besteht. Vielmehr konzentrieren sie sich stattdessen auf die Tätigkeiten von Architektinnen und Architekten. Völlig anders sieht jedoch die Perspektive der Agency-Forschung zur Architektur aus, die sich aus der AkteurNetzwerk-Theorie (ANT) entwickelt hat. Ganz im Gegenteil ist Agency in den Arbeiten von Autoren wie Bruno Latour, ${ }^{31}$ Albena 
Yaneva $^{32}$ oder Thomas Gieryn ${ }^{33}$ eine Angelegenheit relationaler Netzwerke von Menschen und Dingen zu, ohne dass dabei einem menschlichen Akteur - gleichviel, ob einem Individuum oder einem Kollektiv - irgendein Primat zugesprochen würde. Latour zieht ganz im Einklang damit in Betracht, dass Intentionalität und Wille keine notwendigen Bedingungen für das Handeln darstellen, und dass diejenigen, die wir als Akteure bezeichnen, seien sie menschlich oder nicht, in Wahrheit Produkte oder Wirkungen von Netzwerken sind und besser als ,actants ' charakterisiert werden sollten, das heißt als etwas, was zum Handeln gemacht wurde. ${ }^{34}$ Damit ist aus dieser Perspektive ,Architektur [...] nicht das Werk von Architektinnen und Architekten“, denn es ist in Wahrheit ,eine Koproduktion des Sozialen und des Formalen, von Menschen und Nicht-Menschlichem und von Bedeutung und Materie“35 und die kreative Tätigkeit entspricht daher einem komplexeren Prozess, zu dem unterschiedliche Assoziationsketten gehören. Yaneva untersucht in diesem Kontext zum Beispiel in The Making of a Building $^{36}$ das Office for Metropolitan Architecture (OMA) von Rem Koolhaas mit einer Methode ähnlich derjenigen, derer sich Latour und Woolgar in Laboratory Life. The Social Construction of Scientific Facts ${ }^{37}$ bedient haben, die Wissenschaft und Technologie betrachten, indem sie Wissenschaftler bei der Arbeit beobachten und analysieren. Sie nimmt das alltägliche Verhalten von Architekten in den Blick, untersucht ihre Beziehungen, Gewohnheiten, Modelle sowie ihre regulierten und allgemein üblichen Praktiken, um „Architektur, während sie geschaffen wird“ statt ,geschaffener Architektur“"38 zu verstehen, und um zu zeigen, dass eine große Anzahl heterogener Akteure in dem Prozess engagiert sind und auf unterschiedliche Weise wieder miteinander verbunden werden: „Gruppen der Gesellschaft, Gravitationsgesetze, historische Gebäude, Architektinnen und Architekten, Erfordernisse der Gebietseinteilung, Straßenbegrenzungen, Museumsphilosophie, Denkmalschützer und Anwohner“.39

Dieser Ansatz führt zu wichtigen Implikationen für die Diskussion über Agency: Eine der wichtigsten Implikationen besteht hier darin, dass er eine repräsentationalistische Konzeption der Architektur vermeidet, indem im Gegensatz dazu die Priorität auf deren performative Aspekte und den Bauprozess selbst gelegt wird, statt auf die Ideen, Absichten oder Pläne. In diesem Sinne gilt, was auch Andrew Pickering deutlich macht: ,,[D]er attraktivste Charak- 
terzug des Akteur-Netzwerk-Ansatzes besteht genau darin, dass seine Anerkennung materieller ,Agency“ uns helfen kann, sich dem Bann der Repräsentation zu entziehen. Er weist einen Weg $\mathrm{zu}$ einem durchgreifenden Wandel in das performative Idiom“.40 Auf der anderen Seite besteht eine weitere Hauptkonsequenz dieses Ansatzes in seinem Gegensatz zu den oben angesprochenen Arbeiten von ,The Agency“ oder von Till und Schneider: Anstatt sich auf die Position der Architektin oder des Architekten zu fokussieren, lässt hier die symmetrisch verstandene Agency zwischen Menschen und Nicht-Menschlichem, und der Umstand, dass beide als ,actants ' in einem Netzwerk verstanden werden, keinen echten Raum für die Intentionen der beteiligten Menschen, so dass kritische menschliche Intentionalität und politisierte Praxis weitestgehend eliminiert werden. ${ }^{4 \mathrm{I}}$ Wie Lambros Malafouris deutlich gemacht hat, sind in ANT „Macht, Intentionalität und ,Agency“ [...] keine Eigenschaften der isolierten Person oder der isolierten Sache, sie sind Eigenschaften einer Assoziationskette" ${ }^{\text {"42 }}$ und so werden die einzelnen und individuellen Formen des Handelns unter die Perspektive des Netzwerks subsumiert. Einerseits bedeutet dies nicht nur, dass die Vorstellung der Autorschaft nicht mehr als das Zentrum des architektonischen Handelns fungieren kann, sondern vielmehr, dass sie in der Praxis ihre gesamte Sichtbarkeit verliert, was wiederum eine genauere Untersuchung und Analyse der anderen Formen von Agency erfordert. Andererseits werden aber die Formen der individuellen Intention oder Initiative einer ziemlich schwer zu erklärenden Position überlassen, was dann auch deren Möglichkeiten betrifft, Veränderungen oder politische Transformationen herbeizuführen.

Daher haben wir es auf der einen Seite mit Untersuchungen zur Agency zu tun, die einem ähnlichen Pfad folgen, wie dem, der von der Gruppe „The Agency“ oder von Forschenden wie Schneider oder Till eingeschlagen wurde, und denen es primär darum geht, die Intentionen und Formen der Kontrolle durch Architektinnen und Architekten in unserer Konzeption der Bautätigkeit drastisch herunterzuspielen, und so neue Wege der Ermächtigung für alle Akteure zu eröffnen, die im Bauprozess involviert sind. Diese Perspektive hat dann ganz eindeutig ihren Fokus auf der Profilierung neuer Rollen für Architektinnen und Architekten, welche diejenigen sind, die die Entwicklung solcher Formen von Ermächtigung erlauben würden. Dies hatte zur Folge, dass dieser Ansatz 
sich bislang nicht auf eine ausgearbeitete Analyse der spezifischen Performanz jener anderen neuen Formen von Agency konzentrieren konnte, nämlich eine Analyse, die deren konkrete eigentümliche Formen des Handelns und der wechselseitigen Beziehungen erhellen könnte. Andererseits wiederum bietet die Analyse von Formen der Agency aus der ANT-Perspektive ziemlich spezifische Untersuchungen von allem, ob menschlich oder nicht, was in unterschiedlichen Phasen oder Abschnitten des Entwurfs- und Bauprozesses interveniert. Indem jedoch die Perspektive des Netzwerks und der Assoziationen betont wird, wird jegliche Form individueller Intention tatsächlich verwischt, und damit verlieren die Vorstellungen von politischer Intervention und jeglicher Form von Autorschaft ihren Belang und spielen damit im Entwurfs- und Bauprozess keine wirklich relevante Rolle mehr.

Aus all diesen Gründen besteht eine der hauptsächlichen Herausforderungen in der gegenwärtigen Forschung zu Agency in der Architektur darin, die Leistungen beider dieser Ansätze miteinander zu verbinden, dabei aber im Einzelnen deren jeweils problematische Aspekte zu vermeiden. Die Aufgabe besteht hier dann darin, eine Konzeption von Agency zu entwickeln, die I) fähig ist, Handeln und seine Kontrolle und Verantwortlichkeit auf all jene zu verteilen, die am Bauprozess teilnehmen, die 2) auch die Verteilung von Kreativität auf den gesamten Prozess erlaubt, und sie nicht nur auf die ursprüngliche Inspiration oder einen exklusiven Plan beschränkt, d.h. ein Konzept von Agency, welches den gesamten Handlungsverlauf ernst nimmt und entsprechend auf spezifische Formen der Intervention aller involvierten Parteien fokussiert, und das 3) sowohl materielle nicht-menschliche Agency berücksichtigt, jedoch individuelle Intentionen, Initiativen und Erwartungen nicht vollständig eliminiert. Mit diesem Ziel vor Augen könnten weitere Untersuchungen innerhalb der Handlungstheorie über ungeplante und nicht vollständig kontrollierte oder hierarchisch definierte Aktivitäten innerhalb der Architekturtheorie einen signifikanten Beitrag leisten zu einem genaueren und weiterentwickelten Verständnis vieler unterschiedlicher Formen von Agency (und ihrer Strategien, Formen der Zusammenarbeit, Begrenzungen und so weiter), die in Bauprozessen eine Rolle spielen. ${ }^{43}$ 
Anmerkungen

1 Vgl. Agency in Architecture. Reframing Criticality in Theory and Practice, Footprint 4, 2009; Agency and the Praxis of Activism, Field Journal 3 (1), 2009, www.field-journal.org [14.06.2019]; Architectural Research Quarterly 13 (2), 2009 und Perspecta: Agency 45, 2009.

2 Florian Kossak, Doina Petrescu, Tatjana Schneider u.a. (Hg.), Agency. Working with Uncertain Architectures, London 2009.

3 Nishat Awan, Tatjana Schneider und Jeremy Till, Spatial Agency. Other Ways of Doing Architecture, Oxon/UK 2011.

4 Jeremy Till und Tatjana Schneider, „Beyond Discourse. Notes on Spatial Agency", in: Agency in Architecture, Footprint 4, 2009, S. 97-111.

5 Ana Paula Baltazar und Silke Kapp, „Against Determination, Beyond Mediation", in: Kossak, Petrescu, Schneider u.a. (Hg.), Agency, (Anm. 2), S. 131.

6 Till und Schneider, „Beyond Discourse. Notes on Spatial Agency” (Anm. 4), S. 97.

7 Vgl. Bruno Latour und Albena Yaneva, „Give me a Gun and I Will Make All Buildings Move: An ANT's View of Architecture", in: Reto Geiser (Hg.), Explorations in Architecture. Teaching, Design, Research, Basel 2008, S. 81.

8 James Faulconbridge, "The Regulation of Design in Global Architecture Firms. Embedding and Emplacing Buildings", Urban Studies 46, 2009, S. 2537-2554.

9 Rob Imrie und Emma Street, „Regulating Design. The Practices of Architecture, Governance and Control", Urban Studies 46, 2009, S. 2507-2518.

10 Peter Kraftl, „Utopian Promise or Burdensome Responsibility? A Critical Analysis of the UK Government's Building Schools for the Future Policy" , Antipode 44, 2012, S. 847-870.

11 Vgl. Beth Preston, A Philosophy of Material Culture, New York 2013, S. 15-43.

12 Genau aus diesem Grund haben Tim Ingold und Elisabeth Hallam die Charakterisierung von Kreativität als Innovation abgelehnt, weil diese nur auf die Ergebnisse oder die Produkte der Kreativität fokussiert und nicht auf die Kreativität selbst, die sie als Improvisation beschreiben. Vgl. Elisabeth Hallam und Tim Ingold (Hg.), Creativity and Cultural Improvisation, Oxford, New York, 2007. 
13 Vgl. Colin Lorne, "Spatial Agency and Practising Architecture Beyond Buildings", Social and Cultural Geography 18, Nr. 2, 2017, S. 274.

14 Zygmunt Bauman, Wasted Lives. Cambridge 2004, S. 31 (zitiert nach Jeremy Till, "Architecture and Contingency", Architecture \& Indeterminacy, Field Journal 1, 2008, S.120-135).

15 Till, "Architecture and Contingency" (Anm. 14), S. 120.

16 Vgl. Kim Trogal und Leo Care, „A Quick Conversation About the Theory and Practice of Control, Authorship and Creativity in Architecture", Architecture \& Indeterminacy, Field Journal 1 (1), 2008, S. 136-145.

17 Vgl. Till und Schneider, „Beyond Discourse. Notes on Spatial Agency" (Anm. 4), S. 97.

18 Vgl. Till: „Architecture and Contingency” (Anm. 14), S. 135.

19 Till und Schneider, "Beyond Discourse. Notes on Spatial Agency" (Anm. 4), S. 97.

20 Natürlich liefen Projekte dieser Art im Verlauf der Geschichte auch parallel zur kanonischen Geschichte der Architektur ab. Eine beachtliche Reihe von Beispielen dafür stellen die Projekte und Gruppen aus unterschiedlichen Zeiten und Kontexten dar, die als Fälle von räumlicher ,Agency' in dem Buch Spatial Agency (Anm. 3) aufgeführt werden.

21 Vgl. z. B. Kurt Evans, Iben Falconer und lan Mills, "Letter from the Editors", Perspecta: Agency, 45, 2009, S. 3, und Lorne, "Spatial Agency and Practising Architecture Beyond Buildings" (Anm. 13), S. 276.

22 Vgl. Kossak, Petrescu, Schneider u.a. (Hg.), Agency. Working with Uncertain Architectures (Anm. 2), S. 1-18.

23 Ebd., S. 6.

24 Jeremy Till, Architecture Depends. Cambridge, Mass. 2009, S. 168.

25 Ebd.

26 Tomohiro Sugeta, Agency. Enabling or Constrained? Thinking Agency in Architecture Through the Built Works of Rem Koolhaas, MA Thesis, University of Westminster, London 2014, S. 17.

27 Till, Architecture Depends (Anm. 24), S. 168.

28 Schneider und Till, "Beyond Discourse. Notes on Spatial Agency" (Anm. 4), S. 100.

29 Till, Architecture Depends (Anm. 24), S.133.

30 Trotz alledem eröffnet das Konzept des lockeren Raums ein neues Feld für unvorhergesehene Aktivitäten, und viele der Projekte, die 
von der Warte dieser Idee oder von der Vorstellung von Unbestimmtheit aus Spatial Agency von Awan, Schneider und Till (sowohl in ihrem Buch als auch auf der Webseite) aus betrachtet werden, bieten eine gute Gelegenheit diese anderen Arten von ,Agency' und deren Strategien weiter zu untersuchen.

31 Vgl. z.B. Latour und Yaneva, „Give me a Gun and I Will Make All Buildings Move. An ANT's View of Architecture" (Anm. 7), S. 80-89.

32 Vgl. Albena Yaneva, The Making of a Building. A Pragmatist Approach to Architecture, Oxford u.a. 2009.

33 Thomas Gieryn, "What Buildings Do", Theory and Society, 31, (1), 2002, S. 35-74.

34 Bruno Latour, Reassembling the Social. An Introduction to ActorNetwork Theory, Oxford 2005, S. 46 [Deutsch: Eine neue Soziologie für eine neue Gesellschaft: Einführung in die Akteur-NetzwerkTheorie. Aus dem Engl. von Gustav Roßler, Frankfurt a. M. 2010].

35 Kjetil Fallan, "Architecture in Action. Traveling with Actor-Network Theory in the Land of Architectural Research", Architectural Theory Review, 13 (1), 2008, S. 80-96, (S. 90).

36 Vgl. Yaneva, The Making of a Building. A Pragmatist Approach to Architecture (Anm. 32).

37 Bruno Latour, und Steve Woolgar, Laboratory Life. The Social Construction of Scientific Facts, London 1979.

38 Yaneva, The Making of a Building. A Pragmatist Approach to Architecture (Anm. 32), S. 197.

39 Ebd., S.198.

40 Andrew Pickering, The Mangle of Practice, Chicago 1995, S. 13.

41 Vgl. Lorne, "Spatial Agency and Practising Architecture Beyond Buildings", (Anm. 13), S. 271. Vgl. auch Noel Castree, "False Antitheses? Marxism, Nature and Actor-Networks", Antipode, 34, S. 111-146.

42 Lambros Malafouris, How Things Shape the Mind. A Theory of Material Engagement, Cambridge, MA 2014, S. 129.

43 Eine erste Fassung dieses Textes wurde als Abschnitt veröffentlicht in Ana García Varas, "Agencies in Architecture. Intention and Improvised Action in the Building Process" in: A. Arteaga ( $\mathrm{Hg}$.), Architectures of Embodiment: Unfolding Fields of Intelligibility, Zürich, Berlin, 2020. Dieser Text untersucht, wie ein alternatives Modell des Handelns wie die Improvisation uns dabei helfen kann, die unterschiedlichen Formen von ,Agency' sowie Arten ungeplanter kollektiver Aktivität, die im Bauprozess eine Rolle spielen, 
präziser zu definieren. Der vorliegende Text ist von Alexander Staudacher aus dem Englischen übersetzt worden. 


\section{Manifeste, Diskurse, Chatter Sprache als Medium der Architektur}

Zunächst: Eine ,sprechende' Fotografie Die Fotografie, die anlässlich der Vergabe des Pritzker-Preises 2016 vom Geehrten, dem chilenischen Architekten Alejandro Aravena, in vielen Medien und auf der Website des Auslobers veröffentlicht wurde, ist mehr als nur eine fotogene Aufnahme des Preisträgers. ${ }^{I}$ Sie zeigt Aravena vor einer mit Skizzen und Notizen bedeckten Wand stehend, den Betrachter direkt anblickend. Die im Bild ausgedrückte kreative Programmatik ist klar: Wo sich frühere Architekten gedankenversunken am Reißbrett (Hannes Meyer), repräsentativ mit imposantem Modell (Ludwig Mies van der Rohe) oder als professionelle Clique im informellen Gespräch (Frank O. Gehry, Jean Nouvel und, mit den Beinen auf dem Tisch, Rem Koolhaas) fotografieren ließen, da präsentiert sich Aravena als Teil eines gestalterischen Kollektivs - des Büros Elemental, wie der Schriftzug auf der Wand verrät. Dessen Arbeit wird in den skizzenhaften Grundrissen und Ansichten von Bauprojekten als vielgestaltiger, kleinteiliger und offener Prozess charakterisiert. Die Größe der Darstellung an der Wand und ihr Entwurfscharakter suggerieren eine halböffentliche Vorstellung und Diskussion, vermutlich mit den künftigen Bewohnern der in Planung befindlichen Einfamilienhäuser, die allesamt unterschiedlich gestaltet sind. Sprache ist auf dem Papierbogen vor allem in kleinen Notaten enthalten. Die Vorstellung gesprochener Sprache drängt sich dennoch beim Betrachten des Fotos unmittelbar auf, da sich die flüchtig gezeichneten Entwürfe in eine imaginierte Situation der kommunikativen Vermittlung einfügen, in welcher der planende Architekt nur einer unter vielen Akteuren und Akteurinnen ist. Die Fotografie kann somit als Ausgangspunkt der folgenden Überlegungen zur Medialität der Sprache in archi- 
tektonischen (Entwurfs-)Zusammenhängen dienen. Die Rolle und Funktion von Sprache als ein Medium im kreativen Prozess und in dessen Vermittlung haben sich dabei historisch grundlegend gewandelt: Medial von der geschriebenen zur zeichenhaft verbildlichten Sprache, zum gesprochenen Wort und zur digital vermittelten Produktion von Texten aller Art. Inhaltlich vollzog sich eine Wandlung vom normativen Sprachgebrauch in Traktaten und Manifesten über die Verschwisterung mit der Literatur seit dem I9. Jahrhundert; über informationstheoretische und linguistische Impulse seit den I96oer Jahren bis hin zum Paradigma der Diskursivität in der Gegenwart. Der Beitrag möchte diese Entwicklungen schlaglichtartig nachzeichnen und fragt nach der spezifischen, performativen Leistung geschriebener und gesprochener, theoretischer und fiktionalisierender Sprache in gegenwärtigen architektonischen Entwurfs- und Arbeitsprozessen.

Sprache als Medium der Architektur Auch wenn die „Baukunst [...] nicht in Worten, sondern in einer sichtbaren und handgreiflichen Demonstration“ besteht, wie Elisabeth Kieven ihren Beitrag zu einem Sammelband über die „Medien der Architektur“ einleitet und damit den barocken Architekten Georg Andreas Böckler zitiert, so ist die Sprache doch ein ganz grundlegendes Medium der Architektur - auch Böckler machte sich letztlich weniger als Praktiker denn als Übersetzer Palladios einen bleibenden Namen. ${ }^{2}$ Im erwähnten Sammelband werden demnach auch sprachliche Äußerungsformen als mediale, also vermittelnde Instrumente der Baukunst gleichberechtigt neben bildlichen Formen - Zeichnung, Fotografie, Modell, Diagramm etc. - thematisiert, ebenso die Architekturtheorie, -beschreibung, -kritik und das hybride Format der Ausstellung. Wie und was gebaut wird, und wie das Gebaute gesellschaftlich wahrgenommen, kulturell verhandelt und für die Gegenwart und Zukunft interpretiert wird, dafür spielen sprachliche Verständigungsprozesse, seien sie mündlicher oder schriftlicher Natur, eine wesentliche Rolle.

Im Folgenden soll der Blick vor allem auf jene im weiteren Sinn sprachlichen Äußerungsformen gerichtet werden, die die Entwurfsprozesse von Architektinnen und Architekten begleiten sowie ihre Selbstdarstellung und gesellschaftliche Wahrnehmung prägen. Bis zum Aufkommen des Internets war die geschriebene Sprache das wichtigste Medium, um ästhetische und technische, 
künstlerische und handwerkliche Aspekte des Bauens zu systematisieren und zu vermitteln, die eigene Praxis öffentlichkeitswirksam zu profilieren und zuallererst den Entwurfsprozess zu konzeptualisieren. Das allen sprachlichen Äußerungsformen innewohnende Moment der Performativität erhält im Zeitalter des Internets durch die audiovisuelle Komponente einerseits, die Instantaneität der Vermittlung andererseits, neue Konturen und Potenziale. Historisch ist das Verhältnis von Architektur und Sprache in eine lange Tradition wechselseitiger Bezugnahmen und Aneignungen eingebettet. Nicht erst, aber auf besonders fruchtbare Weise sind seit der Moderne bestehende oder erfundene Bauten wichtige Bezugspunkte der literarischen Imagination, während sich im 20. Jahrhundert bedeutende Architekten und Architektinnen immer wieder von Texten und Fiktionen inspirieren ließen.

Architektur in der Literatur, Literatur in der Architektur Die sprachliche Darstellung architektonischer und städtebaulicher Orte in literarischen Texten geht seit den antiken Ekphrasen oft weit über bloße Beschreibungen hinaus. ${ }^{3}$ In der kontrastierenden Gegenüberstellung mit der ausführlich geschilderten Pracht des Herrscherpalasts Alkinoos' etwa stellt Homer die existenziellen Entbehrungen des irrfahrenden Odysseus umso deutlicher heraus: „Gleich dem Strahle der Sonn' und [...] dem Schimmer des Mondes [...] so reichlich schmückten Alkinoos' Wohnung die Götter. Lange stand bewundernd [...] Odysseus. Und nachdem er alles [...] bewundert, eilet' er über die Schwell' und ging in die strahlende Wohnung. "4 So wie hier der überirdische Prunk des Herrscherbaus für eine ideale, harmonische Menschengemeinschaft steht, so kommen auch in den biblischen Architekturbeschreibungen des Turms zu Babel oder des Tempels Salomos jene gleichnishaften gesellschaftlichen Analogiebildungen zum Tragen, die auf der übergeordneten kulturellen Bedeutung der Baukunst als ,Mutter aller Künste“ gründen. Literarische Beschreibungen geben somit Auskunft über die gesellschaftliche Funktion und symbolische Ausstrahlung der Architektur. Wo diese einen Verweis auf ein erlösendes Reich des Überirdischen und Jenseitigen enthielt, wie im mittelalterlichen Kirchenbau, da priesen zeitgenössische Autoren die Bauten als Räume des Lichts. Und wo solche Glaubenssicherheiten verloren schienen, da verkehrten sich die Kategorien, wie in der von François Rabelais ersonnenen und detailreich beschriebenen Abtei 
Thélème, die als Bau einer weltlichen Glaubensgemeinschaft des freien Willens der prachtvollen Typologie der französischen Renaissance-Schlösser folgt. 5

Rabelais' Romanzyklus aus dem I6. Jahrhundert imaginiert in der maßgenauen Schilderung der fiktiven Abtei ein Reich der Schönheit, Bildung und Freiheit. In der Literatur der Moderne lässt sich dann eine solche konstruktiv semantisierende Schilderung architektonischer Formen und Prinzipien - jenseits ihrer bloßen Beschreibung als Schauplatz oder Motiv - vermehrt aufspüren, worauf die jüngere literaturwissenschaftliche Forschung reagiert hat. ${ }^{6}$ Die psychische Dimension des gebauten Raums rückte zusehends ins literarische Bewusstsein. Die Entdeckung der seelischen Innerlichkeit durch die beginnende Psychologie verlief parallel zur fortschreitenden Industrialisierung und zur städtischen Verdichtung. Wenn am Ende des I9. Jahrhunderts der deutsche Autor Hermann Conradi seinen neurasthenischen Roman-Protagonisten eine „fürchterliche Neugier auf die Farbe seiner Tapete“ in dessen Arbeitszimmer verspüren lässt (,nervöses Prickeln in den Fingern, es trieb und zwang ihn unwiderstehlich, die Tapete zu betasten [...] er krallte die Fingernägel ein und kratzte gerippte Fetzen herunter $[\ldots]^{“ 7}$ ), dann verknüpft er nicht nur das gerade erst entstehende narrative Konzept des stream of consciousness mit der Topik des Interieurs als un-heimlicher Ort der Innerlichkeit. Die kleine Welt des mit den Händen erforschten Arbeitszimmers steht in ihrer partikularen Perspektivik vielmehr auch für den ,,Verlust eines einheitlichen Weltverhältnisses“. ${ }^{8}$ Den großen naturwissenschaftlichen Gedankengebäuden und ästhetischen Systematiken des I8. und I9. Jahrhunderts wird nunmehr mit Skepsis und Ironie begegnet. Der Philosoph Friedrich Theodor Vischer spricht denn auch von dem ,oberen und unteren Stockwerk“, um das Verhältnis von sittlicher Weltordnung und profaner, entfremdender Alltagserfahrung zu reflektieren. Zu Vischers Etagenmodell ließen sich zahlreiche weitere Beispiele hinzufügen, wie seit dem vorletzten Jahrhundert gebaute Räume als ästhetische Projektions- und Denkräume (im Fall Vischers auch als epistemologische Metaphern) angeeignet und interpretiert wurden. Ein bekanntes Exempel der jüngeren Zeit ist das imposante mittelalterliche ,edificio" in Umberto Ecos Roman Der Name der Rose (I980) - auch hier als epistemologische Metapher. 9 
Umgekehrt haben sich vor allem seit den I960er Jahren zahlreiche Architekten und Architektinnen nicht nur von literarischen und philosophischen Texten inspirieren lassen, wie etwa Peter Zumthor im Projekt der Poetischen Landschaft oder - unter ganz anderen Vorzeichen - Peter Eisenman in der Zusammenarbeit mit Jacques Derrida. Vielmehr hat der ,Linguistic Turn“ in den Geistesund Sozialwissenschaften eine grundlegende Hinwendung zu den gesellschafts- und kulturprägenden Facetten von Sprache und Schrift gezeitigt, im Zuge derer auch die Architektur zunehmend als zeichnerische und gebaute Artikulation verstanden wurde. Sprechen im Verhältnis zum Handeln, aber auch die Materialität von Sprache und Schrift rückten dabei in den Fokus. ${ }^{10}$ Wenn zudem, wie Hayden White argumentiert, ${ }^{11}$ die Darstellung oder Erzählung von Geschichte stets als Interpretation derselben zu verstehen ist, dann rückt auch die Idee von Architektur als historisch bedingte und medial verfasste Form in den Fokus: Diese folgt nicht mehr primär einer konkreten Funktion, sondern ,spricht' für sich, erscheint ästhetisiert, als narrativierender oder fiktionalisierender Geschichts- und Ortsbezug, oder ,kommuniziert' mit Umgebung und Betrachtern. In Ableitung von der linguistischen Analyse der doppelten Natur des sprachlichen Zeichens - als Verbindung von Form und Inhalt oder Bedeutung - entfaltet die Semiotik in der architektonischen Praxis und Theorie der I96oer Jahre eine bemerkenswerte Wirkung, in der die Architektur zur ,Sprache " wird. ${ }^{12}$

"From manifesto to discourse"? Die erwähnten Projekte von Zumthor und Eisenman lassen sich als Beispiele dafür anführen, wie sprachliche und gestalterische Artikulation in ein kommunikatives Verhältnis gesetzt werden. Das Medium der Schrift im Literarischen findet in Zumthors Architektur als physisches Raumgefüge eine Entsprechung. Die poetische Beschreibung der umgebenden Landschaft durch Schriftsteller und Schriftstellerinnen wird gerahmt von der architektonischen Reflexion auf sowohl jene Gedichte wie auf die Natur selbst - so die Idee von Zumthors Projekt der Poetischen Landschaft. ${ }^{13}$ Peter Eisenman wollte demgegenüber für Jacques Derridas auf Platon referierendes Konzept der ,Chora' eine architektonische Analogie finden. ${ }^{14}$ Im Nebeneinanderstellen der je spezifischen Eigenschaften sprachlicher und baulicher Ausdrucksmöglichkeiten tritt der Aspekt ihrer Medialität deutlich hervor - ein Begriff, für den Medientheorie und -philo- 
sophie im Übrigen noch keine verbindliche Definition gefunden haben. Vereinfachend lässt sich die neuere Literatur zum Begriff der Medialität dahingehend zusammenfassen, dass mit ihm mehr als die bloßen Eigenschaften eines Mediums bezeichnet werden. Medialität kann vielmehr verstanden werden als jene Dimension im menschlichen Umgang mit vermittelten Zeichen, in der diese nicht nur übertragen, sondern unter jeweils anderen Bedingungen stets aufs Neue als Sinninhalte generiert werden. Die kommunikative Funktion der Medien wird somit um eine epistemische Dimension erweitert: wie wir Dinge wahrnehmen und begreifen, hängt wesentlich von den medialen Bedingungen unserer Begegnung mit ihnen ab. ${ }^{15}$

Entsprechend stehen auch die gesellschaftliche Wahrnehmung und die kulturelle Bedeutung von Architektur in Korrelation zu den Bedingungen ihrer medialen Vermittlung. Die geschriebene Sprache ist zumeist eine Sprache der beschreibenden, behauptenden, im Manifest auch der erfindenden Worte. Im Architekturtraktat, das mit der Einführung des Buchdrucks in der Renaissance zum wichtigsten architekturpraktischen wie -theoretischen Medium avanciert, werden nicht nur historisches Wissen und ästhetische Wertmaßstäbe verbreitet, sondern auch praktische Bauanleitungen gegeben, wobei es zu einer Durchdringung von sprachlichen und bildlichen Elementen in Gestalt von Plänen und Schemata kommt. ${ }^{16}$ Der Textsorte eignet insofern performativer Charakter, sie leitet die Baumeister zum konkreten Handeln an. Performativ ist auch der appellative Gestus des architektonischen Manifests, das als öffentliche Absichtserklärung ästhetische Ziele durchsetzen will. Das Manifest, das im 20. Jahrhundert das Traktat als wichtigste architekturbezogene Textform verdrängt, ist ein Medium des praktisch handelnden Architekten, aus dem Anspruch auf praktisches Bauen heraus formuliert - Bruno Taut, Le Corbusier, Ludwig Mies van der Rohe, Rem Koolhaas sind nur einige berühmte Beispiele dafür. Das Manifest reflektiert politisch-gesellschaftliche Umbruchsmomente innerhalb einer ansonsten (mehr oder weniger) kohärenten Geschichtswahrnehmung, auf die gestalterisch und auf gesellschaftlich relevante Weise reagiert werden soll. Aus tiefer Überzeugung in apodiktischem, manchmal aggressivem Ton verfasst, ist das Manifest nicht auf Dialog ausgerichtet. Seine ästhetische Programmatik erscheint alternativlos: das Wort ist eine ästhetische Setzung wie eine Zeichnung auf Papier. Ange- 
sichts einer solchen Kompromisslosigkeit schien mit der poststrukturalistischen Dekonstruktion der Zeichen und der Analyse der Diskurse das Ende des Manifests als dominante Textform in der Architektur eingeläutet zu sein. Entsprechend konstatiert Anthony Vidler einen kulturellen Übergang „from manifesto to discourse“ seit den I960er Jahren. ${ }^{17}$ In Anbetracht rasanter technologischer Entwicklungen, ökonomischen Wachstums und wissenschaftlichen Fortschritts gerät die Architektur, so Vidler, unter Legitimationsdruck. Ihr Anspruch als relevante kulturelle Ausdrucksform sei kaum unabhängig von den gesellschaftlichen Gegebenheiten realisierbar. Objekthaften Ausdruck für die „Suche nach (Quasi-)Autonomie und [legitimierenden] neuen Leitideen [...] in einer völlig neuen heterogenen Welt" ${ }^{\text {"18 }}$ lieferte der ,Miniature Boom“ ab den I950er Jahren, als kleinformatige Präsentationsmodelle zur unverzichtbaren Voraussetzung architektonischer Großprojekte avancierten. ${ }^{19}$ Die bildhaften Modelle wurden zum wichtigen Medium auch dort, wo eine starke eigene Textproduktion weiter anhielt, wie bei Peter Eisenman, der I976 in New York die Ausstellung Idea as Model organisierte, die den konzeptuellen Eigenwert des Architekturmodells gegenüber der Zeichnung untermauerte. Die Textproduktion geriet zu dieser Zeit, so Vidler, in ein Geflecht von Ansprüchen und Erwartungshaltungen: „Interpretation, historical examination, analysis, and quasi-philosophical exploration replace the short and sharp manifesto. Revolutionary stridency has given place to a worry about the right way to do architecture not seen since the late nineteenth century. “20

Manifeste und Traktate beanspruchen eine Autorität, die freilich letztlich doch eher selten in tatsächlich Gebautem eingelöst wird. Gleichwohl macht Beatriz Colomina eine grundlegende Abhängigkeit der gebauten Architektur von der in den Manifesten ihrer Erbauer eingeforderten Ästhetik geltend: Architekten wie Le Corbusier oder Mies hätten vor der Publikation ihrer Programmschriften ,nicht existiert“. ${ }^{21}$ Damit bezieht sich Colomina einerseits auf die Macht des gedruckten Wortes (und Bildes) in Zeiten geringer Bautätigkeit und prosperierender Printmedien. Das Potenzial des geschriebenen Wortes reicht aber weit über das Gedruckte hinaus: ,[Le Corbusier] became known as an architect and created a clientele for his practice through [L'Esprit nouveau]. In that sense it can be argued that Le Corbusier was an effect of a set of manifestos." ${ }^{22}$ Dabei lassen sich allerdings in der Regel eher kleinere 
Bauaufgaben wie Pavillons als ,gebaute Manifeste' beschreiben, etwa Mies' Barcelona Pavillon - sowie die darauf Bezug nehmende Casa Palestra von OMA auf der Mailänder Triennale von I986. Während mit den großen, wirkmächtigen Manifesten der klassischen Avantgarden der publizierende Architekt als mehr oder weniger solitäre Figur auftritt, entfaltet sich in den I96oer Jahren - von Guy Debord über Buckminster Fuller zu Archizoom und Superstudio - ein reger publizistischer Diskurs mittels ,manifestolike statements“, wie es Vidler formuliert, wohingegen Colomina von „many manifestos“ spricht. ${ }^{23}$ Sie sieht insofern auch in dem nicht mehr apodiktischen, sondern eher diskursiven (, soften") Duktus geschriebener (und in Gestalt von Pavillons gebauter) Statements sowie im medialen Umbruch durch das Internet nicht das Ende, sondern eine Transformation des architektonischen Manifests gegeben: ,The arrival of this new kind of soft manifesto might be the endgame of the twentieth-century manifesto the endgame of print and pavilions as the vehicles for the architectural manifesto. In an age in which electronic media is a primary site of debate and exhibition, new forms of manifesto are surely emerging."24 Irgendwo zwischen Manifest und diskursiver Plattform changiert in dieser frühen Umbruchszeit - I995 - das von Rem Koolhaas zusammen mit Bruce Mau publizierte Schwergewicht $S, M, L, X L$. Es war eine Standortbestimmung des ,chaotischen Abenteuers“"25 Architektur in einer immer stärker vernetzten, immer globaler organisierten Öffentlichkeit, eine überbordende Text- und Bilderfülle, , an amalgam that makes disclosures about the conditions under which architecture is now produced. " ${ }^{26}$ Koolhaas' ehemaliger Mitarbeiter Bjarke Ingels ging pragmatischer vor, als er in seinem „Archicomic“ Yes is More (2009) kreative Entscheidungen und ökonomische Zwänge seines Büros in Comicform darstellte - was viele Rezensenten nicht davon abhielt, von einem (wenn auch spielerischen) ,Manifest' zu sprechen. ${ }^{27}$

Offene Kanäle und der ,Choral Architect' Ausdrücklich manifestartig angelegt war demgegenüber das publizistische Projekt Open Source Architecture, das seit 20I I als Wikipedia-Eintrag, Domus-Artikel, Ausstellungsbeitrag und schließlich in Buchform erschien. ${ }^{28}$ Bemerkenswert ist hierbei weniger die Tatsache, dass über diese verschiedenen Kanäle unterschiedliche Rezipientengruppen angesprochen wurden (die Leser des Wikipedia-Eintrags 
mögen trotz größerer Reichweite im Internet einer relativ kleinen Community entstammen, während mittels der Buchankündigung des Verlags eine potenziell breitere Leserschaft von der Existenz des Projekts erfährt). Wesentlich erscheint vielmehr, dass mit den verschiedenen medialen Realisierungen des Textes sich dieser veränderte, neue inhaltliche Aspekte hinzukommen und andere wegfallen konnten, der Text selbst somit performativ wurde. Von Carlo Ratti als Open Source Projekt auf Wikipedia initiiert und auch in der Buchform als kollaborative Unternehmung deklariert, zeigte sich der performative Charakter am deutlichsten in der Realisierung als Beitrag zur Ausstellung Adhocracy auf der Design Biennale Istanbul 20I2. ${ }^{29}$ Ein hängender Plotter übertrug hier die digitalen Daten des Wikipedia-Eintrags auf eine große Wand im Treppenhaus des Ausstellungsgebäudes, überschrieb online vorgenommene Veränderungen und begann von neuem, sobald der Text vollendet war (Abb. I). In Echtzeit wurde damit der Wikipedia-Text in einen Schreibakt übersetzt, bildete sich die Sprache der OnlineKommunikation im (halb-)öffentlichen Raum der Ausstellung ab.

Das Open Source Konzept war einerseits für die Genese dieses Textes grundlegend (auch wenn die Beitragenden auf die Einladung Rattis reagierten und somit nur ein relatives Maße an Offenheit gegeben $\operatorname{war}^{30}$ ), andererseits bezog es sich auch und vor allem auf eine neue Vorstellung von zeitgemäßer, gesellschaftlich, ökonomisch und ökologisch bewusster Baukultur. So plädierten die Autoren des Buches für eine Konzeption von Architektur, die den modernistischen solitären Baumeister verabschiedet und eine kollaborative, vernetzte, inklusive Methodologie, Open Access-Wissenskultur und Crowdsourcing propagiert. Der autoritären Figur Le Corbusier stellte das Buch den ,Choral Architect entgegen und bezog sich dabei auf N. John Habrakens partizipative Wohnbauprojekte, die seit den I96oer Jahren in den Niederlanden entstanden. Die aus Strukturalismus und Diskursanalyse abgeleitete Idee der Mitbestimmung als Fundament der Open Source Architektur wurde somit unter heutigen medialen Bedingungen aktualisiert. Neu war den Autoren zufolge ein (abermals) verändertes Verhältnis zwischen den Akteurinnen und Akteuren in vernetzten Entwurfs- und Planungsprozessen. Durch die stärkere Instantaneität und größere Vielstimmigkeit der Kommunikation und nicht zuletzt durch das dem Internet zugesprochene (wenn auch inzwischen fragwürdig gewordene) demokratische Poten- 


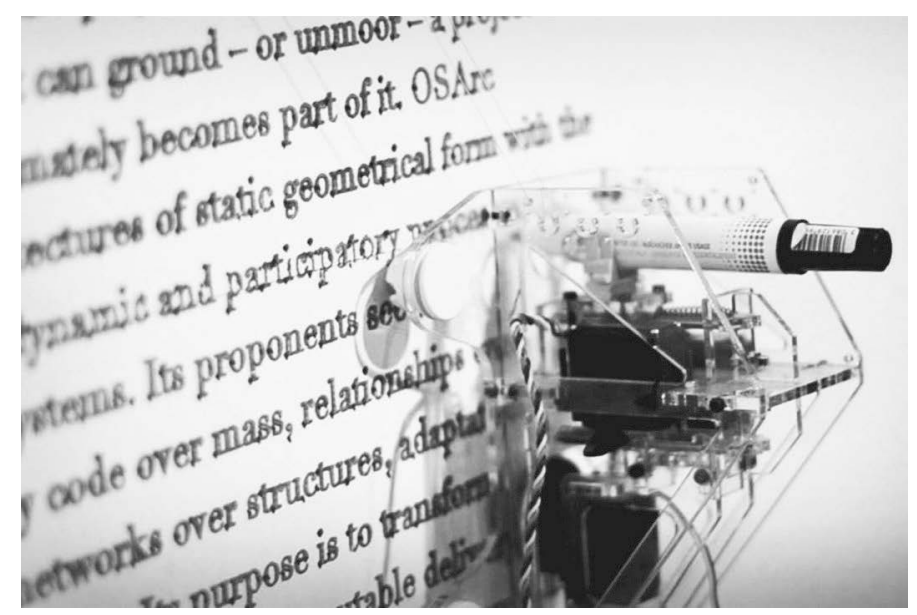

1 Carlo Ratti Associati, Open Source Architecture Manifesto, Installationsansicht Istanbul Biennale 2012

zial entstand eine Konstellation, innerhalb derer dem ,building process“ eine größere Autonomie, und dem Architekten oder der Architektin eine vor allem vermittelnde, ja gar , harmonisierende“ Rolle zugeschrieben wurde:

„Zwischen Le Corbusiers maßgeblicher, das Zeitalter bestimmender Stimme und dem zerstreuten, kollektiven Geplänkel des Internet wird ein Gestalter, der in vernetzte Gemeinschaften verstrickt ist, Harmonie schaffen. Der Architekt wird nicht anonym sein, sondern plural und kompositorisch. Autorschaft wird nicht ausgelöscht, sondern kontextualisiert, indem sie in ein Beziehungsgefüge eingewoben ist. Der neue Architekt situiert sich zwischen Top-Down und Bottom-Up und kanalisiert die rohe Energie des letzteren Prozesses durch die gezielte Rahmensetzung des ersteren. Die Verantwortung des Choral Architect ist weniger auf das Bauen denn auf das Orchestrieren von Prozessen ausgerichtet."3I

Dem Schreiben kommt in einer derart multidirektionalen Kommunikation weniger eine kreative, das poetologische Potenzial der Sprache ausschöpfende, sondern eine den vielstimmigen Diskurs (er-)öffnende und organisierende Rolle zu. Der schreibende oder sich anders sprachlich artikulierende Architekt wird mithin zum Verfasser der ,operating software“32 innerhalb eines Open Source Prozesses, dessen Entwicklung von allen Akteurinnen und 
Akteuren laufend modifiziert und weiterentwickelt werden kann. Es bleibt abzuwarten, inwieweit eine solche vom kategorischen Imperativ der Partizipation und Diskursivität gestiftete ,Vision“33 langfristig das Berufsbild und die kreative Identität heutiger Architektinnen und Architekten prägen wird. Die Vergabe des PritzkerPreises 20 I6 an Alejandro Aravena (der in Open Source Architecture ebenfalls zu Wort kommt ${ }^{34}$ ) und seine Berufung zum Leiter der Architekturbiennale Venedig im gleichen Jahr untermauerten diese Entwicklung jedenfalls; und auch die Pritzker-Jury entschied sich schon im Folgejahr erneut für ein Büro - RCR Arquitectes aus Katalonien -, dessen kollaborativen Ansatz und Orientierung am Gemeinwohl sie in ihrer Begründung ausdrücklich hervorhob. ${ }^{35}$

Architektur und die Sprache des Internets Zur geschriebenen Sprache der Traktate und Manifeste, die jahrhundertelang die Verbreitung architektonischer Ideen gewährleistete, ist in der jüngsten Vergangenheit mit Nachdruck das gesprochene Wort getreten, mittels dessen sich Architektinnen und Architekten über die Kanäle der Sozialen Medien präsentieren: „Architekturkommunikation heißt, sich mit nicht-architektonischen Mitteln eine architektonische Identität zu geben. Oder besser: eine Meta-Identität, die die architekturpraktische, die architekturtheoretische, die Marken- und Unternehmensidentität eines Büros in sich vereint." 36 Der Autor dieser Zeilen, Michael Kuhn, ist Head of Communications des Architekturbüros von Gerkan, Marg und Partner (gmp), dessen Gründer ihrerseits durch rege Vortragstätigkeit, Talks und Interviews an der Hervorbringung der „Meta-Identität“ des Büros teilhaben. Archiviert sind die Aktivitäten auf der gmp-Website, wo in regelmäßigem Abstand Pressemappen und Informationen zu Ausstellungen, Planungsvorhaben und Auszeichnungen veröffentlicht werden. Auf Youtube und Vimeo finden sich zahlreiche Videoclips von Projektvorstellungen oder Diskussionsbeiträgen des Büros. Die in Hamburg gegründete, international tätige Architektensozietät sei hier nur stellvertretend für die Vielzahl an (größeren und kleineren) Architekturbüros genannt, die in ihren Marketingstrategien die gesamte Breite an kommunikativen Möglichkeiten ausschöpfen. Die Sprache der Sozialen Medien ähnelt in diesem Zusammenhang durch ihre informelle Kürze ohnehin eher dem gesprochenen als dem geschriebenen Wort, und auf Kanälen wie Instagram überwiegt zudem die bildliche Kommunikation. Die 
Auswirkungen dieser Ikonisierung auf das gestalterische Denken - in der Architektur, aber auch in anderen Design-Bereichen zeichnen sich unter dem Schlagwort Instagrammability bereits ab. Der schnelle Austausch, die Vermischung textlicher und bildlicher Elemente und eine gewisse ironische Grundhaltung sind weitere Aspekte der digitalen Kommunikation. Im Jahr 2015 unternahm es eine kleine Ausstellung im Art Institute of Chicago zu zeigen, welche Effekte der Einzug von Twitter, Facebook und Co. in die Büros einer jüngeren Architektengeneration hat. Durch den Titel „Chatter. Architecture Talks Back“ wurde die Idee von mehrdirektionalen Gesprächen und fragmentierten Narrativen konnotiert, die durch den permanenten Austausch letztendlich häufig eher zu einer umfangreichen Bild- und Textproduktion denn zum Entwurf konkreter Gebäude führten. ${ }^{37}$ Verbindendes Element der vorgestellten Studios war gleichwohl ein integrativer Blick auf die Baukunst - wiederum wurde den Architektinnen und Architekten eine explizit gesellschafts- und umweltgestaltende Rolle zugesprochen -, sowie ein Denken in Möglichkeiten abseits professioneller Zwänge. Das (inzwischen) bekannteste der seinerzeit an der Ausstellung teilnehmenden Büros ist Bureau Spectacular, dessen Gründer Jimenez Lai 20 I2 eine als Graphic Novel gestaltete Kurzgeschichtensammlung mit dem Titel Citizens of No Place vorlegte. Unterschiedliche Stile aus der Comic-Tradition zitierend, griff sie anhand futuristischer Bild-Text-Erzählungen stadtplanerische und ökologische Probleme und Technologien der Gegenwart und Zukunft auf (und unterschied sich so von Bjarke Ingels' reale Bauprojekte behandelndem Archicomic). ${ }^{38}$ Der aus Taiwan nach Nordamerika übergesiedelte Lai bezieht sich in seiner primär textlich-erzählerischen, weniger konkret baulichen ${ }^{39}$ Praxis auf die sogenannten Paper Architects, deren theoretische Wirkung ihren gebauten ,Output" weit überstrahlt, und agiert insofern weit weniger geschichtsfern, als sein comic-ähnlicher publizistischer und architektonischer Stil vielleicht vermuten lassen. In der folgenden Publikation The Politics of Flatness (2015) ließ er denn auch das architektonische Formenarsenal der Vergangenheit, vor allem der Moderne und Postmoderne, ebenso wie architektonische Darstellungstechniken und -konventionen Revue passieren (Abb. 2). ${ }^{40}$ The Politics of Flatness war Teil eines Meta-Diskurses zur Architekturpraxis, im Zuge dessen Lai dreizehn weitere Architekturbüros eingeladen hatte, sich am kuratorisch-publizis- 


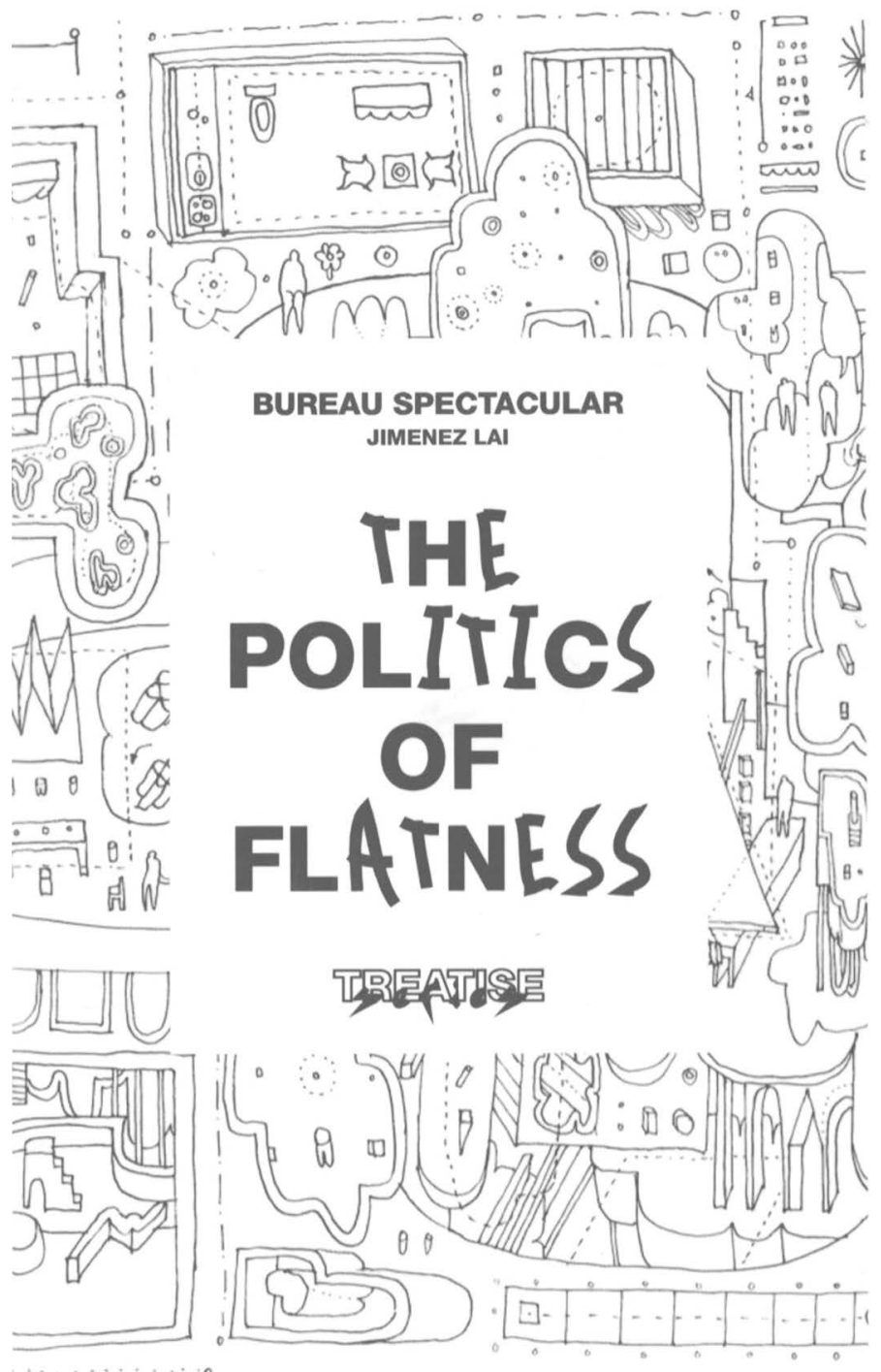

2 Bureau Spectacular / Jimenez Lai, The Politics of Flatness,

Graham Foundation, Chicago, 2015

tischen Projekt Treatise. Why Write Alone? zu beteiligen. ${ }^{4 \mathrm{I}}$ Nach dem Vorbild der von Steven Holl und William Stout I978 etablierten Schriftenreihe Pamphlet Architecture verstand sich Treatise als Sammlung junger Stimmen und individueller Blickpunkte 
- ausdrücklich ohne den Anspruch auf Allgemeingültigkeit, den die Referenz auf die Textform Traktat zunächst suggerieren mochte. Indem die einzelnen Positionen als heterogene Auswahl zusammengestellt und schließlich in einer gemeinsamen Ausstellung präsentiert wurden, organisierte sich das Projekt nach dem Vorbild des kompilierenden Internets, wie auch der Fokus auf Vernetzung sowie die bewusst unfertige Form der Statements die Instantaneität des Internets abbildeten. ${ }^{42} \mathrm{Im}$ übertragenen Sinne ließ sich auch hier die Vielstimmigkeit von Chatter vernehmen, in dem Sinne, dass Heterogenität, Diversität und Unabgeschlossenheit als Qualitäten verstanden wurden, die den architektonischen Diskurs voranzubringen vermögen.

Wohin die Sprache des Internets das gestalterische Denken und die bauliche Praxis letztlich führen wird, bleibt abzuwarten. Dass gleichzeitig das Interesse auch an konsistenter, expliziter Theoriebildung weiterhin vorhanden ist, belegen jedenfalls jüngere Publikationen. ${ }^{43}$ Darüber hinaus weisen aber Projekte wie Chatter oder Treatise mit Nachdruck die geschriebene und gesprochene Sprache als wenn auch oftmals übersehenes oder marginalisiertes, so doch vitales und genuin generatives Medium der Architektur aus, durch das entwurfliche und im weiteren Sinne kulturelle und gesellschaftliche Prozesse in Gang gesetzt und gehalten sowie Handlungspotenziale verteilt werden können.

\section{Anmerkungen}

1 Abrufbar unter: www.pritzkerprize.com/laureates/2016 [11.4.2019].

2 Georg Andreas Böckler, Neues und zuvor nie also eingerichtetes, vollkommenes Seulen-Buch, Frankfurt a. M. 1684, zit. nach: Elisabeth Kieven, „Architekturzeichnung. Akademische Entwicklungen in Rom um 1700", in: Wolfgang Sonne (Hg.), Die Medien der Architektur, Berlin, München 2011, S. 15-31, hier S. 15.

3 Vgl. Susanne Hauser, „Architekturbeschreibung. Über die Sichtbarkeit von Bauten in Texten", in: Sonne (Hg.), Medien der Architektur (Anm. 2), S. 245-253.

4 Homer, Odyssee, 7. Gesang, Vers 84, 132-135, zit. nach: www.gottwein.de/Grie/hom/od07de.php [30.8.2016].

5 Vgl. hierzu mit weiterer Literatur: Barbara Rusch, „François Rabelais, Das höchst schreckliche Leben des großen Gargantua, des Vaters des Pantagruel, 1543", in: Winfried Nerdinger, Hilde Strobl (Hg.), Architektur wie sie im Buche steht. Fiktive Bauten und Städte 
in der Literatur, München 2006, S. 219-222; Erich Köhler, „Die Abtei Thélème und die Einheit des Rabelais'schen Werks", in: August Buck (Hg.), Rabelais, Darmstadt 1973, S. 296-314.

6 Siehe etwa den Forschungsbereich „Bauformen der Imagination" an der Freien Universität Berlin, www.bauformen-der-imagination. de (30.8.2016); Nerdinger, Strobl (Hg.), Architektur wie sie im Buche steht (Anm. 5); Karin Harrasser, Roland Innerhofer (Hg.), Bauformen der Imagination. Ausschnitte einer Kulturgeschichte der architektonischen Phantasie, Wien 2006; Klaske Havik, Urban Literacy. Reading and Writing Architecture, Rotterdam 2014.

7 Hermann Conradi, Adam Mensch, Leipzig 1889, zit. nach: Thomas Althaus, „Von den Stockwerken des Lebens und von der Tücke des Objekts. Friedrich Theodor Vischers Roman Auch Einer und sein Held als Phraseur", in: Barbara Potthast, Alexander Reck (Hg.): Friedrich Theodor Vischer. Leben - Werk - Wirkung, Heidelberg 2007, S. 169-190, hier S. 190.

8 Althaus, „Von den Stockwerken“ (Anm. 7), S. 172.

9 Vgl. Ulrike Steiner, „Umberto Eco, Der Name der Rose, 1980“, in: Nerdinger, Strobl (Hg.), Architektur wie sie im Buche steht (Anm. 5), S. 394-397 mit weiterer Literatur.

10 Vgl. John L. Austin, Zur Theorie der Sprechakte, Stuttgart 1972 (OA 1962); Richard M. Rorty (Hg.), The Linguistic Turn. Recent Essays in Philosophical Method, Chicago 1967.

11 Vgl. Hayden White, Metahistory. Die historische Einbildungskraft im 19. Jahrhundert in Europa, Frankfurt a. M. 1991 (OA 1973).

12 Vgl. Claus Dreyer, „Semiotik und Ästhetik in der Architekturtheorie der sechziger Jahre", in: Susanne Hauser, Daniel Gethmann (Hg.), Kulturtechnik Entwerfen. Praktiken, Konzepte und Medien in Architektur und Design Science, Bielefeld 2009, S. 179-201.

13 Vgl. www.die-poetische-landschaft.de/konzept.html [6.9.2016].

14 Vgl. Jacques Derrida, Peter Eisenman, Chora L Works, New York 1997; siehe hierzu Anthony Vidler, „Nothing to Do with Architecture", Grey Room 21, 2005, S. 112-127.

15 Vgl. Ludwig Jäger, „Medialität”, in: Ekkehard Felder, Andreas Gardt (Hg.), Handbuch Sprache und Wissen, Berlin u.a. 2015, S. 106-122, hier S. 112f., der sich auf Sybille Krämer bezieht.

16 Vgl. Mario Carpo, Architecture in the Age of Printing. Orality, Writing, Typography and Printed Images in Architectural Theory, Cambridge, Mass. 2001. 
17 Anthony Vidler, "From Manifesto to Discourse", in: Craig Buckley (Hg.), After the Manifesto. Writing, Architecture and Media in a New Century, New York 2014, S. 24-39.

18 Ebd., S. 35: „a search for (quasi)-autonomy and new guiding principles that would authorize its role in a newly heterogenenous world."

19 Vgl. Jane Jacobs, "The Miniature Boom", Architectural Forum 5, 1958, S. 106-111; siehe Oliver Elser, „Zur Geschichte des Architekturmodells im 20. Jahrhundert", in: Oliver Elser, Peter Cachola Schmal (Hg.), Das Architekturmodell, Ausst.-Kat. Deutsches Architekturmuseum, Frankfurt a. M. 2012, S. 11-22: hier S. $17 f$.

20 Vidler, "From Manifesto" (Anm. 17), S. 35.

21 Beatriz Colomina, "Manifesto Architecture", in: Buckley (Hg.), After the Manifesto (Anm. 17), S. 40-61: hier S. 42.

22 Ebd., S. 42.

23 Ebd., S. 44; Vidler, "From Manifesto" (Anm. 17), S. 36. Zur Thematik s.a. Christina Anna Kloke, "Manifeste im Wandel”, in: GAM 11, 2015, S. 229-237.

24 Colomina, "Manifesto Architecture” (Anm. 21), S. 59.

25 Rem Koolhaas, Bruce Mau, S, M, L, XL, New York 1995, S. XIX ("chaotic adventure").

26 Ebd.

27 Bjarke Ingels Group (BIG), Yes is More. Ein Archicomic zur Evolution der Architektur, Köln 2010 (OA 2009).

28 Domus 948, Juni 2011, unter: www.domusweb.it/en/ op-ed/2011/06/15/open-source-architecture-osarc-.html [8.9.2016]; von dort wird man verlinkt auf den Wiki-Eintrag: www. en.wikipedia.org/wiki/Open-source_architecture [8.9.2016]; Carlo Ratti, Matthew Claudel (Hg.), Open Source Architecture, London 2015.

29 Der Ausstellungsbeitrag stammte von Walter Nicolino und Carlo Ratti.

30 Beiträger waren u.a. Nicholas Negroponte, John Habraken, Paola Antonelli, Hans Ulrich Obrist.

31 Ratti, Claudel (Hg.), Open Source Architecture (Anm. 28), S. 110f: „Situated between Le Corbusier's authoritative, era-defining voice and the Internet's dispersed collective banter, a designer enmeshed in networked communities will make harmonies. The architect will not be anonymous, but plural and compositional. Authorship will not be erased, but contextualized as it is woven into a relational fabric. The new architect is situated between top-down and 
bottom-up, channeling the raw energy of the latter through the targeted framework of the former. The responsibility of the Choral Architect is less oriented toward object-building than orchestrating process." Übersetzung durch die Autorin.

32 Ebd., S. 112.

33 Siehe die Verlagsankündigung unter: www.thamesandhudson.com/ Open_Source_Architecture/9780500343067 [6.9.2016].

34 Ratti, Claudel (Hg.), Open Source Architecture (Anm. 28), S. 108.

35 Siehe dazu die Ausstellung "The Other Architect", CCA Montreal, 28. Oktober 2015-10. April 2016, die architektonische und städtebauliche Praktiken jenseits des konkreten Bauens vorstellte.

36 Michael Kuhn: [Vorwort], in: Alexander Gutzmer, Architektur und Kommunikation, Bielefeld 2015, S. 7-8, hier S. 7.

37 Karen Kice (Hg.), Chatter. Architecture Talks Back, Yale 2015. Beteiligt waren Bureau Spectacular, Erin Besler, Fake Industries Architectural Agonism, Formlessfinder, John Szot Studio, sowie, in einem weiteren Teil der Ausstellung, Ecosistema Urbano, over, under and pinkcomma, Mimi Zeiger/Neil Donnelly und andere.

38 Jimenez Lai / Bureau Spectacular, Citizens of No Place. An Architectural Graphic Novel, New York 2012.

39 Realisiert wurde etwa der Tower of Twelve Stories als temporäre Architektur auf dem Coachella in Kalifornien (2016) sowie die Installation Flipping Properties in Toronto (2013-14).

40 Jimenez Lai, The Politics of Flatness, Chicago 2015.

41 Ausstellung in der Graham Foundation for Advanced Studies of the Fine Arts, Chicago, 23. Januar-28. März 2015.

42 "We will do it [das Treatise-Projekt, K.N.] now and move on with our lives, leaving its call to be taken up by another generation. The afterlife of this project can be found on the web [...]." Jimenez Lai, "Why Write Alone?", in: Lai, The Politics of Flatness (Anm. 40), Chicago 2015, S. $100 f .$, hier S. 101.

43 Vgl. etwa James Graham (Hg.), 2000+ The Urgencies of Architectural Theory, New York 2015; das Themenheft "Tausendundeine Theorie", Arch+221, 2015; das Themenheft "Archiscripts", GAM 11, 2015. 


\section{Über die Wirkmacht der Linie: Hadids Kalligramme}

Gesteigerte Reflexion der Zeichnung $\mathrm{Zu}$ den nachhaltigsten Prägungen des architektonischen Entwerfens gehört das vereinfachte Idealszenario, wonach der Prozess der Theorie- und Formbildung eine Kette von Modellierungsstufen sei, die vom Großen zum Kleinen, vom städtebaulichen Entwurf zur baukonstruktiven Detailplanung führen. ${ }^{\mathrm{I}}$ Nach diesem Szenario steht am Anfang jeder Modellierungsphase eine architektonische Hypothese mit ihrem je spezifischen Gegenstandsversprechen. Als letzte Modellierungsstufe am Ende der Kette liegt das konkrete Bauwerk im Eins-zu-eins-Maßstab vor. Jede Stufe wird in maßstabsgetreuen Zeichnungen und Modellen entwickelt, die ihre Qualität aus der Entsprechung zum späteren Bauwerk gewinnen. In jeder Phase hat der Architekt oder die Architektin die Möglichkeit, das bisher Modellierte durch Anschauung zu überprüfen, weiter auszuarbeiten oder zu verwerfen. Auf diese Weise wird der Entwurf von einer Stufe auf die nächste überführt.

Das computerbasierte Modellieren scheint diese ideale Abfolge entwerferischer Operationen zu unterbrechen. Jörg H. Gleiter spricht hierbei von einem „Abreißen der Modellierungskette“. ${ }^{2}$ Am Computer entwickelt der Architekt oder die Architektin den Entwurf weniger in aufeinander aufbauenden Stufen als vielmehr in einer einzigen Stufe, die theoretisch alle anderen Stufen beinhaltet. Die erdachte Architektur wird nicht in abstrahierenden, voneinander getrennten Zeichnungen dargestellt, sondern in einem einzigen, zweidimensional wiedergegebenen 3D-Modell visualisiert. Digitale Prozessketten heben die klassische Trennung zwischen intellektuellem Entwurfsakt und materieller Ausführung auf, die Leon Battista Alberti in seinen Zehn Büchern über die 
Baukunst verbindlich für die Architektur gefordert hatte - so lautet die zentrale These von Mario Carpo, weshalb er diesen Wendepunkt auch als „Umkehrung des albertianischen Paradigmas“ charakterisierte. ${ }^{3}$ Während im Analogen die Entwurfszeichnungen zunächst in Ausführungspläne und anschließend von den am Bau beteiligten Gewerken in Werkstattpläne transformiert werden, sind sie im Digitalen von Anfang an auf das engste mit der Bauplanung und -ausführung verknüpft. Die Produktionstechnologien greifen unmittelbar in die Entwurfsverfahren ein und nehmen Einfluss auf den Gegenstand des Entwerfens: Bei dem file to factory genannten Modellierungsverfahren werden geometrische und technologische Informationen in einem Datenmodell zusammengefasst, das unter Zuhilfenahme computergesteuerter Fertigungsmaschinen (unter anderem von 3D-Druckern) in ein physisches Modell oder ein Bauteil umgesetzt wird.

Angesichts dieser fundamentalen Veränderungen der Entwurfsprozesse wurden in den vergangenen Jahren drastische Krisenszenarien vom Bedeutungsverlust des manuellen Zeichnens und vom buchstäblichen Verschwinden der Grundlagen der architektonischen Praxis beschworen, wohingegen die Betrachtung der Potenziale des Entwerfens am Computer unterbelichtet blieben. Löst man sich von der These einer Krisenerscheinung und lässt sich mehr auf die Eigenheiten des computerbasierten Entwerfens ein, so eröffnen sich neue Sichtweisen auf die produktiven Wirkungen digitaler Zeichnungen. Das Zeichnen zeigt sich dann als probeweises und systematisches Durchspielen verschiedener Variationen von Denk- und Raummodellen. ${ }^{4}$ Es zeigt sich als bewusstes Erzeugen von , nicht-lesbaren' Zeichnungen und Formen, um durch diese Irritation zu noch unbekannten Entwurfsideen zu gelangen; es zeigt sich aber auch in der Erkundung neuer Systeme oder Figurationen, denen eine generative Funktion zukommt.

Digitale Zeichnungen sind insofern von übergreifender Bedeutung, als sie tradierte Erwartungen an das, was eine Zeichnung in der Architektur sei, in Frage stellen. Durch die interaktive Verknüpfung von Entwurf und Ausführung verkörpern sie nicht nur ein dienendes Verhältnis der Zeichnung zum Gezeichneten, sondern zeigen ein komplexes Geflecht von Effekten und Rückwirkungen auf. Das Modellieren von Materialeigenschaften und Kraftfeldern im digitalen 3D-Raum, wie es seit den I990er Jahren in der experimentellen Architekturpraxis erprobt wird, verlässt die Vorstellung, 
dass die Zeichnung lediglich ein abstraktes, auf das Konzept der Repräsentation verpflichtetes Schema sei. Vielmehr erweisen sich Zeichnungen als operative Bildsysteme, die wesentlich durch ihr Verhältnis zu Materie und Stoff bestimmt sind. Ebenso wird das vorherrschende Vorurteil aufgehoben, dass Zeichnungen, insbesondere Perspektiven, infolge ihrer statischen Verfasstheit nicht in der Lage seien, dynamische Prozesse wiederzugeben. Unter Zuhilfenahme von digitalen 3D-Animationstechniken ist die Zeichnung weniger Repräsentant eines Formzustandes, der auf einen festen Blickpunkt des Betrachters bezogen ist, als vielmehr Träger von allansichtigen Formbewegungen.

Die neuen Verfahren digitaler Produktion greifen aber nicht nur in die gegenwärtige Entwurfspraxis der Architektur und deren theoretische Grundlagen ein, sondern motivieren auch zur Rekonzeptualisierung tradierter Praktiken und Medien. Im Lichte digitaler Modellierungstechniken werden bisher wenig wahrgenommene Aspekte der Handzeichnung sichtbar und neue Perspektiven auf manuelle Zeichenpraktiken erschlossen. Das computerbasierte Entwerfen ändert den Blick auf die Geschichte der architektonischen wie künstlerischen Zeichnung und lässt bisher nicht erkannte Aspekte des Digitalen im Vergangenen aufscheinen.

Im Folgenden werden daher experimentelle Zeichnungen der digitalen Architekturavantgarde in den Blick genommen, die zwar mit der Hand entwickelt wurden, aber vom Digitalen , infiziert' sind. Exemplarische Beispiele hierfür sind die Zeichnungen und Skizzen der britisch-irakischen Architektin Zaha Hadid ( I95020I6), die im digital turn am Ende des 20. Jahrhunderts entstanden sind. Ausgehend von der formalen Analyse dieser zeichnerischen Experimente werden Traditionen und Strategien freigelegt, die den Einsatz und die Interpretation digitaler Zeichenverfahren bis heute motivieren und prägen. Zugleich werden die historischen Vorbilder aus der Perspektive des Digitalen neu beleuchtet. In den Fokus der Auseinandersetzung geraten vor allem Linienpraktiken und -konzepte der europäischen Kunst zwischen dem I5. und frühen 20. Jahrhundert. Hierbei gilt das Augenmerk Konzepten, welche die Idee der Umriss- und Konturlinie durch Vorstellungen von Kraft, Rhythmus und Ausdruck ersetzten und den Eigenlauf der Linie betonten. 
Überschießende Wirkungen Die Frage nach den Potenzialen digitaler und vor-digitaler Zeichnungen greift jene bild- und modelltheoretischen Überlegungen auf, die in den vergangenen Jahren vor allem in der Wissenschafts- und Kunstgeschichte entwickelt wurden. Zeichnungen und Modelle erschöpfen sich beispielsweise für den Mathematiker und Wissenschaftstheoretiker Bernd Mahr sowie für die Kunsthistoriker Horst Bredekamp und Reinhard Wendler nicht nur darin, dass sie Abbilder ,von etwas“ oder Vorbilder ,für etwas‘ sind. ${ }^{5}$ Sie fungieren ebenso als Gebilde, die in ihrer Form und Wirkmacht so eigenständig sein können, dass sie selbst für den Bezugsgegenstand gehalten werden: Zeichnungen und Modelle ,,sind [...] auch an sich. Sie haben eine eigene Brillanz, Ästhetik und setzen sich sehr oft an die Stelle dessen, was sie modellieren." ${ }^{6}$ Sie entwickeln einen Eigenlauf, indem sie zu verschiedenen Lesarten und Handlungen motivieren, die wiederum konstitutiv auf das einwirken, was sie vermitteln. Im Akt des Zeichnens ereignen sich, so Bredekamps weiterführende Diagnose, unvorhersehbare Wirkungen, die der Intention des Zeichnenden und der richtungsweisenden Kraft der Vorbilder entzogen sind. ${ }^{7}$ Diese Effekte ergeben sich aus der Materialität des Zeichenwerkzeugs und des Zeichnungsträgers, aber auch aus dem Zusammenspiel von Zeichnungsgegenstand, -ziel und -kontext. Zeichnungen produzieren somit einen, über ihre engere Bestimmung hinausgehenden, die Bereitschaft zum Handeln und zum Denken stimulierenden Überschuß“ an Sinn, der meist aus einem Mangel an Bestimmtheit der Zeichnungen entsteht. ${ }^{8}$ Dieser imaginativ weitertreibende Überschuss macht eine vollständige Kontrolle von Zeichnungen und Zeichnungsprozessen unmöglich. Ein Zeichnungsprozess verläuft daher weniger linear als iterativ, das heißt, er beginnt selten mit einer Idee und endet mit der Umsetzung einer Zeichnung in ein materielles Objekt. Eher stößt jede Begegnung mit einer Zeichnung eine Wirkungsfolge an, in der weitere aufeinander bezogene Zeichnungen erzeugt werden.

Der Ansatz, Zeichnungen nicht nur als Ausdruck und Widerschein von bestehenden oder zukünftigen Sachverhalten, sondern auch als autonome, wirkmächtige Akteure zu begreifen, die Denkund Entwurfsprozesse auszulösen in der Lage sind, gründet aber auch wesentlich auf dem Begriff des Diagrammatischen, wie er seit den I990er Jahren in Architektur und Philosophie neu verhandelt wird. So gilt etwa dem US-amerikanischen Architekturtheore- 
tiker Robert Somol die strukturelle Eigenlogik der Zeichnung als Charakteristikum des Diagramms. In seinem programmatischen Aufsatz Dummy Text, or The Diagrammatic Basis of Contemporary Architecture von 1999 zeigte er, wie sich die Entwurfszeichnung infolge neuer Bildgebungstechniken in ein Entwurfsdiagramm verwandelt hat. 9 Dabei habe sich die Zeichnung aus ihrer Hilfs- und Vorläuferfunktion befreit und sich zum Impulsgeber und Reflexionsinstrument in der Architektur entwickelt. Die Zeichnung, wie sie Somol definiert, ist nicht mehr ein Medium der reinen Naturnachahmung, sondern ein Instrument zur Erforschung von abstrakten Strukturen und Beziehungsgefügen. Ähnlich sieht die deutsche Philosophin Sybille Krämer im Diagramm weniger ein Visualisierungsinstrument als vielmehr ein Denkwerkzeug: „Diagramme zeigen nicht einfach nur ,Gegenstände“, sondern Relationen innerhalb von Begriffs- und Wissensfeldern. Dadurch eröffnen Diagramme nicht nur einen Darstellungsraum, sondern auch einen Raum des Experimentierens, Explorierens und Operierens mit oftmals abstrakten [...] Sachverhalten". ${ }^{\text {Io }}$ Die zweidimensionale Visualisierung von Relationen, die sowohl Zeichnung wie Schrift verbindet, wird von Krämer zu einem epistemischen Prozess erklärt, der im Kern von Vorläufigkeit und Unsicherheit durchzogen ist und sich auf noch unbekannte Dinge mit verschwommenen Grenzen richtet.

Exemplarisch lässt sich ein solch ergebnisoffener Erkenntnisund Entwicklungsprozess an den zeichnerischen Entwürfen von Zaha Hadid nachvollziehen. Im Zuge des digital turn widmete sich die Architektin der freien Linie und spontanen Aufzeichnungen, die sich im Spannungsfeld von Architektur und Kalligrafie bewegen. Mit Blick darauf wird der Frage nachgegangen, in welcher Weise Zeichnen und Schreiben als Verfahren des Entwurfs zusammentreten, und wie die Wechselwirkungen der verschiedenen Aufzeichnungsverfahren die Gestaltung und Auswahl architektonischer Motive bestimmen.

Hybride aus Bild und Sprache Bekannt ist, dass Hadid ihre Entwürfe der I980er und frühen I990er Jahre in großformatigen Acrylgemälden entwickelte, in denen sie nicht primär Repräsentationen von Gebäudeentwürfen sah, sondern vielmehr Möglichkeiten zur Erkundung komplexer Raumanordnungen: In den Gemälden verschränkte sie isometrische und perspektivische 
Formen so virtuos ineinander, dass der dargestellte Raum radikal verzerrt wurde. ${ }^{\text {I I }}$ Ebenso geläufig ist, dass der Einsatz des Computers in Hadids Büro entscheidend dazu beigetragen hat, dass ihre komplexen Raumsphären baulich umgesetzt werden konnten. Weniger bekannt ist hingegen, dass Hadid unentwegt kritzelte, notierte und skizzierte. Angesichts ihrer schnellen Strichführung und ihrer niemals korrigierten Linien lässt sich vermuten, dass die Skizze für sie weniger eine Ergänzung als eine notwendige Bedingung ihrer architektonischen Produktion darstellte. Auch für das computerbasierte Entwerfen, das ihr Mitarbeiter und späterer Büropartner Patrik Schumacher vorantrieb, waren Hadids Skizzen eine konzeptionelle Voraussetzung. Die Herausforderung in der „Optimierung von technischen Umsetzungsmöglichkeiten“ bestand darin, so Schumacher, „Handskizzen, [...] in denen alle Linien gekrümmt sind, wörtlich zu nehmen". ${ }^{\text {I2 }}$

Hadid brachte ihre Ideen durch ein abstrahierendes Zeichnen hervor, mit dem sie komplexe räumliche Strukturen auf Linienkürzel reduzierte. So erhielt etwa ein Opernhaus für die chinesische Stadt Guangzhou in der zeichnerischen Verkürzung die Gestalt eines Linienknotens mit einem Schweif (Abb. I). Hadid hatte in der Vergangenheit wiederholt ihr Interesse an arabischer Kalligrafie bekundet und dessen ästhetisches wie operatives Potenzial betont. In Projekterläuterungen und Interviews verwendete sie häufig den Begriff der Kalligrafie, um die räumlichen Bewegungen und Muster ihrer Architektur zu beschreiben. ${ }^{13}$ Dabei ließ sie offen, wie die Kalligrafie ihre Projekte konkret beeinflusst hat. Betrachtet man Hadids Skizzen aus der Perspektive der Kalligrafie, so fällt auf, dass sich ihre Linienkürzel assoziativ auf tradierte arabische Schriftfiguren beziehen lassen. Die Skizze für das Opernhaus in Guangzhou erinnert an die traditionelle Form einer Tuğra, die noch heute ein beliebtes Motiv für Wandschmuck, aber auch für Tätowierungen darstellt (Abb. 2). Eine Tuğra mit ihren typischen visuellen Elementen sere (Handfläche), beyze (Ei), tŭ̆ (Rossschweif), zülfe (Locken) und hançer (Dolch) ist ein kalligrafisches Monogramm, das ursprünglich osmanische Sultane im i6. Jahrhundert als imperiales Signum verwendeten. Unfreiwillig ironisch mutet bei diesem Bildvergleich an, dass das 20 Io fertiggestellte Opernhaus nicht nur als das größte Musiktheater Südchinas gilt, sondern auch der chinesischen Führungselite als neues Herrschaftszeichen dient. Aber es wäre eine Verkürzung zu behaupten, dass 

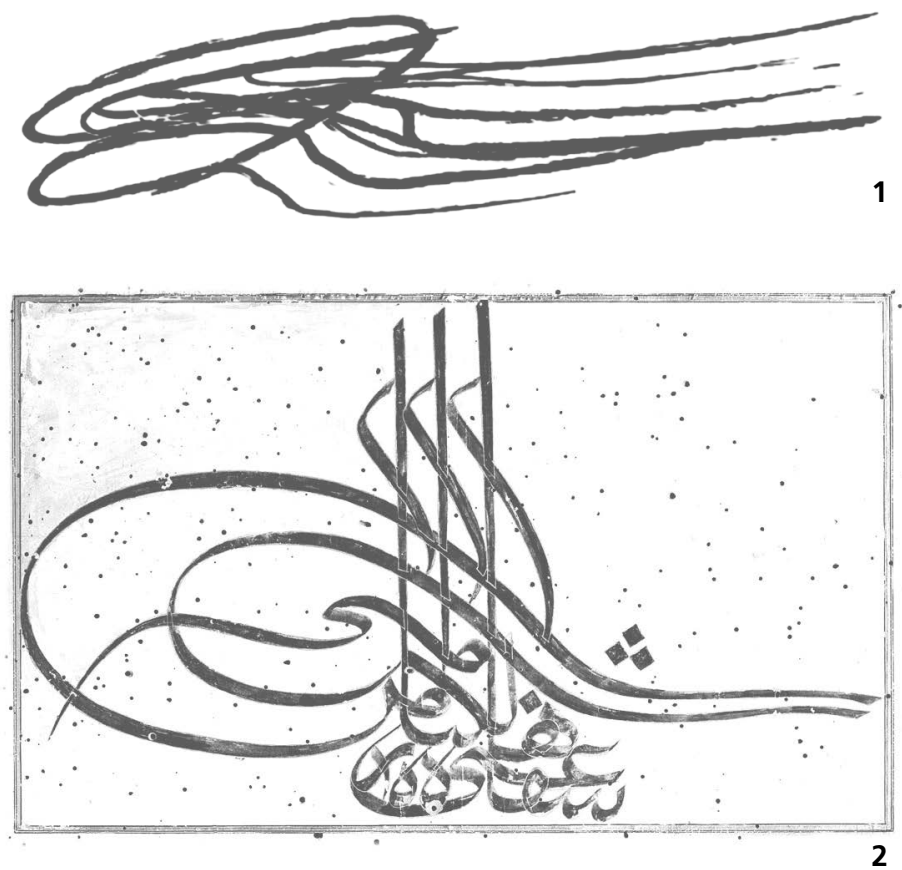

1 Zaha Hadid, Guangzhou Opera House, Guangzhou, China, 2003-2010 (Skizze)

2 Inschrift in Tuğra-Form, Mittlerer Osten, 17. bis 18. Jahrhundert (Tinte auf Papier, aufgezogen auf Holz)

Hadids Entwürfe vor allem auf kalligrafischen und architektonischen Vorbildern der islamischen Kultur beruhen würden. Vielmehr bezeugen sie ein komplexes und vielschichtiges Geflecht von zeichnerischen Einflüssen aus westlicher Moderne und östlicher Tradition, die über Kontexte und Disziplingrenzen hinweg ineinander verschlungen werden.

Draw a distinction In zahlreichen Skizzen zeichnete Hadid nur eine einzige Linie oder zwei Varianten einer Linie, so als würde der erste Strich, der das weiße Papier markiert, die entstehende Architektur vollständig informieren. Wie ein offenkundiges Zitat des englischen Mathematikers George Spencer-Brown muten ihre 

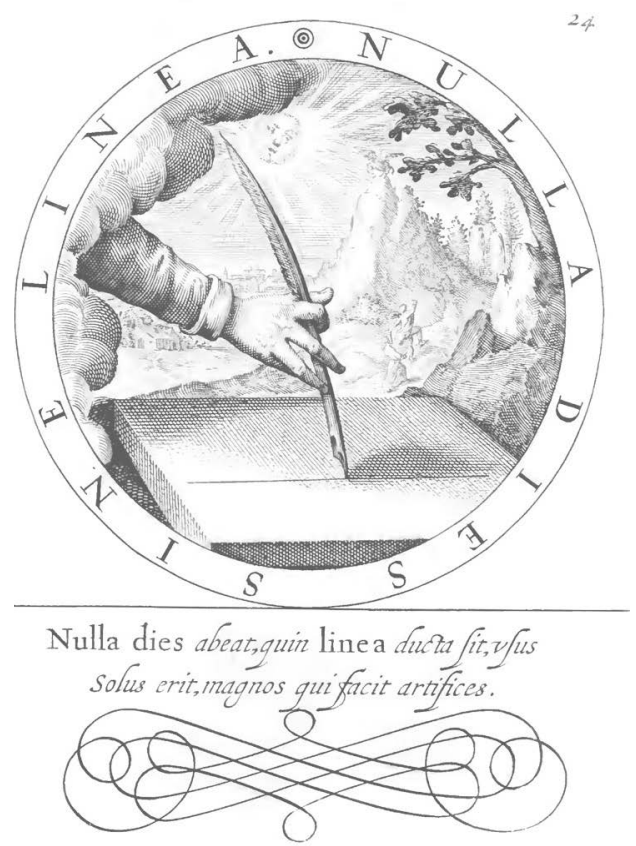

3 Gabriel Rollenhagen, Nulla dies sine linea, 1611

Zeichnungen der ersten Linie an, die den weißen Papiergrund in zwei Bereiche teilt. ${ }^{I 4}$ Mit der Anweisung „Draw a distinction!“ erklärte Spencer-Brown in seinen Laws of Form von I969 den Akt des Unterscheidens und Bezeichnens zur grundlegenden Operation formaler Kalküle der Logik und der Mathematik. ${ }^{15}$ Mit dem ersten Strich wird dort, wo nichts als ungetrennte Potenzialität war, eine Unterscheidung getroffen und ein Raum bezeichnet. ${ }^{16}$ Für den Zeichnenden stellt daher die Setzung durch den ersten Strich das größte Risiko dar. Von ihm hängen alle weiteren Striche und damit das Gelingen oder Misslingen der Zeichnung ab.

Mit dem Eintrag der ersten Linie auf eine weiße Fläche zitierte Hadid neben einem formalen Kalkül auch eine der ältesten Metaphern des schöpferischen Prozesses. Emblematische Werke und zahlreiche Malereitraktate, wie etwa das I6 I in Köln gedruckte Buch Nucleus Emblematum von Gabriel Rollenhagen oder die I633 in Madrid publizierten Diálogos de la Pintura von Vicente Carducho, zeigen den Ansatz zu diesem entscheidenden ersten Strich, der die Möglichkeiten aller Formen in sich birgt (Abb. 3). 

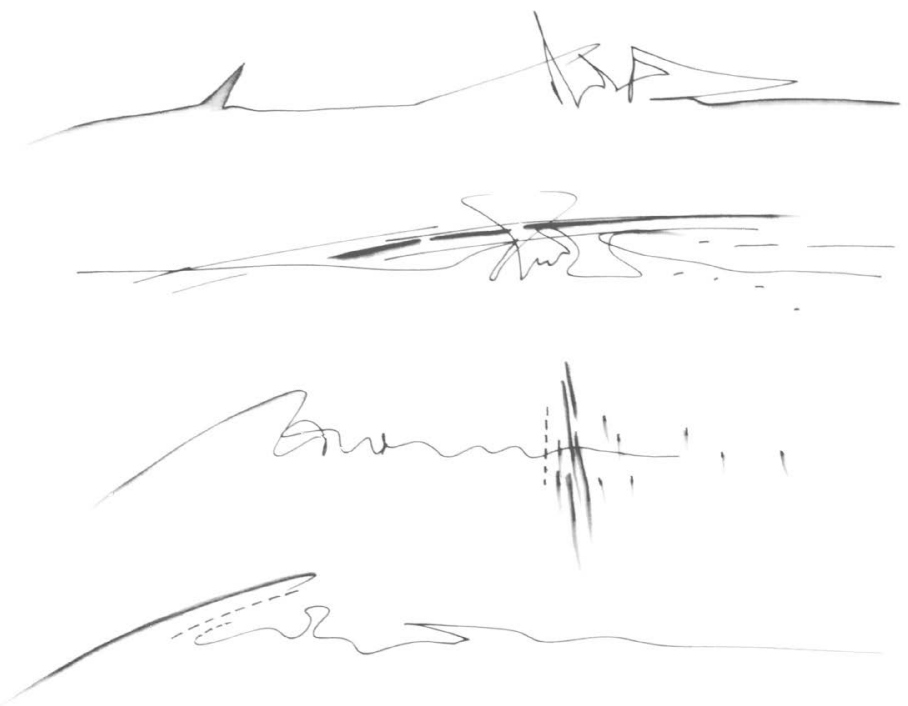

4 Zaha Hadid, Neuer Zollhof 3, MedienHafen, Düsseldorf, 1989-1993 (Skizzen)

Auf beiden Stichen sieht der Betrachter eine Feder beziehungsweise einen Pinsel und eine weiße Leinwand, die nur mit einem schwarzen Strich markiert ist. Auf dem Schlussbild des Traktats von Carducho scheint der Strich sogar nur eine Schattenlinie des Pinsels zu sein, als ob nahgelegt werden soll, dass die Malerei aus der Wiedergabe von Schatten entstanden sei, die sie selbst erzeugt. ${ }^{17}$

In immer neuen Versionen, welche die entscheidenden Linien zeichenhaft isolieren, hat Hadid die Spannung zwischen dem weißen Blatt, dem ersten Strich und den folgenden Linienzügen aufgebaut. So zeigen zwei Skizzen des Feuerwehrhauses für das Schweizer Unternehmen Vitra die initiale Linie programmatisch als gekrümmte Linie. ${ }^{18}$ Zeichnungen des Neuen Zollhofs 3 in Düsseldorf weisen den ersten Strich als eine lange Horizontallinie aus, die durch eine fortlaufende Führung und durch schwungvolle Ablenkungen nach oben und unten charakterisiert ist (Abb. 4). Dieser durchgehende Linienzug, der entlang einer Grundlinie organisiert ist, weist Ähnlichkeiten mit der Linienführung arabischer 
Handschriften auf (Abb. 5). Alle arabischen Schrifttypen sind Kursivschriften, bei denen die meisten Buchstaben eines Wortes verbunden sind. Im Unterschied zur lateinischen Schrift gibt es keine Versalien und keine Blockschrift, die den Linienfluss unterbrechen. Die zu einer Linie verbundenen Buchstaben werden horizontal von rechts nach links geschrieben, wobei sie auf eine imaginäre Standlinie bezogen werden.

Die Verweise auf den ersten Strich der Malerei und die kontinuierliche Linie der Handschrift könnten dazu verleiten, Hadids Zeichnungen als Darstellungen zu deuten, die vom Zweck der Architektur entbunden seien. Bei ihren Skizzen handelt es sich jedoch keineswegs um Ausdrucksformen künstlerischer Freiheit, sondern um grafische Anordnungen, die einerseits von den bedingenden Faktoren des Geländes, der Statik und der Mittel der Architektur bestimmt sind, und anderseits auf Konstruktionen zielen, welche die fixierenden Momente dieser Einflussfaktoren zu überwinden suchen. Für Hadid war es vor allem die bedingende Beziehung zwischen Architektur und Erdboden, die sie neu verhandeln wollte. Bereits in ihrem ersten bedeutenden Entwurf des Peak Leisure Club in Hongkong, mit dem sie in den I980er Jahren internationales Aufsehen erregte, setzte sie dem rechten Winkel die Schräge entgegen und forderte die Abkehr vom lotrechten Stand der Architektur: „There are 360 degrees, so why stick to one?“19 Im Prozess der zeichnerischen Vermittlung zwischen Gebäude und Grund zerlegte sie die architektonische Form in unterschiedlich schräge Linien. Auf diese Weise entstanden reduzierte, abstrakte Zeichen, die aber keineswegs vom architektonischen Gehalt entkoppelt waren. Vielmehr wurde die schräge Linie als geeignete Ausdrucksform für die Überwindung der Schwerkraft angesehen. Eine ähnliche Bedeutung sollte wenige Jahre später die Schlangenlinie erlangen.

Die aktive Linie Die schlängelnde Linie durchzieht Hadids gesamtes zeichnerisches Euvre und findet sich auch schon in den frühen konstruktivistisch inspirierten Zeichnungen für den Peak Leisure $C l u b$ in Hongkong. ${ }^{20}$ Als alleinige Form tritt sie aber erst in ihren Entwürfen am Ende des 20. Jahrhunderts auf, was offenkundig mit der Einführung des Computers in Hadids Büro zusammenhängt. Der Zuwachs an Rechenleistung und die Entwicklung von 3D-Grafikprogrammen zur Bearbeitung topologischer Geometrien 


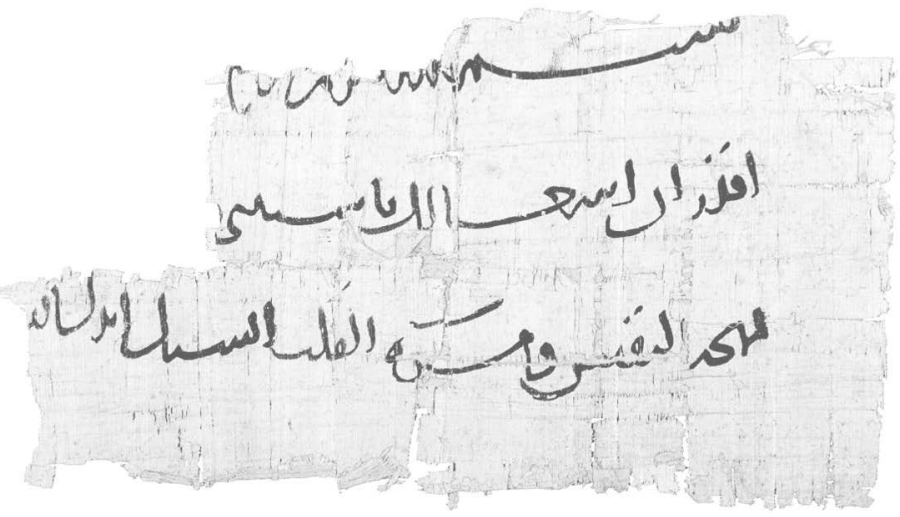

5 Papyrusfragment aus Ägypten, 9. bis 10. Jahrhundert

ließen ihre Architekturformen zunehmend geschmeidiger werden. Obgleich Hadid selbst nicht am Bildschirm entwarf (,I don't use the computer. I do sketches, very quickly, often more than Ioo on the same formal research" ${ }^{\text {"2I }}$ ), begann sich ihre Architektur unter dem Einfluss des computerbasierten Modellierens zu verändern. Die scharfen Winkel der frühen Entwürfe, die von den Zeichnungen des russischen Künstlers Kazimir Malevich (I878-I935) inspiriert waren, wichen zugunsten von biomorphen Formen.

Aber die Wirkung des Digitalen ging noch weiter: Es war nicht nur das Formenvokabular, das sich durch den Einsatz avancierter 3D-Modellierungsprogramme veränderte, sondern auch die Raumvorstellung und das Geometrieverständnis. Während klassische Entwurfszeichnungen, die herkömmlichen Repräsentationskonventionen folgen, vor allem auf der euklidischen und projektiven Geometrie beruhen, basieren digitale Zeichnungen auf algebraischen und arithmetischen Operationen. ${ }^{22}$ Objekte werden dabei nicht, wie beim tradierten Zeichnen, aus Linien und Flächen konstruiert, um den leeren Raum zu füllen, der sich im Koordinatensystem aufspannt. Vielmehr wird eine algebraische Form aus dem Punkt und seiner Trajektorie, das heißt seiner Entwicklungsbahn in der Zeit, generiert. Wenn architektonische Formen zu Punktmassen und Bündeln von Trajektorien werden, lässt sich die Relation zwischen diesen Punkten ebenso wie der Entwicklungsverlauf der Trajektorien immer wieder neu bestimmen. Parameterbasierte 


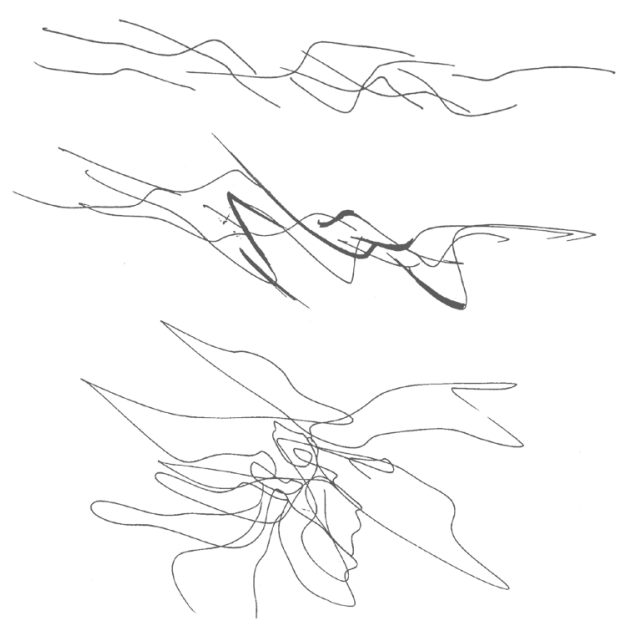

Computerprogramme verfügen über ein hoch entwickeltes System von mathematischen Funktionen, wodurch sie in der Lage sind, auf Basis von Kraftfeldern dynamische Veränderungen der Punkte und Trajektorien zu erzielen. Dabei wird die generierte Linienfigur als ein System aus Elementen verstanden, auf die Kräfte einwirken.

Mit diesem Begriff der digitalen Linie als dynamisches System lassen sich auch die Handskizzen beschreiben, die Hadid seit den I990er Jahren entwarf: Punkte werden durch die Kraft der zeichnenden Hand und des schwingenden Armes in eine freie Bewegung versetzt und geraten zu kontinuierlichen Linien (Abb. 6). Diese Bewegungslinien wandeln sich von einer kräftigen Verdickung bis zu einer sich verdünnenden Auflösung. Sie sind an keiner Stelle korrigiert, und der glatte Durchzug der Linien verdeutlicht die konstante Geschwindigkeit, mit der sie gezeichnet wurden.

Es ist Hadids Verdienst, dass im Lichte ihrer digital bewegten Skizzen Aspekte vergangener Bildpraktiken sichtbar werden, von denen sich Traditionslinien zur Praxis der Computeravantgarde ziehen lassen. So reflektieren ihre linearen Schwingungen nicht nur die neuen Möglichkeiten digitaler Modellierungstechniken, sondern sie stehen zugleich in einer lange zurückreichenden Kette von Versuchen, eine Bildformel für die Bewegungen der Natur und des schöpferischen Denkens zu gewinnen. ${ }^{23}$ Die fortwährende Bewegung der Schlangenlinie als Metapher für eine Vielzahl von Verwandlungsmöglichkeiten und Ambivalenzen lässt sich bis 


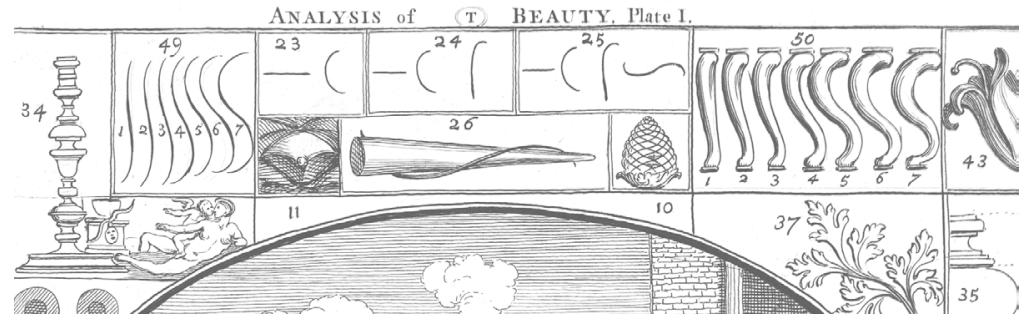

6 Zaha Hadid, Erweiterung des Ordrupgaard Museums, Kopenhagen 2001-2005 (Skizzen)

7 William Hogarth, Tafel 1 zur Analysis of Beauty, 1753 (Radierung, Stich)

auf Leon Battista Alberti zurückverfolgen. Er verglich in seinem Traktat De pictura von I435 die Bewegungen von Haaren mit denen von Flammen und Schlangen. ${ }^{24}$ Albrecht Dürer hat in seiner Schrift Unterweysung der Messung von I525 neben den euklidischen Grundelementen - der Geraden und der Kreislinie - die S-förmige „Schlangenlinie“ als konstitutive Linie hinzugefügt. ${ }^{25}$ Für ihn verkörperte sie die unendliche Vielgestaltigkeit, die Metamorphose, die Bewegung, die nicht beherrschbare Wandel- und Deutbarkeit: „Aber die Schlangenlinie ist unendlich zu verändern; daraus man wunderliche Ding mag machen [...]. “26

Im Jahre I753 fixierte William Hogarth die schlangenförmige Mannigfaltigkeit (variety) als Zeichen der Summe aller Bewegungs- und Darstellungsformen in einem Prisma. ${ }^{27}$ Die erste Abbildungstafel seines berühmten Traktates Analysis of Beauty zeigt in der oberen Bildleiste, wie sich aus der Fügung einer Geraden und einer Kurve eine Schlangenlinie ergibt (Abb. 7). Sie ist das Ergebnis einer systematischen Kombination aus geometrischen Grundelementen, die ihrerseits eine Vielfalt von Variationen erzeugt als abstrakte Formen und als konkrete Erscheinungen der sinnlich wahrnehmbaren Welt: So wiederholen sich in der oberen Bildleiste sieben unterschiedlich gestauchte S-Linien in sieben verschieden geformten Stuhlbeinen. Die Schlangenlinie ist für Hogarth eine universale Strukturform; sie macht unsichtbare Strukturen hinter den Erscheinungen sichtbar. 

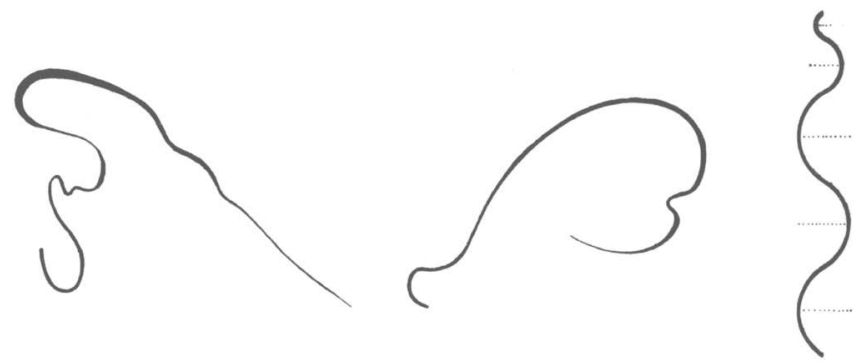

8 Wassily Kandinsky, Frei- und geometrisch-wellenartige Linien, 1926

9 Hermann Finsterlin, Skizzenblatt, 1910 (Bleistift, Kreide auf Transparentpapier)

Im frühen 20. Jahrhundert war es vor allem Paul Klee, der die Schlangenlinie in den höchsten Rang motorischer Energie erhob und damit das Konzept der digitalen Linie gedanklich vorwegnahm. In seinem Pädagogischen Skizzenbuch von I925 definierte er sie als eine umherschweifende Form, die sich aus der raumzeitlichen Verschiebung eines Punktes entwickelt: „Eine aktive Linie, die sich frei ergeht, ein Spaziergang um seiner selbst willen, ohne Ziel. Das agens ist ein Punkt, der sich verschiebt. "28 Ohne diese Annahme wäre auch Gilles Deleuzes und Félix Guattaris Vorstellung von der „Linie des Werdens“ kaum denkbar gewesen: „Geschwindigkeit verwandelt den Punkt in eine Linie!“ - so endet ihr Text Rhizome von I976, in dem sie ein postmodernes Modell flexibler Wissensorganisation beschreiben. ${ }^{29}$

Auch Wassily Kandinsky, der neben Kazimir Malevich als zentraler Anreger von Hadid gelten kann, untersuchte die Linie im Spannungsfeld von Spurbildung und Konstruktion. ${ }^{30}$ In seiner Schrift Punkt und Linie zu Fläche von I926 definierte er die Schlangenlinie als energiegeladene Möglichkeitsform par excellence, die eine konstruktive Aktivität entfaltet (Abb. 8). Vorbildhaft für Hadids Zeichnungen dürfte auch Kandinskys programmatische Auseinandersetzung mit Bildform und Schriftzeichen gewesen sein. Der Künstler war I904 nach Tunis gereist und hatte dort neben typisch orientalischen Motiven wie den Faltenwurf von 


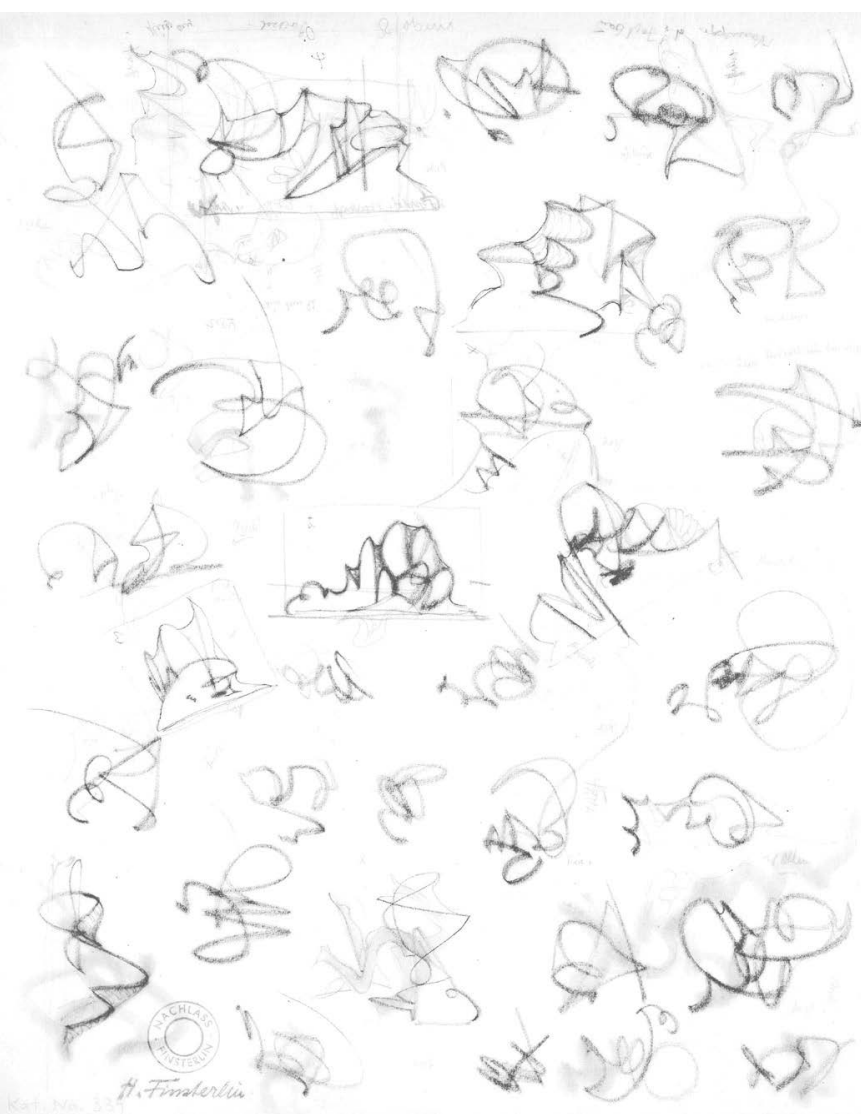

Gewändern auch arabische Kalligrafie studiert. ${ }^{31}$ In seinem frühen Text Über die Formfrage von I9I2 bestimmte er den Buchstaben als ein Gefüge ,aus einzelnen, so oder anders gebogenen Linien“, das nicht nur als abstraktes sprachliches Zeichen, sondern auch als konkretes synästhetisches Bild - jenseits einer beschreibenden, symbolischen Funktion - wahrgenommen werden könne: „Wenn der Leser [...] einen Buchstaben [...] nicht als ein gewohntes Zeichen eines Teiles eines Wortes [anschaut], sondern erst als Ding, so sieht er in diesem Buchstaben ausser der praktisch-zweckmäßig vom Menschen geschaffenen abstrakten Form [...] noch eine körperliche Form, die ganz selbständig einen bestimmten äusseren und inneren Eindruck macht [...], einen Klang. "32 Von hier ausge- 
hend lässt sich die gebogene Linie als körperliche Ausdrucksform bestimmen, die Zeichnung und Schrift gleichermaßen prägt und insofern synthetisierend wirkt.

Eine neue architektonische Grammatik versprachen auch die kalligrafisch anmutenden Linienkürzel, die der deutsche Architekt Hermann Finsterlin wie auf einer Silbentafel aufreihte (Abb. 9). Doch statt eines stabilen Ordnungsgefüges, wie man es bei einer Grammatik erwarten würde, entwickelte Finsterlin ein undurchschaubares Dickicht fortlaufend rotierender und sich kreuzender Linien in Szene. In zahlreichen Versionen skizzierte er verschlungene Liniengebilde, die Ansätze zu neuen kontinuierlichen Räumen und Flächen offenbarten.

Auch Hadid überträgt die S-Linie in ein organisches Kontinuum. In einer Modellskizze des Gebäudes Pierres Vives aus dem Jahr 2002 treibt sie die Form mit immer neuen Kurven kreisend in die Tiefe (Abb. Io). Die Tiefendimension entsteht dadurch, dass die Linienstärke zu- und abnimmt, aber ebenso dadurch, dass die Linie ohne Unterbrechung gezeichnet wird. In der rechten oberen Ecke lassen sich Ausgangs- und Endpunkt der Linie erkennen. Ohne äußere Begrenzung und ohne Fundamentierung schwebt das Gebilde bezugslos im Raum, was durch die Schrägstellung der Skizze auf dem Blatt noch verstärkt wird. Die strudelnden, mäandrierenden Linienzüge in Schlangenform erzeugen ein strukturelles Geflecht, das die dargestellte Architektur nur ahnen lässt.

Haptische Imagination Mit ihrer Verweigerung von anschaulicher, gegenständlicher Repräsentation knüpfen Hadids Skizzen indirekt an jene kritische Theorie der Architekturzeichnung an, die I93 I von dem deutschen Kunsthistoriker Carl Linfert entwickelt und anschließend von Walter Benjamin popularisiert wurde. ${ }^{33}$ In seiner umfänglichen Untersuchung versuchte Linfert, die Architekturzeichnung als eigenständige Kunstgattung zu etablieren, indem er sie auf die Möglichkeiten ihrer taktilen Wahrnehmung hin befragte. Dabei betonte er, dass sich die physische Gestalt der Architektur nur körperlich, sukzessiv im Durchschreiten erschließen lasse: „Denn durch Benutzung erst, durch Eintreten in ein Bauwerk, durch Entlanggehen wird die Architektur für den Menschen wirklich faßbar."34 Aus diesem Grund erschien Linfert die perspektivische Repräsentation von Architektur, die einen festen Betrachterstandpunkt voraussetzt, als unzureichend: „Architektur als solche ist 


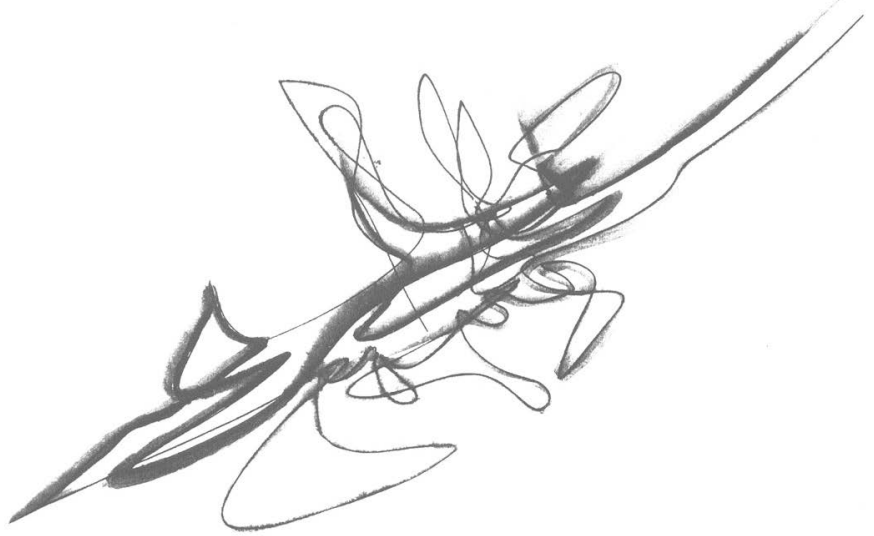

10 Zaha Hadid, Pierres Vives, Montpellier, 2002-2012 (Skizze)

nicht ,Bild‘. Der Raum in seiner Einheit kann nie durch bloß eine Richtung als ein Bild mit einem Mal gesehen und erfaßt werden, denn er ist ringsum. "35 Nicht das Gesehenwerden eines Bauwerkes sei also das Entscheidende bei der Architekturbetrachtung, sondern die ,objektive Einwirkung des Bauwerkes auf das gesamte körperliche und vorstellungsmäßige Sein des Betrachters“". ${ }^{6}$

Um den tektonischen und unbildlichen Charakter der Architektur erfahrbar zu machen, forderte Linfert zeichnerische Verfahren, die sich der malerischen Raumdarstellung und Perspektive widersetzen. Wie vorweggenommene Beschreibungen der Hadid'schen Skizzen muten seine Ausführungen an, wenn er den ambivalenten Eindruck von Einbeziehung bei gleichzeitigem Getrenntsein beschreibt, den der Betrachter dem zeichnerischen Geschehen gegenüber empfindet: „Bei der architektonischen Formung, also auch Zeichnung, entscheidet zwar ein visuelles, aber vom Hinblicken, das auf Beschauer und Blickpunkt festgelegt ist, im Grunde unabhängiges Umreißen der Formvorstellung. Die Konstante der Architekturvorstellung wird nicht in einen Hinblickpunkt verlegt, sondern in ein visuelles Kreisen um das Bauwerk“". ${ }^{37}$

Diese Vorstellung des visuellen Kreisens um Architektur nahm Hadid geradezu wörtlich, wenn sie ununterbrochen Linien in rotierender Bewegung skizzierte. Ihre Zeichnungen vermeiden Konturen, die den Blick des Betrachters auf einen Fluchtpunkt lenken. Sie verweisen auf jenes Kriterium der ,bildunmäßigen“ 
Darstellung, das Linfert für die Architekturzeichnung erhoben hat. Statt Flächen zu umreißen, definieren die kreisenden Linien Strukturen, welche eher auf die taktile als auf die visuelle Rezeption des Betrachters zielen. Indirekte Impulse erhielt diese Form des Zeichnens von Linferts strukturalistischer Wahrnehmungstheorie: „Nur erfordert ein Architekturraum mehr als das Auge, um ganz erfaßt zu werden. Schon das Auge muß weniger sehen als Strukturen durchspüren." ${ }^{8} 8$ Mit seiner emphatischen Formulierung verwies Linfert auf den epistemologischen Anspruch an die Architekturzeichnung. ,Strukturen zu durchspüren' heißt, die Möglichkeiten eines analytischen Sehens auszuspielen, um Einsicht in die strukturellen und funktionalen Zusammenhänge gewinnen zu können, die jenseits der sichtbaren Oberfläche wirken.

Um ein solches strukturales Wahrnehmen zu initiieren, setzte Hadid ein kompositorisches Verfahren ein, das verschiedene Projektionsebenen gleichzeitig aktiviert. Auf einer Skizze des Museo Nazionale delle Arti del XXI Secolo ist über der Aufsicht eine Seitenansicht eingetragen, was allerdings erst offenkundig wird, wenn Modell und Lageplan hinzugezogen werden. ${ }^{39}$ Die gleichzeitige Abbildung von Ansicht und Aufsicht auf einem Blatt Papier zur Darstellung von Architektur ist allgemein akzeptiert. Ungewöhnlich ist allerdings, dass die strukturell ähnlichen Linienfiguren ohne räumliche Umgebung dargestellt sind, so dass der Betrachter kaum zwischen Ansicht und Aufsicht unterscheiden kann. In ihrer Zusammenführung verschiedener Blickpunkte und in ihrer Dekontextualisierung entsprechen Hadids Figuren auf überraschende Weise Linferts Forderung nach einer Aufhebung der bildhaften Fixierung von Architektur. Sie offenbaren dem Betrachter vielmehr jene Differenz zwischen Bild und Gebilde, auf die es Linfert ankam: Während beim Bild eine imaginierte Architektur mit Linien in die Fläche gebracht wird, entsteht beim Gebilde die Architektur als Linienspur der sich bewegenden Hand. Handelt es sich beim Bild lediglich um eine Wiedergabe der architektonischen Erscheinung, zeigt das Gebilde die tektonische Struktur, die im Zeichenprozess erst hervorgebracht wird. In diesem Spannungsfeld von Konstruktion und Aktivität lässt sich neben der architektonischen Zeichnung auch die Kalligrafie verorten, die für Hadid Inspirationsquelle der Architektur war. Im Übergang vom Bild zur haptischen Imagination berühren sich Zeichnen und Schreiben auf das engste. 


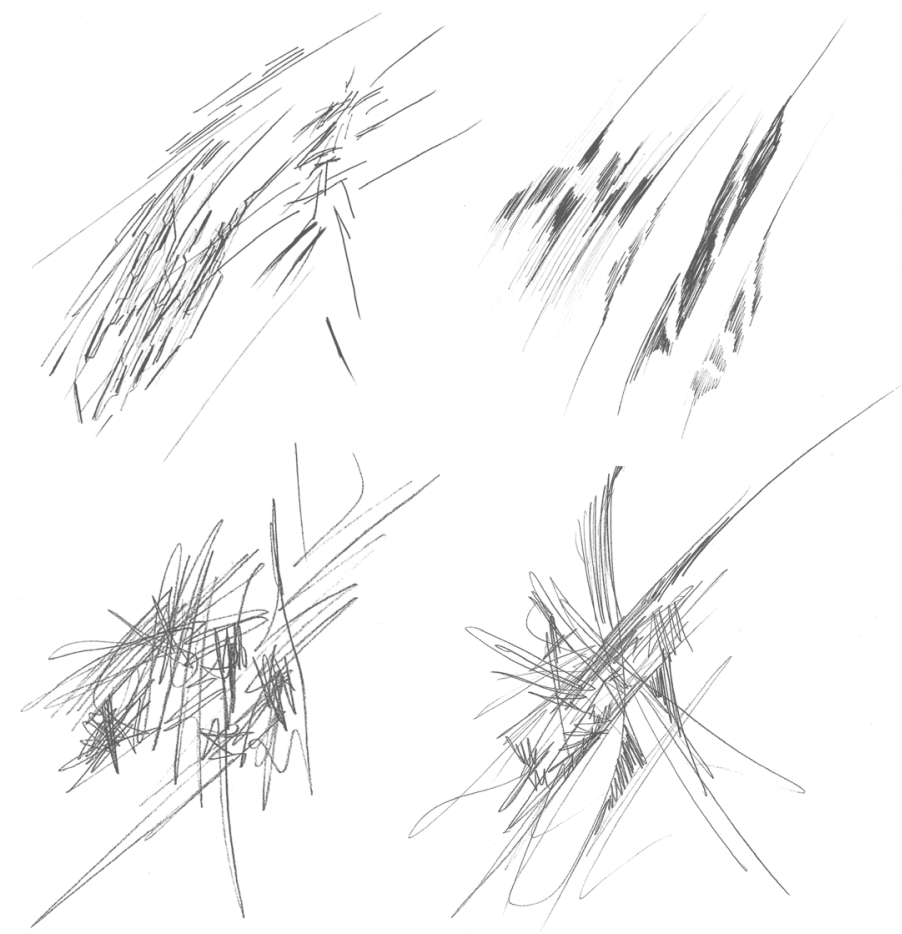

11 Zaha Hadid, La Grande Mosquée, Straßburg, 2000 (Skizzen)

Eigensinnige Linien Hadids Zeichnungen bewegen sich zwischen Abstraktion und Erkennbarkeit. Die Gesamtform ihrer Figuren lässt zwar erahnen, dass es sich bei dem Gegenstand der Darstellung um Architektur handelt, aber der Rhythmus der Linien scheint einem eigenen inneren Antrieb zu folgen, der unabhängigen Gesetzen und Energien gehorcht. Ihre schnellen Kritzeleien und ihre meditativen Kreiszeichnungen wirken so, als ob sich die Zeichnerin von der Linie des Striches lenken ließ - bis sich die Linien vom dargestellten Gegenstand zu lösen und frei zu schwingen begannen (Abb. I I und I 2). Im Gegensatz zu ihren frühen Zwangskonstrukten, die Hadid aus zersplitterten und zugleich höchst kontrollierten Figuren zusammensetzte, verhandelte sie in ihren späteren Skizzen weniger gebundene Formen, die den Eindruck erzeugten, als ob sie sich selbst überlassen seien. 


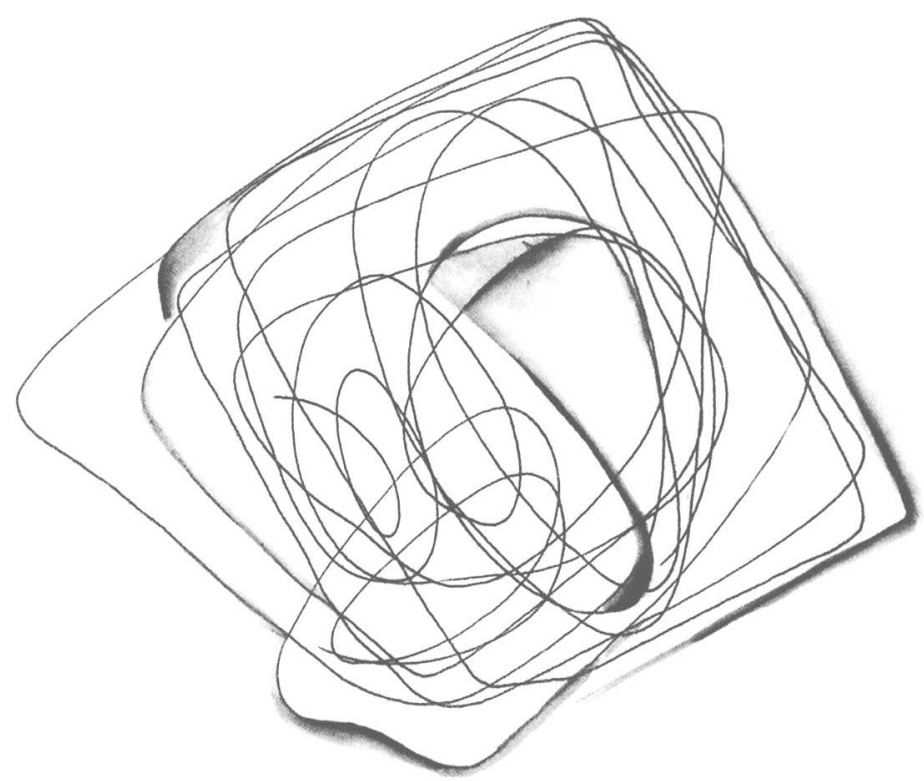

12 Zaha Hadid, Erweiterung des Victoria \& Albert Museum Boilerhouse, London, 1996 (Skizze)

Die Vorstellung von der Eigenbewegung der Linie begann virulent zu werden, als die Mitarbeiterinnen und Mitarbeiter von Hadid für die Umsetzung ihrer Entwürfe zunehmend 3D-Modellierungs- und Animationsprogramme einsetzten, die Formbildungsprozesse in großem Umfang automatisierten..$^{40}$ Zentraler Bestandteil dieser Programme war der interaktive, parametrische, das heißt auf vorprogrammierten Regeln basierende Entwurf, bei dem sich geometrische Elemente in einer zirkulären Abhängigkeit befinden. Unter Zuhilfenahme solcher Techniken entwickelt der Architekt oder die Architektin die Form nicht mehr direkt, sondern gibt Bedingungen und Regeln vor, nach denen die Form selbsttätig entsteht. Mit der Bestimmung der digitalen Form als ein sich selbst dirigierendes System wandelten sich auch die manuellen Zeichnungsformen von Hadid - weg von streng komponierten, multiperspektivisch konstruierten Bildern, und hin zu kontinuierlichen Gebilden, die durch eine strukturelle Eigenbewegung gekennzeichnet sind.

Mit der digitalen Gestaltvision der sich selbst erzeugenden Form rückte zugleich eine Bildtradition in den Vordergrund, in 
welcher der Eigenlauf der Linie als eine Bedingung des Schöpferischen angesehen wurde. Es ist das Prinzip der unentwegten zeichnenden Tätigkeit, des unbestimmten Kritzelns, das auf die Gestik und Materialität des Schreibens und Zeichnens verweist. ${ }^{4}$ In besonderer Weise wurde diese Tradition durch Dürer geprägt. In seinen Randzeichnungen zum Gebetbuch Kaiser Maximilians I. von I5I5 führte er Ornamente, Buchstaben und Figuren in einer arabesken Struktur zusammen. ${ }^{42}$ Die Seitenränder sind mit einer Vielzahl unterschiedlicher Schlangenlinien bedeckt, die sich kontinuierlich verwandeln und so die verschiedenen Bildmotive in ein gleichrangiges Verhältnis setzen. Angesiedelt in den sich überschneidenden Randbezirken von Bild und Schrift sind sie noch nicht oder nicht mehr lesbar. Hadids dynamische Linienzüge weisen eine strukturelle Verwandtschaft mit den ausschweifenden Kurvierungen Dürers auf. Mit ihren nicht-hierarchischen Linienbildungen unterlief die Zeichnerin in ähnlicher Weise traditionelle Motivordnungen und führte bisher getrennte Wahrnehmungskategorien wie Figur und Grund, Zentrum und Rand zusammen.

Die Idee der eigenläufigen Totallinie, die alles miteinander verbindet, fand schließlich ihren Höhepunkt darin, dass Hadid die

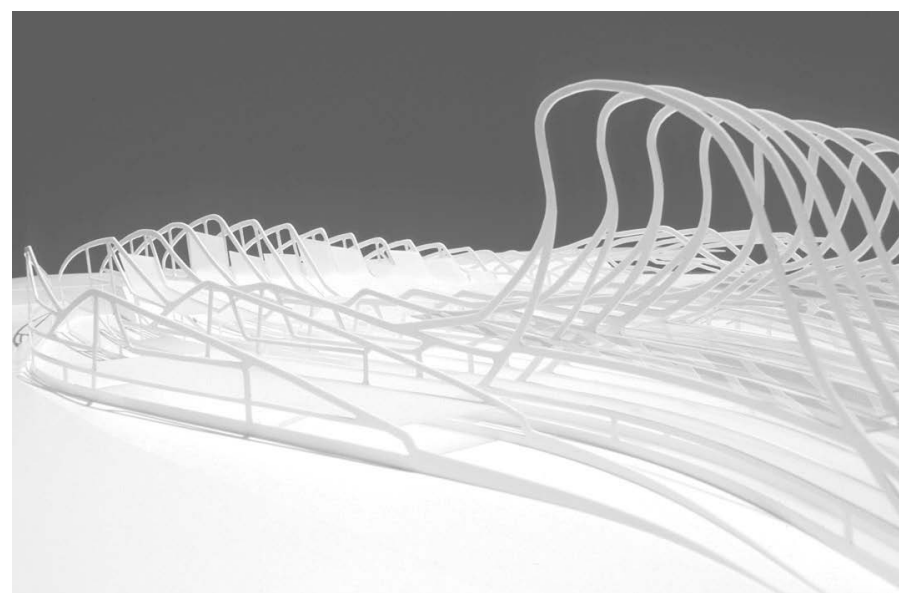

13 Zaha Hadid, La Grande Mosquée, Straßburg, 2000 (Strukturmodell) 
gezeichneten Linien von der Fläche löste und räumlich werden ließ (Abb. I3). Dabei verwendete sie die kalligrafisch inspirierten Liniengebilde nicht als Schmuck-, sondern als Strukturformen. Für eine Moschee in Straßburg entwarf sie eine bauliche Struktur, die aussah, als ob sie ein kalligrafisches Ornament in den Raum projiziert hätte (Abb. I4). Sie überzog den städtischen Grund mit aufgewölbten, sich überlappenden Bändern und setzte so schriftähnliche Ornamente in bisher nicht gekannter Größenordnung und Totalität dreidimensional um. ${ }^{43}$

Die zentrale Vision, welche die kalligrafisch anmutende Zeichentätigkeit Hadids beflügelte, war es, Ornamente als aktive Bauformen einer neuen kodifizierten und physischen Realität zu entwickeln, in der sich Natur und Kultur unauflöslich verbinden. Auch hierfür können die digitalen Entwurfs- und Fertigungstechniken als Impulsgeber gelten, denn sie befördern die Ornamentbildung durch automatisierte Verfahren der Elementvariation und Flächentesselation und ermöglichen die Verschränkung bisher getrennter Produktionsformen in der individuellen Serienfertigung. Zugleich lassen sich die digital motivierten Linienornamente in einen größeren historischen Kontext stellen: Der Idee der dynamischen ornamentalen Struktur liegt eine Vorstellung der geometrisch-abstrakten Moderne zugrunde, die sich gegen das Ornament als etwas Appliziertes, Schmückendes und Nicht-Funktionales richtet. Es ist ein Ornamentbegriff, der gegen Ende des I9. Jahrhunderts von dem amerikanischen Architekten Louis Sullivan geprägt und im architektonischen Strukturalismus der I950er und 6oer Jahre weiterentwickelt wurde. ${ }^{44}$ Ornament wird in dieser Perspektive nicht als Dekor oder addiertes Muster verstanden, sondern als elementare Ordnungsform, die vom Trägermedium losgelöst ist, oder genauer, mit dem Trägermedium verschmilzt. Hierbei wird die ordnende Funktion des Ornamentalen als ein ordinare gegenüber der schmückenden Funktion des Ornaments als ein ornare unterschieden. In diesem Begriff des Ornaments als aktiver Ordnungssinn liegt der zentrale Anknüpfungspunkt für die linear-strukturelle Architektur Hadids.

Hadids ornamentale Strukturen sind Produkte kontinuierlicher Handbewegungen, die unentwegt Formen generieren. Ihr Bestreben, hinter den Strukturen die Bewegung zu gestalten, lässt darüber hinaus an die Ornamentdefinition von Niklas Luhmann denken. Der Systemtheoretiker sah im Ornament „die Grundform 


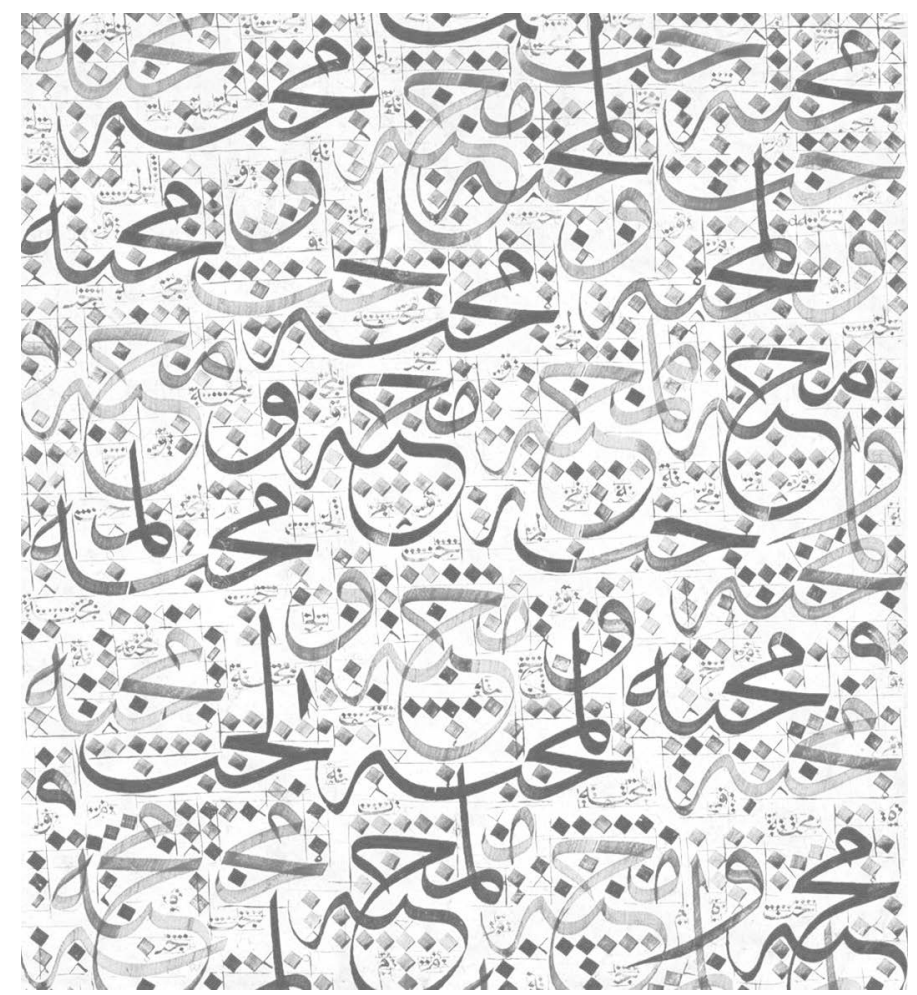

14 Mehmed Özçay, Mahabbah (Liebe), Schriften: Jali Thulth, Jali Naskh, 1420/2000 (Tinte auf Papier) Detail

des Entwickelns von Formen aus Formen“, womit er eine Reformulierung des Ornamentbegriffs vorschlug. ${ }^{45}$ Er relativierte den gestaltorientierten Sinn und empfahl einen prozessorientierten Ornamentbegriff, unter dem sich die zeichnerischen Metamorphosen von Hadid unmittelbar fassen lassen.

Diesen grundlegenden Perspektivwechsel vom schmückenden Ornament zum ornamentalen Prozess veranschaulicht auf eine nochmals gesteigerte Weise Hadids Sprungschanze in Innsbruck (Abb. I5). Die Zeichenhaftigkeit dieses Sportbaus weckt Assoziationen an kalligrafische Buchstaben aus dem arabischen Alphabet. So lässt sich in dem gebauten Raumknoten etwa die Verschränkung von Alif und Ain - des ersten und achtzehnten Buchstabens des arabischen Alphabets - erkennen (Abb. I6). Der Sportbau ist 


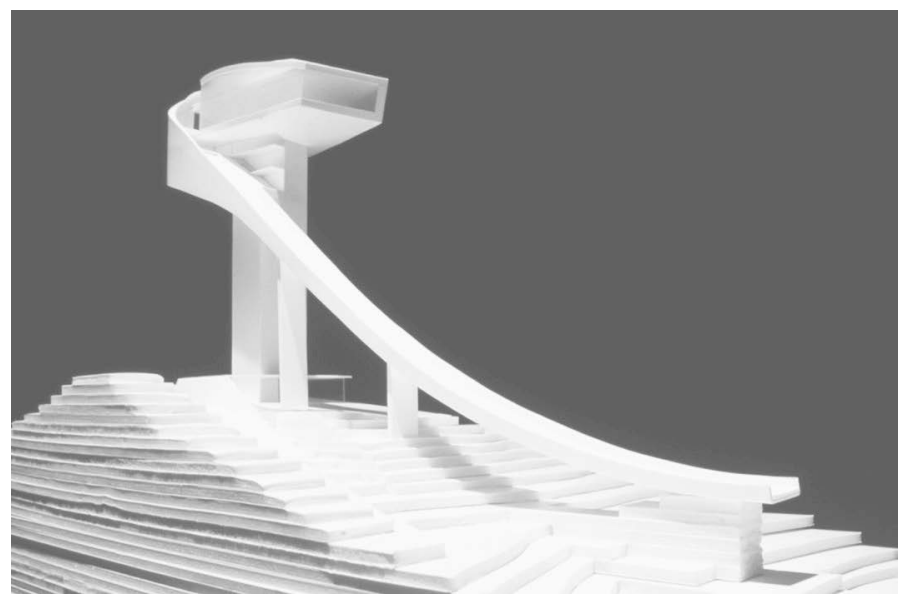

15 Zaha Hadid, Bergisel Skisprungschanze, Innsbruck, 1999-2002 (Modell)

ein Beispiel dafür, wie die Bewegung der zeichnenden Hand in einem monumentalen Raumzeichen gerinnt, das wiederum zu einer körperlich performativen Reaktion, einem Skisprung, anregt. Die Gestalt von Turm und Schanze folgt der schwingenden Handbewegung, sie drückt Bewegung als Formcharakter aus und bedingt zugleich die Flugbewegung des Nutzers (Abb. I7). Die Totallinie mündet so in eine Totalbewegung.

Körperhafte Bildproduktion Hadid ging es bei ihren zeichnerischen Experimenten um die Hervorbringung bisher ungesehener, neuer Formen in der Architektur. Um zu innovativem Formmaterial zu gelangen, griff sie bewusst nicht-architektonische Traditionen der Zeichnung und der Schrift auf. Dabei bezog sie sich weniger auf den geschriebenen Buchstaben, das Wort, den Text, der in einen Bildkontext integriert wird. Vielmehr ging es ihr um die Lust an der Geste des Schreibens, die Linien und Linienführung wie geschrieben vorführt, ohne dass lesbarer Sinn darin entdeckt werden kann. Die Geste des zeichnenden Schreibens überführte Hadid dann kontinuierlich in eine architektonische Geste, die wiederum von Bewegung spricht und Bewegung hervorruft.

Ihre in sich gedrehten Raumkörper fordern dazu heraus, das Ver-hältnis von Bild und Bau zu überdenken. ${ }^{46}$ Bisherige Beschrei- 


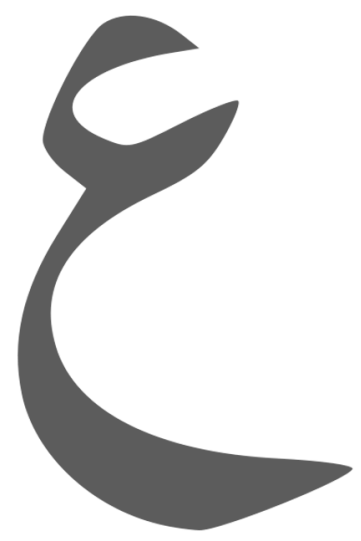

16 Alif und Ain, der erste und achtzehnte Buchstabe im arabischen Alphabet

bungen bildlicher Qualitäten von Architektur zielten vor allem auf zeichenhafte Gebäude oder Bauteile, die sich durch mehr oder weniger bestimmte programmatische Konnotationen auszeichnen, sowie auf flächenhafte Ansichten von Bauten, insbesondere auf Fassaden. Die bildlichen Qualitäten der Hadid'schen Architektur beziehen sich hingegen nicht auf Bildzeichen und semiotische Ansätze seit den I970er Jahren, sondern auf körperhafte Bilderfahrungen. ${ }^{47}$ In diesem Sinne von einer Bildlichkeit der Architektur zu sprechen, meint Seherlebnisse und raumzeitliche Dimensionen, die den Betrachter für kurze Zeit aus der gewohnten Ordnung des alltäglichen Sehens und Bewegens heraustreten lassen.

Im Entwurf komplexer Interaktionen von Bild, Bau und menschlichem Körper sowie in der Visualisierung raumzeitlicher Strukturen und Prozesse nimmt der Computer einen besonderen Rang ein. In der Tat haben digitale Entwurfsverfahren zahlreiche Funktionen der Zeichnung aufgenommen und ersetzt. Hadids bewegte Architekturobjekte hätten kaum erdacht und errichtet werden können, wenn sie nicht durch avancierte Modellierungsprogramme simuliert, errechnet und gefertigt worden wären. Die Möglichkeit, Räume zu imaginieren, die über die Grenzen des Vorstellungsvermögens hinausgehen, basierte für die Architektin aber weiterhin und unabänderlich auf der Handzeichnung. Der 


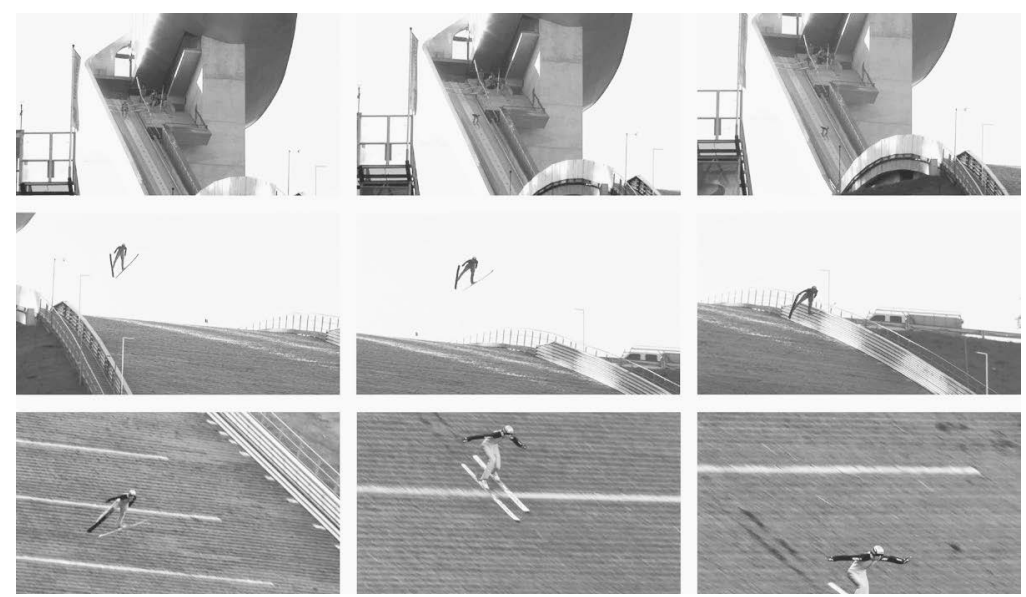

17 Zaha Hadid, Bergisel Skisprungschanze, Innsbruck, 1999-2002 (Stills)

Vorgang des Skizzierens darf nicht unterschätzt werden, denn in ihm gewann Hadid Formen und Strukturen, die den digitalen Produktionsprozess und die bauliche Umsetzung der entworfenen Architektur überdauerten. In diesem Raum primärer Formation behauptet sich das Zeichnen auch in hochtechnischen Umgebungen. Es ist der Raum, in dem noch unbekannte Vorstellungen fixiert, gedankliche Verfestigungen gelöst und Alternativen formuliert werden. Hadids Hinwendung zur Zeichnung ist daher nicht als Maßnahme gegen die zunehmende Digitalisierung des Entwerfens zu verstehen, sondern als notwendige Ergänzung. Damit verfolgte sie eine Praxis des Entwerfens, die bis heute relevant ist, weil sie dazu ansetzt, die machtvolle habituelle Grenzziehung zwischen haptisch-manuellen und visuell-digitalen Entwurfsverfahren zu überwinden.

Angesichts des Zusammenspiels dieser Verfahren drängt sich die Frage auf, wie digitale Technologien dem Verlust der Fähigkeit begegnen können, mit der Hand zu schreiben und zu zeichnen. Kann es zukünftig computerbasierte Werkzeuge des Entwerfens geben, die - wie es der britische Anthropologe Timothy Ingold erhofft - ,nicht notwendigerweise auf Kosten des Empfindens gehen müssen" $?^{48}$ Anstatt Tastaturen und Computermäuse zur Ersetzung der schreibenden und zeichnenden Hand zu bauen, sollten nach Ingold zukünftig ,digital erweiterte Stifte [entwickelt 
werden], welche unsere Sinnlichkeit auf die Nuancen der Linien oder die Qualitäten der Papieroberfläche konzentrieren, dann könnten wir vielleicht das Beste aus beiden Welten erreichen“. ${ }^{49}$

Anmerkungen

1 Vgl. Jörg H. Gleiter, "Gegenstandsversprechen. Entwerfen als Prozess der Theoriebildung", Vortrag im Rahmen der Tagung „Manifestationen im Entwurf in Design, Architektur und Ingenieurwesen - eine interdisziplinäre Bestandsaufnahme", RWTH Aachen, 10.-12. April 2014.

2 Jörg. H. Gleiter, „Vom Abreißen der Modellierungskette. Entwerfen im digitalen Zeitalter", in: Sabine Ammon, Inge Hinterwaldner (Hg.), Bildlichkeit im Zeitalter der Modellierung. Operative Artefakte in Entwurfsprozessen der Architektur und des Ingenieurwesens, München 2017, S. 89-102.

3 Siehe Mario Carpo, The Alphabet and the Algorithm, Cambridge, Mass. 2011, S. 44-45.

4 Vgl. Karin Krauthausen, Omar W. Nasim (Hg.), Notieren, Skizzieren. Schreiben und Zeichnen als Verfahren des Entwurfs, Zürich, Berlin 2010.

5 Grundlegend: Bernd Mahr, „Ein Modell des Modellseins. Ein Beitrag zur Aufklärung des Modellbegriffs", in: Ulrich Dirks, Eberhard Knobloch (Hg.), Modelle, Berlin 2008, S. 187-218; Horst Bredekamp, "Frank Gehry and the Art of Drawing", in: Mark Rappolt, Robert Violette (Hg.), Gehry draws, London 2004, S. 11-28; Horst Bredekamp, "Modelle der Kunst und der Evolution", Debatte 2, 2005, S. 13-20; Reinhard Wendler, Das Modell zwischen Kunst und Wissenschaft, Paderborn 2013.

6 Horst Bredekamp, zit. n. Thomas Kleinspehn, Wie Bilder auf Menschen wirken. Horst Bredekamp: "Theorie des Bildakts", 21. September 2011, unter: http://www.deutschlandfunk.de/ wie-bilder-auf-menschen-wirken.700.de.html?dram:article id $=85245$ [01. Juni 2019].

7 Siehe Bredekamp, Frank Gehry (Anm. 5), S. 15-17.

8 Bredekamp, Modelle (Anm. 5), S. 14.

9 Siehe Robert E. Somol, „Dummy Text, or The Diagrammatic Basis of Contemporary Architecture", in: Peter Eisenman, Diagram Diaries, New York 1999, S. 6-25.

10 Sybille Krämer, Jan Wöpking, „Plenartagungsbericht der Forschergruppe D-III-1 ,Diagrams'", in: Friederike Fless, Gerd Graßhoff, Michael Meyer (Hg.), Berichte der Forschergruppen auf der Topoi- 
Plenartagung 2010, eTopoi. Journal for Ancient Studies, Sonderband 1, 2011, S. 1, unter: http://www.topoi.org/wp-content/ uploads/2013/05/13-70-2-PB.pdf [01. Juni 2019].

11 Siehe Nicole E. Stöcklmayr, "Architektur ohne Maßstab. Digitale Visualisierungen im Entwurfsprozess", in: Ingeborg Reichle, Steffen Siegel (Hg.), Maßlose Bilder. Visuelle Ästhetik der Transgression, München 2009, S. 279-294, hier S. 285.

12 Patrik Schumacher, zit. n. Zaha Hadid, Patrik Schumacher, „Es gibt keine Gesellschaft ohne Architektur. Zaha Hadid und Patrik Schumacher äußern sich über Arbeit, Karriere und Ästhetik", Neue Zürcher Zeitung, 13. August 2011, unter: http://www.nzz.ch/ es-gibt-keine-gesellschaft-ohne-architektur-1.11889746 [01. Juni 2019].

13 Zaha Hadid, Jessica Hines, "In her own words: Zaha Hadid", DailyO, 01. April 2016, unter: http://www.dailyo.in/arts/zaha-hadidartchitecture-public-spaces-pritzker-architectural-prize-design-calligraphy/story/1/9832.html [01. Juni 2019].

14 Zaha Hadid studierte zunächst Mathematik an der American University of Beirut im Libanon und emigrierte dann nach Großbritannien, wo sie ein Architekturstudium an der renommierten Architectural Association School of Architecture in London aufnahm.

15 George Spencer-Brown, Laws of Form, New York 1979, S. 3.

16 Siehe Hans Dieter Huber, „,Draw a distinction.' Ansätze zu einer Medientheorie der Handzeichnung", in: Deutscher Künstlerbund e.V. (Hg.), Zeichnen. Der deutsche Künstlerbund in Nürnberg 1996. 44. Jahresausstellung, Ausst.kat. (Nürnberg, Germanisches Nationalmuseum, 1996/1997), Berlin 1996, S. 8-21, unter: http://www.hgbleipzig.de/ARTNINE/huber/aufsaetze/handzeichnung.html [01. Juni 2019].

17 Siehe Vicente Carducho, Dialogos de la pintura. su defensa, origen, esse[n]cia, definicion, modos y diferencias, Madrid 1633, unter: https://archive.org/details/gri_dialogosdela00card, S. 485 [01. Juni 2019]. Zur Deutung von Carduchos Zeichnung siehe: Bredekamp, Frank Gehry (Anm. 5), S. 11.

18 Siehe Gordana Fontana-Giusti, Patrik Schumacher (Hg.), Zaha Hadid. The Complete Works, Bd. 4: Process. Sketches and Drawings, New York 2004, S. 66-67.

19 Zaha Hadid, zit. n. Peter Noever, "Architektur für eine schöne neue Welt. Zu Zaha Hadids Wiener Ausstellung / Changing Architecture. On Zaha Hadid's Vienna Exhibition", in: Ders. (Hg.), Zaha Hadid 
Architektur / Architecture, Ausst.kat. (Vienna, Austrian Museum of Applied Arts / Contemporary Art, 2003), Ostfildern-Ruit 2003, S. 10.

20 Vgl. Galerie Gmurzynska (Hg.), Zaha Hadid and Suprematismus, Ausst.kat. (Zürich, Galerie Gmurzynska, 2010), Berlin 2012, S. 35-36.

21 Zaha Hadid, Philip Stevens, „Interviews with Zaha Hadid. The Architect's Work in Her Own Words", Designboom, 31. Mai 2016, unter: http://www.designboom.com/architecture/zaha-hadid-interview-quotes-dies-aged-65-03-31-2016 [01. Juni 2019].

22 Siehe Gabriele Gramelsberger, "Story Telling with Code", in: Andrea Gleiniger, Georg Vrachliotis (Hg.), Code. Zwischen Operation und Narration, Basel 2010, S. 29-40, hier S. 37.

23 Grundlegend: Horst Bredekamp, „Die Unüberschreitbarkeit der Schlangenlinie", in: Christian Schneegass, Akademie der Künste (Hg.), minimal - concept. Zeichenhafte Sprachen im Raum, Amsterdam, Dresden, Berlin 2001, S. 205-208; Werner Hofmann, Die Schönheit ist eine Linie. 13 Variationen über ein Thema, München 2014.

24 Siehe Leon Battista Alberti, „De pictura”, in: Ders., Das Standbild. Die Malkunst. Grundlagen der Malerei. De Statua. De Pictura. Elementa Picturae, hg. u. übers. v. Oskar Bätschmann, Christoph Schäublin, Darmstadt 2000, S. 278 (Par. 45).

25 Albrecht Dürer, Underweysung der messung, mit dem zirckel un[d] richtscheyt, in Linien ebnen unnd gantzen corporen, Nürnberg 1525, S. A II, unter: https://digital.slub-dresden.de/data/ kitodo/vndedemem_27778509X/vndedemem_27778509X_tif/ jpegs/00000007.tif.large.jpg [01. Juni 2019].

26 Albrecht Dürer, Alfred Peltzer, Albrecht Dürer's Unterweisung der Messung. Um Einiges gekürzt und neuerem Sprachgebrauch angepaßt, München 1908, S. 18.

27 Siehe William Hogarth, The Analysis of Beauty. Written with a View of Fixing the Fluctuating Ideas of Taste, London 1753, Titelblatt, unter: https://archive.org/details/analysisofbeauty00hoga [01. Juni 2019].

28 Paul Klee, Pädagogisches Skizzenbuch, Bauhausbücher, Bd. 2, München 1925, S. 6, Fig. 1.

29 Gilles Deleuze, Félix Guattari, Rhizome. Introduction, Paris 1976, S. 41. Deutsche Übersetzung: Dies., Rhizom, übers. v. Dagmar Berger, Clemens-Carl Haerle, Helma Konyen u. a., Berlin 1992, S. 41.

30 Vgl. Gmurzynska (Hg.), Zaha Hadid (Anm. 20).

31 Siehe Christoph Otterbeck, Europa verlassen. Künstlerreisen am Beginn des 20. Jahrhunderts, Köln, Weimar, Wien 2007, S. 103-109; 
Roger Benjamin, Kandinsky and Klee in Tunesia, Oakland, CA 2015, S. 179.

32 Wassily Kandinsky, "Über die Formfrage” (1912), in: Ders., Franz Marc (Hg.), Der Blaue Reiter, 2. Aufl., München 1914, S. 74-100, hier S. $85-86$.

33 Vgl. Carl Linfert, „Die Grundlagen der Architekturzeichnung. Mit einem Versuch über französische Architekturzeichnungen des 18. Jahrhunderts", Kunstwissenschaftliche Forschungen, Bd.1, Berlin 1931, S. 133-246; Walter Benjamin, „Strenge Kunstwissenschaft. Zum ersten Bande der ,Kunstwissenschaftlichen Forschungen' (Erste und zweite Fassung) (1931)", in: Ders., Gesammelte Schriften, Bd. 3, Frankfurt a. M. 1972, S. 367-369, 373-374, 652-658.

34 Linfert, Architekturzeichnung (Anm. 33), S. 141.

35 Ebd., S. 144.

36 Ebd.

37 Ebd., S. 135.

38 Ebd., S. 143.

39 Siehe Zaha Hadid Architects, unter: www.zaha-hadid.com/ wp-content/uploads/2019/12/965_maxxi_sket_0354-437x608.jpg [01. Juni 2019]. Modell und Lageplan sind abgebildet in: Zaha Hadid, Aaron Betsky, The Complete Zaha Hadid. Expanded and Updated, London 2013, S. 106.

40 Vgl. Kevin Holden Platt, Patrik Schumacher, „Interview: Patrik Schumacher", Arcspace, 01. Mai 2014, unter: http://www.arcspace. com/articles/interview-patrik-schumacher [01. Juni 2019].

41 Vgl. Christian Driesen, Rea Köppel, Benjamin Meyer-Krahmer u. a. (Hg.), Über Kritzeln. Graphismen zwischen Schrift, Bild, Text und Zeichen, Zürich 2012.

42 Siehe Kaiser Maximilian I., Albrecht Dürer [Bearb.], Oratio ad suu[m] proprium angelu[m], [Augsburg], 1515, fol. 42 verso, unter: http:// daten.digitale-sammlungen.de/ db/0008/bsb00087482/images/ index.html?fip=193.174.98.30\&seite=94\&pdfseitex= [01. Juni 2019].

43 Dass Hadid mit ihren ikonischen Gebäudeskulpturen auch nationalistische Bedürfnisse nach repräsentativen Bauten erfüllte, zeigten ihre zahlreichen Bauaufträge in autoritär regierten Ländern, die ihr in den letzten Lebensjahren massive öffentliche Kritik einbrachten. Beispielhaft: Marion Löhndorf, „Die Architektin Zaha Hadid in der Kritik. Bau-Ikonen für autoritäre Staaten", Neue Zürcher Zeitung, 22. Juli 2014, unter: http://www.nzz.ch/feuilleton/kunst_architektur/ bau-ikonen-fuer-autoritaere-staaten-1.18347899\#kommentare [01. Juni 2019]. 
44 Vgl. Louis H. Sullivan, "Ornament in Architecture (1892)", in: Ders., Kindergarten Chats (Revised 1918) and Other Writings, New York 1968, S. 187-190. Siehe auch Carolin Höfler, „Das digitale Ornament als universale Form. Mediale Strategien der Vereinheitlichung", in: Annette Tietenberg (Hg.), Muster im Transfer. Ein Modell transkultureller Verflechtung?, Köln, Wien 2015, S. 71-88.

45 Niklas Luhmann, Die Kunst der Gesellschaft, Frankfurt a. M. 1995, S. 193; siehe auch Michael Dürfeld, Das Ornamentale und die architektonische Form. Systemtheoretische Irritationen, Bielefeld 2008.

$46 \mathrm{Vgl}$. Andreas Beyer, Matteo Burioni, Johannes Grave (Hg.), Das Auge der Architektur. Zur Frage der Bildlichkeit in der Baukunst, München 2011.

47 Vgl. Markus Rath, Jörg Trempler, Iris Wenderholm (Hg.), Das haptische Bild. Körperhafte Bilderfahrung in der Neuzeit, Berlin 2013.

48 Tim Ingold, Petra Löffler, Florian Sprenger, „Eine Ökologie der Materialien. Ein E-Mail-Interview über Korrespondenz, Resonanz und Besessenheit sowie über den Nutzen, Gelehrsamkeit und Handwerk zu verbinden", Zeitschrift für Medienwissenschaft 14, 1/2016, S. 87-94, hier S. 93.

49 Ebd. 


\section{Skizzen von Diagrammen und Diagramme von Skizzen}

Wenn man konventionelle Architekturzeichnungen im Kontext neuer digitaler Medien hinterfragt, entdeckt man, dass das Zeichnen zum einen ,mutiert“ ist und beinahe mühelos mit dem Computer einhergeht, zum anderen jedoch weiterhin eine analoge Tätigkeit mit Stift und Papier darstellt. Trotz dieser „Mutation“ trotz der Veränderungen von Abhängigkeiten zwischen digitalen und analogen Geräten und Notationsformen - scheint im Zusammenhang mit dem architektonischen Zeichnen eine Art diagrammatisches Denken bestehen zu bleiben, das sich nicht wesentlich verändert hat. Dieses Denken ist zum einen mit der Tätigkeit des Skizzierens verbunden und erstreckt sich andererseits vom Entwerfen bis in generative, künstlerische Schaffensprozesse.

Dieser Beitrag möchte aufbauend auf dem Diagramm-Begriff der Philosophen Charles S. Peirce, Gilles Deleuze und Nelson Goodman das Denken, das sich im architektonischen Zeichnen vollzieht, sowohl diskursiv als auch durch Zeichnungen erläutern. Dabei werden analoge wie auch digitale Verfahren betrachtet sowie mit eigenen Zeichnungen veranschaulicht. ${ }^{\mathrm{I}}$

Diagrammatisches Denken: Das Diagramm bei Peirce Peirces Diagramm ist vorwiegend ein rationales Mittel zum logischen Denken. Dabei steht seine Idee des diagrammatischen Denkens, vor allem wie es von dem Wissenschaftstheoretiker Frederik Stjernfelt weitergeführt wurde, in direktem Zusammenhang mit Intuition, Sinneseindrücken und der menschlichen Welterfahrung. ${ }^{2}$ Das beruht darauf, dass es abduktives Denken mit einschließt, was nach Peirce sowohl eine unsichere logische Form ist, ${ }^{3}$ ein „Raten“ (guess), als auch die einzige Art des 
Denkens, die irgendetwas Neues im Denkprozess zulässt. ${ }^{4}$ Stjernfelt betont, dass abduktives und deduktives Denken im diagrammatischen Denken zusammenkommen und dass sich dadurch die unsicherste Art der Logik - beinahe nur eine skizzenhafte Form des Denkens - und die stärkste Form logischen Denkens - wie es beispielsweise für die Mathematik typisch ist - überkreuzen. Bei Peirce sind alle Denkformen mit Zeichen verbunden. ${ }^{5}$ Die Verbindung zwischen Gedanken und Zeichen ist auch beim architektonischen Zeichnen von großer Bedeutung, unter anderem weil Architekturzeichnungen starken Konventionen folgen. Grundrisse, Schnitte, Ansichten, Axonometrien und Perspektiven sind konventionelle Zeichentechniken, die klar durch bestimmte Zeichen und graphische Signaturen (sowie unterschiedliche Linienstärken und -typen) kommunizieren. Dennoch ist es gleichzeitig möglich, mit diesen Techniken zu skizzieren, wobei eine Skizze nicht unbedingt klare Zeichen enthält, wie sie zu lesen ist. Wenn dies mit Peirces Taxonomie der Zeichen verglichen wird, ist es so, dass Zeichen, die durch starke Konventionen bewahrt werden, Symbole sind, die eine klare Bedeutung vermitteln und auf deduktivem Denken basieren. Ikone hingegen sind Zeichen, die offen für viele verschiedene Lesarten sind, entsprechend der vagen Idee von Ähnlichkeit, ${ }^{6}$ welche für das Verhältnis zwischen Ikon und dessen Objekt steht. Bei Peirce ist das Diagramm eine Unterkategorie des Ikons. Das Diagramm funktioniert dabei, Stjernfelt zufolge, als solche Unterkategorie nicht als einfache visuelle Ähnlichkeit, sondern vielmehr durch operative Ähnlichkeit. ${ }^{7}$ Dementsprechend ist das Diagramm sehr hilfreich, um über etwas zu reflektieren - zum Beispiel über ein Problem oder ein Gebäude - wie es zusammengesetzt ist und wie es funktioniert. Ein Diagramm macht es möglich, in Gedankenprozessen Relationen von Problemen zu sehen, zu ordnen und umzuordnen, ${ }^{8}$ so wie auch Architekturzeichnungen es ermöglichen, ein Gebäude zu sehen, zu ordnen oder umzuordnen. Eine Zeichnung beginnt oft als eine unbestimmte Skizze und wird dann mehr und mehr in Richtung der Klarheit weiterentwickelt. Diese Fähigkeit architektonischer Zeichnungen kann Stjernfelts Definition des Diagramms zufolge sehr treffend als eine ,skelettartige Skizze von Relationen“ bezeichnet werden. ${ }^{9}$

Ein Diagramm ermöglicht es in wechselseitigem Austausch mit einem Diagrammartefakt zu denken. ${ }^{\text {IO }}$ Eine Architekturzeichnung kann ein solches Artefakt sein: Eine Manifestation, die 
Spuren von Denkprozessen in sich trägt oder sogar die Ergebnisse der Denkprozesse visualisiert. Somit gibt es sowohl Diagrammartefakte als auch ein ,unsichtbares“, vielleicht sogar mentales Diagramm für das Denken: eine Denkfigur. ${ }^{\text {II }}$ Der diagrammatische Denkprozess wird durch diese doppelte Natur aus Abduktion-Deduktion/Artefakt-Denken belebt und wird zu einer generativen Denkform. Dabei führt ein Gedanke zum nächsten Schritt in einem Zusammenspiel aus Manifestation und Denkfigur. Daraus folgt ebenfalls, dass ein Diagramm erkenntniserzeugend ist, ${ }^{\mathrm{I} 2}$ insofern dass etwas, das noch nicht wirklich deutlich ist, deutlicher werden kann. ${ }^{\mathrm{I} 3}$ Diese doppelte Natur von Peirces und Stjernfelts Diagramm, welche sich vom Hervorrufen von Ideen durch eine Art „Skizzendenken“ bis zum logischen Strukturieren von Ideen erstreckt, ist ebenfalls für die Architekturzeichnung charakteristisch. Die Zeichnung ist auf der einen Seite ein völlig eindeutiges Kommunikationssystem, das das Bauen ermöglicht, während sie auf der anderen Seite beim Skizzieren ermöglicht, über Räume nachzudenken, die noch nicht deutlich sind oder noch nicht existieren. Denken in Skizzen trifft sozusagen in Peirces und Stjernfelts Diagrammen auf logisches Denken und kann in dem diagrammatischen Artefakt vermittelt werden: in der Zeichnung.

Denken durch Zeichnungen Die erwähnten Zeichentechniken, wie Grundrisse oder Schnitte können als recht offene Diagramme aufgefasst werden. Zum einen, weil sie verwendet werden können, um sehr verschiedene Gebäude und räumliche Ideen zu erfassen und damit umzugehen, zum anderen, weil sie im Prozess des Skizzierens eines Projektes genauso wie in Phasen klarer Anweisungen nötig sind. In diesem Beitrag befinden sich drei Serien von Zeichnungen, die ich jeweils als Beispiele für Skizzendiagramme betrachte. Diese Serien sind jeweils in Abwechslung zu philosophischen Diagrammkonzepten an ausgewählten Stellen platziert. Sie sollen eine andere Ebene einführen, die sich der Differenzierung des Diagrammkonzepts durch Zeichnung widmet. Zu jeder Zeichnungsserie gibt es deswegen auch einen kurzen erläuternden Text. Die Zeichnungen sind unbestimmt und offen für viele Lesarten, obwohl sie mit formellen und logischen Strukturen arbeiten.

Die konventionellen Zeichentechniken werden von einigen Architekten auch als limitierend betrachtet, zum Beispiel von Bernard Tschumi. Tschumis The Manhattan Transcripts ${ }^{\mathrm{I} 4}$ kann als 
ein Beispiel herangezogen werden, bei dem sich das diagrammatische Denken im Sinne Peirces und Stjernfelts als Architekturzeichnung äußert, wobei Tschumi anstrebte, über die konventionellen Zeichentechniken hinauszugehen und sie zu erweitern.

Hierbei arbeitete er mit anderen Zeichentechniken, bei denen er vor allem von der Montagetechnik und den Storyboards des Filmemachers Sergei Eisenstein inspiriert wurde. ${ }^{15}$ The Manhattan Transcripts ist eine architektonische Untersuchung, ausgeführt in Zeichnungen, die sich mit erdachter Gewalt und leidenschaftlichen Ereignissen in New York beschäftigen. Die Zeichnungsserie ist dreigeteilt in die Schemata Raum, Ereignis und Bewegung (space, event, movement) und in vier Serien von Zeichnungen strukturiert, die mal ein fragmentiertes Narrativ bilden und sich mal einer narrativen Logik komplett entziehen. Tschumis Anregung ist es, die Grenzen der Konventionen der Architekturzeichnungen zu verschieben und es dabei den Aspekten Bewegung und Ereignis zu erlauben, neben Raum, der üblicherweise im Fokus steht, als Teil der Architektur vermittelt zu werden. ${ }^{16}$ The Manhattan Transcripts ist sozusagen Tschumis Art, das konventionelle Diagramm der Architekturzeichnung selbst zu umreißen, um die Konventionen zu hinterfragen und zu erweitern. Tschumi nennt die Manhattan Transcripts sogar ein „device“ und „tool-in-themaking“: ein „Werkzeug“, enthalten in Zeichnungen. ${ }^{17}$ Tschumi kann das „Werkzeug“ nicht zeigen, ohne es zu zeichnen; er muss sein Diagramm zeichnen, bevor es entsteht.

Tschumi erklärt, dass die Zeichnungen mit sogenannten „devices“ hergestellt werden. ${ }^{18}$ Der Begriff , device ' drückt eine objektive Regel oder Anleitung in diskursiver Sprache aus. Es gibt zum Beispiel ,devices` wie Überlagerung oder Stapeln, die dann doch durch ein subjektives Vorgehen beim Zeichnen umgesetzt werden. ${ }^{19}$ Ein , device ist eine Zeichenhandlung in Relation zu einem diskursiven Konzept. Die Qualität eines ,device ' ist es, dass es offen ist und interpretiert werden muss, um als Zeichnung erfasst zu werden. Stapeln ist zum Beispiel keine klare Anweisung, kann aber dennoch eine Zeichenhandlung ermöglichen. Auf der anderen Seite kann Stapeln auf viele Weisen gezeichnet werden und so eventuell dabei helfen, einen Zeichenprozess zu generieren. ,Devices" sind also offene Regeln, die einer subjektiven Interpretation bedürfen und die Entwicklung von Zeichenkonzepten leiten. ${ }^{20}$ Tschumi deckt dabei etwas über die Weise auf, wie er ,devices 

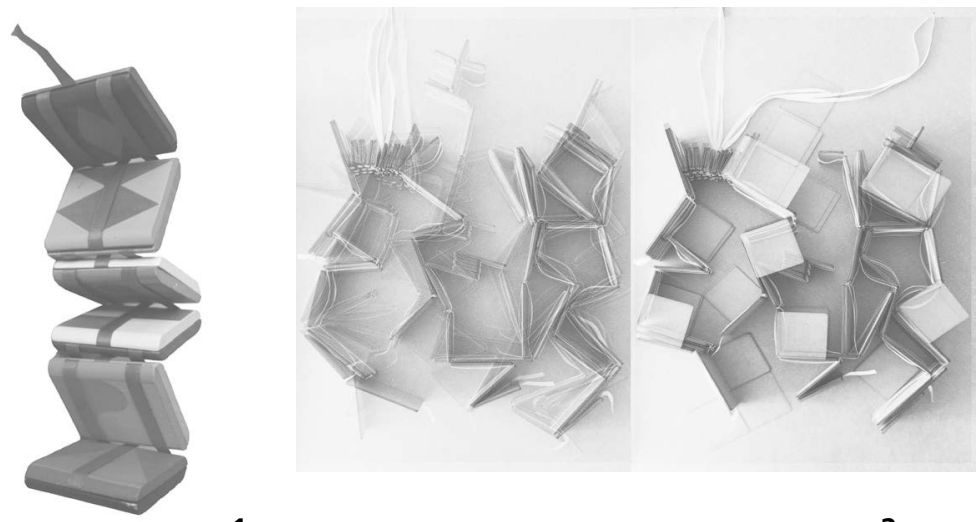

benutzt hat, um das Zeichenprojekt zu kreieren: Er enthüllt einen Teil seines operativen Diagramms. In Stjernfelts Konzeption kann das Diagramm das Gleiche tun, wie Tschumis ,device‘: Es kann „Gedankenexperimente“"2I formell vorantreiben und uns erlauben, in unseren Untersuchungen voranzukommen und mehr zu lernen. ${ }^{22}$

I. Serie: Skizze eines Diagramms Die Abbildungen (I-3) zeigen ein Spielzeug, das aus quadratischen Plättchen besteht, die auf eine besondere Weise zusammengeflochten sind. Dabei wird es ermöglicht, das Spielzeug auf eine andere Weise zu falten, als es der Fall wäre, wenn die Plättchen mit den Nachbarn über Scharniere, die sich um die eigene Achse drehen, verbunden wären. Die Flechttechnik bringt eine Kausalität und Bestimmung mit sich und ermöglicht so die Bewegungsrichtungen des Spielzeugs.

Wird das Diagramm des Spielzeugs in eine architektonische Zeichnung adaptiert, bedeutet dies, das Spielzeug so zu betrachten, als bestände es zum Beispiel aus einer Reihe flexibler Wände. Die Herausforderung, die möglichen Bewegungen zu zeichnen, macht den Zeichenprozess generativ, da es viele Möglichkeiten gibt, sie zu zeichnen. Die Modellfotos und Zeichnungen visualisieren die Bewegungsmuster des Spielzeugs auf zwei- sowie dreidimensionale Weise.

Analoge Maschinen: Das Diagramm bei Deleuze Peirces Konzept des Diagramms ist - zumindest Stjernfelt zufolge - limitiert, indem das Diagramm nicht logisch ,inkonsistent“23 sein kann (trotz der 
1 Fotografie des Spielzeugs (I Serie)

2 Im Modell wird das Spielzeug zu einer flexiblen Struktur. (I Serie)

3 Skizze des Diagramms von der Vielfältigkeit der flexiblen Struktur (I Serie)

Tatsache, dass es gemäß intuitivem Denken entstehen kann). ${ }^{24}$ Peirce sieht das Diagramm als Zeichen, das sich vor allem mit logischem Denken befasst. Doch im Rahmen dieses Beitrags, in dem es auch um ein Verständnis vom konventionellen architektonischen Zeichnen als ein künstlerisches Mittel geht, muss das Konzept von Diagrammen erweitert werden und ein Verständnis für künstlerische Schöpfungen als Prozesse diagrammatischen Denkens einbezogen werden. So geht auch Malene Busk vor, wenn sie Peirces und Deleuzes Diagramme mit gegenseitigem Bezug interpretiert. ${ }^{25}$

Dabei verweist Busk auf zwei Diagrammkonzepte von Deleuze: Die ,,abstrakte Maschine“ ${ }^{26}$ und das „Diagramm als Motiv“. ${ }^{27}$ Laut Busk führt Deleuze Peirces Diagramm ins Extreme. ${ }^{28}$ Obwohl bei Deleuze die zwei Typen von Diagrammen ebenfalls als maschinenähnlich und als unsichtbare Organisatoren von Relationen gelten - ähnlich der ,Denkfigur“ bei Stjernfelt und Peirce - prägt Deleuze das Konzept „Diagramm als Motiv“ in Relation zu der Malweise des Künstlers Francis Bacon. Deleuze argumentiert, dass ein Diagramm eines Künstlers auf einer speziellen ,analogen 
Sprache“29 basiert. Diese Sprache äußert sich durch Züge des Malers, beruhend auf Sinneseindrücken und durch die direkte sinnliche Wirkungskraft zwischen Maler und Gemälde. ${ }^{30}$

Ein solches Diagramm muss, anders als ein logisches Diagramm, nicht unbedingt deutlich gemacht werden, und zeigt sich selbst eventuell nur indirekt in einem materiellen Artefakt, wie zum Beispiel in einem Gemälde. Busk erklärt dies so, dass ein Diagramm, das so verstanden wird, eine ,mentale und mobile Karte“ eines Malers ist beziehungsweise das ,wahre Objekt, welches nicht ein ,Objekt“ ist, sondern Relationen und Veränderungen in Relationen“. ${ }^{\text {I }}$ Das Diagramm als Motiv orchestriert das Material des Gemäldes während seiner Entstehung. Busk hebt das kreative, erkenntnistheoretische Potenzial von Diagrammen in der Herstellung von Gemälden hervor und nennt Bacons Diagramm die „Bacon Maschine“32 - eine Maschine, die durch Bacons bestimmte Denkfigur und seine bestimmte Weise charakterisiert ist. Busk erklärt, welche Rolle Diagramme bei der Erfindung „des Neuen“ haben, indem sie sagt: „The diagram is real to the extent that it participates as a condition of reality in the birth of connections that are still unreal, such as a picturesque or musical composition, a scientific system of functions, references and experiments, or dynamic self-productive economic exchange relationships."33

Wenn das Diagramm dabei eine Rolle spielt, neue Verbindungen und Relationen hervorzubringen, dann ist es dieselbe Rolle, die auch dem Skizzieren in der Architektur häufig zukommt. Es ist interessant, dass Skizzen und Diagramme ein sich überschneidendes epistemologisches Potenzial zu haben scheinen, wenn wir das architektonische Zeichnen im heutigen Kontext digitaler Medien diskutieren, zumal manchmal argumentiert wird, dass nur analoge Handzeichnungen dafür geeignet sind, vage, unbestimmte Ideen hervorzubringen. ${ }^{34}$ Dies wird durch die enge und direkte Verbindung zwischen Denken und Gestalten erleichtert, die das Skizzieren ermöglicht. Auf der anderen Seite ist das Diagramm manchmal eng verbunden mit dem computerbasierten Entwerfen mit ,parametrischen Diagrammen“. ${ }^{35}$ Ich möchte mich jedoch auf den Bereich konzentrieren, den das Skizzieren mit den Diagrammen als kreative, epistemologische Mittel für das Denken und Machen teilt, wobei es nicht wirklich darauf ankommt, ob die Arbeitsgeräte, die bei der Herstellung benutzt wurden, analog oder digital waren. Schließlich kann ein Diagramm - wie ich es im Folgenden 


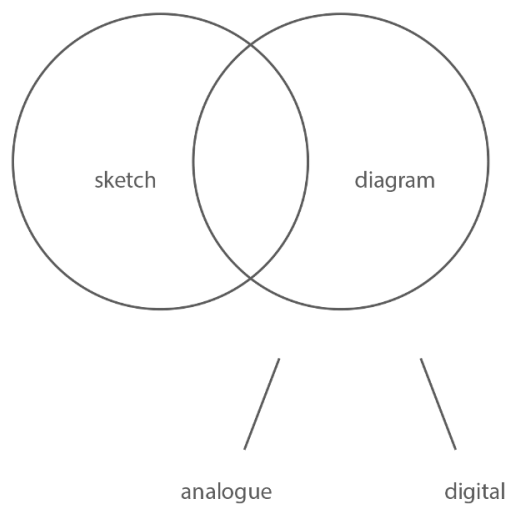

4 Theoretisches Diagramm

mit Hilfe von Nelson Goodmans Notationstheorie aufzeigen werde - sowohl analog als auch digital sein. Architekturzeichnungen als Skizzendiagramme zu verstehen, erlaubt es uns, sie sowohl als Medium als auch als Artefakt zu verstehen. Zum einen im Umgang mit logischem und intuitivem Denken, zum anderen mit analoger und digitaler Notation. In beiden Fällen, in manuell sowie in computerbasiert ausgeführten Diagrammen, geht es um eine interaktive und iterative Struktur: Ein generativer Dialog schwankend zwischen Handeln, Ausführen, Beobachten, Verändern, Denken und erneutem Handeln. Deshalb behaupte ich, dass sich diagrammatisches Denken in architektonischen Designprozessen nicht grundlegend verändert hat, auch wenn sich die Notationsformen und die Arbeitsgeräte der Architekten verändert haben (Abb. 4).

II. Serie: Skizze eines analogen Diagramms Diese Serie von Zeichnungen (Abb. 5-8) strebt ein analoges Diagramm an. Analog in dem Sinne, dass das Motiv, das sich durch die Serie fortsetzt, von Hand gezeichnet ist. Das Diagramm, das genutzt wurde, um die Serie zu generieren, war das Spiel „Memory“. Wenn man Memory als Diagramm betrachtet und an die Zeichnung heranführt, dann generiert das Spiel kontinuierlich verändernde Zeichnungen: Quadratische Plättchen mit einem Motiv auf einer Seite werden von den Spielern abwechselnd umgedreht, um zusammengehörige Motive zu finden. In dieser Serie von Zeichnungen passen die Motive nicht zueinander, wodurch der Sinn des Spiels zerstört 
wird, sodass sich das Zeichenspiel nicht basierend auf einer Logik, sondern auf subjektiven Zügen entwickelt.

Digitale Diagramme bei Nelson Goodman Die analoge Sprache bei Deleuze ist verschieden von der analogen Notation in Nelson Goodmans Notationstheorie und dennoch haben beide mit Diagrammen zu tun. Allerdings können Diagramme bei Goodman sowohl analog als auch digital sein. ${ }^{36}$ Goodman fasst das Digitale als allografische Notationsverfahren zusammen, wobei das Analoge als autografische Verfahren, die streng genommen keine Notationen sind, zusammengefasst wird. Das Digitale ist allografisch, wobei ein allografisches Kunstwerk dadurch charakterisiert ist, dass es von seinem Autor oder seiner Quelle unabhängig ist. Konventionelle Musiknoten sind Goodmans bestes Beispiel einer digitalen Notation in den Künsten, die Anweisungen mit Hilfe einer konventionalisierten und unmissverständlichen Notation weitergeben, die jeder versteht, der die Konventionen erlernt hat. ${ }^{37}$ Ein autografisches Kunstwerk ist auf der anderen Seite durch eine enge Verbindung zu dessen Autor charakterisiert, so wie die Hand eines Malers mit einem Pinsel Spuren mit unterschiedlichem Druck auf einer Leinwand hinterlässt. Analoge Notationen, die jedoch streng genommen für Goodman keine Notationen sind, sind ,dicht“ und „durchweg undifferenziert“ ${ }^{38}$ so wie ein Pinselstrich gemalt auf Papier.

Analoge Notationen übermitteln oder übersetzen nichts per se; vielmehr sind sie selbst Sachen wie ein Gemälde. In diesem Sinne werden autografische Kunstformen nicht übersetzt, es braucht kein Medium zwischen Autor und Werk, um das Werk zu vermitteln, im Gegenteil zu digitalen und allografischen Kunstformen. Musiknoten sind ein Medium ${ }^{39}$ zwischen einem Schöpfer und einer Aufführung in einer Weise, die sich davon unterscheidet, wie Pinsel oder Stifte zwischen Schöpfer und Gemälde eingesetzt werden. Die Verbindung zwischen einem Architekten und einer Zeichnung ist eine enge - so wie Maler und Malerinnen arbeiten Architekten und Architektinnen direkt mit der Zeichnung. Eine Zeichnung kann jedoch auch Anweisungen durch konventionelle Notationsweisen übermitteln, wodurch sie über sich selbst hinausreicht und zum Medium wird, wie es ein Gemälde nicht tut. Ein Grundriss zum Beispiel übermittelt Anweisungen besser, ähnlich wie Musiknoten. Natürlich hat ein Gemälde auch einen Effekt über sich selbst 


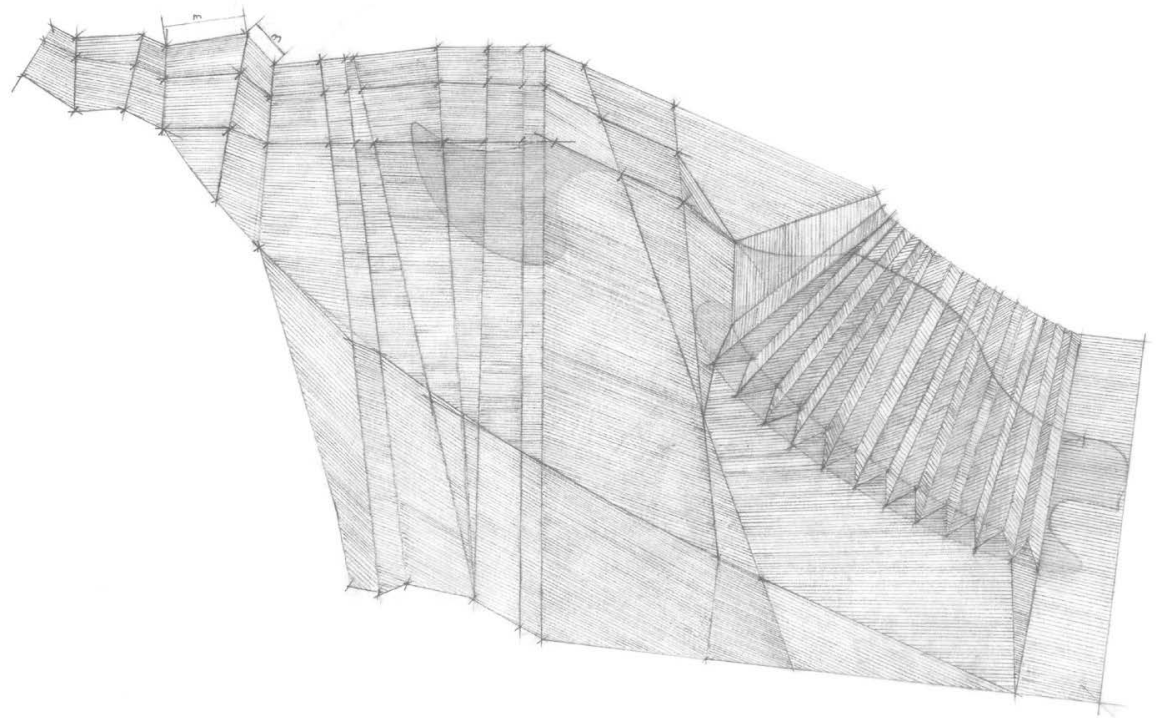

5 Erste Zeichnung des analogen Diagramms (II Serie)

hinaus; doch gibt es normalerweise keine Anweisungen, wie etwas mit anderer Materialität zusammengeführt oder aufgeführt werden soll. Ein Grundriss ist außerdem bildähnlich, selbst ein Artefakt mit Bezug zum Autor, aber auch ein Medium, das konventionalisierte Anweisungen vermitteln kann. Deshalb teilen konventionelle Grundrisse Eigenschaften sowohl mit Gemälden als auch mit Musiknoten.

Die unmissverständliche Art und Weise, wie ein Bauplan über sich selbst hinausreichen kann, ist in Goodmans Notationstheorie charakteristisch für digitale Notationen, die dort eher Mittel als Zweck sind; und dies gilt, egal welches technische Equipment bei der Herstellung der Notation verwendet wurde. Dies ist auch der Grund, warum es für Goodman möglich war zu sagen, dass konventionelle, orthogonale Pläne - insbesondere Grundrisse „digitale Diagramme“ sind, ${ }^{40}$ schon bevor moderne Computer existierten. Konventionelle Grundrisse sind digital, weil ihre Notationsweise klare Anweisungen übermittelt, auf Gebäude verweist und unmissverständlich mit einem Gebäude übereinstimmen kann. Weil Baupläne so wichtige Bestandteile der Arbeitsmittel der Architekten sind, neigt Goodman dazu, den Schluss zu ziehen, dass die 

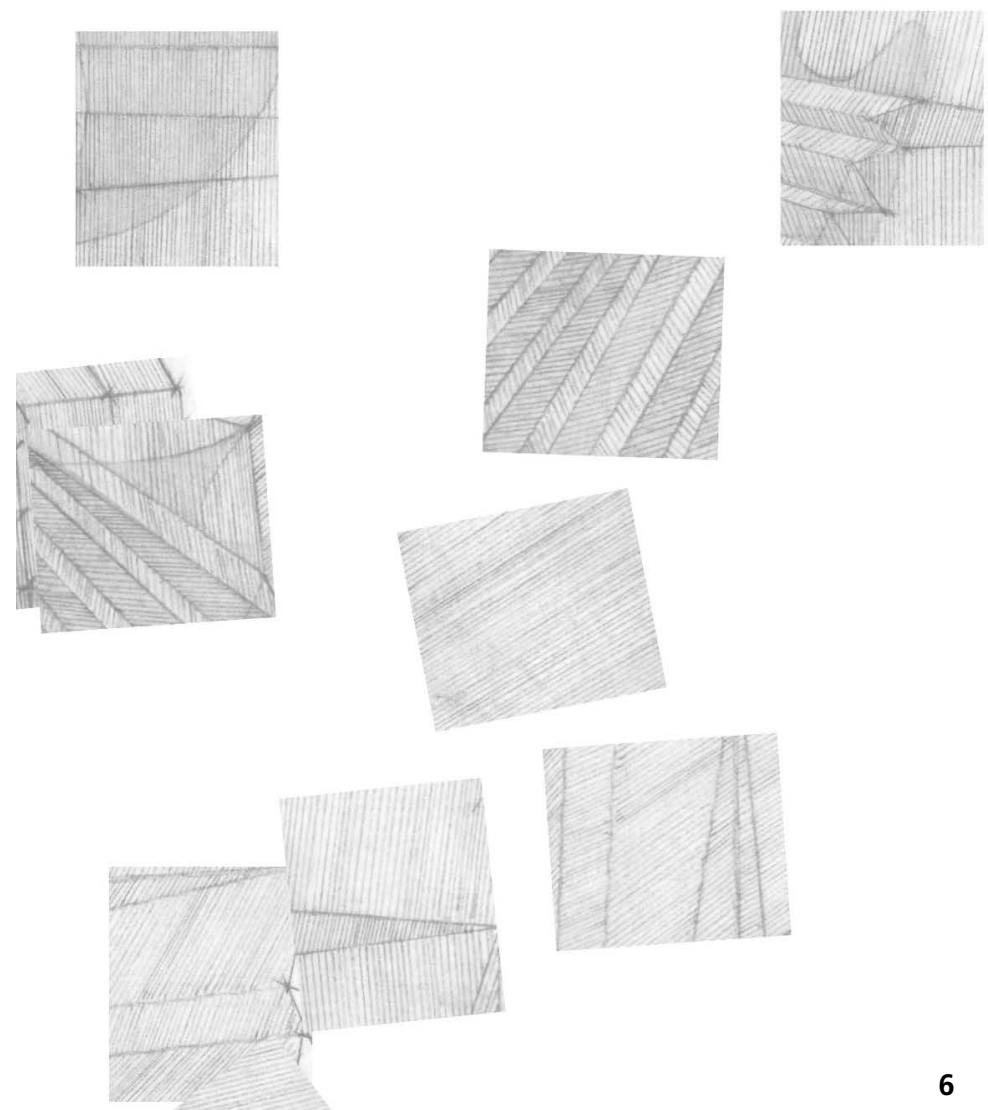

6 Ausschnitt des analogen Diagramms. Die erste Zeichnung wird der Generativität des Spiels Memory ausgesetzt.

(II Serie)

7 Dritte Skizze des analogen Diagramms. Die Generativität der Zeichnung muss nicht den Spielregeln folgen. Daraus entstehen unterschiedliche Konfigurationen der Zeichnung. (II Serie)

Architektur in Bezug auf Notationen eine allografische und digitale Kunstform ist. Doch kann er dabei auch nicht die analogen Aspekte konventioneller Zeichnungen ignorieren, ${ }^{4 \mathrm{I}}$ wie deren bildähnli- 


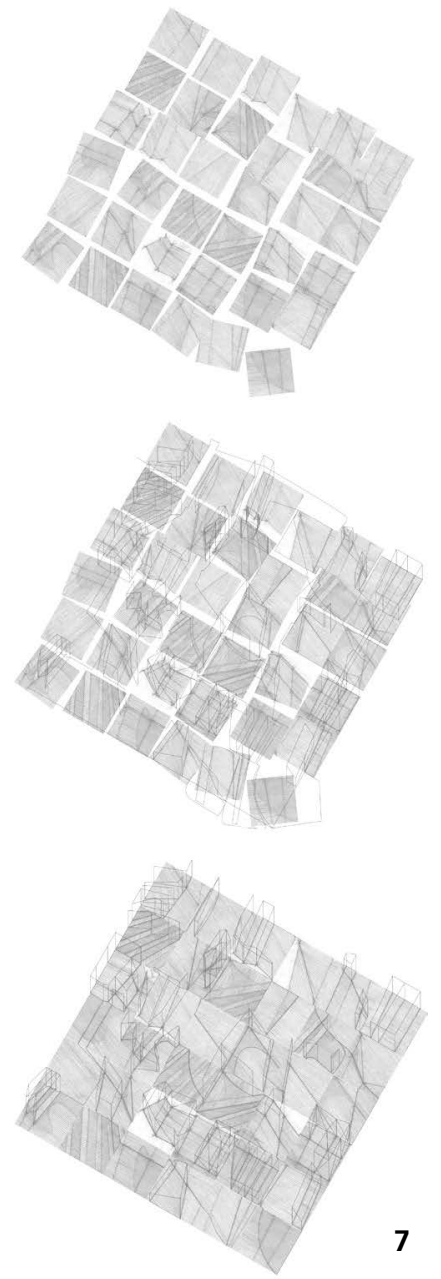

chen und ikonischen Qualitäten oder die Unbestimmtheit des Skizzierens. Auch kann er nicht die Tatsache ausblenden, dass selbst fertige Baupläne autografische, analoge Elemente aufweisen, so wie lokale oder materialspezifische Angaben. Somit werden Pläne von Goodman nur ohne material- oder ortspezifische Angaben als ,digitale Diagramme“ definiert. ${ }^{22}$ Schließlich entscheidet sich Goodman dafür, dass die Architektur ein gemischter Fall beziehungsweise Übergangsfall ist und sowohl analoge als auch digitale Notationen in enger Verbindung verwendet. ${ }^{43}$

Goodman denkt, dass die Architektur aufgrund einer Unreife der architektonischen Notationstechniken den Status des Digitalen noch 

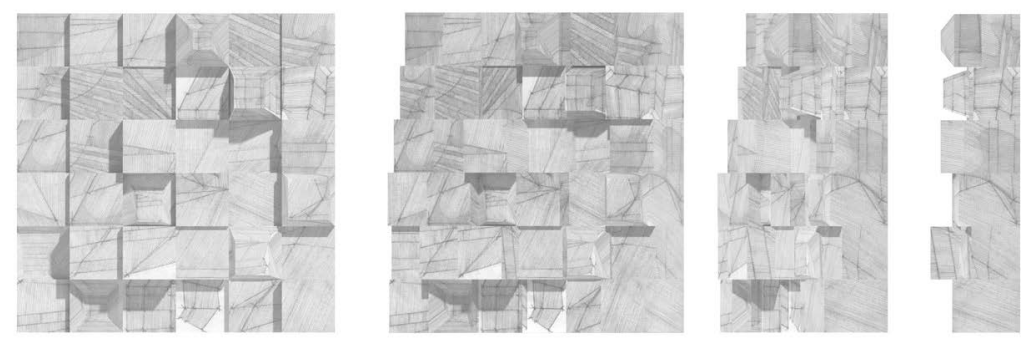

8 Unterschiedliche Konfigurationen des analogen Diagramms (II Serie)

nicht vollkommen erreicht hat. ${ }^{44}$ Ich denke allerdings, dass Architektur, wenn Architekten und Architektinnen - vor allem heute mit digitalen Arbeitsgeräten entwerfen können, weiterhin ein Übergangsfall ist, indem sowohl analoge als auch digitale Aspekte des Diagramms für das Schaffen und Denken genutzt werden. Außerdem sind analoge und digitale Notationsformen aufgrund der Werkzeuge und des technischen Equipments im Herstellungsprozess nicht so definiert, dass sie entweder ausschließlich analog oder ausschließlich digital sind. Goodman hat auch betont, dass eine Notation abhängig von der ,Lesart" 45 sowie von den Eigenschaften der Vorgänge, die in der Notation ausgedrückt werden, entweder analog oder digital ist. Dies gilt zum Beispiel für eine statistische Kurve, die im Prinzip in Verbindung mit allem als digital betrachtet werden kann, während eine Kurve, die aufgrund eines singulären Events in einem geschlossenen Kontinuum - wie einem Erdbeben entsteht, analog ist. Dies bedeutet, dass es möglich ist, eine digitale Notation per Hand zu erstellen, genauso wie es möglich ist, eine analoge Notation mit dem Computer zu erstellen. Dies bedeutet aber auch, dass eine konventionelle Architekturzeichnung, die üblicherweise mit analogen Werkzeugen angefertigt wird, bereits durch eine digitale Notation übertragen wird.

Da ich eine diagrammatische Ebene in der architektonischen Zeichnung verfolge, muss angemerkt werden, dass sich orthogonale Projektionen, die als eine der am meisten verwendeten Architekturzeichnungstechniken typisch sind, mühelos in verschiedene Computerprogramme eingefunden haben. Architektinnen und Architekten ,,sehen“ durch orthogonale Projektionen, die konventionell dem Zeichnen angehören. Doch wie der Architekt und Archi- 
tekturtheoretiker Robin Evans erläutert, ist projizierende Geometrie zum Sehen nur eine von drei Geometrien, die die Zeichnung als architektonisches Arbeitsmedium ausmachen. Evans ist einer von wenigen Theoretikern, die begründen, dass Geometrie und durch Zeichnungen vermittelte Projektionen gemeinsam die gebaute Architektur sowohl kausal als auch willkürlich mitgestalten. ${ }^{6}$ Dies hat damit zu tun, dass architektonisches Zeichnen nicht „bloß Geometrie" ist, sondern dass Geometrie als Medium verwendet wird. ${ }^{47}$ Dieses Medium beruht auf vielen ,übereinander“ gelegten Geometrien: Projizierende Geometrie zum Sehen, beschreibende Geometrie zum Messen und Zusammensetzen und zuletzt ,signified" Geometrie ${ }^{48}$ - ein Konzept, das von Evans kreiert wurde -, die umschreibt, wie Geometrie manchmal auf expressive Weise verwendet wird.

Mit Evans Darstellung der Architekturzeichnung wird zum einen deutlich, dass mit der Digitalisierung eine der Geometrien, nämlich die projizierende Geometrie zum Sehen, ungezwungen in Zeichen- und Entwurfsprozesse eingezogen ist. Zum anderen wird verdeutlicht, dass projizierende Geometrie diagrammatisch ist, indem sie mit analogen und digitalen Werkzeugen zusammenarbeiten kann. Dies bedeutet auch, dass die projizierende Geometrie zum Sehen von der Geometrie zum Machen und Beschreiben getrennt wurde. Geometrische Zeichenwerkzeuge wurden ursprünglich genutzt, um sowohl die projizierende Geometrie (zum Sehen) als auch die beschreibende Geometrie (zum Machen) zu unterstützen. In Computerprogrammen blicken Architekten und Architektinnen jedoch auf Geometrien, die auf neue Weisen computerbasiert generiert werden können, basierend auf traditionellen orthogonalen, isometrischen und perspektivischen Geometrien. Dies ist eine Mutation, bei der projizierende Geometrie das konventionalisierte Zeichnen als eine ikonische Weise, Architektur $\mathrm{zu}$ lesen und zu betrachten, am Leben hält. In diesem mutierten Zustand vermischen sich Teile konventioneller Zeichnungen mit computerbasierten Leistungen, wobei sich konventionelle Abhängigkeiten zwischen technischem Equipment und Notationsformen ändern. Die Unbestimmtheit, die im Entwurfsprozess für die Entstehung von etwas Neuem sehr wichtig ist, wird dadurch versetzt. Sie entfällt aber nicht vollständig, sondern entfaltet sich an anderer Stelle. Deswegen mag das Skizzieren andere Konzepte benötigen und in anderen Bereichen des Entwurfsprozesses vorkommen. 


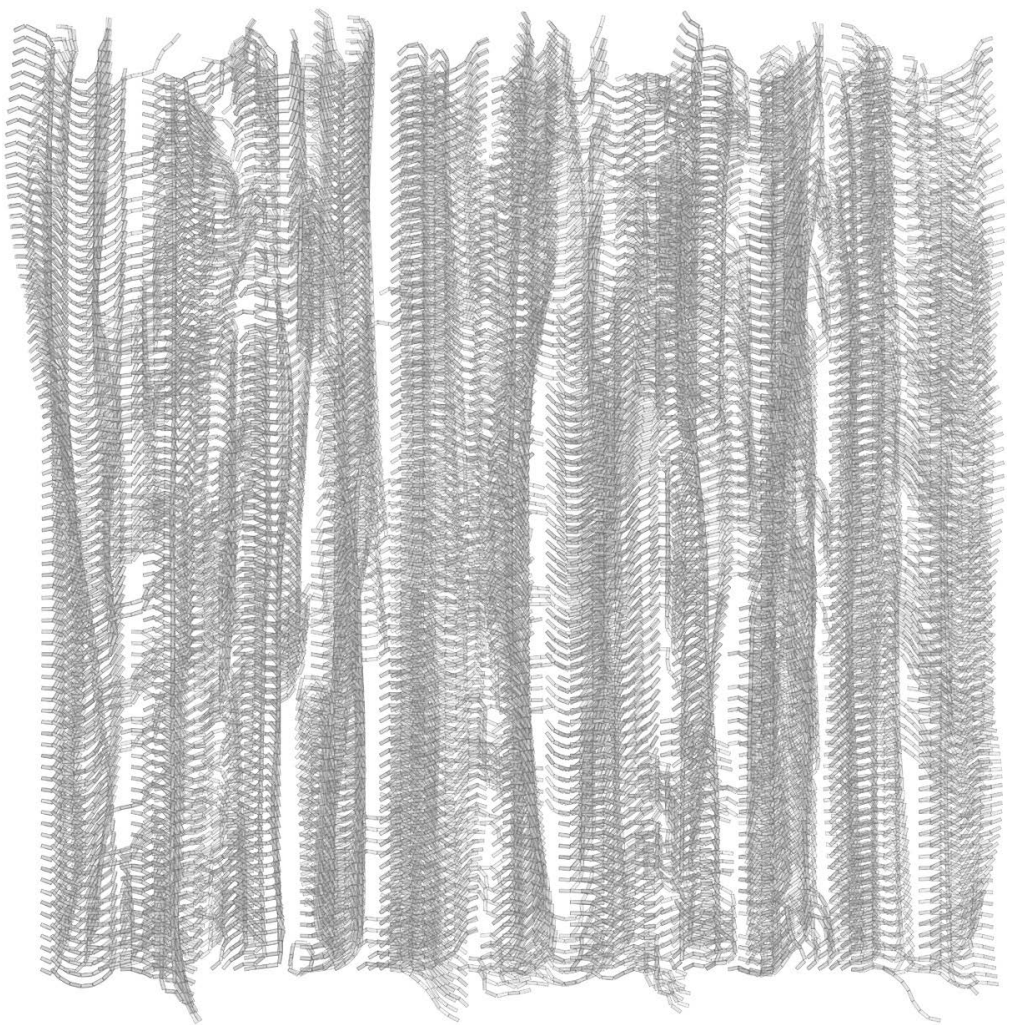

III. Serie: Skizze eines digitalen Diagramms Manche behaupten, dass Computer zum Skizzieren nicht geeignet sind und dass man im Voraus wissen muss, für was der Computer im Entwurfsprozess verwendet werden soll. Doch im Gegensatz dazu können erfahrene Programmierer Skripte so reibungslos schreiben, dass es so wirkt, als würden sie skizzieren. Das Skizzieren der folgenden Zeichnungen erfolgte, als der erfahrene Programmierer Abe Pazos und ich das Verhalten der bereits erwähnten Spielzeugschlange simulieren wollten. Das Ziel war, ein Skript zu entwickeln, das die Bewegung der Schlange simulieren kann. Doch auf dem Weg, dies zu erreichen, hat sich der Prozess verzweigt, was zur Serie dieser Zeichnungen geführt hat (Abb. 9-I2), bei der das digitale Skript unterschiedliche geometrische Figuren generierte. Wir haben verschiedene Varianten des Skripts erstellt, die interpretati- 


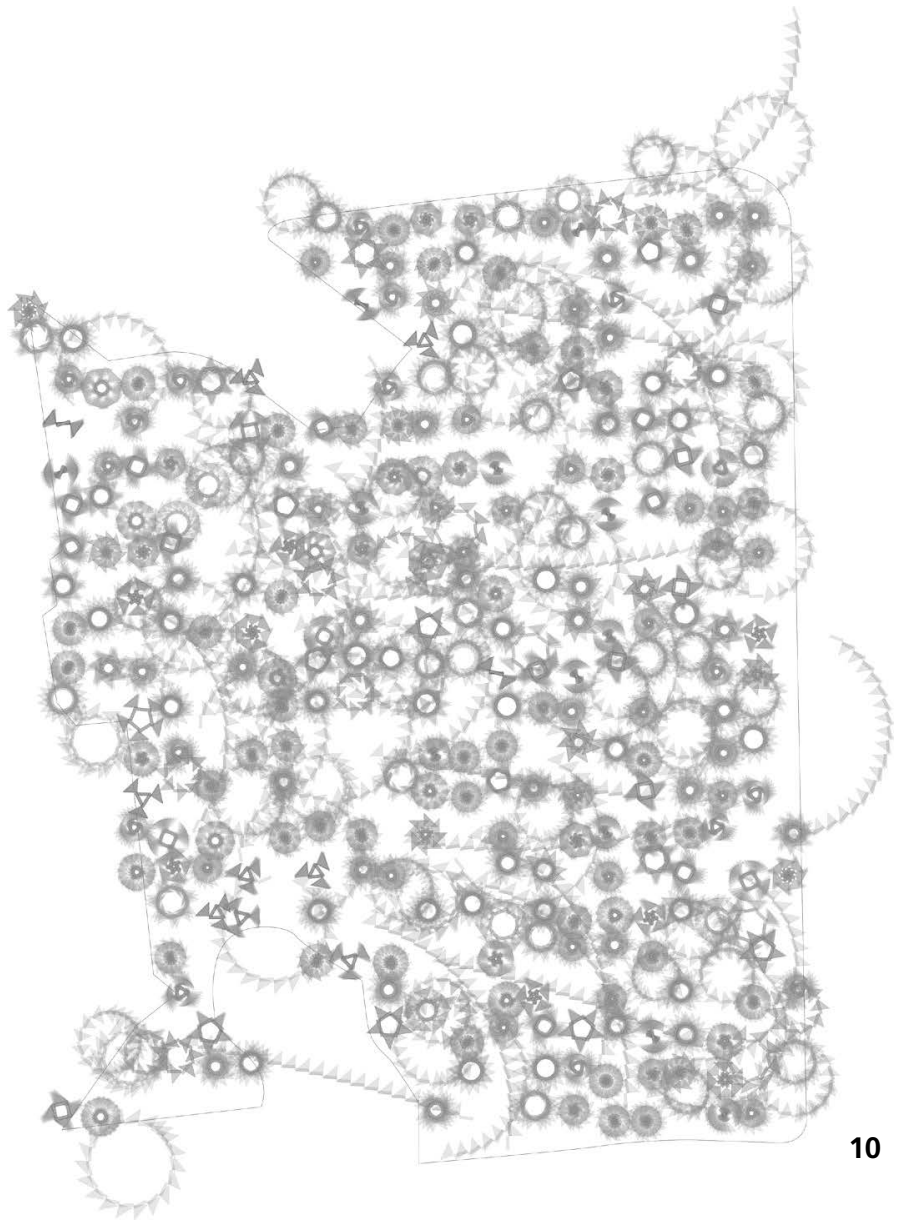

9 Skizze eines digitalen Diagramms. Annäherung an eine algorithmische Simulation des Spielzeugs (III Serie)

10 Skizze eines digitalen Diagramms (III Serie)

onsoffene Zeichnungen und somit gewissermaßen Skizzen erzeugt haben, die man weiterentwickeln könnte: manche, die wie tanzende Kleider von oben aussehen, solche, die wie verdrehte Flügel von Vögeln erscheinen, und schließlich jene, die wie Sterne oder Stufen in der Draufsicht wirken. 


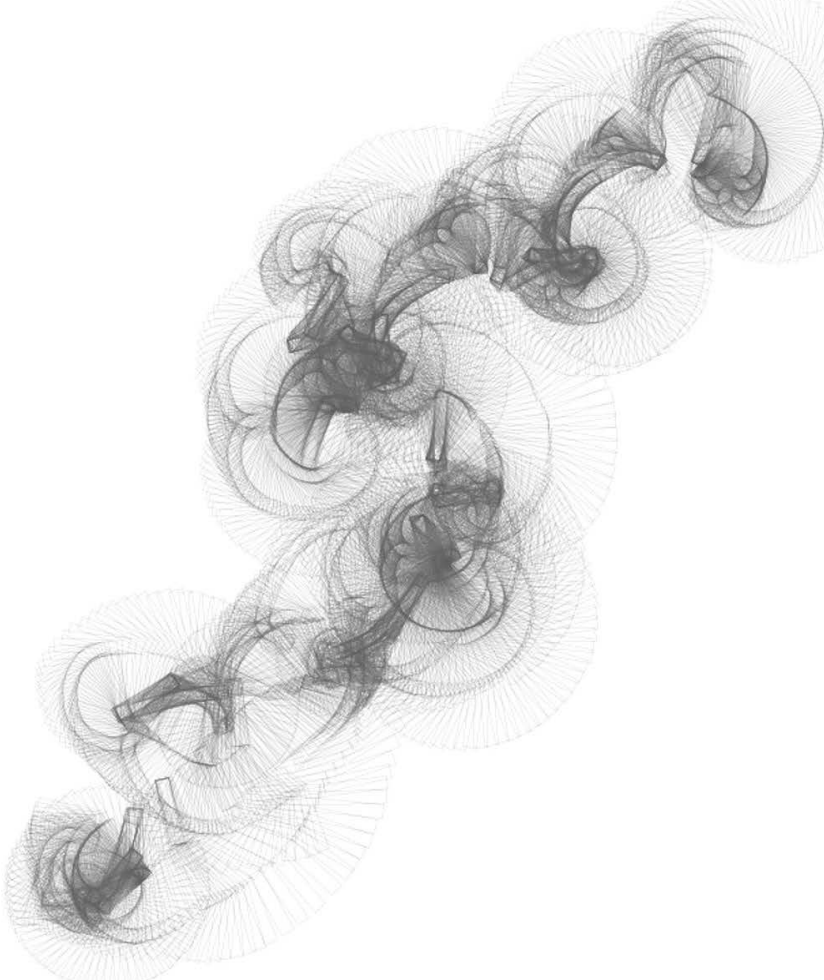

Konklusion Goodmans Argument, dass konventionelle Architekturzeichnungen nie vollständig analog oder vollständig digital waren, wurde hier aufgegriffen, und dahingehend angepasst, dass konventionelle Zeichnungen immer gemischte Übergangsdiagramme waren und weiterhin sind. Es wurde ausgeführt, dass zeichnerisches Denken diagrammatisch ist, also sowohl mit Unbestimmtheit und Empfindungen wie auch mit komplett konsistentem, logischem Denken arbeitet. Des Weiteren wurde Goodmans Argument aufgegriffen, dass Diagramme sowohl analog als auch digital sein können.

In der Architektur, in der die Bandbreite an Gestaltungsmöglichkeiten aufgrund von sich weiterentwickelnden Computertechnologien zunimmt, wurde das Skizzieren mit Diagrammen als ein Medium eingeführt, das menschliches Denken mit analogen und digitalen Notationsformen in bestimmten subjektiven Entwurfspraktiken verbindet. Außerdem wurde aufgezeigt, dass die Generativität, die häufig mit dem Skizzieren per Hand in Verbindung 


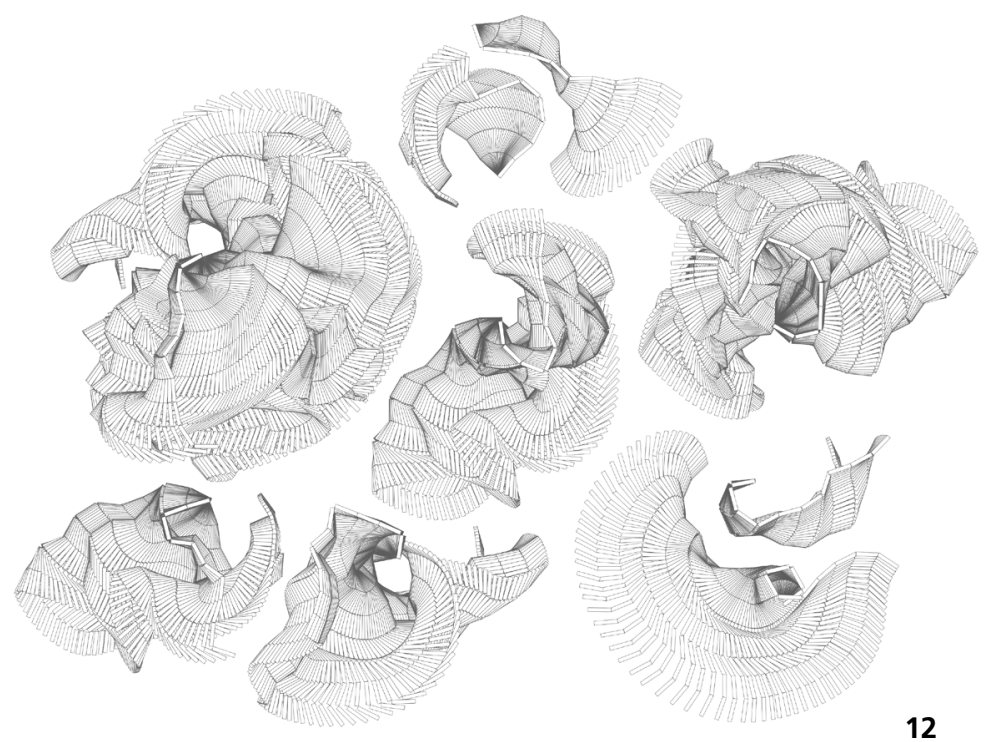

11,12 Digitale Diagramme (III Serie)

gebracht wird, ebenfalls ein Merkmal von Diagrammen ist, egal ob analog, digital oder gemischt. Folglich wurde das Konzept eines Skizzendiagramms beschrieben, das einen generativen Weg des Denkens über und Schaffens von Architektur bildet und nicht nur von technischen Arbeitsgeräten bestimmt wird. Meine Zeichnungen waren eine treibende Kraft hinter diesem Konzept und wurden parallel dazu gestaltet: handgemachte Computerzeichnungen, die sich zwischen digitalen und analogen Notationsformen verflechten, zwischen konventionellen Zeichentechniken und computerbasierten Verfahren.

\section{Anmerkungen}

1 Diese ist eine revidierte Ausgabe des Artikels "Sketch Diagrams", ursprünglich herausgegeben in der Anthologie Refractions. Anna Katrine Hougaard, "Sketch Diagrams”, in Anna Katrine Hougaard, Martin Søberg, Kristine Annabell Torp u. a. (Hg.), Refractions. Artistic Research in Architecture, Kopenhagen 2016, S. 137-167. Ins Deutsche übersetzt von Julian Franke. 
2 Frederik Stjernfelt, Diagrammatology. An Investigation on the Borderlines of Phenomenology, Ontology, and Semiotics, London, New York, Dordrecht u.a. 2011, S. 114.

3 Douglas R. Anderson, "The Evolution of Peirce's Concept of Abduction", Transactions of the Charles S. Peirce Society 22, 1986, S. 150.

4 Charles S. Peirce, Semiotik og Pragmatisme, Kopenhagen 1994, S. 20.

5 Ebd., S. 98-101.

6 Ebd., S. 100f., 118-121.

7 Stjernfelt, Diagrammatology (Anm. 2), S. 128.

8 Peirce, Semiotik (Anm. 4), S. XX.

9 Stjernfelt, Diagrammatology (Anm. 2), S. 128. Im Original: "skeleton-like sketch of relations", Übersetzung hier von Julian Franke und Anna Hougaard.

10 Ebd., S. 105.

11 Nicolaus Gansterer (Hg.), Drawing a Hypothesis. Figures of Thought, Wien, New York, 2011, S. 22 und S. 154-173.

12 Siehe Stjernfelts „epistemological device“. Stjernfelt, Diagrammatology (Anm. 2), S. 114.

13 Siehe auch die Distinktion der Philosophin und Architektin Sabine Ammons zwischen „Wissen-im-Werden" und „Wissen-als-Ergebnis". Sabine Ammon, „Wie Architektur entsteht. Entwerfen als epistemische Praxis" in: Sabine Ammon und Eva Maria Froschauer (Hg.), Wissenschaft Entwerfen, München 2013, S. 354f.

14 Bernard Tschumi, The Manhattan Transcripts, London 1994.

15 Bernard Tschumi, Architecture Concepts. Red is Not a Color, New York 2012, S. $24 f$.

16 Tschumi, Transcripts, (Anm. 14), S. XXIIf.

17 Ebd., S. 6.

18 Ebd., S. XXV. Nach Wissen der Übersetzer wurde der Begriff, der "Mittel", "Medium”, „Apparat", „Vorrichtung" bedeutet, nicht ins Deutsche übersetzt.

19 Ebd., S. 12.

20 Tschumi, Concepts (Anm. 15), S. 108.

21 Stjernfelt, Diagrammatology (Anm. 2), S. 99.

22 Ebd., S. 49-88.

23 Ebd., S. 98.

24 Ebd., S. 114. 
25 Malene Busk, "The Diagrams of Peirce and Deleuze", in: Cort Ross Dinesen (Hg.), Cartography, Morphology, Topology, Kopenhagen 2009, S.170-173.

26 Gilles Deleuze and Félix Guattari, A Thousand Plateaus. Capitalism and Schizophrenia, London 2004, S. 156.

27 Gilles Deleuze, Francis Bacon - Logik der Sensation, Paderborn 2002, S. 97-105.

28 Busk, The Diagrams of Peirce and Deleuze (Anm. 25), S. 170.

29 Deleuze, Francis Bacon (Anm. 26), S. 99.

30 Ebd., S. 98.

31 Busk, "The Diagrams of Peirce and Deleuze" (Anm. 25), S. 170. Übersetzt von Julian Franke.

32 Ebd., S. 172.

33 Ebd.

34 Vgl. z.B. Michael Graves, "Architecture and the Lost Art of Drawing", The New York Times September 2, 2012.

35 Patrik Schumacher, „Parametric Diagrams”, in: Marc Garcia (Hg.), The Diagrams of Architecture, West Sussex 2010, S. 263.

36 Nelson Goodman, Languages of Art, Indianapolis, 1976, S. 170.

37 Ebd., S. 161.

38 Ebd., S. 121 und 160.

39 Ein Medium ist ein vermittelndes Element, http://www.duden.de/ rechtschreibung/Medium_Vermittler_Traeger [27.3.2019].

40 Goodman, Languages (Anm. 36), S. 219-221.

41 Ebd., S. 221.

42 Ebd., S. 120, $192 f$.

43 Ebd., S. 219.

44 Ebd., S. 221.

45 Ebd., S. 170.

46 Robin Evans, The Projective Cast - Architecture and Its Three Geometries, Cambridge, MA und London, 1995, S. 228.

47 Ebd., S. XXVf. und $37 f$.

48 Ebd., S. 349. Teile von Robin Evans Texten von The Projective Cast wurden in Arch+ 137, 1997 ins Deutsche übersetzt, jedoch nicht dieser Begriff. 


\section{Fotografie und Entwerfen}

Fotografie und Architektur Als Mitte der I990er Jahre die Digitalisierung der Amateurfotografie ihren Siegeszug antrat, wurde in medientheoretischen Diskussionen schon bald auf ein bevorstehendes ,Ende des fotografischen Zeitalters“" spekuliert. Die indexikalische Beweiskraft der Fotografie, der zufolge ein fotografisch belegtes Ereignis auch sicher stattgefunden hat, schien ihre Gültigkeit zu verlieren, weil durch die Möglichkeit der algorithmischen Ansteuerung jedes Bildpunktes mit dem Computer, die Manipulation von Bildinhalten eine neue Qualität erlangt hatte. Zudem reihte die Digitalisierung des Fotos dieses in die allgemeine Kategorie der „Computergrafik“ ein, so dass zwischen einem Digitalfoto und einer vollkommen simulierten Bildlichkeit kaum mehr eine Differenz zu bestehen schien. Doch ein ,postfotografisches Zeitalter"22, so kann heute konstatiert werden, hat sich partout nicht einstellen wollen - im Gegenteil, die Digitalisierung der Fotografie hat bis heute einen ungeheuren Anstieg der Produktion und des Konsums fotografischer Abbildungen nach sich gezogen. Umweltrezeption erfolgt heute mehr denn je durch Bilder. Auf Displays von Smartphones, auf Computermonitoren, in Film und Fernsehen - in nahezu allen Lebensbereichen kann eine steigende Relevanz bildlicher Darstellungen beobachtet werden. Seitdem Mobiltelefon und digitale Fototechnik im Smartphone verschmolzen sind, wird nahezu alles mit Kameras aufgenommen und viele Ereignisse werden überhaupt erst als wesentlich wahrgenommen, wenn sie auch fotografiert verfügbar sind. Bereits I980 bescheinigte die US-amerikanische Autorin Susan Sontag den Menschen einen „Zwanghaften Drang“ Erfahrungen nur dann als real erlebt zu empfinden, wenn sie mit der Aufnahme eines Fotos belegt werden. ${ }^{3}$ 
Diese Beschreibung scheint heute zutreffender als jemals zuvor in der Geschichte fotografischer Bildproduktion zu sein. Gleichzeitig gilt sie nicht nur für die fotografische Reproduktion menschlicher Erfahrungen und Aktionen, sondern ist auf Umweltwahrnehmung und -aneignung allgemein übertragbar. Weil gestalterische Arbeit immer aufs engste mit Wahrnehmungsprozessen und mit medialen Aneignungsweisen von Umwelt zusammenhängt, bleibt auch die Konstruktion räumlicher Formationen, also auch die Architekturproduktion, nicht unberührt von der spezifischen Form der Bedeutungsbildung durch Fotokameras. Wechselwirkungen zwischen Bild und Raumgestaltung sind dabei selbstredend keine Phänomene, die erst mit Erfindung der Fotografie entstanden wären. Ein Widerhall bildlicher Logik in baulichen Umsetzungen kann nachgewiesen werden, seit in der Renaissance mit der Perspektive ein systematischer Zusammenhang zwischen Ordnungen des Raumes und Ordnungen der Fläche entwickelt wurde.

Die perspektivische Reduktion der Anschauung von Umwelt auf zwei Dimensionen ist dabei von jeher sowohl für die Repräsentation wie auch für die Produktion von Architektur relevant gewesen. Eine zentrale Rolle bei dieser bidirektional bestehenden Abhängigkeit zwischen Raum und Bild ist - ganz im Sinn des Satzes „The Medium is the Message“4 des Kommunikations- und Medientheoretikers Marshall McLuhan - den hierbei wirksamen medialen Mitteln zuzuschreiben. Wie eine medial geprägte Wechselwirkung zwischen bildlichen Darstellungen und Raumgestaltung sich Ausdruck verleiht, lässt sich anhand eines historischen Beispiels verdeutlichen: Lange vor Erfindung der Fotografie gab es technische Hilfsmittel, mit denen die Rezeption einer Umgebung in linearperspektivisch systematisierter ,Optimalsicht ' ermöglicht wurde. Die Camera obscura ist eine solche Apparatur, deren Bildgebungsverfahren Maler als Werkzeug zur vereinfachten Anfertigung perspektivischer Gemäldevorzeichnungen verwendeten. Ein anderes, von der Bildproduktion unabhängiges Beispiel solcher ,Blickidealisierungs-Apparate“ sind sogenannte Claude-Gläser. Mit Hilfe dieser konvex geformten und getönten Taschenspiegel wurden ab dem I8. Jahrhundert Landschaften ,echtzeitlich' und in der direkten Anschauung, in ähnlich komponierter und malerischer Weise wahrnehmbar gemacht, wie sie aus dem Vorbild stimmungsvoller Landschaftsgemälde des französischen Malers Claude Lorraine aus dem I7. Jahrhundert bekannt waren. Der 
Einfluss solch idealisierter Landschaftsbilder auf die Vorstellung idealer Landschaftserscheinung zog schließlich nicht nur die Erfindung und Anwendung optischer Blickidealisierungs-Apparate wie die Caude-Gläser nach sich, sondern er ging so weit, dass diese Darstellungen zum Vorbild für wirkliche Raumgestaltung wurden. ${ }^{5}$ Die seit dem I8. Jahrhundert zunächst in England und später auf dem europäischen Festland angelegten Landschaftsgärten können als gebaute Gartenbilder im Sinn einer Projektion komponierter Landschaftsgemälde in die Wirklichkeit gelesen werden - als Versuch, das besondere Wesen dieser Bilder physisch zugänglich zu machen, indem ihre verdichtende, bildlich-zusammenfassende Wirkung zu einem in Wirklichkeit erlebbaren Raumereignis materialisiert wurde. Führt man sich die äußerliche Ähnlichkeit der aus unterschiedlichen Epochen stammenden ,Wahrnehmungsapparate Claude-Glas und Smartphone-Kamera vor Augen (Abb. I), dann stellt sich die Frage, ob sich ähnliche, wie für die Claude-Gläser beschriebene Zusammenhänge und Rückwirkungen zwischen Bild, Medium und Wirklichkeit, auch heute in Folge der massenhaften Verwendung von Smartphone-Kameras nachweisen ließen - ob den durch sie verfügbar gemachten fotografischen Bildern ähnliche raumproduzierende Kräfte innewohnen?

Bevor dieser Frage nach den möglichen raumbildenden Potenzialen heutiger Smartphonefotografie nachgegangen wird, soll der Blick zunächst auf herkömmliche perspektivische Darstellungsweisen und ihre bis heute nachweisbare Wirkung auf Bauprojekte gerichtet werden. In der Konzeption des Perspektivbildes der Renaissance lässt sich häufig eine spezifische Differenz zwischen dem nach perspektivischen Regeln konstruierten architektonischen Bildhintergrund und einem sich vor diesem frei entfaltenden, aktiven Bildvordergrund ausmachen. Der amerikanische Architekturtheoretiker Robin Evans spezifiziert diese charakteristische Raumteilung im Bild anhand eines Renaissancegemäldes von Rafael wie folgt: ,[Die Figuren stehen] gleichsam direkt hinter dem Bildrahmen Schlange und lassen die Architektur als bloßen Hintergrundprospekt großenteils leer. Mit dieser Schicht menschlicher Bewegung im Vordergrund und dem teleskopischen Blick auf leere Gebäude im Hintergrund konnte die zu erzählende Geschichte und die Bühne, auf der sie Platz fand, unabhängig voneinander bearbeitet werden."6 


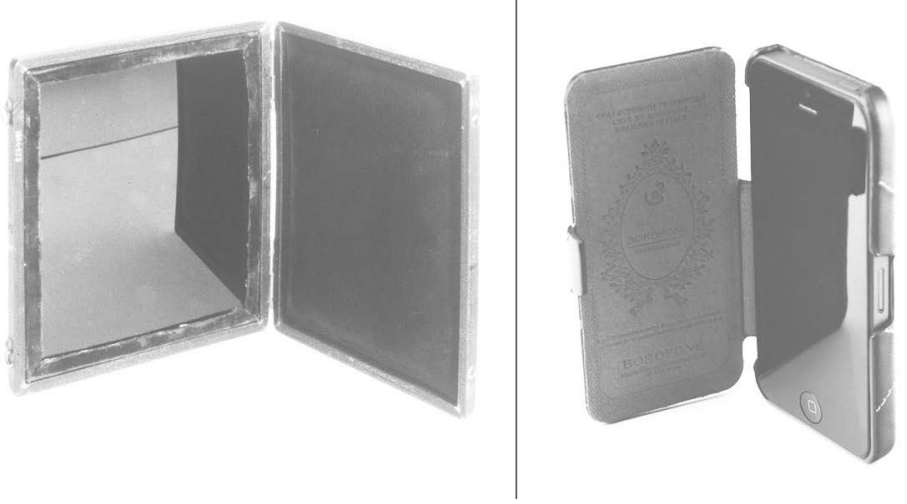

1 Claude-Glas (getönter Taschenspiegel), um 1870 / iPhone 5 in Schutzhülle, 2013

Evans beschreibt hier, wie die eine bestimmte Raumkonstellation und Architektur befördernde Perspektivkonstruktion Albertis in dem Bild Vermählung Marias den Hintergrund bestimmt für eine hiervon unabhängige Form der bildlichen Vordergrundgestaltung. Die Systematisierung des Raums in der Zentralperspektive Albertis prädestinierte eine spezifische, geometrisch reduzierte, überwiegend orthogonale Bauköperkonstruktion. Alle hiervon abweichenden, geometrisch komplexeren Dinge, wie menschliche Körper, mussten, jenseits der Perspektivkonstruktionsregeln, ,frei entwickelt werden: „Im Innern des starren Käfigs der Zentralperspektive ließen sich freizügige, anmutige Körper erschaffen, die ohne jeden Rückgriff auf Geometrie und hinsichtlich ihrer intuitiv befreiten Formen gleichwohl durch sie bedingt entworfen [werden konnten]."7

Diese Zusammenhänge bildräumlicher Funktionalität der neuzeitlichen Perspektivregeln wirken bis heute nach - zum Beispiel in der geometrisch einfachen, strengen und reduzierten Gestaltung der Fassaden des Potsdamer Platzes in Berlin, wie sie insbesondere im Kontext von hier produzierten Werbekampagnen immer wieder sichtbar wird. Das seit den I990er Jahren im Zentrum Berlins gebaute Areal ist vor allem seit seiner Fertigstellung in den frühen 2oooer Jahren überproportional häufig als Location zur Umsetzung von Werbekampagnen verwendet worden. Die wohl geordneten und weitgehend werbebefreiten Fassaden des 
Ensembles bieten für Schauspieler, Moderatoren, Modelle oder Produkte einen besonders ergiebigen, weil kontrollierbaren, neutralen und universell einsetzbaren Bildhintergrund. Die Systematisierung der Raumbildung am Potsdamer Platz scheint auf ähnliche Gesetzmäßigkeiten zurückführbar zu sein, wie sie im Blick durch die , finestra aperta ' der Zentralperspektive Leon Battista Albertis festgelegt wurden und seither den Status allgemeingültiger Richtigkeit erlangt haben.

Bereits in der Renaissance wurde versucht, die Beschränkungen der Perspektivkonstruktion auf die regelrechte und systematische Repräsentation einfacher geometrischer Raumkonstellationen zu überwinden. Ein erster Schritt in diese Richtung stellt laut Robin Evans die von Piero della Francesca entwickelte sogenannte „Andere Methode“, eine bildpunktbezogene Perspektivkonstruktionstechnik dar, mittels derer prinzipiell alle Arten von Gegenständen zeichnerisch erfasst werden konnten, unabhängig davon, welche geometrische Komplexität sie hatten oder in welcher Ausrichtung zur Bildebene sie standen. ${ }^{8}$ Verwandt ist diese Art der Perspektivkonstruktion wiederum mit Methoden der Zeichnungserstellung von Albrecht Dürer, bei der eine direkte Übertragung von ,Werten der Außenwelt“ in Werte bildlicher Repräsentation erfolgte, indem Raumpunkte von Gegenständen mit Hilfe von Apparaten in die Fläche eines Bildes übertragen werden (Abb. 2). Die direkte Verbindung zu einer physisch vorhandenen Realität, im Sinn einer abtastenden, punktuellen Datenerfassung, einer „Auflösung von Oberflächen in Konstellationen von Punkten"9, kann schließlich als Vorläufer aller Verfahren verstanden werden, die den Inhalt eines Bildes indexikalisch aus der Realität beziehen. Die Faszination für die Fotografie speist sich, insbesondere in ihren Anfängen, aus genau dieser, nabelschnurartigen ' ${ }^{\text {Io }}$ Verbindung zur Wirklichkeit.

Neue Kameragesten „Was ist Perspektive? - Wenn eine Leiche ein Auge zukneift."II Der Kunsthistoriker Erwin Panofsky unterscheidet in seinem vielzitierten Aufsatz Die Perspektive als symbolische Form ${ }^{12}$ von 1927 zwischen einer die Systematik des körperlichen Sehens repräsentierenden ,perspectiva naturalis“ und einer dieses Sehen als Modell im perspektivischen Bild systematisierenden ,perspectiva artificiales“. Es geht Panofsky darum herauszustellen, dass die starre Perspektivkonstruktion der Renaissance 


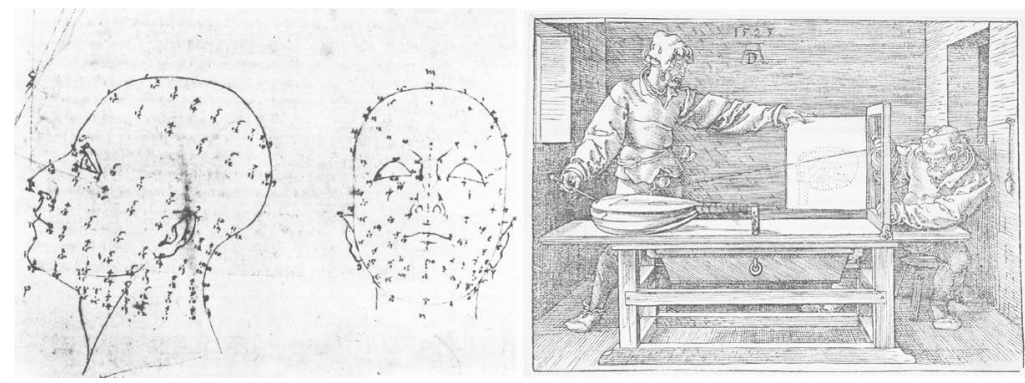

2 Links: Piero della Francesca, Aufsichten und Ansichten der orthogonalen Projektion eines gekippten Kopfes, ca. 1475; rechts: Albrecht Dürers Zeichenmaschine Dürerscheibe, 1525

auf verschiedenen Ebenen in gewaltiger Differenz steht zum menschlichen Sehvorgang. Die Überwindung dieser Differenz zwischen der mathematisch-geometrisch hergeleiteten Bildkonstruktion der Perspektive und dem individuellen, körpergebundenen Seheindruck, ist in der Folge nicht nur ein zentrales Motiv der Weiterentwicklung zeichnerischer und malerischer Ausdrucksstrategien gewesen, sondern sie ist zudem ein treibendes Moment der technischen Genese von Kamerasystemen. Ein Zitat des amerikanischen Kunstkritikers Jonathan Crary aus dem Text Die Modernisierung des Sehens verdeutlicht, mit welchem übermenschlichen, visuellen Absolutheitsanspruch die Fotoaufnahme noch zu Beginn des 20. Jahrhunderts belegt wurde: „Die Öffnung der Kamera korrespondierte mit einem einzigen, mathematisch definierbaren Punkt, von dem aus die Welt logisch deduzierbar und repräsentierbar war. Auf Naturgesetzen, d. h. der geometrischen Optik beruhend, bot die Kamera einen unfehlbaren Aussichtspunkt auf die Welt. Die sinnliche Wahrnehmung, die in jeder Hinsicht vom Körper abhängig war, wurde zugunsten der Repräsentationen dieses mechanischen, monokularen Apparats, deren Authentizität jenseits allen Zweifels verortet wurde, verworfen."13

Diese von Crary über die Entstehungsmodalitäten früher Fotografien vorgetragenen Spezifika einer systematischen, objektivierenden bildlichen Raumkontrolle sind in aktuellen Kamerasystemen derart nicht mehr maßgebend. Die Bildaufnahme mit heute verbreiteten, sogenannten Action-Camcordern zum Beispiel ist weder unfehlbar, noch „starr“ oder ,unbeweglich“"I4. Meistens 
werden diese Kameras nicht einmal mehr über die Kontrolle des wahrnehmenden Auges geführt, sondern sie folgen den Bewegungen der Glieder des Körpers, an die sie gekoppelt werden ohne die Notwendigkeit der Nutzung einer Suchereinheit. Die körperliche Komponente heutiger Fotoaufnahmepraxis von solchen „Actioncams“ kann dabei zurückgeführt werden bis zu optischen Wahrnehmungsapparaturen, wie sie zu Beginn des I9. Jahrhunderts entwickelt wurden. Crary beschreibt die in jener Zeit sich verbreitende Auffassung, Wahrnehmen und apparatives Bildermachen könnten jeweils nicht von den Dispositionen des menschlichen Körpers entkoppelt verstanden werden, als eine „körperliche Dichte des Sehens“. ${ }^{15}$

Bei Smartphonekameras ist zwar die visuelle Kontrolle der Kamera nach wie vor von zentraler Bedeutung, jedoch wird nicht mehr mit Hilfe eines Suchers durch die Optik eines Fotoapparates hindurch eine Szene mit dem Ziel der Aufnahme eines Fotos betrachtet, sondern es wird auf einen mit Abstand zu den Augen gehaltenen Monitor geblickt - zwei völlig unterschiedliche Methoden und Gesten fotografischen Handelns. Der einäugige Blick durch den Sucher einer Kamera verlangt vom Fotografen eine gewisse Abstraktionsfähigkeit, denn das ,gesuchte Bild' ist nur im Bewusstsein der Fotografin als Imagination existent, zum fotografischen Bild wird es erst später, wenn der eigentliche Aufnahmevorgang in der Vergangenheit liegt. Bei monitorgesteuerten Kameras wie Smartphones hingegen tritt das Foto von Beginn an als sichtbares, äußeres Bild in Erscheinung. Das Foto spricht dann nicht länger von einer in die Zukunft verschobenen Vergangenheit, sondern es erscheint als Bild, noch bevor es zu einem Foto gefriert. Es wird zum Teil der Situation, ist selbst, lebendig' wie die beobachtete Szene auf der anderen Seite der Kamera. Wird die traditionelle Funktionalität des fotografischen Bildes von Roland Barthes in seinem Essay Die helle Kammer mit dem Diktum „Es ist so gewesen" ${ }^{16}$ charakterisiert, so nennt die Medientheoretikerin Kathrin Peters den Umgang mit derart momentbezogen aufgenommenen Kamerabildern, die eher einen performativen Akt (die „körperliche Dichte des Sehens“") zeigen, als zu Zwecken der Erinnerung aufgenommen wurden, eine „Prozessierung von Momenthaftigkeit". ${ }^{17}$

Weil das Live-Bild der Kamera heute dem eigenen Handlungsraum zugehört, kann es vor und hinter der Kamera eine krea- 
tive, spielerische, ja performative Jetztbezogenheit evozieren - im Sinn einer Umkehrung der eigentlich distanzierenden Grundtendenz bildlicher Erscheinungen. Wird nicht unreflektiert durch das Monitorbild ,hindurch' auf das sichtbare Sujet geschaut, sondern wird es als aktives, die Entwicklung der Szene beeinflussendes Moment gesehen, dann kann hierdurch eine wechselseitige Dynamisierung zwischen Fotograf, Bild und Szene sich einstellen. Das Monitorbild wirkt dann wie das Scharnier einer performativen Verhandlung zwischen späterem Foto und Szene. Eine derartige Verflechtung zwischen dem Livebild eines Kameramonitors und dem echtzeitlichen Bildmotiv selbst sah der Kunsthistoriker und Medientheoretiker Hans Belting erstmals bei displaygesteuerten Videocamcordern der I990er Jahre Wirkung entfalten. ${ }^{18}$ Die digitale Bildsteuerung von mit dem Internet verbundenen Smartphonekameras hat eine entsprechend nachhaltige Veränderung der Funktionalität des fotografischen Bildes zur Folge, die sich besonders anschaulich zeigen lässt, indem Fotografien gegenübergestellt werden, die auf verschiedenen Fotoplattformen des Internets zu einem Suchbegriff gelistet werden. Der Vergleich von Bildern zu „Potsdamer Platz“ zum Beispiel bringt ganz unterschiedliche Arten von Fotos und Methoden ihrer Verbreitung im Netz zum Vorschein (Abb. 3): Die Google-Bildersuche als Meta-Suchmaschine macht Fotos aus einem großen zeitlichen Intervall sichtbar. Hierbei wird das Internet überwiegend als Bild-Datenbank, als Wissensspeicher zugänglich gemacht. Die erste kommerziell erfolgreiche Fotosharingplattform Flickr demgegenüber ist eher wie ein onlinegeschalteter Fotoclub konzipiert, in dem kompetitiv die Qualität von Fotografien diskutiert wird. ${ }^{19}$ Bei Tumblr und vor allem auch auf Facebook spielen die Fotos demgegenüber eine stärker kommunikative Rolle - sie sind interaktives Ausdrucksmittel zur Sichtbarmachung von erlebten Ereignissen. Diese kommunikative Funktion wird bei den visuell ausgerichteten Instant-MessagingDiensten Instagram oder Snapchat dann zur eigentlichen Funktion der Aufnahme: eine unmittelbare fotografische Veräußerlichung eigenen, gegenwärtigen Handelns.

Diese Form von Individualisierung des fotografischen Blicks auf die Welt steht selbstredend am Ende einer längeren Entwicklung. Der Anfang privater, nicht professioneller Fotoproduktion beginnt ab I890, als George Eastman unter dem Namen ,Kodak eine Serie von für die damalige Zeit besonders nutzerfreundlichen, 

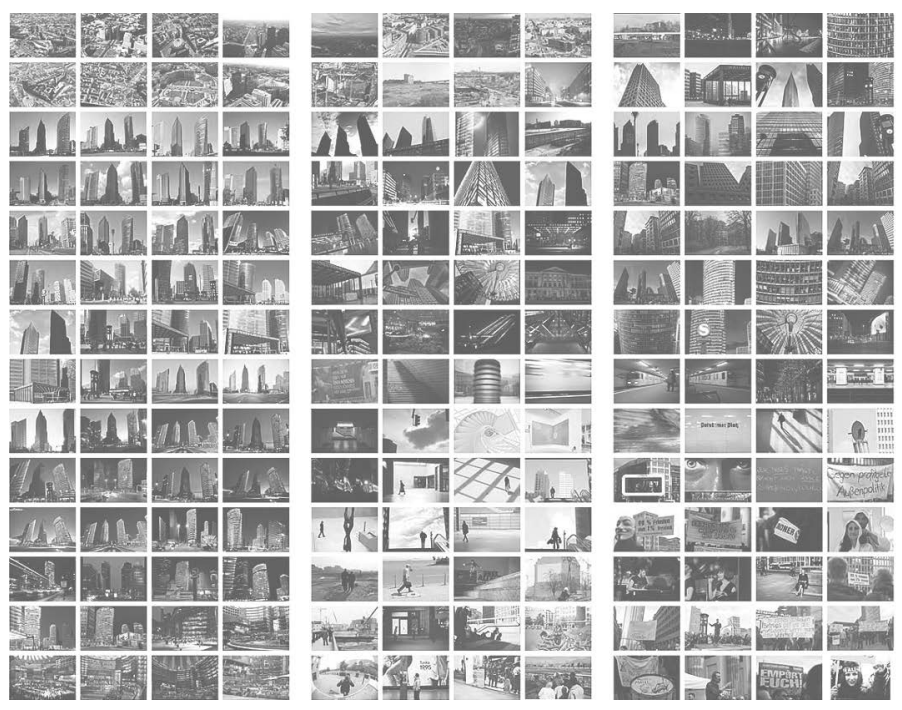

3 Die jeweils 56 ersten Fotos, die bei einer Internetsuche zu dem Begriff ,Potsdamer Platz' auf unterschiedlichen Plattformen erscheinen. Von links nach rechts: Google-Bildersuche, tumblr, flickr, facebook, instagram.

leichten, kompakten und günstigen Handkameras auf den Markt brachte. Vor der Einführung dieser Kamerasysteme wurden Fotografien von Privatleuten überwiegend konsumiert, nicht aber selbst aufgenommen. Im Kontext dieser Individualisierung fotografischer Mittel und eines sich zur selben Zeit entwickelnden Pauschaltourismus festigte sich eine bereits mit der Aufklärung beginnende „Seh-sucht“, ${ }^{20}$ eine Fokussierung der visuellen Aneignung und Bewertung von Umwelt. Der Definition des Historikers und Tourismusforschers Cord Pagenstecher nach führte diese neue Art des Sehens schließlich dazu, dass aus dem Standort (im Englischen site) dessen Anblick (im Englischen sight) wurde. Die „Sehenswürdigkeit“ und deren ,Besichtigung“ verbanden sich zum ,,Sightseeing“, der ,touristische Blick“" wurde geboren. ${ }^{21}$ Die parallel sich entwickelnden Produktions- und Vermarktungsstrategien der Fotound Tourismusindustrie verstärkten die Koppelung der Freizeitaktivitäten des Reisens und Fotografierens. Beide Branchen etablierten allgemeingültige Standards einer visuellen Repräsentation touristischer Ziele, was zur Folge hatte, dass die Frage, was und 


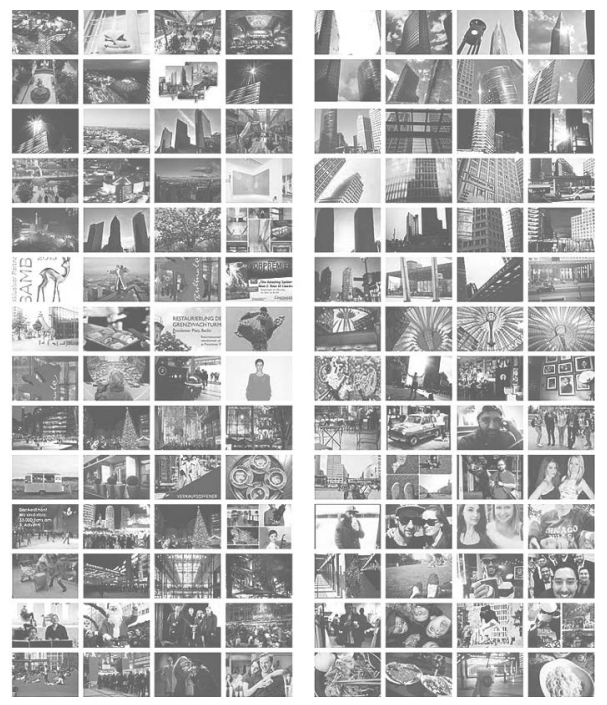

wie zu fotografieren war einer immer stärker werdenden Normierung folgte. Der Medienphilosoph Vilém Flusser stellt die damit einhergehende unflektierte, ja bewusstlose Art, mit der Touristen sich die Welt seither fotografisch erschließen besonders heraus: „Der Knipser kann die Welt dann nur noch durch den Apparat und in den Fotokategorien ansehen. Er steht nicht ,über' dem Fotografieren, sondern ist von der Gier seines Apparates verschlungen, zum verlängerten Selbstauslöser seines Apparates geworden. Sein Verhalten ist automatisches Kamera-Funktionieren. Ein ständiger Fluß bewußtlos erzeugter Bilder ist die Folge." ${ }^{22}$

Was Flusser bemängelt ist anders ausgedrückt die fehlende Reflexion, ja die Missachtung der medialen Prägung des fotografischen Aufnahmevorgangs. Allein der aus einer reflektierten Aufmerksamkeit heraus agierende Fotoprofi kann die notwendige Abstraktion auf die besondere Geste des fotografischen Aufnahmeaktes, auf die besondere „Geste des Fotografierens“23 vollziehen, um so den Blick auf die Medialität des Verfahrens zu wenden. Nur in einem reflektierten, medial bewussten Zustand ist es möglich mit der Kamera neue, andere, ungesehene Aspekte eines Ortes, einer Szenerie oder eines Ereignisses fotografisch sichtbar zu machen. Dass auch Architektur in diesem Kontext eine zentrale Rolle spielt, kann beispielhaft an dem House Fallingwater von Frank Lloyd 


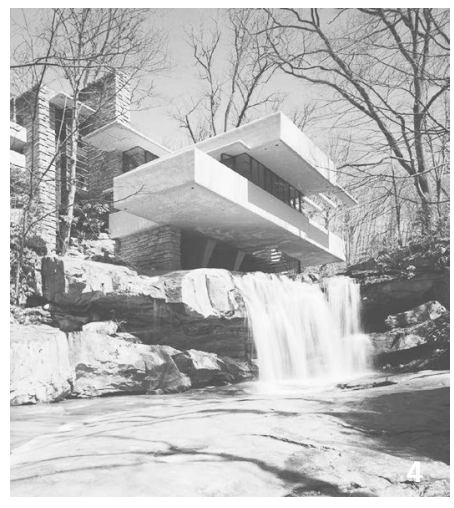

4 Ezra Stoller, Fallingwater, Frank Lloyd Wright, 1971

5 Google-Bildsuche 'House Fallingwater'

Wright gezeigt werden. Die klassische Repräsentation von Architektur durch fotografische Verfahren macht deutlich, wie ein Foto als offenes Fenster in eine scheinbar wirklich anwesende Realität missdeutet werden kann. In kommerziellen, meistens im Auftrag eines Architekten oder einer Bauherrin erstellten Fotografien eines Gebäudes wird häufig versucht dessen Wesen über wenige Aufnahmen möglichst treffend zu bestimmen. Das jedem Architekturinteressierten geläufige Bild des House Fallingwater, insbesondere Ezra Stollers ikonographische Fotographie von I97I, aufgenommen von einem abgesengten Standpunkt, den namensgebenden Wasserfall im Vordergrund (Abb. 4), zeigt entsprechend nicht nur eine mögliche Ansicht des Gebäudes, sondern es hat selbst den Status eines allgemeingültigen Stellvertreters des Gebäudes eingenommen. Gibt man heute auf den bekannten Fotoplattformen des Internets (etwa bei der Google-Bildersuche) die Begriffe ,House Fallingwater' in die Suchmasken ein, dann erscheint eine unüberschaubare Anzahl von Bildern, in denen genau dieses ikonografische Foto des Ortes immer wieder reproduziert wird (Abb. 5). Die Wirkungsrichtung fotografischer Bedingung kann sich dabei schließlich auch umkehren. Nicht die Fotografie ist dann die Erscheinung zweiter Ordnung, ist die Ableitung oder der Stellvertreter von etwas in Wirklichkeit bestehendem, sondern die Wirklichkeit hat sich zu messen an der fotografischen Erscheinung, an der Wahrheit eines ikonografischen, immer wieder reproduzierten Bildes. Die touristischen Besucher und Besucherinnen eines Ortes, deren Erwartung derart fotografisch vorgeprägt ist, können dann häufig dem Umstand ihrer eigenen Anwesenheit an diesem ,Foto- 


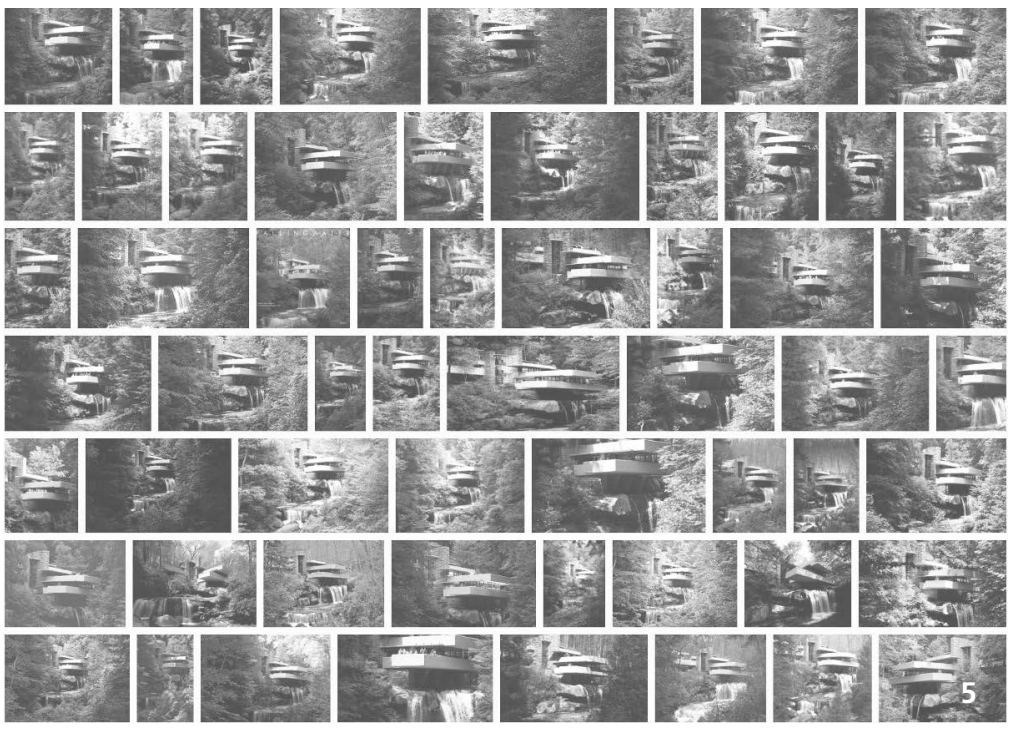

Ort' nur noch dadurch Ausdruck verleihen, dass sie eben dieses Foto selbst nochmals reproduzieren.

Die in der klassischen fotografischen Repräsentation von Architektur nachwirkende perspektivische Raumsystematisierung lässt das Bild als Durchsicht durch ein offenes Fenster in seiner physischen Anwesenheit regelrecht verschwinden. ${ }^{24}$ Denn Bilder zeigen etwas, dass sie selbst nicht sind - sie führen etwas Abwesendes als anwesend vor. Den imaginären, physisch nicht anwesenden Teil eines Bildes, der dessen ,Durchschauung“ erst möglich macht, bezeichnet der Phänomenologe Edmund Husserl als das Bildobjekt. ${ }^{25}$ Das Besondere am Bildobjekt ist, dass es über keine physikalischen Eigenschaften verfügt, dass es materiell nicht greifbar ist, dass es nur sichtbar ist. Der anwesende, berührbare Bestandteil der bildlichen Erscheinung ist der Bildträger auf beziehungsweise in dessen Oberfläche das Bildobjekt vorstellig wird. In der klassischen Bildwirkung bleibt dieser Bildträger meist unbeachtet - er wird, ähnlich dem Glas beim Blick durch ein Fenster, unsichtbar. Bildbetrachtung ist dem Philosophen und Bildtheoretiker Lambert Wiesing nach daher genau genommen die „Betrachtung eines Nichts“. ${ }^{26}$ Eine Störung oder Abbremsung dieser widerstandslosen Durchschauung findet immer dann statt, wenn die 
(geometrische) Bedeutung des Bildobjekts nicht eindeutig ist, wenn ein Widerstreit entsteht zwischen der anwesenden Bildfläche, dem Bildobjekt und dem bedeutenden Sujet. Eine Reflexion dieses dreigliedrigen Widerstreits bildlicher Wirkung ist gerade heute von zentraler Bedeutung, da uns immer mehr Bilder umgeben, die aufgrund ihrer Erscheinungsform ,extrem durchsichtig' geworden sind. Die hochaufgelösten Displays moderner Smartphones und Tablets, die bei der Firma Apple in Anlehnung an die Qualität des natürlichen Sehens als ,Retina-Displays ' bezeichnet werden, lassen heute die Artefakte einer bis vor kurzem noch sichtbaren digitalen Bilderzeugung immer mehr in den Hintergrund rücken. Sie suggerieren eine ,natürliche Bilderscheinung', als würde homogenes und kontinuierliches Licht in unsere Augen emittiert werden. Doch gerade derart natürlich und untechnisch erscheinende Monitorbilder sind ihrem Wesen nach die abstraktesten Arten von Bildern überhaupt. Vilém Flusser warnte in diesem Sinne bereits Anfang der I980er Jahre: „Bilder sind Vermittlungen zwischen der Welt und dem Menschen. Der Mensch ,ek-sistiert', das heißt, die Welt ist ihm unmittelbar nicht zugänglich, so daß Bilder sie ihm vorstellbar machen sollen. Doch sobald sie dies tun stellen sie sich zwischen die Welt und den Menschen. Sie sollen Landkarten sein und werden zu Wandschirmen: Statt die Welt vorzustellen, verstellen sie sie, bis der Mensch schließlich in Funktion der von ihm geschaffenen Bilder zu leben beginnt. Er hört auf, die Bilder zu entziffern, und projiziert sie statt dessen unentziffert in die Welt ,dort draussen“ $[\ldots] . " 27$

Fotografische Raumkonstruktionen Wird der Blick durch die Bildoberfläche mehrerer Fotografien hindurch auf das , hinter' ihnen sich mitunter befindende, gemeinsame Bildsujet gerichtet, dann kann in einer Art Umkehrung des Fotoaufnahmeprozesses durch die Rückberechnung von Geometrie auf die dreidimensionale Verfasstheit des abgelichteten Ortes geschlossen werden. Die Haupttriebfeder für die Erforschung derartiger Techniken liegt im Interesse der großen Internet-Kartendienste begründet, eine möglichst lückenlose, dreidimensionale, fotorealistische Repräsentanz der Welt online zugänglich zu machen, ohne mit großem technischen wie finanziellen Aufwand eigenständig foto- und laserbasiert den öffentlichen Raum scannen zu müssen (wie Google es zum Beispiel für seinen Fotokartendienst Streetview tut). Modeling the World 
from Internet Photo Collections heißt der Titel einer Forschungsarbeit von Noah Snavely, Steven Seitz und Richard Szeliski, in der ein solcher Ansatz entwickelt und beschrieben wird. ${ }^{28}$ Eine zentrale Stufe dieses Verfahrens wird dabei als „Structure from Motion“ bezeichnet. Hierbei werden in einer Art von simulierter Kamerabewegung über Einzelbilder hinweg Raumdaten aus gemeinsamen Elementen von Fotos abgeleitet, die dasselbe Objekt aus unterschiedlichen Perspektiven zeigen. Das spezielle Problem bei dieser Methode ist, dass zunächst weder die Positionen der aufnehmenden Kameras bekannt sind, noch die räumliche Lage der Bildobjekte untereinander. Auch liegen die geometrisch-optischen Kennzahlen der verwendeten Kameras zunächst weder vor, noch sind sie einheitlich bestimmt (so wie es bei den Messbildkameras photogrammetrischer Verfahren zentrale Voraussetzung ist). Dennoch ist es mit Hilfe eines rechenintensiven Prozesses möglich mittels Mustererkennungsverfahren auch in sehr großen Fotogruppen jene Aufnahmen zu detektieren, auf denen gleiche Elemente eines Ortes abgelichtet sind, unabhängig davon, wie ihre zweidimensionale, projektive Ableitung im Foto in Erscheinung tritt. Am Ende des algorithmischen Verfahrens steht die Überführung von in mehreren Fotos sichtbaren, gleiche Ortselemente zeigenden Bildpunkten in eindeutig bestimmte Koordinaten eines virtuellen Digitalmodells.

Die eigentliche Rekonstruktion der Raumdaten stellt hierbei mitunter nicht den interessantesten Aspekt der Methode dar. Vielmehr sind die hierbei zusätzlich sichtbar werdenden Verortungen der ehemaligen Fotografinnen und Fotografen äußerst bemerkenswert (Abb. 6). Es wird nicht nur der , kollektive Bildraum` abgelichteter Oberflächen visualisiert, sondern auch die allen Fotos zugrundeliegenden Aufnahmestandpunkte und Blickrichtungen werden als komprimierter Zeitraum des fotografischen Handelns sichtbar. In den auffälligen Lücken der so berechneten 3D-Modelle zeigen diese Visualisierungen auf eindrückliche Weise, bis wohin das visuelle Interesse der hier in einem Zeitfenster fotografiert habenden Besucher jeweils gegangen ist (Abb. 7). Schaut man zudem die verwendeten Einzelfotografien genauer an, so fällt auf, dass auch große Teile der Bildinhalte in den dreidimensionalen Rekonstruktionen nicht mehr in Erscheinung treten. Alles dynamische, alles was die Differenz der persönlichen Betrachtung des Ortes im Medium Foto besonders kennzeichnet wird nicht verarbeitet. Der Bildbearbeitungsprozess selbst ist hierfür maßgeblich verantwortlich, denn 


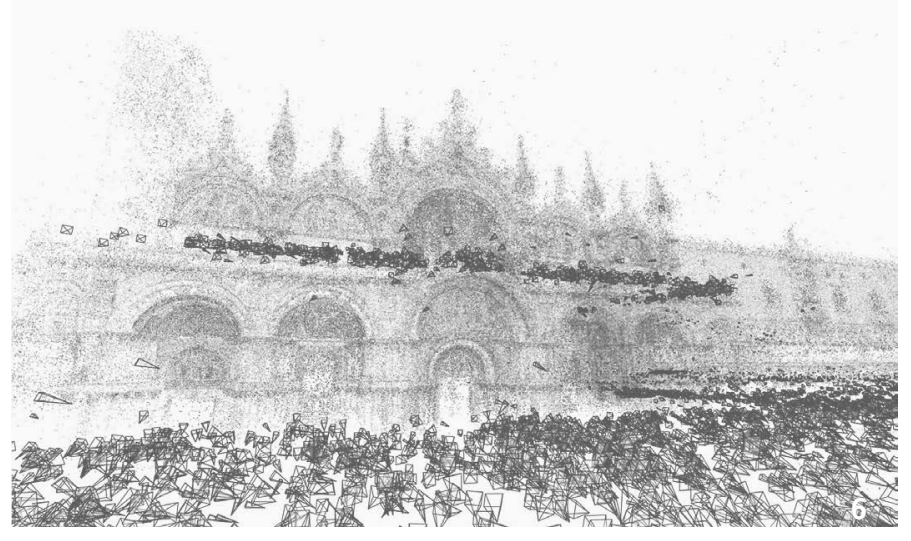

sämtliche Stufen der Rekonstruktion sind darauf ausgerichtet nur wiederholt sichtbare Bildobjekte zu prozessieren. Diese ,Querschnittsberechnung ' lässt einen ,Mittelwert-Raum' entstehen, in dem die individuellen Differenzen der fotografischen Anschauung eliminiert werden. Es stellt sich eine im wahrsten Sinne des Wortes , oberflächliche Evidenz' zwischen Ort und Rekonstruktion ein, die von der Möglichkeit einer Gleichsetzung zwischen fotografischer Bildlichkeit und baulicher Realität ausgeht. Die Fotografie jedoch repräsentiert gerade mehr als nur stillgestellte Artefakte. Der ,fotografische Überschuss“, der in diesen Rekonstruktionen verloren geht, stellt ein zentrales Element fotografischer Bedeutungsbildung dar: „Es ist ihre zeitliche Dimension, in der sich das ganze Paradox der Photographie in ihrer gebräuchlichsten Version offenbart. Als plötzlicher Schnitt in die sichtbare Welt ist die Photographie das Mittel, die solide und kompakte Wirklichkeit der alltäglichen Wahrnehmung in eine unendliche Vielfalt flüchtiger Ansichten aufzulösen [...].“"29

Die in den Fotos hinterlegte individuelle Zeitlichkeit wird mit den algorithmischen Verfahren einer reinen Summierung monokularer Sichtweisen nicht zugänglich gemacht. Ziel des Prozesses ist es gerade nicht, widersprechende Aussagen über eine Szene in Ermöglichung von Differenz integrativ zu verbinden, sondern die heterogenen, bildlichen Zugänge auf eine singuläre Wahrheit herunter zu brechen. Die scheinbare Evidenz, die sich hierbei einstellt zeigt sich in Form eines horizontlosen, unverschatteten Allgemeinplatzes. Das verlorene Potenzial der inneren Evidenz 


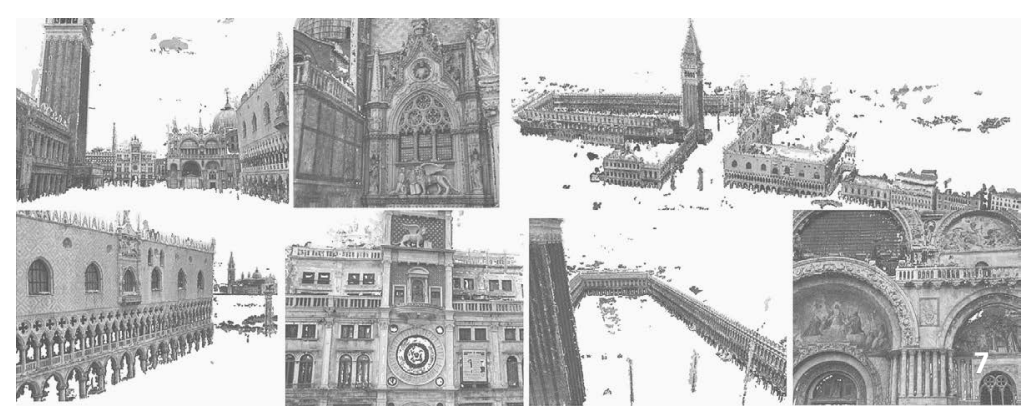

6 3D-Simulation des Markusplatzes in Venedig bestehend aus 4.515.157 Punkten, berechnet aus 14.079 Fotos der Internetplattform Flickr. Die schwarzen Pyramiden zeigen die rückberechneten Aufnahmestandpunkte und Blickrichtungen der Fotografen.

7 Serie berechneter Ansichten dichter Punktwolken des Markusplatzes in Venedig

jedes einzelnen Bildes, ihre ,ikonischen Differenzen“30 werden nur in den Lücken und Brüchen der entstehenden 3 D-Modelle sichtbar: Hier verbergen sich die Ausschnitte der Fotografien, deren Evidenz eine andere war, als die mehrerer , benachbarter' Aufnahmen.

Entwurf foto-topologischer Raumrelationen Trotz dieser, der Verfahrenslogik des Prozesses geschuldeten Auslöschung zentraler Potenziale des fotografischen Bildes, ergibt sich, jenseits der eigentlichen Rekonstruktion von Raumkoordinaten - auf dem algorithmischen Weg dorthin - in einer Vorstufe der eigentlichen räumlichen Rekonstruktion mittels Structure from Motion, eine sehr interessante Form fotobasierter Beschreibung von Orten. Es handelt sich dabei um Abhängigkeitsgrafen, in denen die wiederholte Sichtbarkeit gleicher Elemente zwischen verschiedenen Fotos erkennbar wird (Abb. 8). Ohne das zu diesem Zeitpunkt des Prozesses die räumlichen Zusammenhänge der Szenerie bereits bekannt wären, ergeben sich in Visualisierungen dieser Fotoreferenzen Hinweise auf die Funktionalität einer Szene in ihrer multiplen Wahrnehmung mittels Kameras. Die Repräsentation eines Ortes durch derartige Abhängigkeitsgrafen zeigt weder reine Baulichkeit noch reine diagrammatische Verbildlichung von Bewegungsmustern. Es 

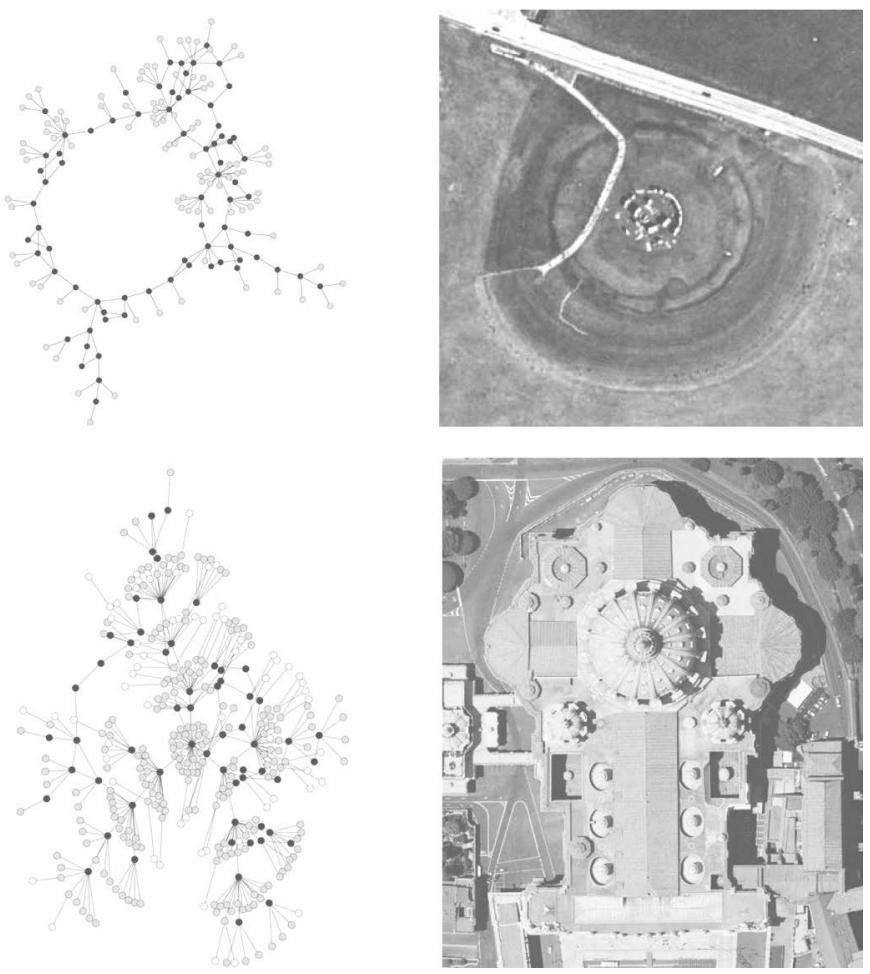

8 Darstellungen von Fotosammlungen (Stonehenge, Petersdom) als ,Foto-Konnektivitäts-Graphen' (sog. ,Skeletal Graphs') und Luftbild

werden hier vielmehr die normalerweise nicht synchron darstellbaren außerbildlichen Augenpunkte der Fotografinnen und Fotografen und die im Bild sichtbaren, geometrischen Wirklichkeiten des Ortes miteinander kurzgeschlossen. In solchen relationalen Systemen sind die absoluten geometrischen beziehungsweise geografischen Standpunkte von Subjekten und Objekten und deren zeitliche Absolutheiten unbedeutend gegenüber den vektoriellen Bestimmungen ihrer bildlichen wie zeitlichen Kongruenzen. Die im singulären Perspektivbild bestehende räumliche wie zeitliche Stillstellung der Relationen zwischen Subjekt und Objekt, kann beim Vorhandensein einer großen Anzahl von Einzelperspektiven überwunden werden, weil zwischen vielfachem ,Hier' und vielfachem ,Dort' netzartige Zusammenhänge beschreibbar sind. 
Im Kontext stadträumlicher Fragestellungen unterscheidet der Medientheoretiker Stephan Günzel in genau diesem Sinn eine auf geografische Absolutheiten ausgerichtete topografische von einer auf die Bestimmung von Relationen ausgerichteten topologischen Umweltbetrachtung. Die topologische Sichtweise zeichnet sich dadurch aus, „dass von Materialität gerade abstrahiert wird“"31 Sollen die heutzutage im Internet zirkulierenden Fotografien zu einem methodischen Moment architektonischen Entwerfens operationalisiert werden, dann geraten die topologischen Relationen die zwischen den verschiedenen Ebenen einer Gruppe von Fotos entdeckt beziehungsweise hergestellt werden können, also die Logiken und Paradigmen dieser Ordnungssysteme, ins Zentrum der Bedeutungsbildung. Die starre Gerichtetheit der Perspektive, die bereits in den heutigen performativeren Gesten der Fotoaufnahme unterwandert wird, kann in topologisch referenzierten Bildclustern endgültig in den Hintergrund geraten, zugunsten richtungsoffen betrachtbarer Subjekt-Objekt Konstellationen.

Mit dem Bildgraph einer Fotosammlung, die den TreviBrunnen in Rom zeigt ${ }^{32}$, können entsprechend gleichzeitig Äquivalenzen wie auch Abweichungen zwischen der topologisch-diagrammatischen Beschreibung eines Ortes und seiner topografischen Disposition aufgezeigt werden. Das Diagramm dieses Ortes (Abb. 9) untergliedert sich in zwei auffällige Cluster, die nur durch wenige Referenzierungslinien verbunden sind. Die sichtbaren Bildcluster beschreiben hierbei keine räumlichen Zusammenhänge, sondern sie referieren auf zeitliche Äquivalenzen - helle Tagaufnahmen oben und dunkle Nachtaufnahmen unten. Die horizontale Ausrichtung der beiden Verdichtungen dagegen gibt weitgehend die tatsächliche geografische Ausdehnung des Areals wieder. Zwischen topologisch bestimmter Diagrammfigur und geografischer Lage des Ortes kann es aber auch zu Umkehrungen kommen: Das geographische Innen der Struktur kann topologisch ein Außen sein und umgekehrt. So befinden sich am Rand der Verdichtungen des „Konnektivitäts-Graphen“ überwiegend Fotografien, auf denen der Ort nicht wie zu erwarten wäre aus weiterer Entfernung von außen aufgenommen wurde, sondern umgekehrt solche, auf denen überwiegend Nahaufnahmen von Details des Brunnens im Zentrum der Szene zu sehen sind. Räumlich beschrieben geraten Aufnahmen, die den geografisch zentralen Bereich des Ortes abbilden also an den Rand des Diagramms. ,Das Außen“ 


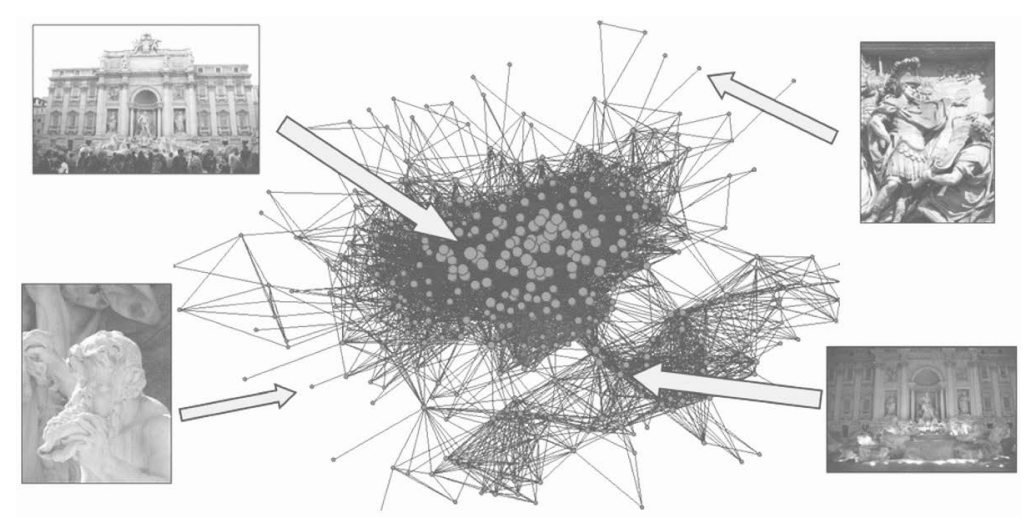

9 Foto-Konnektivitäts-Graph einer Bildersammlung des TreviBrunnens in Rom. Jeder Punkt steht für ein Foto, die Größe der Punkte zeigt die Anzahl gleicher Merkmalspunkte mit anderen Fotos, die Linien verbinden Fotos mit gleichen Merkmalspunkten.

entspricht hier keinem geografischen Außen, sondern transportiert eine qualitative wie quantitative mediale Randständigkeit. Diese Verschiebungen entstehen, weil nicht die Objekte des Ortes selbst topologisch betrachtet werden, sondern virtuelle Beziehungen von Wahrnehmungsakten der am Ort fotografierenden Personen. Die multiple, fotografische Repräsentation des Ortes ermöglicht es, diesen als topologisches Beziehungsfeld zu beschreiben. Die im Kamerabild festgehaltene ,Verkreuzung“ zwischen einem wahrnehmenden Subjekt und den hierbei fotografisch zur Anschauung gebrachten Dingen bleibt jeder Fotografie, zumindest latent eingeschrieben. „Wir erinnern den Blick, der seinerseits in einem Photo erinnert ist. In diesem Sinne ist die Photographie ein Medium zwischen zwei Blicken“.33

Im Sinn einer inwendigen Referenzierung kann der fotografische „Blicktausch“ auch zwischen mehreren Aufnahmen konstruiert beziehungsweise initiiert werden. Die heterogenen Erstellungszeitpunkte einer Sammlung von Fotografien, die Zeiten, auf die das jeweils Abgebildete verweist und die Momente ihrer Rezeption verschränken sich mit dem Raum und den Körpern der Aufnahmen $\mathrm{zu}$ einem integrativen Ganzen. Als mediale Akteure bilden die Fotografien die Scharniere zwischen innen und außen, zwischen 
sehen und gesehen werden, zwischen Subjekt und Objekt. Bauliche Aspekte eines Ortes sind so nicht länger isolierbare, äußere Geometrien, sondern sie sind nicht trennbarer Teil einer Erscheinung, die ohne die sie zur Erscheinung bringenden fotografierenden Subjekte, nicht verfügbar wären. Erinnern tun diese Kreuzungen und Verschachtelungen dessen, was uns in der Erfahrung zwischen Subjekt und Objekt begegnet an die Phänomenologie der Wahrnehmung von Maurice Merleau-Ponty. Er schreibt: „Sehender-Sichtbares $=$ Projektion-Introjektion. Eines wie das andere aus einem einzigen Stoff gewonnen." ${ }^{34}$

Szenenentwurf statt Szenenrekonstruktion Unter dem Titel Mit anderen Augen sehen untersucht die Studentin Hannah Linck in dem Lehr- und Forschungsprojekt „PhotoStitching Reality“ am Institute of Media and Design der TU Braunschweig die topologischen und topografischen Zusammenhängen in einer über das Internet zusammengetragenen Sammlung von Fotos des Münchener Olympiaparks. Hierfür werden die Bilder zunächst in unterschiedlichen Kategorien geordnet. Auf einem Lageplan wird anschließend der Aufnahmestandpunkt sowie die Aufnahmerichtung jedes Fotos mit einem Winkel markiert. Die Kanten dieser Eintragungen zeigen die jeweiligen Bildausschnitte der Fotografien. Von verschiedenen Bildern beginnend wird nun der Rand des Bildausschnitts im Lageplan jeweils so weit verlängert, bis ein nächster (virtueller) Aufnahmestandpunkt eines anderen Fotos getroffen wird. Die entstehenden Blickketten verbinden unterschiedliche Aufnahmestandpunkte und differente Blickrichtungen miteinander. Die Topografie des Geländes wird hierbei zunächst außer Acht gelassen. Dadurch entstehen auch Verbindungslinien zwischen Fotoaufnahmestandpunkten, denen mitunter keine realen Sichtlinien entsprechen können. Die überwiegende Ausrichtung der Kameras ins Zentrum des Areals führt dazu, dass die Referenzierungen früher oder später spiralförmig in die Mitte des Geländes, meist in das Olympiastadion hineinleiten (Abb. IO).

Die den Verkettungen von Aufnahmestandpunkten zugehörigen Fotografien werden anschließend ähnlich einer panoramischen Abwicklung aneinandergefügt. Die Fotoketten zeigen dabei jedoch keine innerbildlichen Kontinuitäten, da sie als Blickreihen nicht nur unterschiedliche Aufnahmestandpunkte, sondern auch differente Blickrichtungen miteinander koppeln. Um die für 


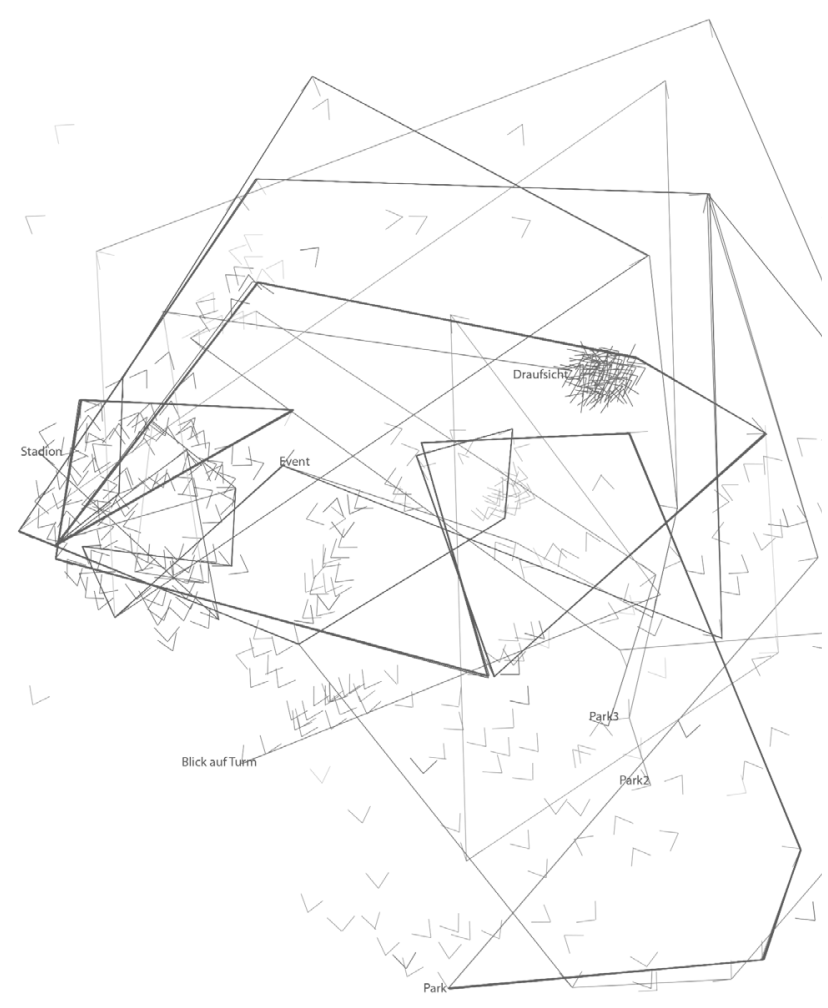

10

den Ort wichtige topografische Prägung in der Bearbeitung nicht gänzlich zu unterschlagen, werden die in Reihe gefügten Bilder vertikal derart zueinander verschoben, dass die Horizonte benachbarter Bilder auf gleicher Ebene liegen (Abb. I I). Die Höhen der unteren Fotokante und ihre Abstände zu den Horizontlinien können dadurch abgetragen werden. Die entstehenden Höhenprofile werden abschließend in der Form ihres ursprünglichen Verlaufs materialisiert. Die Zwischenräume benachbarter Kanten werden mit Flächen überspannt. Die hierbei entstehende Struktur (Abb. I2) repräsentiert Aspekte der, visuellen Benutzung' des Ortes, seiner gesamträumlichen Topografie und seiner Einzelbauten integrativ. Die Topografie des Modells zeigt die Höhenzusammenhänge des Geländes nur medial transformiert entsprechend der Kamerahaltung vieler fotografierender Besucher: Der Hochpunkt des Ortes, der Fernsehturm, wird zum tiefsten Punkt, weil der Horizont der 


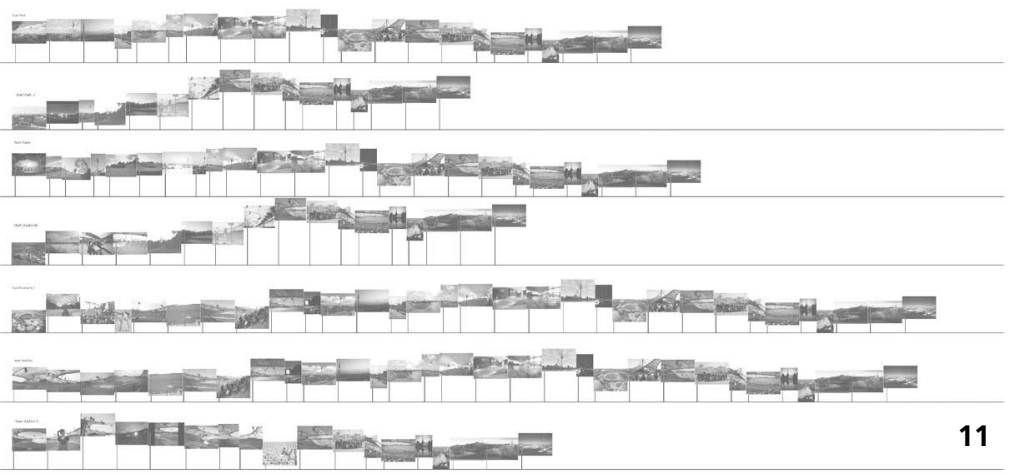

Hannah Linck, Mit anderen Augen sehen, fotografischmediale Analyse des Münchener Olympiaparks

10 Fotoaufnahmestandpunkte mit Blickketten (Ausschnitt)

11 Panoramische Blickketten

von hier nach unten aufgenommenen Fotos, an ihren oberen Rand sich verschiebt und die zugehörigen Abwicklungsstreifen nach der angewendeten Bildordnungsregel damit besonders schmal werden beziehungsweise weit nach unten wandern. Die auffällige strukturelle Verdichtung im Modell wiederum geht auf die engen und dichten Blickbeziehungen innerhalb des Olympiastadions zurück. Dieser Bereich wird gleichzeitig zum Hochpunkt der Modellstruktur, weil (nachdem das Stadion nur noch selten für Veranstaltungen genutzt wird) viele Besucher ihre Kameras nach oben in die charakteristische Dachkonstruktion des Entwurfs von Behnisch \& Partner mit Frei Otto richten.

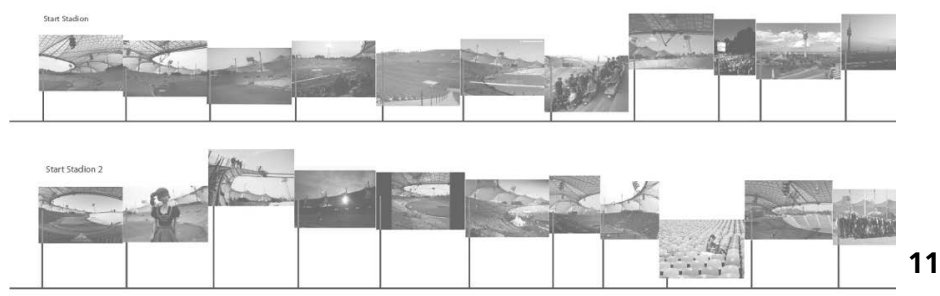




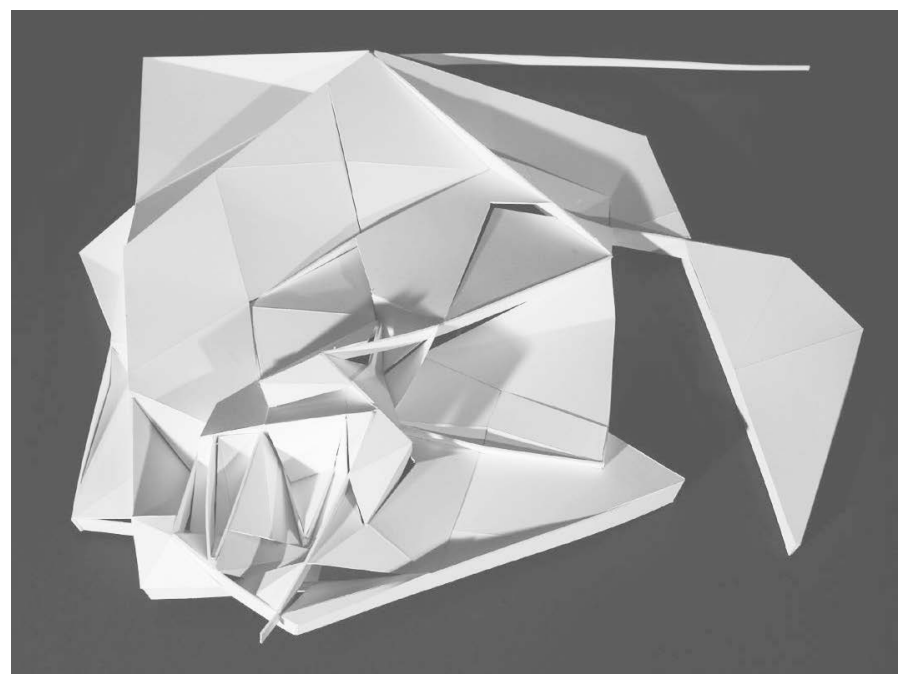

12 Hannah Linck, Mit anderen Augen sehen, fotografischmediale Analyse des Münchener Olympiaparks, Modellfoto

Die Materialisierung der topologischen Aufnahmeanalyse zeigt eine Räumlichkeit, in der die Medialität der Ortswahrnehmung nicht nur latent und verborgen enthalten ist, sondern in der diese Medialität selbst sichtbar zur Geltung gebracht ist. Die Vielheit der perspektivischen Aufzeichnungen wird nicht auf einen durchschnittlichen ,Mittelwert-Raum' heruntergebrochen, sondern mittels ihrer wird eine mögliche ,Zuständlichkeit“ der individuellen, fotografischen Aneignung des Ortes realisiert. Hiermit wird deutlich, wie das in jeder Fotografie in Abschattung befindliche phänomenologische ,plus ultra' eines mannigfaltigen räumlichen Potenzials sich als architektonisches Entwurfsmittel entfalten lässt. Mit Hilfe einer auf die Wirklichkeit, also den baulichen ,Bestand' und seine Benutzer sich beziehenden fotografisch-medialen Analyse, kann eine diese Analyse entwerferisch überschreitende Transformation ausgelöst werden, die wiederum den Ausgangspunkt für eine architektonische Konkretisierung darstellt. ${ }^{35}$ 


\section{Anmerkungen}

1 Vgl. z.B. Herta Wolf (Hg.), Paradigma Fotografie. Fotokritik am Ende des fotografischen Zeitalters, Frankfurt a. M. 2002.

2 Vgl. Kathrin Peters, Sofort-Bilder. Aufzeichnung, Distribution und Konsumtion von Wirklichem unter dem Vorzeichen der Digitalfotografie, unter: www.medienkunstnetz.de/themen/foto_byte/sofort_ bilder/scroll/ [03.2015].

3 Vgl. Susan Sontag, „In Platos Höhle”, in: Dies., Über Fotografie (New York, 1977), Frankfurt a. M. 2013, S. 29.

4 Vgl. Marshall McLuhan, Die magischen Kanäle. Understanding Media, Frankfurt a. M., Hamburg 1970, S. 17 ff.

5 Vgl. Matthias Eberle und Adrian von Buttlar, "Landschaften und Landschaftsgarten", Deutsches Institut für Fernstudien an der Universität Tübingen: Funkkolleg Kunst, Studienbrief 7, Tübingen 1985, S. 11-50.

6 Robin Evans, „The Projective Cast", Arch+ 137, 1997, S. 41.

7 Ebd.

8 Vgl. ebd., S. $46 f f$.

9 Ebd., S. 48.

10 Vgl. Roland Barthes, Die helle Kammer, Frankfurt a. M. 1985, S. 91.

11 Bruno Taut, Frühlicht 1920-1922. Eine Folge für die Verwirklichung des neuen Baugedankens, Frankfurt a. M. und Wien 1963, S. 16.

12 Vgl. Erwin Panofsky, "Die Perspektive als symbolische Form", in: Ders., Aufsätze zu Grundfragen der Kunstwissenschaft, Berlin 1985.

13 Jonathan Crary, "Die Modernisierung des Sehens", in: Herta Wolf (Hg.), Paradigma Fotografie, Frankfurt a. M. 2002, S.70.

14 Ebd., S. 80.

15 Ebd.

16 Vgl. Barthes, Die helle Kammer (Anm. 10), S.105.

17 Vgl. Kathrin Peters, Sofort-Bilder (Anm. 2).

18 Vgl. Hans Belting, Bild-Anthropologie, München 2001, S. $82 \mathrm{f}$.

19 Vgl. Eve Forrest, „Reflections on Flickr. Everyday photography practices online and offline", in: Reconsidering Amateur Photography, Online-Projekt EITHER / AND, unter: www.eitherand.org/ [12.2013].

20 Vgl. Stephan Oettermann, Das Panorama. Die Geschichte eines Massenmediums, Frankfurt a. M. 1980. 
21 Cord Pagenstecher, "Reisekataloge und Urlaubsalben. Zur Visual History des touristischen Blicks", in: Gerhard Paul (Hg.), Visual History. Ein Studienbuch, Göttingen 2006, S. 169-187.

22 Vilém Flusser, Für eine Philosophie der Fotografie, Berlin 2011, S. 53.

23 Ebd.

24 Vgl. Gottfried Boehm, Studien zur Perspektivität. Philosophie und Kunst in der Frühen Neuzeit, Heidelberg 1969, S. 31 f.

25 Vgl. Edmund Husserl, „Phantasie, Bildbewußtsein, Erinnerung. Zur Phänomenologie der anschaulichen Vergegenwärtigung", in: Eduard Marbach (Hg.), Texte aus dem Nachlaß (1898-1925), Husserliana, Bd. XXIII, Boston, London 1980. Vgl. Lambert Wiesing, Phänomene im Bild, München 2000, hier 2. Auflage 2007, S. 10.

26 Ebd., S. 12.

27 Vilém Flusser, Für eine Philosophie der Fotografie (Anm. 22), S. 9 f.

28 Keith Noah Snavely, Steven M. Seitz, Richard Szeliski, „Modeling the World from Internet Photo Collections", International Journal of Computer Vision, Nov. 2008, 80, 2, S. 189-210.

29 Pierre Bourdieu, Luc Boltanski, Eine illegitime Kunst. Die sozialen Gebrauchsweisen der Fotografie, Hamburg 2006, S. 87.

30 Vgl. Gottfried Boehm, „Grundbegriffe des Bildes. Ikonische Differenz", Rheinsprung 11 - Zeitschrift für Bildkritik, 2011, S. 173.

31 Stephan Günzel, „Topologie und städtischer Raum”, der architekt 3, 2008, S. 9.

32 Vgl. Keith N. Snavely, Scene Reconstruction and Visualization from Internet Photo Collections, Doctoral thesis, 2008, unter: www. cs.cornell.edu/ snavely/publications/thesis/thesis.pdf University of Washington, S. 34 [17.05.2019].

33 Hans Belting, Bild-Anthropologie (Anm. 18), S. 224.

34 Maurice Merleau-Ponty, Das Sichtbare und das Unsichtbare. Gefolgt von Arbeitsnotizen (1960), München 1986, S. 329.

35 Der Textbeitrag geht inhaltlich zurück auf: Philipp Reinfeld, ImageBased Architecture. Fotografie und Entwerfen, Paderborn 2018. 
Matthias Ballestrem

\section{Experimentelles Entwerfen: Methoden des kontrollierten Kontrollverlusts}

Im Entwurfsprozess ist es oftmals ein Zustand völliger Vertiefung in das Gestalten, der zu neuen und einzigartigen Findungen führt und der Architektur ihre spezifische räumliche Sprache verleiht. In diesem Zustand herrscht eine gestalterische Freiheit. Wir arbeiten dann nicht zielgerichtet auf eine Lösung hin, sondern experimentieren intuitiv und selbstvergessen innerhalb eines Möglichkeitsfelds, das vielfältige Ergebnisse hervorbringen kann. Bedingungen für das Erreichen dieses Zustandes sind in der Regel gezielte inhaltliche und mediale Einschränkungen, welche die komplexen Bindungen einer Entwurfsaufgabe temporär ausblenden, um ein bestimmtes gestalterisches Problem experimentell untersuchen zu können. Diese Bedingungen des experimentellen Entwerfens, sowie die Bedeutung der daraus entstehenden Ergebnisse für das architektonische Gestalten und Wirken werden im Folgenden untersucht. Anhand von Beispielen gestalterischer Experimente soll den Aspekten der medialen Bindungen, der Reduktion, der Unschärfe, Intuition und Autorenschaft nachgegangen werden.

Reduktion, Ungewissheit und Unschärfe Beginnen will ich mit einem Beispiel aus der Kunst. Abbildung I zeigt eine Skulptur des Künstlers Joe Fig aus dem Jahr 2008, die Jackson Pollock bei der Arbeit zeigt. Sie stammt aus einer Reihe von kleinen Dioramen, die Fig von berühmten Künstlern bei der Arbeit und deren Atelierumgebungen retrospektiv angefertigt hat. Die Skulptur Pollock wirkt gleichzeitig so lebensecht und historisch plausibel, dass Fotos davon oftmals verwendet werden, um Pollocks Arbeitsweise zu beschreiben. Sie zeigt den Künstler irgendwo im Niemandsland auf einem Podest. Mit seinen umlaufenden Stufen wirkt es 


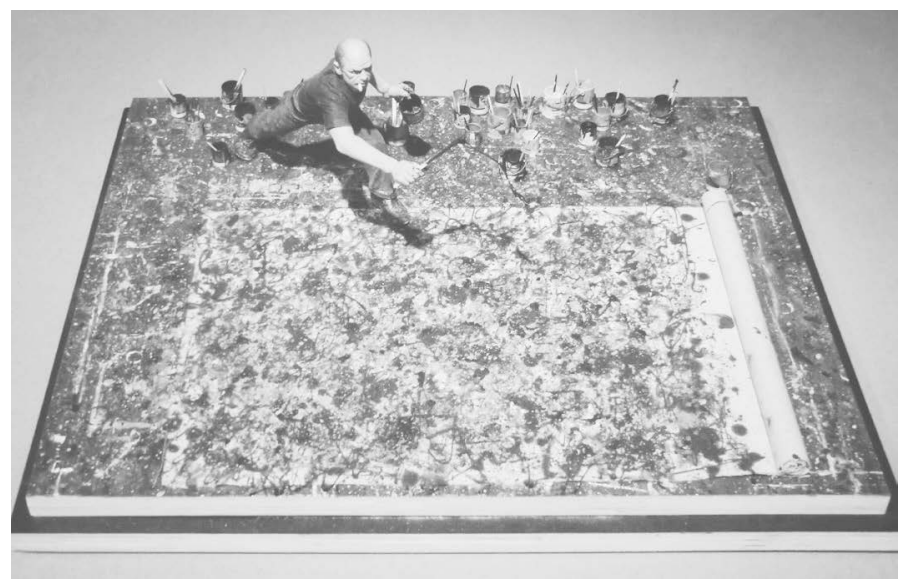

1 Joe Fig, Pollock (2008), mixed media

wie ein Tempelsockel, der das Geschehen darauf aus der Umgebung heraushebt. Was auf dem Sockel geschieht, ist der Welt Drumherum durch zwei Stufen entzogen. Der Sockel begrenzt den Betrachtungs- und Aktionsraum. Darauf befinden sich außer dem Künstler nur noch die Mittel, die für das Arbeiten des Künstlers in seinem Medium notwendig sind: Leinwand, Farben, Pinsel und Stöcke.

Pollock scheint in seiner Tätigkeit völlig auf diesen Aktionsraum konzentriert. Die Skulptur stellt einen Moment dar, in dem er von einem bestimmbaren Punkt aus Farbe in Richtung der vor ihm liegenden Leinwand wirft. Fig stellt dabei den Moment in Pollocks Malweise dar, in dem er bewusst die Kontrolle abgibt. Bis hierhin weiß er, was er tut. Er nimmt mit einem Pinsel oder Stock und schwarze Farbe direkt aus einer Dose und schleudert sie gezielt auf die Leinwand. Das Ergebnis dieser Aktion lässt sich zwar beschreibend vorhersagen - die Farbe wird auf die Leinwand treffen und sich dort in Form von Schlieren, Spuren, Tropfen und Spritzern verteilen. Pollock gibt dem Farbwurf Richtung und Geschwindigkeit. Wo genau die Farbe auftrifft und wie sie sich genau verteilen wird, kann der Künstler jedoch nicht wissen. Der Möglichkeitsraum ist zwar begrenzt, aber dadurch nicht weniger komplex.

Die Leinwand ist von einer Rolle abgerollt und noch nicht abgeschnitten worden. Das suggeriert, dass noch nicht entschieden ist, ob diese vorliegende Version ein ,Werk' ist, oder als geschei- 
terter Versuch endet, in welchem Falle der nächste Abschnitt der Leinwand abgerollt und von vorne begonnen werden könnte. Möglich ist auch, dass hier schon vorab mehrere Versuche geplant sind oder mehrere Arbeiten nacheinander produziert werden sollen. Jedenfalls weist es darauf hin, dass hier nicht an der einen Lösung gearbeitet wird, sondern eine von vielen möglichen Lösungen am Entstehen ist.

Aus drei Aspekten setzt sich der Rahmen für das Experiment zusammen, bei dem Joe Fig Pollock darstellt: I) Reduktion ist die Bedingung, die den 2) Wurf ins Ungewisse ermöglicht, dessen Ergebnis 3) unscharf bleibt, indem es eine von vielen möglichen Optionen darstellt. Im Unterschied $\mathrm{zu}$ naturwissenschaftlichen Experimenten, deren Resultate unter gleichen Bedingungen sich immer wieder einstellen müssen, sind solche gestalterischen Experimente grundsätzlich nie falsi- oder verifizierbar. Hierbei geht es nicht um den Beweis einer Hypothese, nicht um die eine Lösung für ein definiertes Problem. Hier bildet das Experiment ein gestalterisches, ergebnisoffenes Möglichkeitsfeld.

Beschränkung für Freiheit Oswald Mathias Ungers bezeichnete die Rahmenbedingungen für seine „Wochenaufgaben“ als Bindungen, durch die die Freiheit nicht eingeschränkt, sondern die im Gegenteil die Grundbedingung für Findungen sind, indem sie gleichzeitig einen Rahmen setzen, innerhalb dessen alles möglich ist. ${ }^{I}$ Reduktion steht hier also für Freiheit.

Ein solches Abstecken eines überschaubaren Experimentierfeldes bildet einen wichtigen Teil unserer Einführung in das Entwerfen im ersten Jahr des Bachelor-Studiengangs Architektur an der HafenCity Universität Hamburg. Im Verlauf des ersten Semesters wechseln sich intuitive gestalterische Aufgaben jeweils mit einer analytisch-reflektierenden Aufgabe ab. Dieser Wechsel zwischen unterschiedlichen Arbeitsmodi, der das Entwerfen als Gestaltungsdisziplin von zum Beispiel der Planung unterscheidet, wird von einer bewussten Beschränkung der verwendeten Mittel und Medien begleitet. So dienen beispielsweise in einer Aufgabe die analytisch gezeichneten Grundrisse und Schnitte des eigenen Entwurfs als Ausgangsmaterial für ein Experiment. Die Studierenden zeichnen ihre handgezeichneten Pläne zuerst in einem Zeichenprogramm nach. Anschließend kopieren, rotieren und transformieren sie selbstgewählte Elemente und setzen sie neu zu einer 


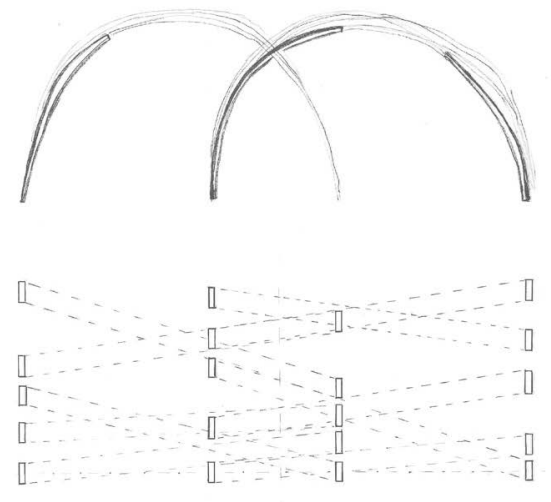

2 Grundriss und Schnitt als Ausgangszeichnung. Studierendenarbeit von Katja Hentschel, Wintersemester 2018/19, HafenCity Universität Hamburg

Zeichnung zusammen. Dieser gesetzte Rahmen bildet hier den Sockel in der Ebene der unendlichen möglichen Transformationen einer Zeichnung. Er beschränkt sie auf einige wenige ,erlaubte“ Operationen und auf das Medium der Zeichnung mit vorgegebener Zeichenkonvention und Blattformat. In einer Folge von Versuchen sollen immer wieder die intuitiv entstandenen Zeichnungen nach rein gestalterischen Qualitäten wie Regelmäßigkeit, Rhythmus, Dichte, Dynamik, Ähnlichkeit, Richtung, Beginn und Ende, Horizontalität und Vertikalität ausgewertet werden. Nach dem intuitiven Arbeiten folgt also ein Schritt der Evaluation, aus dem sich bestimmte zeichnerische Strategien ergeben, die in den nächsten Versuchen genauer und konsequenter angewendet werden sollen. Aus diesen wählen die Studierenden schließlich die besten eigenen Arbeiten aus und präsentieren sie als in Linienstärken gewichtete Schwarzweiß-Zeichnung auf vorgegebenem Blattformat. Wie das abgebildete Beispiel zeigt, entsteht dabei aus den Projektionszeichnungen einer einfachen räumlichen Struktur (Abb. 2) eine komplexe und dynamisch-grafische Komposition (Abb. 3).

Während die Einfachheit und Behäbigkeit in Abbildung 2 von einem rational-konstruierenden Dokumentationsprozess zeugen, zeigt Abbildung 3 das Ergebnis eines intuitiven Vertiefungspro- 


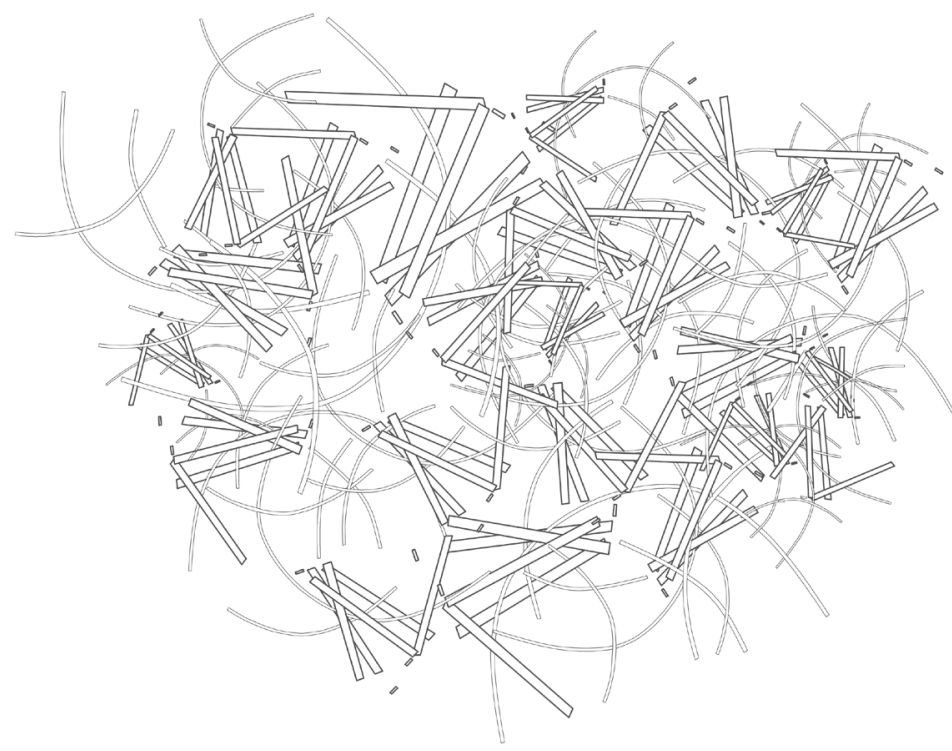

3 Ergebnis des Transformationsexperiments. Studierendenarbeit von Katja Hentschel, Wintersemester 2018/19, HafenCity Universität Hamburg

zesses, innerhalb dessen ein deutlich höheres Maß an reizvoller Komplexität, Dynamik und räumlicher Tiefe entstehen konnte. Ein wesentlicher Unterschied besteht zwischen einem vorgegebenen Endprodukt in der Aufgabe, die zu Abbildung 2 führt und einem ergebnisoffenen Prozess, der in Abbildung 3 mündet. Während die Projektionszeichnungen - hier Grundriss und Schnitt - einen Entwurf nach bestimmten Zeichenkonventionen rundum vermessen und erklären soll, ist im Transformationsexperiment kein eindeutig bestimmbares Evaluierungssystem vorgegeben. Gestalterische Kriterien werden genannt, sind aber für die Studierenden interpretations- und damit aneignungsoffen. Bewegen sich die Studierenden im Rahmen des gesteckten medialen Untersuchungsrahmens (digitale Linienzeichnung, begrenzte Transformationsoperationen), dann müssen sie über Sinn und Unsinn ihrer Entscheidungen nicht nachdenken. Es ist erst einmal alles erlaubt. Umso mehr, weil das Arbeiten in Varianten vorgegeben ist und 


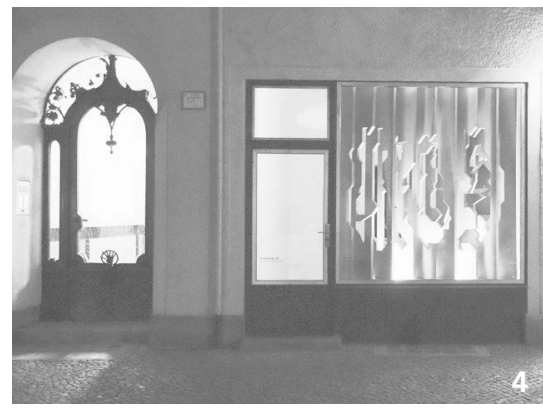

Diagonal 09 (2018)

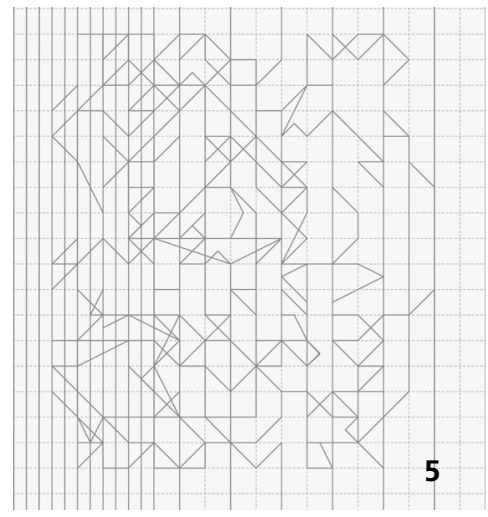

5

4 Installation des Autors im B-L Schaufenster in Berlin

5 Entwurfsraster der Installation

6 Installationsansichten

dadurch mehrere Versuche nicht nur möglich, sondern erwünscht sind. Die Studierenden sind auf ihre Intuition angewiesen, um Qualitäten zu entwickeln. Die Beschränkungen des experimentellen Setups sind in diesem Sinne die Bedingung für die erfahrene gestalterische Freiheit der Architekturstudierenden. Dieses paradoxe Verhältnis zwischen Beschränkung und Freiheit ist für diese Entwurfsmethode charakteristisch.

Experimente als Zwischenzustand Die Einführung dieser Art von Bindungen in der Grundlagenlehre des Entwurfs basiert auf der eigenen Praxis, in der ich häufig die gleiche Methode anwende. Meist dann, wenn es im Entwurf um die Entwicklung einer architektonischen Sprache durch ein gestalterisches Probieren, Erkunden und Deklinieren geht. Mit der Installation Diagonal o9, die im B-L Schaufenster Ende 2018 ausgestellt war, wollte ich eine intensive Erfahrung von räumlicher Tiefe herstellen, die sich selbst genügt. Es entstand eine „Installation zum Betrachten im Vorbeigehen“" im Schaufenster des Ausstellungsraumes, die mit den Mitteln der Parallaxe und des vielschichtigen Raumes arbeitet (Abb. 4). Die sich regelmäßig wiederholenden Bildschichten, die wie dicke Passepartouts nach ähnlichen Prinzipien ausgeschnitten sind, bilden durch die vielfältigen dadurch entstehenden Vorder-, Mittel- 


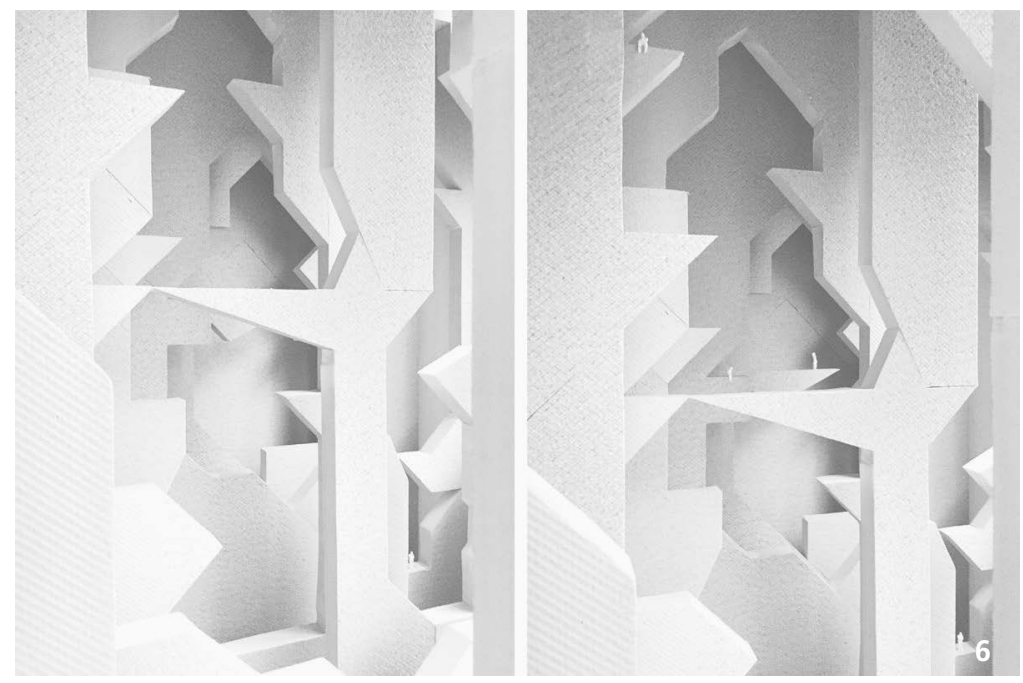

und Hintergründe einen komplexen Raum. Sie stehen diagonal, also im $45^{\circ}$-Winkel zur Schaufensterscheibe. Beim Vorbeigehen verschieben sie sich parallaktisch in der visuellen Wahrnehmung des Betrachters, was eine starke Tiefenwahrnehmung bewirkt. Innerhalb des Entwurfsprozesses entwickelte sich hauptsächlich aus den Bindungen, die das Material und die Abmessungen des Schaufensters mit sich brachten ein starres $8 \times 8 \times 8$-ZentimeterRaster in drei Dimensionen, das die Grundlage für die Entwicklung der hintereinanderliegenden Bildschichten und Ausschnitte bildete. Abbildung 5 zeigt die übereinandergelegten Projektionen der Schichten der endgültigen Version. Sie ist titelgebend und bezeichnet mit dem Index Diagonal og die Anzahl der Versuche und getesteten Varianten. Sie zeigt außerdem, wie Ausschnittformen in der Regel orthogonal oder diagonal im $45^{\circ}$-Winkel verlaufen und, dass es dabei zahlreiche Ausnahmen gibt. Die Frage, wie es zur Entscheidung kam, dass Version o9 die endgültige Version werden sollte, kann ich nicht abschließend beantworten. Sie hat sich in dem durch das Raster stark gebundenen Prozess durchgesetzt. In einem repetitiven Wechsel zwischen physischem Modell, digitaler Zeichnung und digitalem Modell schränkten sich Operationen und Materialien immer mehr ein und bildeten den beschriebenen ,Sockel ${ }^{*}$ des Möglichkeitsraumes, aus dem sich die der Installation eigene 
Sprache und Logik entwickelte. Systematisch einerseits, willkürlich andererseits: Innerhalb des gegebenen, vorbereiteten Systems wird das Medium transparent. Das heißt, obgleich es einen systematischen Rahmen setzt, wird es im Schaffen nicht mehr explizit wahrgenommen, sondern als Freiraum erfahren, in dem die Gestaltung situativ und intuitiv stattfindet.

Sowohl die studentische Zeichnung als auch Diagonal og sind nicht als Architekturprojekte zu verstehen. Sie sind nicht direkt in gebaute Projekte umsetzbar. Sie fungieren eher als Grundlagenforschung, die eine spezifische architektonische Sprache entwickelt und verfügbar macht. Die Zeichnung der Studentin wird in der darauffolgenden Aufgabe wiederum weiter interpretiert, verräumlicht, weiter transformiert und führt schließlich zu einem architektonischen Projekt. Ebenso beschreibt Diagonal og einen Zwischenzustand, der sowohl als Architektur, aber auch als Objekt an sich gelesen werden kann. Die Maßstabsfiguren (Abb. 6) sind erst auf den zweiten Blick als solche zu erkennen und verändern schlagartig die Wahrnehmung des Raumes. Die Installation wird von einem I: I Maßstab in einen Modellmaßstab versetzt. Ein architektonischer Raum wird vorstellbar.

Generative und speichernde Medien Das spanische Architekturbüro Ensamble Studio ist bekannt für seinen unmittelbaren und hemdsärmeligen Umgang mit Material und Masse und die daraus entstehenden kraftvollen Projekte - sowohl Gebäude wie auch Räume, Pavillons und Skulpturen. Immer wieder wird im Entwurfsprozess experimentiert. Bei dem Projekt Towers of Landscape - Melted Tower gingen die Architekten der Idee nach, wie natürlich geformte Räume z.B. Canyons, Klippen und Höhlen, von denen wir uns ihrer Einschätzung nach von Natur aus angezogen fühlen, mit den industriell hergestellten Architekturformen in der Großstadt reagieren könnten. ${ }^{2}$ In der Studie entstanden vier Serien von Hochhausmodellen aus Beton für die Architekturbiennale 2017 in Orléans. Die Serien entstanden nach einem klaren experimentellen Setup. Alle Turmmodelle waren gleich groß und wurden auf vier unterschiedliche Arten experimentell entwickelt. Eine der Serien ist auf der Webseite in einem für das Architekturbüro typischen Film dokumentiert. Es ist zu sehen, wie Styroportürme mit einer Art Schweißbrenner abgeschmolzen werden und so die Negativform entsteht, die in dem in kubischen Außenformen 

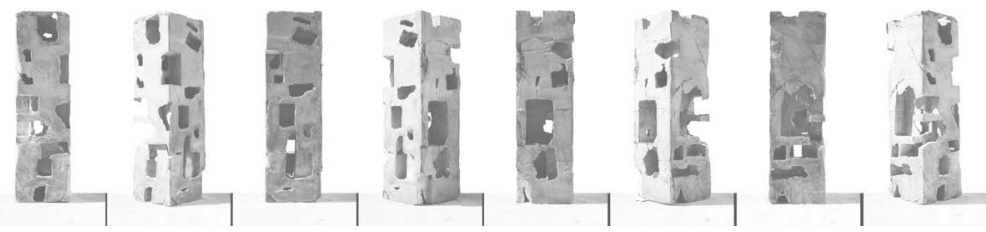

7 Ensamble Studio: Serie aus Melted Tower, 2017

gegossenen Turm anschließend die zu canyonartigen Innenräumen werden (Abb. 7).

Auch hier entsteht durch eine Entwurfsstrategie und eine ihr entsprechende mediale Beschränkung ein experimenteller Möglichkeitsraum für einen ergebnisoffenen Gestaltungsprozess. Auch hier entsteht nicht die eine Lösung, sondern eine Serie.

Die verwendeten Medien, sei es wie in den angeführten Beispielen die digitale Zeichnung oder das physische Modell wirken dabei insofern generativ, als dass in ihnen etwas entsteht, das vorher in konkreter Form noch nicht gedacht werden konnte. Je nach ihrem jeweiligen medialen Potential ermöglichen sie ein bestimmtes, zuvor unbekanntes Ergebnis. Eine Serie als Ergebnis von Wiederholungs- und Variationsversuchen dient dabei dazu, die Grenzen der entstehenden räumlichen Sprachen auszuloten und wiederkehrende Gemeinsamkeiten einer Grammatik identifizieren zu können. Die Serie bleibt nicht illustrativ in dem Sinne, als dass sie nur das Ergebnis abbildet. Sie ist gleichzeitig Endund Ausgangspunkt. Sie speichert das im Experiment entstandene Wissen über eine räumliche Sprache und deren Grammatik in ihren Objekten. In ihrem unscharfen und dadurch offenen Zwischenzustand ermöglicht sie wiederum neue architektonische Interpretationen, Lesarten und initiiert dadurch weitere Transformationen.

Kontrollverlust Nach den Beschreibungen der Bedingungen für ein intuitives Arbeiten, dem experimentellen Setup, der aus der wohlüberlegten Einschränkung des Untersuchungsraumes und der präzisen Auswahl der verwendeten Medien und den Regeln ihrer Benutzung besteht und der Beschreibung der Natur der Ergebnisse der Experimente muss es nun darum gehen, was während des Prozesses selbst geschieht: um den kontrollierten Kontrollverlust. 
Zur Eröffnung ihrer experimentellen Architekturschule Ciudad Abierta auf einem endlosen unbebauten Grundstück in der Nähe von Valparaiso trugen die Gründer Hölderlins Gedicht Der Gang aufs Land vor, das mit den Worten „Komm! ins Offene, Freund!“ beginnt. ${ }^{3}$ Eine Einladung zum Schritt ins Ungewisse, der dem Gestalter abverlangt, sich fallenzulassen und offen dafür zu sein, was das Kommende bringen wird. Beim Experimentieren geht der Gestalter ein Spiel ein. Er befindet sich mit dem Medium und den gesetzten Benutzungsregeln auf Augenhöhe. Er gibt die Kontrolle ein Stück weit an das Medium ab und wird zu dessen Werkzeug, um sie dann wieder zu übernehmen und auf das Entstandene reagieren zu können. Es entsteht ein Dialog zwischen Gestalter und Medium, in dem der gesteckte Möglichkeitsraum ausgereizt und erkundet werden kann.

In allen beschriebenen Beispielen gibt es kein ,wicked problem“ zu lösen. ${ }^{4}$ Der Gestalter steht nicht vor einer unlösbaren Aufgabe. So wie Jackson Pollocks Arbeiten keine Lösungen sind und sein wollen, so sind auch die Findungen eines Entwurfsexperiments keine Lösungen. Sie sind vielmehr Auszüge aus dem Reichtum der räumlichen Sprache, die wir implizit besser verstehen und sprechen, als im Bewussten. Die Grenzen des bewussten Gestaltens werden auch oft an produktiven Fehlern im Entwurfsprozess deutlich. ${ }^{5}$ „Ich mag dieses - wahrscheinlich ist es ein Fehler" - so oder so ähnlich kommentierte Frank Barkow oft die alternativen Vorschläge, als ich bei Barkow Leibinger an Wettbewerben arbeitete. Er hatte sich fast daran gewöhnt, dass die besten Ergebnisse zufällig und nicht intentional entstanden. Von einem ähnlichen Beispiel berichtet Philip Ursprung, wenn er beschreibt, wie im Büro von Herzog \& de Meuron der entscheidende Impuls für die Gestaltung der Fassade des Prada Flagship-Stores aus einer sozusagen unsachgemäßen Behandlung des Modellbaumaterials resultierte: „Den entscheidenden Ausschlag gab ein Modell, dessen Oberfläche mit einem Lötkolben so erhitzt wurde, dass Blasen und Beulen entstanden. Bau und Hülle ließen sich nicht mehr trennen, der Bau wird zum unendlich facettierten Schaufenster, welches den Blick nach innen ebenso wie nach außen lenkt: ,Wir haben dann das Modell geschaffen, das wir immer noch am meisten mögen. Es sieht aus wie eine Art Einkaufstüte, oder wie ein Kristall aus Plastik, mit vielen Blasen. Es gefällt uns, weil es diese Internati- 
onal Style-Glasfassaden hinter sich zurücklässt, die immer nur das gleiche zeigen" ". 6

Das neue, unverhofft Andere, entsteht erst in einem mehr oder minder gewollten Loslassen und Zulassen. Erst der temporäre Kontrollverlust ermöglicht unvorhersehbare neue Findungen. Und erst hier ist das Experiment als Begriff gerechtfertigt, indem es bezeichnet, wie nach etwas Unbestimmten gesucht wird, das vorher nicht vorstellbar und allenfalls in groben Zügen erahnt werden konnte. Anni Albers schreibt dazu: „Es lehrt den Prozess allen Schaffens, das Formen aus dem Formlosen. Wir lernen daraus, dass kein Bild existiert, bevor es gemacht wird, keine Form, bevor sie geformt wird. Die Konzeption eines Werkes gibt ihm nur seine Stimmung, nicht seine Beschaffenheit. Die Dinge nehmen im Material und im Prozess der Bearbeitung Gestalt an, und keine Vorstellungskraft ist groß genug, um vor der Ausführung der Arbeiten zu wissen, wie sie sein werden“".7

Raum als Sprache Die hier vorgestellte Methode des experimentellen Entwerfens ist eine Art Trick, um innerhalb eines komplexen und meist von diskursiver Argumentation dominierten Entwurfsprozesses einen Wirkungsraum für die Intuition zu schaffen. Das Experiment ermöglicht, sich vorübergehend aus dem komplexen Kontext zu lösen, um intuitiv eine räumlich-gestalterische Sprache zu finden. Es entspricht dem naturwissenschaftlichen Experiment, in dem ein bestimmtes Phänomen isoliert und in kontrollierten Laborbedingungen untersucht wird. Auch bei diesem Verständnis eines Experiments ist die Reduktion eines Phänomens nicht absolut, sondern geschieht nur rein methodisch zum Zwecke der Untersuchung. ${ }^{8}$ Und wie beim gestalterischen Experiment muss sich das Ergebnis wieder in den ursprünglichen komplexen Zusammenhang einfügen. Wie bei den aufgeführten Beispielen beschrieben, entstehen hier keine Lösungen, sondern Sprachen. Die Ursprungsfrage von Ensamble Studio, wie canyonartige, höhlenartige Räume mit den Gebäudegeometrien der Stadt verbunden werden können, führte $\mathrm{zu}$ dem oben beschriebenen experimentellen Setup. Die darin stattfindende, intuitive, aber systematische Forschung folgte dem Ziel, Wissen zu schaffen - sie ist also eine wissenschaftliche Forschung. Und eine notwendige. Denn von der Idee, canyonartige Räume bauen und erfahrbar machen zu wollen bis zu dem Wissen, wie eine solche Grammatik des Raumes funktionieren 
kann, ist es ein weiter Weg. Dessen erster Schritt war der gezielt gesteckte Untersuchungsraum des Experiments, das experimentelle Setup, der Sockel. Dieser ist so gesetzt, dass eine Verwendbarkeit der Ergebnisse wenn nicht sicher, so doch wahrscheinlich ist. Aus den experimentellen Untersuchungen lässt sich die räumlich gestalterische Grammatik extrahieren. Sie bildet den Ausgangspunkt für Fragen, wie sich solche Formen im Gebäudemaßstab herstellen und planen lassen, welche Nutzungsoptionen sie zulassen und unter welchen Bedingungen. Und schließlich, sollte sie dann realisiert werden, sind die Experimente der Ursprung für die Gestalt des Raums, in dem sich menschliche Körper bewegen und in Beziehung zueinander und zur Architektur treten und damit in die Sprache einstimmen können, die von der Architektur angestimmt wird. Dabei verkörpert diese Sprache im Medium Raum diejenigen Themen, Ideen und Inspirationen, die zu ihrer Entstehung geführt haben. Sie erklärt sie nicht, sondern sie macht sie erfahrbar. Sie dient als Gesprächspartner für die unmittelbare Wahrnehmung und vermittelt eine spezifische, einzigartige räumliche Erfahrung.

Intuition und Autorenschaft Mit der Untersuchung des ,creative flow" genannten Phänomens nähert sich die Psychologie den Bedingungen und Merkmalen der hier beschriebenen kreativen Prozesse. Begrifflichkeit und Definition basieren auf dem von Mihaly Csikszentmihalyi eingeführten Beschreibung des ,flowZustands". 9 Diese ursprünglich auf das Empfinden von Glück ausgerichtete Untersuchung wurde unter dem Begriff des ,creative flow" auf bestimmte kognitive Zustände im kreativen Prozess von Gestaltern - bislang Malern, Komponisten und Schriftstellern - erweitert. Interviews mit Künstlern führen zu einer Beschreibung der Elemente, die diesen Zustand charakterisieren. Unter anderem umfassen diese das Heraustreten aus der Alltagsroutine in eine andere Realität, das Verschmelzen von Aktion und Aufmerksamkeit, Spontanität, Verlust des Zeitgefühls, Absorption zusammen mit einer Unsicherheit der verfolgten Ziele, der Möglichkeit, von den Ergebnissen überrascht zu werden. Nach Charlotte L. Doyle finden sie ihre Entsprechung in der Beschreibung des ,Type I cognitive process“ in der sogenannten „Dual Process Theory“, die zwei unterschiedliche kognitive Verarbeitungswege unterscheidet. Dabei wird das intuitive Denken als schneller und leistungsstarker kognitiver Verarbeitungsweg (Type I) vom reflektierten Denken als 
dem unbewussten, kontrollierten, langsamen, leistungsschwachen Verarbeitungsweg (Type 2) unterschieden. Der „creative flow“, so vergleicht Doyle weiter, weise dazu Eigenschaften sowohl von konvergenten als auch divergenten Denkprozessen auf. Das konvergente Denken zielt auf eine einzige, richtige Lösung, während beim divergenten Denken mehrere Möglichkeiten in unterschiedlichen Richtungen gesucht werden. ${ }^{\text {Io }}$

Das entspricht den eigenen oben ausgeführten Beobachtungen, die ein Wechsel zwischen Kontrollverlust und -übernahme beschrieben haben. Auch beim eingangs angeführten Beispiel von Jackson Pollocks Arbeitsweise finden sich Parallelen. Die Skulptur in Abbildung I zeigt ihn mit einem Fuß auf der bearbeiteten Leinwand, die Zigarette im Mundwinkel, die dann als Stummel in das Bild eingearbeitet werden wird. Manisch arbeitend, im Ausnahmezustand, im Rausch. Gleichzeitig wurde bei der Restauration seiner Arbeiten entdeckt, dass bestimmte Farbschichten in späteren Arbeitsschritten gezielt überdeckt wurden. ${ }^{\text {II }}$ Das weist darauf hin, dass neben oder im rauschhaften Malen auch zielgerichtete Intentionen verfolgt werden und eine aktive, bewusste Autorenschaft stattfindet. Der Autor wird in diesem Prozess nicht unsichtbar und unbedeutend. Oder wie der Bühnenbildner Bert Neumann es formuliert: „Ohne die künstlerische Autonomie jedes Einzelnen auch und gerade im kollektiven Arbeiten wird man den beglückenden Moment der Entstehung von Kunst nicht erleben“. ${ }^{12}$

Diese Beobachtungen scheinen paradox, da ja gerade der empfundene Verlust der Kontrolle innerhalb des gestalterischen Experiments der Vorstellung von Autorenschaft zu widersprechen scheint. Sie beschreiben einen unbewussten Autor, der sich selbst als ein Medium einem intuitiven modus operandi öffnet und ein Werk, das in diesem Prozess erst seine Eigenständigkeit entwickelt. Und dabei ist es offenbar, dass die Bedingung für die ungestörte Innerlichkeit des intuitiven kreativen Prozesses ein autonomer Autor ist. In einem Interview bezeichnet Juhani Pallasmaa die Entstehung seiner Texte als Kollaboration mit der eigenen Arbeit. Er beschreibt dabei, wie die Abgabe von Kontrolle an das Medium der Moment ist, indem eine spezifische Qualität entsteht: „Die ultimative Fähigkeit ist die Zusammenarbeit mit der eigenen Arbeit, nicht so sehr die mit anderen. Ab einem bestimmten Punkt erhält das Werk, ob Entwurf oder Text, sein eigenes Selbst. Das ist es, was du entdecken musst. Dann übernimmt es die Führung 
und beginnt sich zu behaupten. Ein toller Moment. Ich schreibe meine Essays immer in acht bis zehn Runden zwischen mir und meiner Sekretärin. Bis etwa zur vierten oder fünften Runde habe ich das Gefühl, dass ich schreibe, und von da an ist es der Aufsatz, der mich schreibt. Es ist ein wirklich genussvoller Moment, wenn die Sache die Oberhand gewinnt und beginnt, Ideen vorzuschlagen - immer die besten Ideen. Für mich ist dieser Moment in einem kreativen Prozess mit Atmosphäre verbunden. Das ist der Punkt, an dem das Werk eine Atmosphäre an sich, eine Einheit und einen eigenen Charakter erhalten hat. Es beginnt, sich auf dich zu projizieren. Du stützt deine Entscheidungen für die nächsten Schritte und Details auf dieses Gefühl der Atmosphäre, nicht auf eine theoretische Sichtweise oder auf Vorurteile. So scheint es, dass in einem kreativen Prozess die Atmosphäre in diesem Moment, in dem die Arbeit übernimmt, in dem die Arbeit Persönlichkeit erlangt, unabhängig von sich selbst in das Werk eintritt". ${ }^{13}$

Conclusio Die hier beschriebene Methode des experimentellen Entwerfens ist mit Sicherheit nicht der einzige Weg, wie ein intuitives Gestalten möglich wird. Es sollte jedoch deutlich geworden sein, dass Intuition in der architektonischen Gestaltung eine der wesentlichen Quellen von neuen Findungen ist, indem sie nichtdiskursiven und nicht-reflektierten Gedanken Ausdruck ermöglicht. Gestalterische Experimente solcher Art ermöglichen es so, Themen und Phänomene in räumliche Sprache zu transferieren und dadurch in die Architektur einzuschreiben. Architektur selbst ist damit ein Medium der Verkörperung. Die nicht-sprachliche, nicht-diskursive, unscharfe Kommunikation durch Raumerfahrung ist die Dimension, die Architektur im Kern zu einer kulturellen und künstlerischen Disziplin macht.

Wie die Beispiele gezeigt haben, benötigt Intuition einen eigenen, abgelösten Raum, in dem diskursive Argumentationen ausgeschlossen sind. Ein solcher Raum bringt Autor und Medien auf einer intuitiven Ebene zusammen und ermöglicht dem Gestalter den Schritt ins Offene. Und indem die Medien darin von ihrer illustrativen Aufgabe befreit als Gestaltungsmittel per se funktionieren, werden sie zu einem intuitiven und generativen Interaktionspartner des Gestalters. 
Anmerkungen

1 Erika Mühlthaler, "Lernen von O.M. Ungers", Arch+181/182, 2006, S. 75.

2 "Whether it is their roughness, their wilderness, their low resolution, their continuous transformation, their connection with the essential and disconnection with urban environments, their lack of determination that liberates the spirit and excites the imagination... or all of the above, we are inherently attracted to such spaces, structures, images". Ensamble Studio: Towers of Landscape. Orléans Architecture Biennial, France. Melted Tower, 2017, www. ensamble.info/towers-of-landscape [26.03.2019].

3 Sebastian Redecke, „Mit eigener Kraft. Ciudad Abierta Ritoque Campamento Mesana", Bauwelt 3, 2015, S. 14-23, hier: S. 16.

4 Als „wicked problems” bezeichnet Horst Rittel die Aufgaben der planerischen Disziplinen. Er beschreibt dadurch die Natur der Entwurfs- und Planungsaufgaben, die keine logische Lösung ermöglichen, da sich die Anforderungen teilweise widersprechen, sich ändern und unter Umständen auch unbekannt sind. Vgl. hierzu u.a.: Horst W. J. Rittel, Melvin M. Webber: „Dilemmas in a General Theory of Planning", Policy Sciences 4, Nr. 2 1973, S. 155-169.

5 Erste empirische Untersuchungen zur Überlegenheit intuitiver Evaluation von Situationen finden sich bei: Charles S. Peirce, Joseph Jastrow: „On small differences of sensation”, in: National Academy of Sciences (Hg.), Memoirs of the National Academy of Sciences, Bd. 5, Washington 1884, S. 75-83. Aktuell wird Intuition in kreativen Denkprozessen auch in ihrer Rolle im kognitiven Prozess des „problem solving” untersucht. Siehe dazu u.a.: Kenneth J. Gilhooly, "Incubation and Intuition in Creative Problem Solving", Frontiers in psychology 7, 2016, S. 1-9.

6 Philip Ursprung, „Exponierte Experimente. Die Modelle von Herzog \& de Meuron", in: Oliver Elser, Peter Cachola Schmal, Philipp Sturm u.a. (Hg.), Das Architekturmodell, Frankfurt a. M., Zürich 2012, S. 51-56, hier: S. 56.

7 Anni Albers, "One Aspect of Art Work", in: Brenda Danilowitz (Hg.), Anni Albers, Middleton 2000, S. 25-26. Im Original auf Englisch: "It teaches the process of all creating, the shaping out of the shapeless. We learn from it that no picture exists before it is done, no form before it is shaped. The conception of a work gives only its temper, not its consistency. Things take shape in material and in the process of working it, and no imagination is great enough to know before the works are done what they will be like." Übersetzung durch den Autor. 
8 Vgl. hierzu: Christopher Alexander: The nature of order. An essay on the art of building and the nature of the universe, The Phenomenon of Life, Berkeley 2002, S. 16.

9 Mihaly Csikszentmihalyi, Das Flow-Erlebnis. Jenseits von Angst und Langeweile, im Tun aufgehen, Stuttgart 2010.

10 Charlotte L. Doyle, "Creative Flow as a Unique Cognitive Process", Frontiers in psychology 8, 2017, S. 1-4.

11 Janelle Zara, A Live Jackson Pollock Restoration Reveals Fascinating New Discoveries, 2018, www.galeriemagazine.com/jackson-pollockrestoration-los-angeles/ [31.03.2019].

12 Bert Neumann, Laudatio auf Förderpreisträger Andy Seno Aji, 2015, www.hein-heckroth-ges.de/2015 [31.03.2019].

13 Klaske Havik, Gus Tielens, Juhani Pallasmaa: „Atmosphere Compassion and Embodied Experience. A Conversation about Atmosphere with Juhani Pallasmaa", OASE 91 Building Atmosphere, 2013, S. 33-53, hier: S. 39-41. Im Original auf Englisch: "The ultimate skill is to collaborate with your own work, not so much to collaborate with others. After a certain point the work, whether design or writing, obtains its own self. That is what you need to discover. Then it takes over and begins to suggest itself. A great moment. I always write my essays in eight to ten rounds between me and my secretary. Until about the fourth or fifth round I have the feeling that I am writing, and from there on it is the essay that is writing me. It is a really pleasurable moment when the thing takes over and begins to suggest ideas - always the best ideas. To me, this moment in a creative process is associated with atmosphere. That is the point where the work has obtained an atmosphere in itself, a unity and a character on its own. It begins to project on you. You base your decisions for next steps and details on that hunch of atmosphere, rather than on a theoretical view or on preconceptions. Thus, it seems that in a creative process, atmosphere enters the work in this very moment when the work takes over, when the work achieves personality, independently of yourself." Übersetzung durch den Autor. 
Norbert Palz

\section{Architektonischer Entwurf und differenzierte Mediennutzung}

Einleitung Wissenschaftliche Innovationen entstehen in den seltensten Fällen als gänzlich originäre Erfindungen, sondern sind oftmals das Ergebnis einer kreativen Neubetrachtung und Montage zuvor getrennter Erkenntnisse in neuen Verfahren oder eigenständigen Technologien. Diese schöpferische Rekombination ${ }^{\mathrm{I}}$ vereinigt (fragmentarische) Erkenntnisse aus vielen Bereichen und Zeiten in einer neuen dynamischen Wissenslandschaft, die sich in der Folge wieder für einen neuerlichen Prozess der Wissensgewinnung und Innovation öffnen kann. Diese Perspektive auf innovatives Handeln betrachtet historisches und gegenwärtiges Wissen als - potentiell - wirksame Einflussgrößen, die ein zukunftsgerichtetes gestalterisches Handeln formen können. Implizit zu dieser Haltung ist ein Verständnis von Geschichte, das nicht als Sequenz abgeschlossener - tendenziell linearer - Narrative begriffen wird, sondern als reflexives und schöpferisches Element eines Gestaltungsvorgangs.

Eine besondere Rolle kommt hierbei den operativen, medialen und instruktiven Schnittstellen gestalterischen Handelns zu, haben sie doch dafür Sorge zu tragen, dass formgebende Informationen in eine les- und vermittelbare Form überführt und transformiert werden können. Die Architektur hat in der Vergangenheit verschiedenste dieser medialen Schnittstellen von großer Abstraktion entwickelt, um aus den unterschiedlichsten planerischen Parametern - seien sie technisch oder heuristisch - eine eindeutige räumliche und konstruierbare Lösung ableiten zu können. So fußten die Architekturzeichnungen der Renaissance auf den mathematischen Regeln der Darstellenden und Projektivgeometrie wie sie von Gaspard Monges beziehungsweise Philibert de l'Orme niedergelegt waren. Die Universalität dieses repräsentativen Mediums, 
unabhängig gegenüber individuellen stilistischen Ausprägungen, fungierte als informative und integrierende Schnittstelle - von der Baustelle bis zum Auftraggeber - aber auch als kommunikatives Instrument innerer Vermittlung architektonischen Wirkens. Es versteht sich von selbst, dass sich trotz dieser Festlegungen immer auch eigenständige Auslegungen dieser Protokolle in Bild und Modell finden lassen, die als Signatur des Gestaltenden eine Interpretation des mathematischen und ikonographischen Raumes formulierten. Interessanter als diese individualisierten Interpretationen sind allerdings die Wechselwirkungen, die diese abstrahierten Schnittstellen im Verlauf ihrer Anwendung auf das räumlich-konstruktive Vokabular des sie anwendenden Planers ausüben und so einen reziproken Dialog zwischen Planungswerkzeug und konstruktiver Materialisierung beförderten. ${ }^{2}$ Die Möglichkeiten mathematischer Beschreibbarkeit entlang einer codierten zeichnerischen Prozessoralität bereiteten den Weg für neue räumliche Morphologien und Bauteiltypologien, die nur diesem Werkzeugkasten avancierter Geometrie entspringen konnten. ${ }^{3}$ Entwerferische Entscheidungen entwickelten sich nun aus einem Selektionsvorgang geometrischer Variantenbildung.

Ein besseres analytisches Verständnis über die Wechselbeziehungen von Material und Geometrie war ab dem I9. Jahrhundert für eine Neubetrachtung und teilweisen Ablösung tradierter Geometriesysteme verantwortlich, indem das Material selbst und seine darin eingeschriebenen strukturellen Vorgänge zunehmend in den Vordergrund einer ingenieursmäßigen Entwurfsauffassung und davon abgeleiteten neuen Raumformen rückten. Durch die experimentellen Arbeiten Heinrich Hübschs, Antoni Gaudís, Frei Ottos, Sergio Musmecis und Anderen entstanden nun aus den strukturmechanischen Eigenschaften des Materials abgeleitete Formfindungsmethoden, die unabhängig von tradierter Tektonik und zeichnerischer Darstellung operierten. Das physische Modell lenkte den Entwurfsprozess, die Darstellungsform und den impliziten morphologischen Lösungsraum, der sich aus einem emergenten Materialverhalten prozessoral herausbildete. Ein sich aus dieser Praxis entwickelnder Wandel der Bedeutung zeichnerischer Darstellung hatte auch pragmatische Gründe angesichts der geometrischen Komplexität sich bildender Materialgefüge, die mit den Hilfsmitteln der Darstellenden Geometrie nicht mehr praktikabel zu bestimmen waren. 
Gerade am Beispiel von Gaudís Hängemodell der Colònia Güell Kirche finden wir ein eigenes, zeichnungsunabhängiges vernetztes physisches Entwurfsmedium, das eine Position zwischen einem realen Gebäude und dessen Repräsentation einnimmt. Aber auch Heinrich Hübschs - fast hundert Jahre zuvor - entwickelte Zeichnungsinstrumente, die er für seine Formfindung zweidimensionaler druckbeanspruchter Bogenformen verwendete, basierten vornehmlich auf einem idealisierten Materialverhalten, das sich an der Hängefigur von Kettenlinien orientierte, in seinem resultierenden formalen Kanon allerdings noch stärker vom Historismus des I9. Jahrhunderts vorkonditioniert war. ${ }^{4}$ Beide Formen eines über das Materialverhalten vermittelten Entwurfsprozesses entsprangen keiner epochalen Modeströmung, sondern einer forschenden Praxis, deren Ursprünge vielfältig waren und sich aus einer individuellen Entwurfsauffassung ableiteten. Es kann spekuliert werden, dass Gaudís über Formfindungsmodelle entwickelte Gewölbestrukturen von katalanischen Mauerwerkstraditionen inspiriert waren, die für ihn als patriotischen Architekten große Bedeutung hatten und in der Folge mit seinem radikalen Ansatz zu struktureller Formfindung, handwerklicher Innovation und künstlerischen Talenten verbunden wurden. Jeder der obengenannten Architekten verfügte also über einen höchst individuellen Satz an experimentellen Planungs- und Konstruktionswerkzeugen, die einem eigenen, über Jahre entwickelten Forschungsinteresse entstammten, jedoch auch von den personellen und infrastrukturellen Konditionen der Profession und Epoche abhängig waren.

Zeitgenössische computergestützte Entwurfsprozesse erweitern das Repertoire planerischer Werkzeuge, Instrumente und Medien. Räumliche Form entsteht nun aus einem Prozess ineinander verschränkter technologischer, performativer und qualitativer Rahmenparametern, die in Verbindung $\mathrm{zu}$ prozessoralen digitalen und analogen Werkzeugen gesetzt und kalkulatorisch abgewogen werden. Das Narrativ der Formgebung leitet sich aus der elaborierten organisatorischen Komposition technischer Prozessabläufe $a b$, die in Abstimmung zu den prinzipiellen Parametern eines computergestützten Gestaltungsraumes und einer vielfältigen Herstellungstechnologie gesetzt werden. Die gestalterischen Lösungsräume werden in diesem Prozess also technologisch, prozessoral und repräsentativ so vorkonditioniert, wie es in den Bedingungen algorithmischer Beschreibbarkeit angelegt 
ist. Dieser determinierte gestalterische Handlungsraum ist jedoch auch nicht frei von potentiell manipulativen Einflussfaktoren, die die Zusammensetzung der zur Lösung angewandten Parameter bestimmen. ${ }^{5}$ Eine ganzheitlichere mediale Betrachtungsweise, die eine übergreifende Kombinatorik aller Entwurfs- und Herstellungsmedien - digital wie analog - potentiell und gleichermaßen in Anwendung imaginiert, ist allerdings innerhalb zeitgenössischer computergestützter Architekturforschung nur selten zu finden. Ein projektspezifisches Tableau hybrider Mediennutzung und zeitlich beziehungsweise inhaltlich weiter aufgespannter Themenfelder zu entwickeln erscheint sinnvoll, zu repetitiv sind doch die Resultate eines ausschließlich am Digitalen ausgerichteten Entwurfszugangs.

Ein kurzer Blick auf historische Beispiele computergestützten Entwerfens zeigt allerdings auch, dass diese Abhängigkeit von Technologie in ihrer logischen Konditionierung und Raumproduktion schon früh nachweisbar und intrinsisch mit dem Medium verbunden ist und die Rahmenbedingungen des Entwurfs immer beeinflusst hat. So konzentrierten sich die Forschungen zum computergestützten Entwerfen in den ersten Dekaden der Nachkriegszeit weitestgehend auf numerische Berechnungen einer (vermeintlich) rationalen Raumkombinatorik, die einen nicht heuristischen Planungsvorgang anleiten sollten. Begründen lässt sich dieser thematische Fokus einerseits aus der bescheidenen Funktionalität früher Rechenanlagen und anderseits aus einem politischen Interesse an einer wissenschaftlicheren Bewertung von Planungsverfahren in der Nachkriegszeit. ${ }^{6}$ Die logische Konditionierung räumlicher Relationen, durch die ein idealisiert-positivistischer, algorithmischer Entwurfsprozess ausgelöst wurde, lieferte am Ende des Rechenvorgangs abstrakte Graphen und zweidimensionale Matrizen. Die algorithmische Beschreibung typologisch kombinierbarer Raumqualitäten und Sequenzen basierte jedoch auf Festlegungen, die sich an traditionellen und vernakulären architektonischen Beispielen orientierten. Die Varianten räumlicher Relationen wurden computerbasiert bestimmt, die architektonische Übersetzung blieb formal unbestimmt und musste - wie gewohnt - in analoger Manier extrapoliert werden, zum Beispiel in Christopher Alexanders 7 Pattern Language (Abb. I) oder George Stinys Shape Grammar.

Computergestützte Entwürfe der I990er Jahre konzentrierten sich dahingegen auf die konkrete morphologische und zeitbasierte 

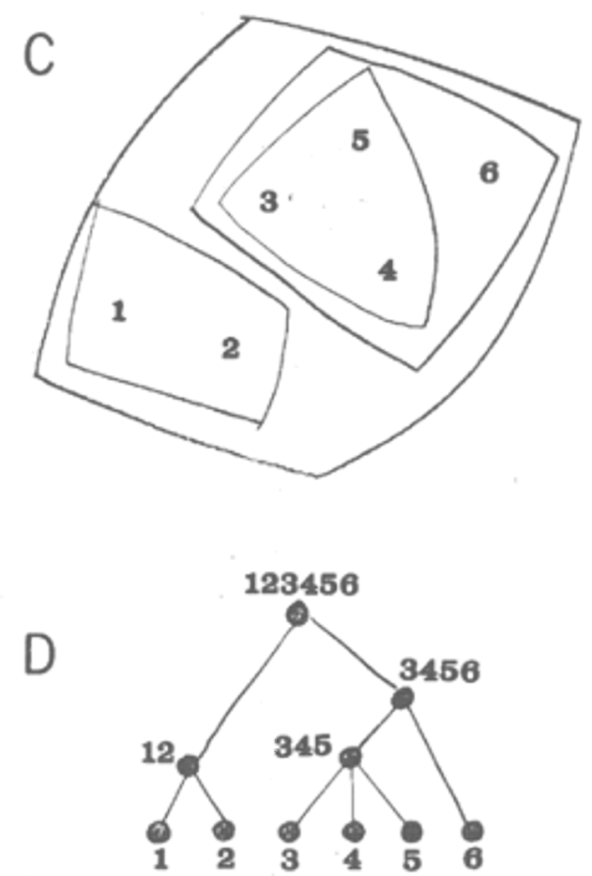

1 Christopher Alexander, Diagramm aus „A City is not a Tree"

Entwicklung einer neuen Raumgestaltung, die auf der prozessoralen Anwendung kinematischer Animationssoftware beruhte. Die dabei entstehenden spektakulären Visualisierungen und Animationen dreidimensionaler fluider Oberflächenaggregate profitierten von den Innovationen der Computergrafik, welche ab den I970er Jahren große Fortschritte in der farbigen Bildgebung von dreidimensionalen Geometriedaten erreicht hatte. Diese architektonische Avantgarde, die auch den Ursprung der aktuellen Diskurse bildet, hatte sich bewusst für einen Bruch mit den bekannten tektonischen, typologischen und konstruktiven Architekturkonzepten der vordigitalen Zeit entschieden. Diese Generation war also gerade nicht daran interessiert eine konzeptionelle Kontinuität zwischen ,historischen“ analogen Entwurfsverfahren und digitalen Arbeitsabläufen herzustellen, sondern setzte auf einen grundsätzlichen Neuanfang 
in der Beziehung zwischen Medium und Raum in direkter Korrespondenz zur Technologie.

Der Unterschied dieser beiden, zeitlich um 30 Jahre versetzten, computergestützten Entwurfskonzepte liegt auch in einer qualitativen Verschiebung der Interaktion zwischen Medium und Nutzer - vom numero-logischen zu pikto-morphologischen ${ }^{8}$ - praktischen und repräsentativen Entwurfsanwendungen, die jeweils direkt in das Denken und Handeln der Entwerfenden zurückwirkte. Diese enge, technikzentrierte Beziehung zwischen Medium, entwerfender Person und Entwurf setzt sich bis heute fort. Die Leistungsfähigkeit computergestützter architektonischer Entwurfsmedien basiert heute auf einer technisch immer avancierter und benutzerfreundlicher werdenden Hard- und Software, letzteres zusätzlich von den Aktivitäten global vernetzter Nutzergruppen profitierend, die in Form eines Bottom-Up-Prozesses an der Diversität möglicher funktionaler Programmbausteine arbeiten.

Die im Folgenden aufgeführten Fallstudien sollen beispielhaft für einen erweiterten Diskursraum stehen, der Technologie, Autorenschaft und heterogene Wissensgebiete auf neuartige Weise verbinden möchte. Warum ist dies von Belang? Es ist zu vermuten, dass architektonische Innovationen eben nicht nur in etablierten Forschungssegmenten computergestützter Gestaltung, sondern auch in deren fachlichen Randbereichen $\mathrm{zu}$ finden sind, die sich nicht sofort für eine prozessorale Verarbeitung anbieten und die sich anschicken, heterogene Wissensfelder, Praktiken und Erkenntnisse zu verbinden. Auch die bewusstere Wahrnehmung und Operationalisierung von existierendem vor-digitalem architektonischem Wissen kann sich positiv auf das gestalterische Repertoire auswirken und neue typologische, formale und strukturelle Innovationen - jenseits der Vorhersehbarkeit - erschließen. Der avantgardistische Kern der so entstehenden Architektur wird dadurch nicht berührt. Dieser konzeptionell eklektische Zugang ist hilfreich, um einen Gestaltungszugang zu stärken, der sich nicht ausschließlich nach positivistischen Kategorien ${ }^{9}$ ausrichtet, sondern eine individualisiertere projekt- und kontextspezifische Kombinatorik formrelevanter digitaler und analoger Prozessakteure versammelt.

Die Notwendigkeit, diese Wege intensiver zu beschreiten, leitet sich auch aus der systemischen Rolle von Architektur ab, ist sie doch lebendiger Knotenpunkt in einem von technologischen, soziokulturellen, räumlichen und wirtschaftlichen Parametern 
prozessoral aktualisierten und sich materialisierenden Umweltsystem. Es ist ein für die Zukunft herausforderndes Ziel, Prozesse computergestützter Gestaltung an diese Komplexität menschlichen Zusammenlebens anzupassen und sie technologisch auszuentwickeln. Reifere computergestützte Gestaltungsprozesse können nun auch Themenfelder verbinden, die über einen guten Forschungsstand innerhalb und außerhalb der Architektur verfügen. Sie finden sich beispielsweise in der Entwicklung von Gestaltungskonzepten im urbanen Kontext, in deren Materialien und Traditionen, aber auch in der Modellentwicklung für prozessorale politische, ethnische, soziale, ökologische und ökonomische Konditionen des Raums. Diese Schwerpunktverlagerung von der Technologie zum Konzept würde hiermit einer historischen Kritik Christopher Alexanders an rein von Technologie geleiteter Gestaltung entgegentreten, indem nun solche Anwendungen gefunden werden könnten, die die Konditionen kultivierten menschlichen Zusammenlebens positiv entwickeln könnten und konkreten Problemstellungen entstammen: ,Jemand, der fragt: Wie können wir den Computer auf die Architektur anwenden? ist gefährlich, naiv und dumm, denn nur eine dumme Person möchte ein Werkzeug verwenden, bevor er einen Grund für dessen Verwendung hat. Er ist naiv, denn wie uns die tausend Beamten gezeigt haben, kann ein Computer nur sehr wenig, wenn wir nicht zuerst unser konzeptuelles Verständnis von Form und Funktion erweitern. Zudem ist er gefährlich, weil uns seine Voreingenommenheit womöglich daran hindert, dieses konzeptuelle Verständnis zu lesen, sowie Probleme so zu sehen, wie sie wirklich sind."Io

Der nachfolgende Textabschnitt wird Projekte präsentieren, die für das bessere Verständnis einer solch erweiterten Praxis computergestützter Gestaltung hilfreich sein können. Sie sind in der Wahl ihrer formgebenden Informationen heterogen und in deren Wechselwirkung komplex.

\section{Verbindung heterogener Wissensgebiete durch computerge-} stützte Entwurfsmedien Die folgenden drei architektonischen Projekte demonstrieren beispielhaft die Anwendung computergestützter Gestaltungsprozesse für die Vermittlung unterschiedlicher Themen aus den Bereichen Konstruktion, Materialität und Nutzung. Die Projekte unterscheiden sich erheblich in den jeweiligen Maßstäben, der Darstellungsmethode und ihrer physischen 
Präsenz, sie teilen aber alle drei die Eigenschaft, dass sie nicht ohne technologische Infrastruktur und avanciertes Wissen in Informatik und Ingenieurwesen realisierbar wären. Sie sind also computerbasierte Projekte.

Das erste Projekt Project ColomBrick (von der Block Research Group ETH, Zürich) untersucht einen hybriden Mediengebrauch, der zwischen computerbasiertem Entwurf und traditionelleren handwerklichen Praktiken liegt. So finden digitale Verfahren zur strukturellen Formfindung von unbewehrten Gewölben aus dünnen Fliesen Anwendung, die Errichtung erfolgt über ungeschulte lokale Hilfskräfte.

Das zweite Projekt Concrete[I]land (von François Roche / New Territories / LabM4 + Michigan Ann Arbor) entwickelt ein filmisches Narrativ um einen architektonischen Entwurf, eingebettet in einen komplexen sozialen und technologischen Kontext eines thailändischen Ghettos. Eine hüttenähnliche Struktur aus additiv gefertigten Schindeln mit adaptiver Morphologie stellt den eigentlichen - fragmentarisch bleibenden - Entwurf dar, um den der Film kreist. Der Beitrag kombiniert technologische Beiträge mit ästhetischen Qualitäten und Darstellungsmethoden aus Wissenschaft und Kunst.

Das letzte Projekt Multisensory Architecture (von Sean Ahlquist, Taubman College of Architecture and Urban Planning; Leah Ketcheson, University of Michigan, School of Kinesiology; Costanza Colombi, University of Michigan, Department of Psychiatry) untersucht neue erweiterte räumliche und taktile Schnittstellen für die Förderung des Lernens und sozialen Engagements von Kindern mit Autismus-Spektrum-Störung (ASS). ${ }^{\text {II }}$ Das Projekt leitet seine geometrische Formgebung und Materialität von medizinisch-therapeutischen Funktionen ab.

Die verschiedenen Beschreibungen und Interpretationen, die auf den folgenden Seiten dargelegt werden, konzentrieren sich auf die Beziehungen zwischen den gebauten Objekten, ihrer Darstellung und ihren breiten konzeptuellen Wurzeln, von denen sie abgeleitet wurden - seien sie digitaler oder analoger Herkunft.

Projekt ColomBrick Das Projekt beschreibt Formfindung und Herstellung einer flachen, halb eingegrabenen Mauerwerksschale am Saum des Parks Juanes de la Paz in Medellin, Kolumbien (Abb. 2). Das Projekt entstand im Jahr 20 I 4 anlässlich des siebten World 


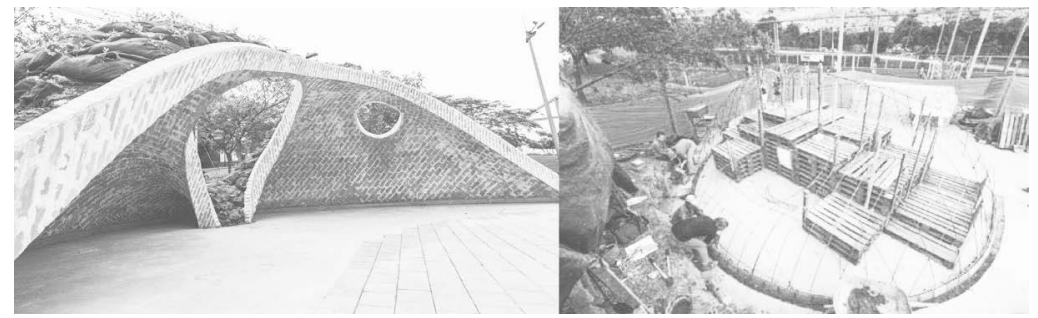

2 Links: Mauerwerksstruktur als Freiformschale, Verhandlung des städtischen Grüns und der Stadtlandschaft; rechts: Minimale Skelettschalung, die die Oberflächen zeigt, Block Research Group ETH Zürich 2014

Urban Forums und wurde von einer internationalen Kooperation ${ }^{12}$ von Architektinnen, Ingenieuren und Handwerkern entwickelt und gebaut.

Für die spezifische Situation des Grundstücks am Rande eines innerstädtischen Parks wurde eine dünne, mehrlagige Ziegelstruktur entworfen, die zwischen Stadtlandschaft und urbanem Grün vermittelt. Die Freiformschale differenziert sich in innerer und äußerer Anmutung, Materialität und Geometrie. Der ,innere“ urbane Teil der Schale, welcher sich zu einer befestigten Freifläche hin öffnet, weist eine sichtbare Ziegelstruktur mit farblich abgesetzter Mörtelfuge auf, während der zum Park orientierte ,äußere“ Bereich von einer dicken Grünschicht bedeckt wird, die sich kontinuierlich vom Park aus auf das Vordach über die gesamte Oberfläche erstreckt. Der Aufbau der Ziegelschale erfolgt hierbei in der traditionellen katalanischen Gewölbetechnik, bei der dünne Ziegel in einem mehrschichtigen Fischgrätenmuster verlegt werden.

Die Berührungslinie beider Bereiche ist durch einen robusten Ziegelrand akzentuiert, die als Aufkantung zur Aufnahme der Begrünung fungiert. Durch eine Überlappung beider Oberflächen - der des Parks und der des Stadtraumes - entsteht eine räumlich wirksame und ortspezifische Geste, die den Entwurf bestimmt.

Die konstruktive Entwurfsabsicht ist wie folgt formuliert: „Das neuartige an diesem Projekt ist die Verwendung eines Ziegelgewölbes, um halbvergrabene Räume in einem öffentlichen Park zu integrieren, wodurch eine Kontinuität der Schnittstellen und elegante Übergänge zwischen der grünen und der gepflasterten 
Oberfläche geschaffen werden. Gleichzeitig wird die Sicherheit der Nutzer gewährleistet sowie neue Details entwickelt, die dafür benötigt werden“. ${ }^{\text {I3 }}$

Die Morphologie der Schale wurde mit Hilfe von RhinoVAULT entwickelt, einem benutzerfreundlichen und intuitiven Programmbaustein für den materialgerechten Entwurf regel- und unregelmäßig gewölbter Formverläufe druckbeanspruchter Konstruktionen innerhalb der Modellierungsumgebung von Rhinoce$\operatorname{ros} 3$ D. ${ }^{\mathrm{I}}$

Die digitale Modellierung geschieht im frühen, kostengünstigen und flexiblen Bereich des Planungsprozesses und benötigt keine kostspielige Computerhardware. Die Bedienung erfordert architektonisches und strukturelles Grundwissen über Schalentypologien, jedoch keine spezifischen Programmierkenntnisse. Im Gegensatz zu üblichen Herstellungsmethoden digitaler Konstruktionen findet nach der Formfindung jedoch keine Verlegung der Ziegel mithilfe einer CAD/CAM-gesteuerten, teureren Fertigungstechnologie statt, sondern der Entwurf wird ausgeführt als manuell verlegter Ziegelverband im katalanischen Stil. Diese Technik fand im Mittelmeerraum und angrenzenden Regionen Anwendung und wurde ob ihrer Fähigkeit zur schnellen und wirtschaftlichen Errichtung von Gewölben, Treppen und anderen druckbeanspruchten Bauteilen geschätzt. ${ }^{15}$

Die Besonderheit des Konstruktionsverfahrens liegt unter anderem in seiner (Abb. 3) lediglich skelettartigen Schalung, die als räumliche Struktur für die Flächenverteilung aufgebaut wird. Die Absenz von materialintensiven Schalungskonstruktionen, wie sie gewöhnlich für Schalenbauwerke benötigt werden, verstärkt zusätzlich die wirtschaftlichen und prozessoralen Vorteile des Bauablaufes. Die Verlegung wird wie folgt beschrieben:

„,Für Fliesengewölbe wird ein leichtes Sandwich aus typischerweise drei Schichten dünner ( I 5 bis $25 \mathrm{~mm}$ ) Strukturfliesen verwendet, um den druckbelasteten Raum effizient zu überbrücken. Der schnellbindende (5-20 Sekunden, abhängig von den Umgebungsbedingungen) Gipsmörtel (Pariser Putz), mit dem die erste Fliesenschicht verlegt wird, reduziert den Bedarf an Schalungen, da das Mauerwerk temporär nicht abgestützt werden muss (in der Regel nur wenige freitragende Reihen, abhängig vom gewählten Kachelmuster). Diese erste 

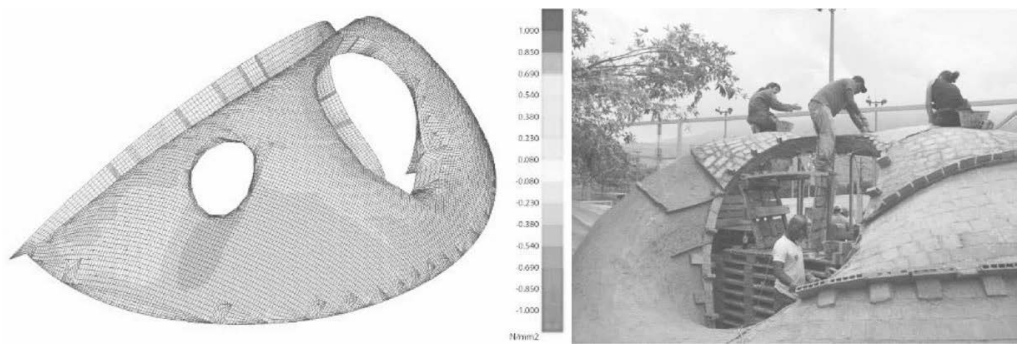

3 Links: Spannungsanalyse des Ziegelmodells; rechts: Bau des Gewölbes beim Übergang von der zweiten zur dritten Ziegelschicht zeigt, Block Research Group ETH Zürich 2014

Fliesenlage wird dann zu einer dauerhaften Schalung für den schnellen Aufbau der anderen Fliesenlagen, für die ein normaler Zementmörtel verwendet wird." ${ }^{16}$

Die Wahl dieser - eigentlich ortsfremden - Herstellungsmethode erscheint in diesem Zusammenhang trotzdem angemessen, da Kolumbien bereits über eine Tradition ornamentaler Ziegelarchitektur verfügt, ${ }^{17}$ das Material also bekannt ist und handwerkliche Kenntnisse für die Verlegung vorhanden waren. Die Entscheidung für den Herstellungsprozess hat Auswirkungen auf die Fertigungsgenauigkeit und Dimension des Gebäudes, aber auch für die notwendige Kompetenzstufe des ortsansässigen Konstruktionsteams, das eine individuelle Schulung für die Verlegung katalanischer Ziegelsteinverbände erhalten sollte. Frühere Projekte der Block Research Group in Äthiopien wurden in ähnlicher Weise ausgeführt und lieferten pädagogische Erfahrungswerte für die effiziente Schulung der Maurer-Neulinge in der Bauphase.

Diese „Hybridisierung“ der Prozesskette vom digitalen Modell zum handwerklichen Konstrukt ist von großer Bedeutung, erlaubt sie doch eine Anwendung avancierten architektonischen Fachwissens in Gesellschaften mit prekären wirtschaftlichen und technologischen Ausgangsbedingungen, denen der Zugriff auf CAMTechnologie üblicherweise erschwert ist. Diese kombinierte Entwurfs- und Konstruktionsmethodik erzeugt einen eigenen ästhetischen Ausdruck, der zwischen Tradition und Progressivität formal zu vermitteln vermag. 
Projekt Concrete[I]land Concrete[I]land ist ein filmisches Architekturprojekt, das im Jahre 2015 von der französischen Architektengruppe New Territories zusammen mit Studierenden im illegalen Slumgebiet Makkasan in Bangkok realisiert wurde. Projekttitel und einzelne narrative Versatzstücke sind inspiriert von James Graham Ballards Roman Betoninsel aus dem Jahre I974.

Zwei Kurzfilme geben fragmentarische Hinweise zum Konstruktionsprozess und dem sozialen Kontext einer funktional nicht näher bestimmten überdachten räumlichen Struktur. In der dystopischen Szenerie des ersten Films Concrete[I]Land- fictionshort movie (Länge 8:32 Minuten) (new-territories / R\&Sie(n) 20I5) führt ein ortskundiger, westlicher Führer den Betrachter in das Innere einer dichten, verwirrenden und beengenden SlumSiedlung, die von psychisch kranken Menschen bevölkert wird; Andeutungen von Drogenkonsum und sexueller Aktivität tragen zur verstörenden Atmosphäre bei. Der eigentliche architektonische Beitrag erscheint fast beiläufig zum Ende des Films, gezeigt wird dort ein organisch geformter Unterstand mit einer metallischgrauen Fassade aus additiv hergestellten Schindeln. Der Film legt nahe, dass das Baumaterial der Schindeln aus dem Granulat lokaler Abfälle gewonnen wird.

Im zweiten Film Sound - voice for RSI (real sensor interface) perturbations of trajectories/stochastic (Länge 0:5 I Minuten) wird das Herstellungsverfahren dieser Bauelemente genauer erklärt. Die futuristisch anmutende Szenerie, gefilmt mit einer fixen Kameraperspektive, zeigt einen am Tisch sitzenden, jungen Asiaten neben einem Roboterarm. Während dieser über eine Düse den Betonschlamm für die Schindelproduktion auf eine Wellblechform aufträgt, gibt der junge Mann einen monotonen Klang von sich. Der Film suggeriert, dass der Bewegungsablauf des Roboterarms durch die menschliche Stimme beeinflusst wird und diese akustische Information über eine veränderte Bewegungstrajektorie der Düse in die Form der Schindeln übertragen wird. Die einzelne Schindel fungiert nun als Archiv akustischer Informationen, die in der Morphologie gespeichert werden (Abb. 4): „Die Komponenten des Unterstandes werden mithilfe eines Robotersystems mit realem Sensor-Interface hergestellt... Dabei beeinflusst Nick [...] die Bewegungsabläufe der Düse. [...] Der Unterstand als versteinerte Stimme $[\ldots]^{*} .{ }^{18}$ 


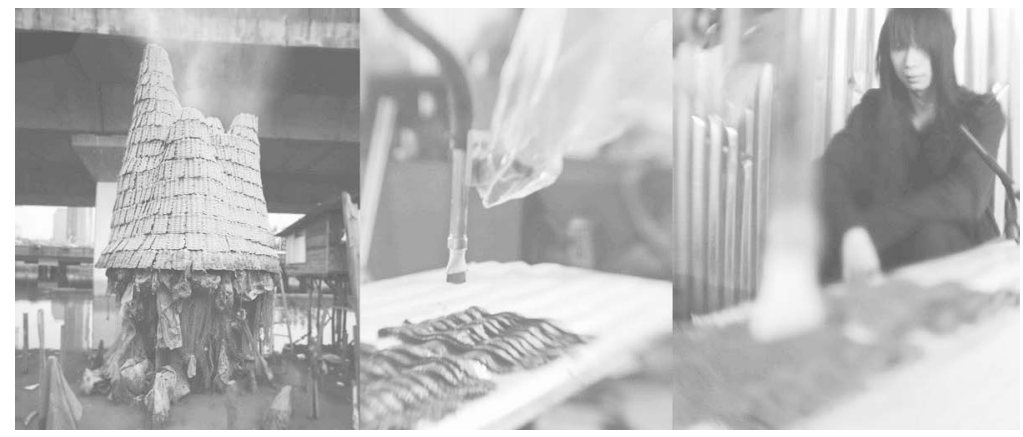

4 Links: Unterstand aus additiv gefertigten Schindeln, hergestellt aus recyceltem Material; mitte: Robotischer Verteilungsprozess; rechts: Akustische Anpassung der Roboterbewegung, NewTerritories, M4, with Innsbruck University, François Roche with Camille Lacadee and Stephan Henrich

Diese fragmentierte und surreale filmische Narration zeigt ein rätselhaftes, vage zukünftiges Universum, in deren Zentrum eine beunruhigende Ökologie menschlichen Verhaltens im ritualisierten Austausch mit computergestützter Technologie steht. Die menschlichen Laute, die den Bewegungspfad der Maschine bestimmen, folgen keiner erkennbaren Melodie, sie zeigen auch keinerlei semantische Strukturen, sondern erinnern vage an schamanistische Gesänge.

Die kultische Interaktion von Mensch und Maschine führt in diesem Projekt zu einer technologisch ermöglichten, spirituellen Aufladung des Bauwerks jenseits der reinen Materialität, sie verhandelt unumkehrbare materielle Objekthaftigkeit und menschliches Handeln.

Projekt Multisensory Architecture Multisensory Architecture untersucht die Rolle der Architektur als therapeutisches Instrument für Kinder mit Autismus-Spektrum-Störung (ASS). Diese Störung beeinflusst die Kontrollfähigkeit der Patienten über eingehende Impulse multimodaler Sinnesdaten (die normalerweise durch neurologische Filterung erzielt werden) und deren anschließende Umsetzung in aufeinander abgestimmte verhaltensmäßige und motorische Handlungen (Abb. 5). 


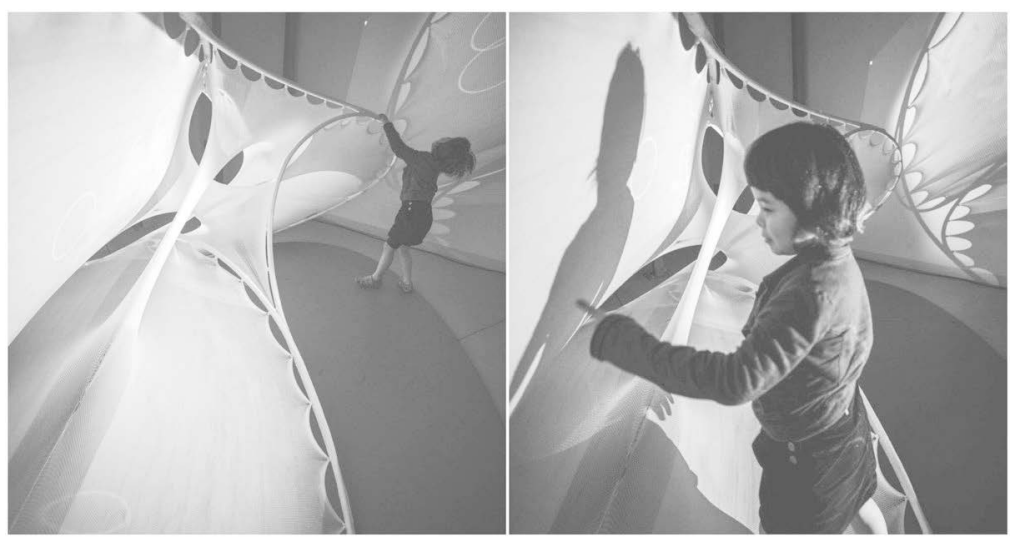

5 Räumliche Geometrie der sensorischen Spiellandschaft mit erweiterten visuellen und akustischen Rückkopplungsfunktionen, Sean Ahlquist 2017

Der Entwurfsansatz der transdisziplinären Forschungsgruppe aus Architekten, Psychiatern und Kinesiologen orientiert sich an einem nicht-reduktionistischen, dynamischen und systemischen Verständnis menschlichen Handelns. ASS wird unter dieser Prämisse als Verbund singulärer sensorischer Behinderungen verstanden, die sich als Beeinträchtigung der motorischen oder kommunikativen (verbalen und nonverbalen) Fähigkeiten der Patienten manifestieren. Therapeutische Maßnahmen zur Behandlung von ASS wurden aus diesem Grunde als multi-sensorische Patienteninteraktion konzipiert. Die Forschungsgruppe entwickelt einen Entwurf für eine kindgerechte ,multivalente Architektur von taktilen Interfaces, physischen Landschaften und Instrumenten der sozialen Interaktion"I9 aus einer elastischen textilen Oberfläche, die durch gebogene GFK-Randstäbe ausgesteift wird. Eine interaktive Projektion mit einstellbaren akustischen und visuellen Informationen erlaubt kalibrierbare therapeutische Maßnahmen zur Steigerung der räumlichen und sensorischen Empfindlichkeit der Patienten: „Die Oberfläche des Textils wird durch eine Reihe von Triggern bestimmt, die von einer Microsoft Kinect erfasst werden, um festzustellen, wann das Textil an einer solchen Stelle bis zu einer bestimmten Tiefe gedrückt wurde. [...] Sobald ein Trigger gefunden wurde, werden die folgenden Schritte eingeleitet: (I) Ein Kreis pulsiert, um die Erinnerung daran zu stärken, wo sich der 
Aktivierungspunkt befindet. (2) gefolgt von der Projektion einer Animation über die Oberfläche des Textils und (3) Abspielen eines mit dem Inhalt der Animation synchronisierten Soundclips."20

Die Knotenform der Flächen bildet den Potenzialraum motorischer Bewegungsmuster eines im Zentrum interagierenden Menschen, andere spielerische Interaktionen sind (Abb. 6) ebenfalls vorstellbar. Die Aktionspotenziale der therapeutischen Oberfläche werden einzeln aktiviert und zusammengestellt, jede Patienteninteraktion löst in der Folge eine individuelle Kombination visueller, akustischer und räumlicher Impulse aus.

Die hybride Struktur erlaubt darüber hinaus die Überwachung und Analyse der Bewegungsmuster des Kindes mithilfe der verschiedenen Interaktions-Schnittstellen. Die Koordination dieser Funktionen innerhalb eines einzelnen Objekts erfordert eine interund transdisziplinäre Arbeitsmethodik zwischen Psychologie, Kinesiologie, Textildesign, Interaktionsdesign und Architektur. Das Wissen computergestützter Gestaltung findet so eine therapeutische Anwendung komplexer, dynamischer Prozesse, die räumlich und interdisziplinär realisiert werden können und sich in der materiellen Form der Struktur manifestieren.

Schlussbemerkung Die drei hier vorgestellten Architekturprojekte stellen unterschiedliche Beziehungen zwischen computerbasierten Prozessen und anderen Themenfeldern her. Diverse Medien operieren dabei als individualisierte und transformative Schnitt-

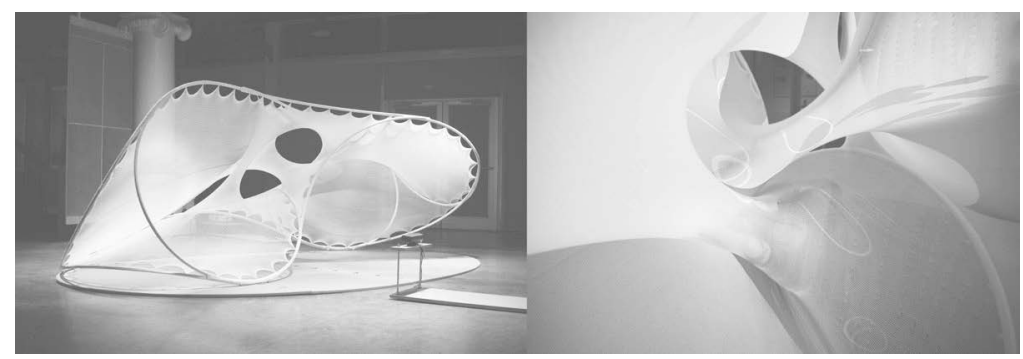

6 Links: Therapeutische interaktive Oberfläche aus CNCgestrickten, nahtlosen, elastischem Textil und aktiv gebogenen GFK-Stäben, rechts: Spielendes Kind in Interaktion mit textiler Oberfläche und Projektionen, Sean Ahlquist 2017 
stellen zwischen menschlichen und technologischen Akteuren, sie verfolgen einen experimentellen und kritischen Mediengebrauch, der einem technisch entwickelten eigenständigen Gestaltungsbegriff verpflichtet ist. Die Eigenständigkeit dieses Ausdrucks - in und aus der Zeit - entsteht aus einer Amalgamierung von Kulturtechniken, Wissenschaften und künstlerischen Positionen. Die Implikationen dieses Vorgehens sind auch für die Ausbildung von zukünftigen Architekten, Architektinnen und Gestaltern von Bedeutung, erfordern sie doch eine mediale Eloquenz und fachliche Expertise in vielen Wissensfeldern und ein konzeptionelles Verständnis für deren Übertragung in bestehende und noch kommende computergestützte Entwurfs- und Fabrikationsprozesse. Die in den Projekten erkennbare transdisziplinäre Methodik integriert hierbei selbstverständlich die Rückkopplungen der angewandten technischen Entwurfsmedien in ihrer Agency als konzeptionelle Elemente eines sich erweiternden Begriffsverständnisses architektonischer Formgebung und Raumbildung.

\section{Anmerkungen}

1 Eine Beschreibung dieser technologischen Neuordnungen am Beispiel der additiven Fertigung finden sich hier: Norbert Palz, Emerging Architectural Potentials of Tunable Materiality through Additive Fabrication Technologies. PhD Thesis, Centre for Information Technology and Architecture, Copenhagen 2012, S. 59.

2 Der britische Architekturtheoretiker Robin Evans hat die Wechselwirkung zwischen Planungswerkzeug, Geometrie und gestalterischem Vokabular ausführlich untersucht. Sie dazu Robin Evans, The Projective Cast Architecture and Its Three Geometries, Cambridge Mass. 1995.

Palz (Anm. 1), S. 118-122.

4 Hübsch ist sehr explizit in seinem Aushandlungsprozess zwischen typologischer Identität und struktureller Effizienz. Er schreibt in Heinrich Hübsch: Bau Werke, Karlsruhe 1838, S. 44: „Auch handelt es sich beim architektonischen Entwerfen immer um ein $A b$ - und Zu-Geben, um eine Verschmelzung der verschiedenen Formen, welche einerseits die Bestimmung des Gebäudes erheischt, anderseits die Statik verlangt. Deswegen kann die letztere Seite nie rein für sich berücksichtigt werden, sondern muss sich häufig nach der ersten modifizieren; so dass viele Proben nötig sind bis man die in doppelter Beziehung vorteilhafteste Formen gefunden hat". Die Aushandlung der beiden Kategorien - Struktur und Identität - war 
in seinem Fall allerdings nicht ausgewogen. Die Faszination für den propagierten Stil des Historismus überschattete das vor ihm liegende innovative Potenzial eines materialbewussten Entwerfens - abseits stilistischer Vorcodierungen. Hübsch verfügte also über das avancierte Instrumentarium zur räumlichen engen Korrelation von Form und Struktur, sah aber bewusst davon ab, diese als konzeptionelle Entwurfsidee konsequent weiterzuverfolgen. Siehe seine unglückliche Schlussfolgerung in Hübsch, ebd., S. 53.

5 Über die hegemonialen Implikationen von Technologie und Wissenschaft siehe auch Jürgen Habermas, Technik und Wissenschaft als „Ideologie". Berlin 1968, S. 52-54. Dieser potenziell disziplinierende Effekt, der über die inhaltsrelevanten technologischen Bedingungen zur Entfaltung kommen konnte, wurde schon früh diagnostiziert. Jean-François Lyotards Kommentar im Jahre 1979 folgt auch dieser Richtung. Er schreibt in The Postmodern Condition. A Report on Knowledge, Manchester 1979 [1984], S. 4: „Mit der Hegemonie der Computer geht eine bestimmte Logik und damit eine Reihe von Vorschriften einher, die bestimmen, welche Aussagen als ,Wissensaussagen' akzeptiert werden".

6 Im deutschen Raum lassen sich erste Versuche einer Computerkunst in der zweiten Hälfte der 1950er Jahre nachweisen. Diese Grafiken, verfolgten eine wissenschaftlichere Herangehensweise an die Künste und waren von der Theorie einer "Generative[n] Ästhetik", die von Max Bense vertreten wurde, beeinflusst. Diese Herangehensweise hatte ihre Ursprünge in einer Neukonzeption künstlerischen Arbeitens als postfaschistische Praxis.

7 Es soll allerdings hervorheben werden, dass Christopher Alexanders Antrieb für die Entwicklung der Mustersprache seinem Bedürfnis nach humanistischen am Individuum ausgerichteten Wohnbedingungen entstammte, rationale Optimierung und ökonomische Gewinnsteigerung waren nicht Teil der Agenda. Siehe dazu Christopher Alexander, "A City is Not a Tree", Architectural Forum, Vol 122. 1965, 1: S. 58-62.

8 Siehe auch Ingeborg Rocker, „Architecture of the Digital Realm. Experimentations by Peter Eisenman, Frank O. Gehry", 10. BauhausKolloquium Weimar, 2007, S. $249 f$.

9 Die Anwendung digitaler Entwurfsmedien als Instrumente einer neoliberalen Baupraxis wurde von Patrik Schumacher auf markante Weise gefördert. Für eine kritische Auseinandersetzung siehe die Publikation von Matthew Poole and Manuel Shvartzberg. The Politics of Parametricism. Digital Technologies in Architecture, London 2015. 
10 Christopher Alexander, "A Much Asked Question about Computers and Design", Speech at "Architecture and theComputer" Boston Architectural Centre, Boston, Dezember 1964.

11 Siehe Taubmann College "Social Sensory Surfaces" 2016: www.taubmancollege.umich.edu/research/research-through-making/2015/ social-sensory-surfaces [06.10.2019]

12 Weiteres Material zum Thema findet sich hier: www.block.arch. ethz.ch/brg/files/lopezlopez-2014-iass-thin-tile-vault-seventh-worldurban-forum-medellin-2014_1410359059.pdf [30.10.2019]

13 Siehe Block Research Group (Anm. 12).

14 Die ursprünglichen Anwendungsgebiete dieser Formfindungsmethode waren in der konservatorischen Analyse historischer Gebäudestrukturen beheimatet und wurden in der Folge zu einem benutzerfreundlichen und interaktiven Formfindungswerkzeug für den Architekturentwurf ausgebaut.

15 Die ersten Patente für diese Bauweise wurden ab 1885 von Rafael Gustavino angemeldet; eine Beschreibung des wesentlichen Prozesses finden sich hier: Raffael Gustavino, Building Tile, USA Patent 548, 160. 15 Oktober 1895.

16 Lara Davis, Mathias Rippmann, Tom Pawlofsk und Phillippe Block, „Innovative funicular tile vaulting. A prototype vault in Switzerland", The Structural Engineer, 2012, S. 46.

17 Dieser Prozess ist in großer Sensibilität für eine möglicherweise kolonialisierend wirkende Auslegung durchzuführen und in Respekt vor lokalen Traditionen zu gestalten.

18 Camille Lacadee, François Roche, Vongsawat Wongkijjalerd und Daniela Mitterberger, concrete[i]land. Edited by new-territories / R\&Sie(n), 2015: www.new-territories.com/blog/?p=2161 [30.10.2019].

19 Sean Ahlquist, „Sensory material architectures. Concepts and methodologies for spatial tectonics and tactile responsivity in knitted textile hybrid structures", International Journal of Architectural Computing, 2016, , S. 1.

20 Sean Ahlquist (Anm. 19), S. 14. 


\section{Hacking Agency: Digitale Fabrikation als Entwurfsmedium}

Die digitale Fabrikation verändert die Beziehung zwischen dem Entwerfen und der Herstellung in der Architekturpraxis. Der Diskurs um die Auswirkungen der digitalen Herstellungsverfahren auf Praktiken in der Architektur hebt einige positive Aspekte dieser Technologien hervor: Eine höhere Auflösung und genauere Detailierung, die Möglichkeit, Formen zu verwirklichen, die mit traditionellen Fertigungen schwerlich realisierbar wären, oder auch den nahtlosen Übergang von Entwurfsabsichten zu materiellen Artefakten. Anstatt die Technologien einfach als solche $\mathrm{zu}$ verwenden oder zu betrachten, schlägt dieser Beitrag das ,Hacking' als alternativen Ansatz vor. Auf der einen Seite entwickeln sich damit neue Verläufe von Entwurfsuntersuchungen, indem digitale Herstellungsverfahren über ihre vorgesehene Nutzung hinausgetrieben werden, auf der anderen Seite offenbart dies die inhärenten Anforderungen und Tendenzen gegenüber diesen Technologien. Beim Hacking geht es um mehr als um ein bloßes Aufzwingen von Ideen auf ein fabriziertes Artefakt. Es geht einher mit dem Erkennen einer Agency der Technologien selbst und eröffnet einen neuen Umgang mit ihnen. Basierend auf dem Projekt Encoded Matter wird in diesem Beitrag argumentiert, dass digitale Herstellungsverfahren keine neutralen und transparenten Werkzeuge sind, sondern Entwurfsmedien; somit wird das Entwurfsergebnis durch die Materialien, die besonderen Eigenschaften der Produktionsmaschinen und den alles steuernden Code beeinflusst. Mit anderen Worten: Material, Maschine und Code haben allesamt Einfluss auf den Entwurfsprozess, bedingt durch computation und digitale Fabrikation. 
Entwerfen, Herstellen und Hacken Die Aktivitäten des Entwerfens und Herstellens von Architektur geschehen tendenziell in einem räumlichen und zeitlichen Abstand. Diese Trennung weist eine lange Geschichte auf, die auf die Entstehung der Architektur als Beruf und dessen Emanzipation von der Konstruktionspraxis hin zu einer intellektuellen freien Kunst zurückgeht. ${ }^{I}$ Architekten und Architektinnen produzieren keine Architektur, stattdessen betreiben sie die Herstellung von Architektur durch intervenierende Medien $^{2}$, indem sie zum Beispiel Skizzen, Zeichnungen, Modelle oder Beschreibungen anfertigen. Im Laufe der Jahrhunderte wurden der architektonische Beruf und die Praxis durch Medien bestimmt, die von Architekten genutzt werden, wobei die Entwicklungen von Technologie, Wissenschaft und Kultur mit einflossen. Während manche Entwurfsmedien entscheidend die Architekturproduktion beeinflussen, sind andere Medien spekulative Mittel, die es dem Architekten oder der Architektin erlauben, eher abstrakte Raumkonzepte zu untersuchen und architektonische Projekte zu entwickeln, die jenseits des Reichs des Bauens liegen.

Dennoch bleibt der Bereich zwischen Entwerfen und Herstellen, die körperliche Beschäftigung mit Materialität und das Bilden von Architektur ein bedeutender Aspekt der Architekturpraxis. Architekten und Architektinnen beschäftigen sich mit Materialität und dem Herstellen anhand von Materialtests, maßstäblichen Modellen und Prototypen; auch Grundstücksbesichtigungen bleiben unverzichtbar. Entwurfsmedien können als Brückenschlag zwischen Entwerfen und Herstellen, zwischen dem Abstrakten und dem Körperlichen ${ }^{3}$ verstanden werden - und dies in beide Richtungen: Sie sind nicht nur Hilfsmittel, um Entwurfsideen zu verwirklichen oder zu konstruieren, sie werfen auch Fragen über die Herstellung und Materialität für das Verständnis der Architekten und Architektinnen auf.

Die Auswirkungen digitaler Technologien auf die Architekturpraxis kann in dieser Tradition von Entwurfsmedien und der Übernahme von Technologien verstanden werden. Die ersten Jahrzehnte der Anwendung digitaler Technologien haben vor allem die Medien zum Entwerfen beeinflusst. Frühe computergestützte Entwurfswerkzeuge wurden als digitale Versionen von altbekannten, analogen Praktiken des Skizzierens, Modellierens oder Darstellens betrachtet. Architekten und Architektinnen nutzten diese digitalen Werkzeuge oder übernahmen sie von anderen Disziplinen, 
um solche Architekturen zu entwerfen, die mit analogen Mitteln undenkbar gewesen wären. ${ }^{4}$ Doch sie blieben im Bereich der Bilder oder wurden durch konventionelle Zeichnungen in gebaute Artefakte übersetzt. Jüngere Entwicklungen in der digitalen Fabrikation beeinflussen direkt, wie Artefakte materialisiert oder wie Informationen im Verhältnis von Entwurf und Herstellung übersetzt werden. Der Diskurs über digitale Fabrikation in der Architektur betont einige positive Auswirkungen hinsichtlich der Verwirklichung von Formen, die mit traditionellen Herstellungsverfahren und Methoden nicht realisierbar gewesen wären, dem zunehmend genaueren Auflösungs- und Detailierungsgrad sowie der Ermöglichung von Massenanpassungen jenseits der Standardisierung. Digitale Fabrikation ist eine Triebkraft für Konvergenz, egal ob es die Schließung der Lücke zwischen ganz verschiedenen Disziplinen ${ }^{5}$ bedeutet oder die Auflösung der Lücke zwischen Entwurf und Herstellung ${ }^{6}{ }^{6}$ so ergibt sich ein problemloser und ununterbrochener Übergang von Entwurfsideen zu verwirklichten Artefakten.

Dieser Beitrag betrachtet digitale Fabrikation weder als eine neutrale Technologie noch als Potenzial, die Lücke zwischen Entwurf und Herstellung zu schließen. Vielmehr regt er dazu an, digitale Fabrikation als ein Medium zu betrachten, das zur Entstehung von Entwurfsideen beiträgt. Die Untersuchung prüft, wie sich Eigenschaften von Materialien und Maschinen in Entwurfsmodellen im Rahmen digitaler Fabrikation und anderer Mittel zur Kodierung entwickeln oder wie sie sich selbst in materiellen Artefakten zeigen. Digitale Fabrikation wird als eine Reihe von Technologien betrachtet, die mit ganz bestimmten Verknüpfungen von Entwurf und Herstellung sowie von abstrakten Codes und der Materialität hergestellter Artefakte umgehen. Anstatt Technologien so zu nutzen, wie sie eigentlich gedacht sind, regt dieser Beitrag dazu an, Hacking als alternativen Ansatz zu betrachten, mit dem Grundzüge der Technologien digitaler Fabrikation enthüllt werden können. Das Hacking geht über ein bloßes Aufzwingen von Ideen auf ein fabriziertes Artefakt hinaus, indem die Agency dieser Technologien erkannt und überwunden wird.

Forschungsprojekt und Kontext Die Argumentation dieses Beitrags basiert auf dem Forschungsprojekt Encoded Matter, das an dem MMlab, Architekturfakultät der KU Leuven7 durchgeführt wurde. Das MMlab ist ein Forschungs- und Fabrikationslabor, das 
die Rolle von Entwurfsmedien in der Architektur durch projektbasierte, interaktive Experimente hinterfragt. Das Projekt ist Teil meiner PhD-Forschung mit dem Titel Negotiating Agency. Computation \& Digital Fabrication as Design Media. ${ }^{8}$ Diese untersucht die Agency von Material, Code und Maschine in architektonischen Entwurfsprozessen mit Hilfe von digitalen Technologien basierend auf Fallstudien aus der Entwurfspraxis und -lehre.

Das Projekt Encoded Matter besteht aus einer Serie von Entwurfsexperimenten, die das Potenzial sowie die Grenzen von kostengünstigen und selbstgebauten Open-Source-Produkten als spezifische Fabrikationstechnologien ergründen. Mit dem Projekt soll aufgedeckt werden, wie die Materialien, die Fabrikationsmaschinen und der Code, der diese steuert, die Materialität der fabrizierten Artefakte beeinflussen. Für das Projekt war von Bedeutung, dass die Technologie zugänglich, offen und ,hackbar“ war, sowohl bezüglich der Hardware als auch der Software.

Dies offenbarte bei näherer Betrachtung, wie die Fabrikationsprozesse Spuren hinterließen oder sich mit der Zeit entwickelten und wie dieser Vorgang gehackt werden könnte, um bestimmte Materialeigenschaften in den fabrizierten Artefakten zu bewirken.

Spuren und Fäden Materialien durchlaufen mehrere Transformationen bevor sie in der Architektur verwendet werden: Prozesse des Wachstums, der Sedimentation, der Synthese. Rohmaterial wird entnommen und $\mathrm{zu}$ Materialien, Bauteilen und Produkten für das Bauen weiterverarbeitet. Materialinformationen setzen sich während des Konstruierens fort, genau wie später durch die Nutzung, Alterung, Verwitterung oder durch Reparaturen. All diese Prozesse hinterlassen Spuren an den Materialien und den daraus konstruierten Artefakten. Das bewusste Entwerfen mit solchen Spuren weist eine lange Geschichte in der Architektur auf. ${ }^{9}$

Die Spuren, die wir in entworfenen Artefakten vorfinden, sind nicht nur Resultat der oben beschriebenen Prozesse, sondern auch Spuren von Materialanpassungen durch industrielle Prozesse in Form von Blechen, Trägern oder sonstigen Bauteilen. Tim Ingold argumentiert, dass die Vorstellung von Architekturen, die aus geometrischen Komponenten wie riesige Puzzles zusammengebaut sind, relativ neu ist. Er verortet die Wurzeln der Architektur in Praktiken des Webens, Korbflechtens, der Schreinerei und weiterer Handwerke. In diesen Praktiken wird Form nicht als Baukasten- 
prinzip vorgegeben, sondern resultiert aus dem allmählichen Prozess des Webens von Fäden oder dem allmählichen Formen von Materialien aufbauend auf einem Verständnis für ihren bestimmten Aufbau, wie in der Stein- oder Holzbearbeitung. ${ }^{\text {Io }}$

Die Verwendung von Materialien in der digitalen Fabrikation kann mithilfe der oben aufgeführten Ideen verstanden werden: In der digitalen Fabrikation verwendete Materialien neigen dazu, in hohem Maße standardisiert und industriell ${ }^{\mathrm{II}} \mathrm{zu}$ sein - unter anderem Bleche sowie massive Materialien zum Schneiden oder Fräsen, Filament oder Pulver für zusätzliche Fertigungen - doch gefertigte Artefakte neigen dazu, äußerst spezifisch, nicht standardisiert und einmalig zu sein. Das hat somit zu noch komplexeren Teilen des Baukastens geführt, indem jeder Bestandteil einer Struktur einmalig ist und geometrisch beschrieben sowie exakt gefertigt werden kann. Prozesse digitaler Fabrikation hinterlassen ihre eigenen Spuren - Spuren durch Sägeblätter, verbrannte Kanten beim Laserschneiden oder den geschichteten Aufbau additiver Fertigung. Anstatt digitale Fabrikation für Präzision oder exakte Kopien digitaler Modelle zu verwenden, schlägt Encoded Matter anhand von Untersuchungen der Spuren einen alternativen Umgang mit solchen Fabrikationstechnologien vor. Für einen solchen Ansatz ist die Offenheit der Technologie entscheidend, um digitale Fabrikation als einen Prozess verstehen zu können, der sich mit der Zeit entfaltet.

Offene Technologie Das Projekt Encoded Matter hat von der zunehmenden Zugänglichkeit und Demokratisierung digitaler Fabrikation in der Architektur profitiert. Für die in diesem Beitrag erläuterte Forschung waren zwei Projekte besonders einflussreich: Das FabLab-Projekt, das von Neil Gershenfeld ${ }^{12}$ am MIT initiiert wurde, zielte darauf ab, Herstellungstechnologien für Studierende, Designer und ein breites Publikum zugänglich zu machen. Dies führte zu einer größeren Beteiligung und resultierte in einem globalen Netzwerk aus FabLabs. Zum anderen war das RepRapProjekt von großer Bedeutung, das von Adrian Boyer im Jahre 2005 an der University of Bath initiiert wurde. Der Projektname steht für „Replicating Rapid Prototypes“, ein Open-Source-3D-Drucker, der in der Lage ist, die meisten seiner eigenen Teile zu produzieren. Seitdem entstanden viele weitere preisgünstige $3 \mathrm{D}$-Drucker mit Open-Source Verfahren zum selber bauen. ${ }^{13}$ 
Das MMlab wurde im Geiste des FabLab-Projekts gegründet und strebt an, den Studierenden und der Fakultät der Sint-Lucas School of Architecture digitale Fabrikationstechnologien näherzubringen. In diesem Zusammenhang habe ich 2009 eine RepRapMaschine gebaut, die mir ein gutes Verständnis für einige der Schwierigkeiten sowie Potenziale der Technologie ermöglicht hat (Abb. I). Das Projekt Encoded Matter hat von der Zugänglichkeit, Offenheit und den geringen Kosten für die Technologie profitiert. Beim Drucken habe ich mit einer Maschine auf meinem Schreibtisch gearbeitet, mit welcher der Prozess in Echtzeit erlebt werden konnte, ohne Angst zu haben, im möglichen Durcheinander Dinge zu zerbrechen. Dies war ein befreiendes Gefühl im Gegensatz zu dem Umgang mit geschlossenen Technologien, wie zum Beispiel mit teuren Maschinen, die in anderen Entwurfsuntersuchungen aus der Ferne über Online-Schnittstellen ${ }^{\mathrm{I} 4}$ verwendet werden.

Der Drucker verwendete ein Verfahren, das „Fused Filament Fabrication"I5 genannt wird: Ein thermoplastisches Filament wurde einem Extruderwerkzeugkopf zugeführt, in dem der Kunststoff geschmolzen wurde. Der Werkzeugkopf bewegte sich in $\mathrm{x}$ und y-Richtung, gab Material ab und bildete eine Schicht; dann wurde die Plattform in der z-Richtung abgesenkt, woraufhin die nächste Schicht abgegeben wurde. Die Drucke wurden Faden für Faden und Schicht für Schicht aufgebaut, was in der typischen linienbasierten und geschichteten Materialität resultierte. Die Technologie wies einige Einschränkungen und Schwierigkeiten auf. Jede Schicht musste zum Teil von der vorigen Schicht unterstützt werden, auch wenn Überhänge von bis zu 45 Grad möglich waren. Es gab einen Temperaturunterschied zwischen den bereits aufgebrachten Schichten und denen, die gerade erst gedruckt wurden. Weil das Material beim Abkühlen schrumpfte, kam es zu Verzerrungen des Drucks. Wenn sich der Werkzeugkopf über eine Lücke in dem Druck bewegte, dann wurde die Extrusion gestoppt, doch etwas Material tropfte aus der Düse nach, sodass sich an der Seite des Drucks Fäden bildeten. Der Übergang von einer digitalen Datei zu einem materiellen Druck basierte auf folgenden Schritten: Es wurde ein Entwurf modelliert, der durch eine digitale 3D-Datei generiert wurde; dieses Modell wurde mit Hilfe einer externen Software verarbeitet und die daraus resultierende Datei wurde an die Maschine gesendet und gedruckt. 


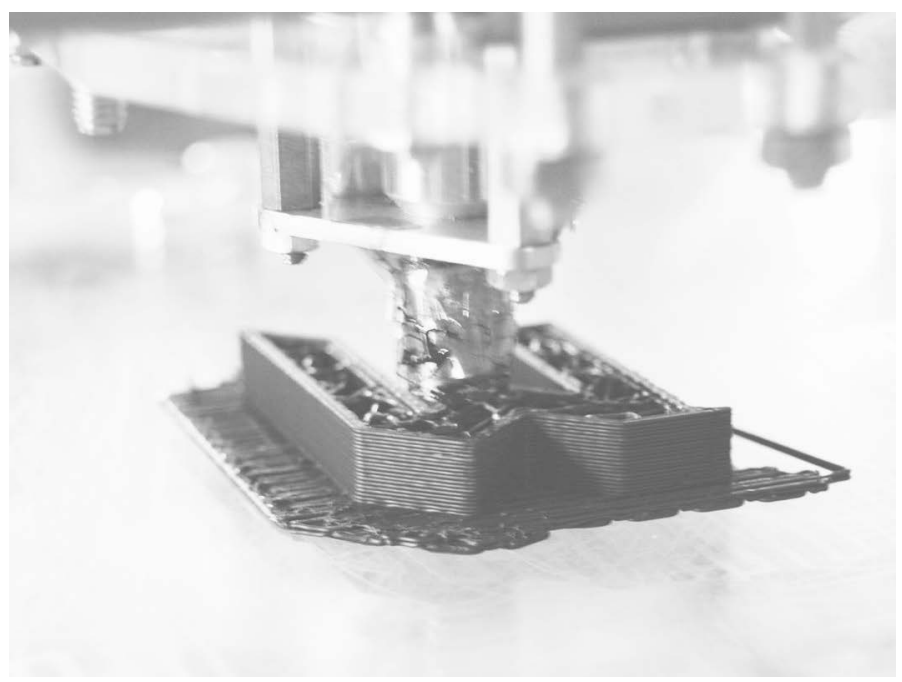

Fabrikation in Raum und Zeit Das Entwerfen durch das Schreiben eines generativen Codes ist ein grundsätzlich reversibler und dynamischer Prozess, wobei Entwurfslösungen aus einem Zusammenspiel von Designer, Code und entstandenem Produkt resultieren. Damit das Ergebnis materialisiert werden kann, muss dieser dynamische Prozess angehalten und statische Dateien müssen exportiert, umgewandelt und zur Fertigung an die Maschine gesendet werden. Artefakte, die durch das Schreiben generativer Codes entworfen werden und mit der Verwendung digitaler Fabrikationsmaschinen produziert werden, sind ebenso das Ergebnis der diskreten und reversiblen Zeit des Codes wie auch des unumkehrbaren und unterbrochenen Einfrierens der Zeit von statischen Dateien und der kontinuierlichen, aber unumkehrbaren Zeit des Materialisierens und Herstellens.

Encoded Matter begann mit einem Interesse für dieses Einfrieren der Zeit und die Transformationen zwischen den dynamischen, reversiblen Prozessen der Verwendung generativer Codes, den Prozessen der Materialisierung und dem resultierenden, statischen sowie materiellen Artefakt. Beim genauen Ablesen des Prozesses zeigt sich eine Entwurfsumgebung mit verschiedenen Ebenen kontinuierlichen Feedbacks, in der sich der Entwurf durch bestimmte diskrete, irreversible Schritte entwickelte. Entwürfe 


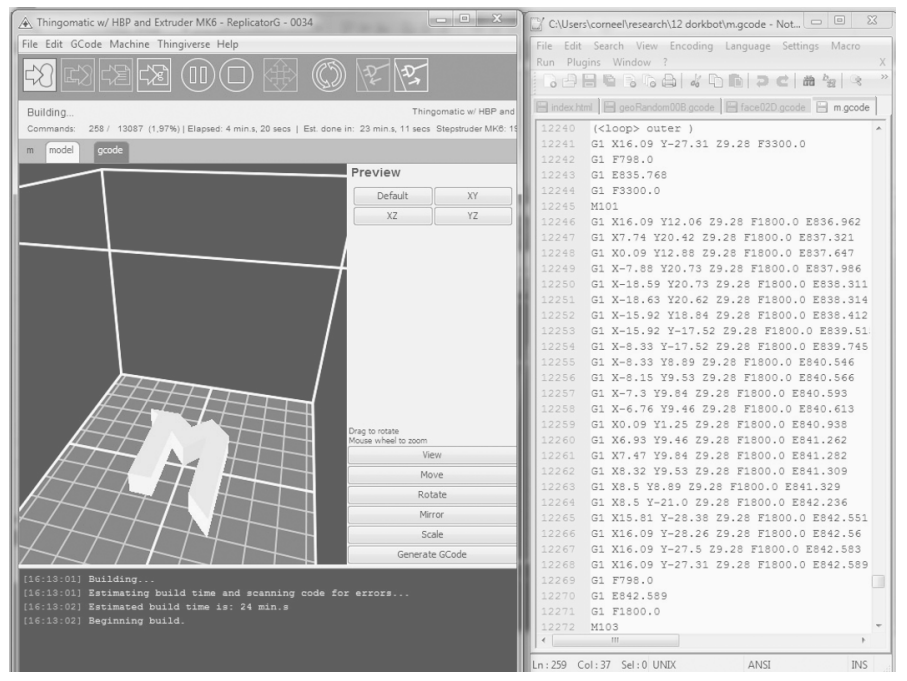

2 ReplicatorG slicer Software

werden durch Codes generiert und anhand von Bildern am Bildschirm ausgewertet. Der resultierende Entwurf könnte entweder durch die Manipulation von Parametern indirekt beeinflusst werden oder direkt durch Anhalten der Software, die Veränderung des Algorithmus und das Neustarten der Software. Sobald dadurch ein interessanter Moment oder eine Variation erreicht wurde, musste eine statische Datei exportiert werden. Dieses ,Mesh file ' wurde dann von einer externen Software aufgenommen und von dieser geschnitten, woraufhin eine, $g$-Code -Datei vorbereitet wurde, die dann an den Drucker gesendet wurde (Abb. 2). Während die Bilder auf dem Bildschirm nur ein bestimmtes, eingeschränktes Feedback boten, war es meist nur durch die konkrete Herstellung möglich, die Ergebnisse zu bewerten.

In dem Prozess der Erstellung eines 3D-Drucks waren die ,Mesh-' und ,g-Code'-Dateien statische und irreversible Stadien, aber sie erlaubten eine alternative Kontrolle über den Herstellungsprozess und wie er sich mit der Zeit entfaltete. Während das ,Mesh' die äußere Form des Artefakts lediglich als annähernd dreieckig erfasste, codierte die ,g-Code -Datei mit der Zeit den Prozess seiner Herstellung. Eine ,g-Code -Datei enthielt verschiedene Arten von Befehlen: m-Codes, die maschinenspezifisch sind und zum Beispiel die Temperatur des Heizbetts und des Extruders fest- 


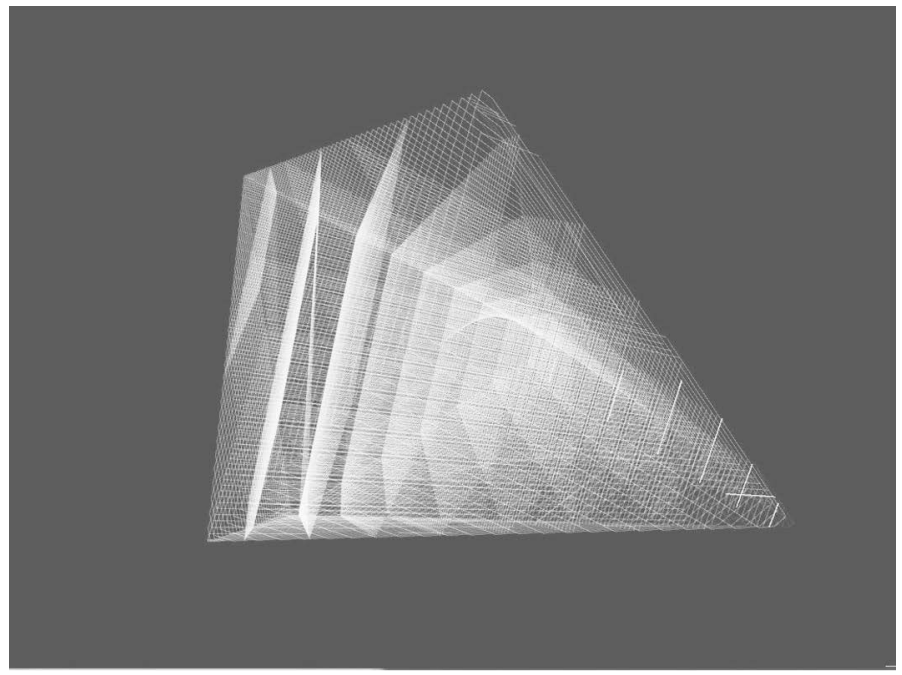

3 Benutzerdefiniertes Entwurfswerkzeug

legten; g-Codes, welche die Position des Extruders kontrollierten; F-Codes, welche die Geschwindigkeit kontrollierten und E-Codes, welche die Menge an Material kontrollierten, das ausgestoßen wird. Zusammen bieten sie Kontrolle über die Menge an hinterlegtem Material in Raum und Zeit. ${ }^{\mathrm{I}}$

Entwurfsuntersuchung Der Ausgangspunkt des Projekts Encoded Matter war es, über die volumetrische Beschreibung des Artefakts als ,Mesh ' hinauszugehen und den g-Code direkt aus einem benutzerdefinierten Entwurfswerkzeug zu generieren, das während der Verarbeitung unter Anwendung der externen Code-Thread-Bibliothek $^{17}$ geschrieben wurde. Während der Entwurfsuntersuchung wurden verschiedene Serien von Artefakten entworfen und hergestellt und so wurde je ein bestimmtes Entwurfswerkzeug für jede der Serien entwickelt. Nach einer Serie von Tests, mit denen das Konzept überprüft wurde und spielerisch verschiedene Parameter verstanden und angepasst wurden, wurde eine Serie von Materialexperimenten in der unten beschriebenen Reihenfolge ausgeführt (Abb. 3).

Serie 1: Objekte ohne Haut In der ersten Serie (Abb. 4-5) wollte ich mit den Objekten die Idee des Überspringens des, Mesh' als 


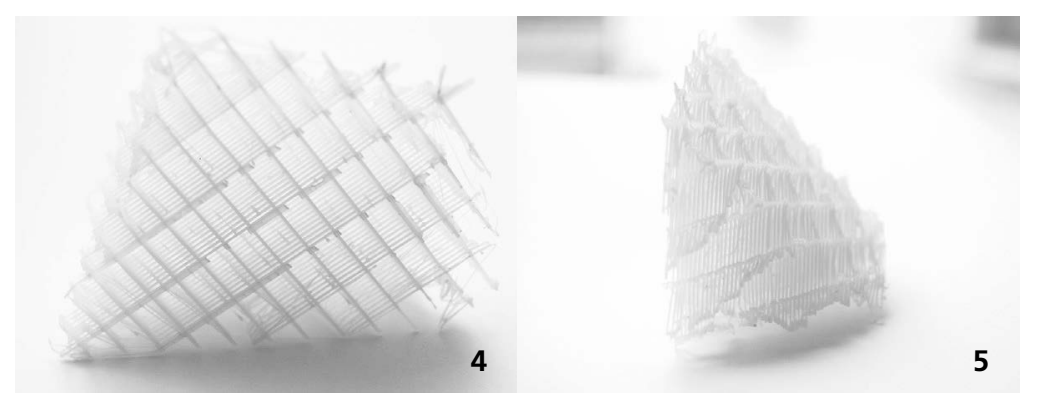

4-5 Objekte ohne Haut

Zwischenschritt ausdrücken sowie den inneren Materialaufbau des Artefakts zeigen und habe mich dafür entschieden, mehrere Objekte ohne eine Haut zu drucken. Die innere Struktur war als dreieckiges Raster entworfen, das in zwei Richtungen gedreht werden kann. Auch wenn es nicht explizit im g-Code selbst bestimmt wurde, wurde das äußere Volumen der Objekte von verschieden angewinkelten Ebenen zerschnitten. Somit haben sie sich mit den rotierten Rastern in unterschiedlichen Winkeln überschnitten, wodurch jeder Fläche des Artefakts eine andere Textur und somit ein anderer Materialausdruck verliehen wurde. Die Struktur des Rasters der ersten Serie wurde von einem Algorithmus kontrolliert; dessen Struktur war identisch mit üblichen, Fill-Strukturen', die in , slicing software" zu finden sind. Es wurden verschiedene Drehungen und Abstände in dem Raster getestet, sodass das Raster in allen resultierenden Artefakten dieselbe Dichte aufwies, obwohl es keine Außenhaut besaß.

Serie 2: Steuerung von Dichte und Transparenz In der ersten Serie gab es keine Kollisionen von Fäden, da sie parallel zueinander verliefen und mit jeder Schicht die Richtung wechselten. In der zweiten Serie (Abb. 6-7) gab es auf jeder Schicht Linien, die sich in verschiedenen Bereichen willkürlich überkreuzten; dies führte zu einer radikal anderen Materialität, bei der die Dichte je Schicht variierte. Weil sich die Linien gelegentlich willkürlich überkreuzten, kam es zu Anhäufungen beziehungsweise zu lokalem Materialüberschuss.

Während dies zu einigen komplizierten Stücken führte, erlaubte die Zufälligkeit des Systems nicht genug Kontrolle über 


\section{6-7 Steuerung von Dichte und Transparenz}

die Materialität des Ergebnisses. Die Serie wurde weiterentwickelt, indem der Grad der Zufälligkeit abgewogen wurde, wobei ein Farbverlauf auf der Oberfläche des ,Mesh ‘ abgebildet wurde - je dunkler die Farbe, desto größer die Wahrscheinlichkeit, dass dort ein Strang beginnt. Der Algorithmus hat auch den Materialaufbau der unteren Schichten mit einbezogen. Zwar wurde die Transparenz nicht vollständig bestimmt, doch es konnten die Dichte des Materials sowie die Transparenz in Richtung einer bestimmten Dichte gelenkt werden.

Die Materialdichte und Transparenz auf dem Bildschirm abzubilden erwies sich als problematisch. Die hohe Dichte der Fäden würde sich auf dem Bildschirm zu einem einzigen farbigen Fleck verdichten. Die Dicke der Linie könnte manipuliert werden, um die Geschwindigkeit der Extrusion und somit die Menge an Material darzustellen, die aufgebaut wird. Das tatsächliche Fabrizieren war die einzige Möglichkeit, die Materialqualitäten des Ergebnisses zu testen.

Serie 3: Verschieben der Grenzen von Material und Maschine So wie alle digitalen Fabrikationsmaschinen hatte auch der Drucker ${ }^{18}$ Einschränkungen - die Größe des Drucks war limitiert, Formen mit Überständen konnten nicht gedruckt werden, er wies eine relativ geringe Geschwindigkeit auf und nur einige wenige Materialien in Form von Filament konnten verwendet werden. Die resultierenden Drucke wiesen klar Materialspuren des Druckprozesses auf - der flache Boden entsprechend des erhitzten Betts, die sichtbaren Schichten und die Spuren des Gewindeschneidens, wodurch im Modell instabile Überhänge auftraten. In der dritten Serie von 

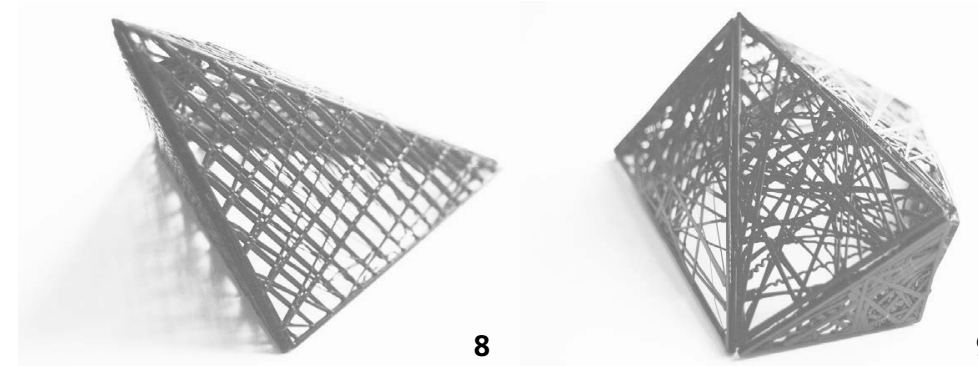

\section{8-9 Schraffieren mit Materie}

Objekten (Abb. 8-9) habe ich versucht, einige dieser Einschränkungen zu umgehen und bestimmte Merkmale der Maschine als Vorteil zu nutzen.

Anstatt ein fertiges Objekt zu drucken, habe ich mich dazu entschieden, Paneele zu drucken, die daraufhin zu größeren Objekten zusammengeführt werden können: Das digitale Modell wird dazu so groß skaliert, dass das größte Paneel gerade noch auf die Grundplatte passt. Die Paneele wurden eher flach gehalten, sodass die Druckzeit erheblich reduziert wurde. Aufgrund des erhitzten Betts bekamen die Paneele eine flache Unterseite und eine zweite, stärker ausgeprägte Seite. Beim Zusammenfügen können beide Seiten als Außenseite des Objekts verwendet werden, wodurch grundsätzlich verschiedene Artefakte in Bezug auf Material und Größe entstehen, jenseits dessen, was normalerweise mit diesen Maschinen hergestellt werden kann.

Die Algorithmen, die zum Füllen der Paneele verwendet werden, waren dieselben, die bereits für die Objekte ohne Haut sowie für die Objekte mit variierender Dichte getestet wurden. Der erste führte zu einer Schraffierung verschiedener Paneele und der zweite zu einer kontrollierten Dichte der Artefakte.

Serie 4: Eine zusätzliche halbe Dimension Ein dreidimensionales kartesisches Koordinatensystem ist die Basis für den im Entwurfsexperiment verwendeten Drucker. Eine zusätzliche Einschränkung besteht darin, dass eine Kollision mit dem abgegebenen Material verhindert werden muss. Die Bewegung des Druckerkopfes kann als 2,5D betrieben werden: Die Maschine legt je eine Schicht Material ab, indem sie sich in $\mathrm{x}$ - und y-Richtung 


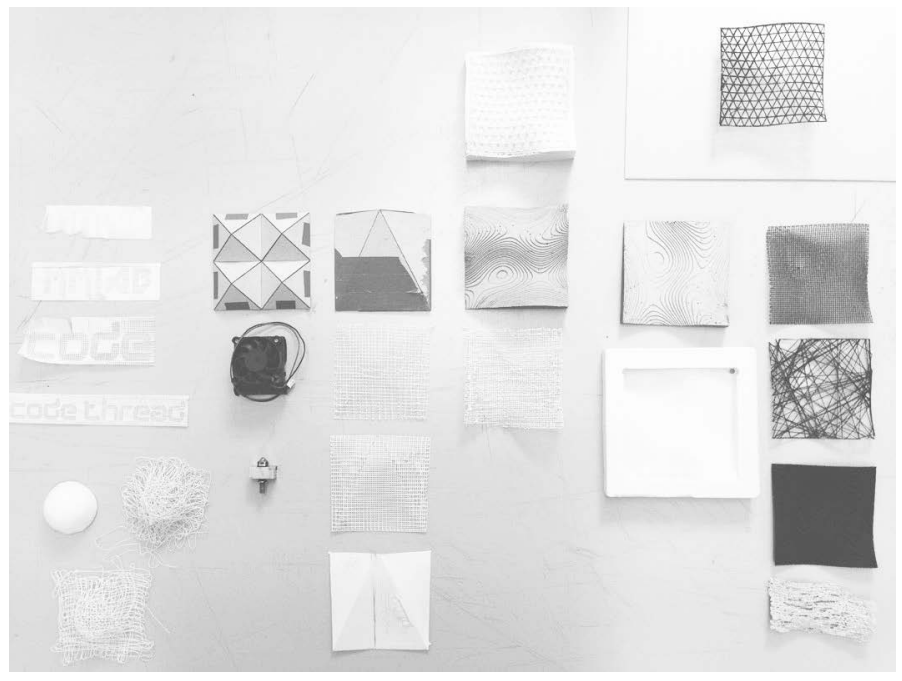

10 Experimentieren mit z-Richtung

bewegt, und in z-Richtung nur, nachdem eine Schicht fertiggestellt ist. Das Resultat dieses Prozesses ist in der geschichteten Struktur des gedruckten Artefakts sichtbar.

Eine letzte Serie von Artefakten (Abb. IO) versuchte diese Einschränkung zu umgehen, indem der Werkzeugkopf während des Druckprozesses in z-Richtung bewegt wurde und nicht zwischen den einzelnen Schichten. Das Druckerbett, die eigentliche Oberfläche, auf der die erste Schicht gedruckt wird, muss im normalen Druckprozess flach, waagerecht ausgerichtet und neutral sein, sodass der Druck leicht entfernt werden kann und keine Spuren hinterlässt. Aufgrund der zusätzlichen Bewegung in z-Richtung konnte ich auf dynamischeren Oberflächen drucken. Diese Freiheit brachte eine zusätzliche Komplexität ein: Wir mussten die digitale Datei an der zu bedruckenden Materialoberfläche ausrichten, um eine Kollision des Werkzeugkopfes mit dem bereits abgelegten Material zu vermeiden.

Während des Experiments wurde auf verschiedenen Oberflächen gedruckt, die durch das Schneiden von Karton und das Fräsen von Hartschaum hergestellt wurden. Die Herstellung der Formen resultierte in bestimmten Linien und Spuren: horizontale, topographische Linien im Falle des gestapelten Kartons und Spuren des Fräskopfs im Falle des gefrästen Hartschaums. Der erhitzte 
Kunststoff des Druckers passte sich diesen definierten Oberflächen an, was wirkungsvoll zu einer Materialität führte, die sowohl die Spuren der Gussform als auch die oben drauf gegossenen Kunststofffäden in sich trägt.

Das Drucken auf unebenen Oberflächen erforderte eine Anpassung der Oberfläche an die Bewegungen des Druckkopfes. Wenn ein paar Zentimeter über der Oberfläche gedruckt wurde, neigte das Material dazu, spiralförmige Fäden zu bilden. Durch versehentlich falsches Ausrichten der Oberfläche mit dem Druckkopf habe ich herausgefunden, dass sich die Spiralen allmählich anhäufen und sich bei langer Dauer des Extrudierens eine Grundlage formt, unabhängig von der Oberfläche, die sich darunter befindet.

Materialverständnis durch Code Neben den materiellen Artefakten (Abb. I I) brachte jedes der Experimente auch eine passend zugeschnittene Software hervor, die beim ,Processing " und in externen Bibliotheken geschrieben und später in Grasshopper und Rhino übersetzt wurden. Die Software ermöglichte algorithmische Untersuchungen von Form- und Fabrikationsparametern, bot ein visuelles Feedback, um die Ergebnisse zu bewerten, und verfügte über Funktionen zum Importieren und Exportieren von Dateien. Diese Entwurfswerkzeuge wurden entwickelt, während die Entwürfe vorangetrieben und die Artefakte hergestellt wurden. Durch das Entwerfen dieser Serien und die zugehörigen Algorithmen hat sich ein Verständnis für Anforderungen und Widerstände auf unterschiedlichen Ebenen entwickelt: ein computerbasiertes Verständnis für die Codes und Dateien sowie ein Verständnis für Möglichkeiten des Materials oder für Beschränkungen in der Herstellung.

Die verschiedenen Arten mit Code, Maschine und Material umzugehen sowie das verschiedene Feedback zwischen diesen und dem Designer zeigten einen gestuften und nicht-linearen Entwurfsprozess auf. Auch wenn sich das Verständnis sukzessive entwickelt hat, war es kein kontinuierlicher Prozess: Manipulationen am zugrundeliegenden Algorithmus erforderten ein Anhalten, Ändern und Neustarten des Codes, Untersuchungen der Veränderungen durch Tests an verschiedenen Parametern sowie die Steuerung des digitalen Modells - bewertet mithilfe von Bildern am Bildschirm. Sobald dies zu einem vielversprechenden Stand führte, wurde der Prozess gestoppt, ein Bild gesichert, eine Datei exportiert und der 


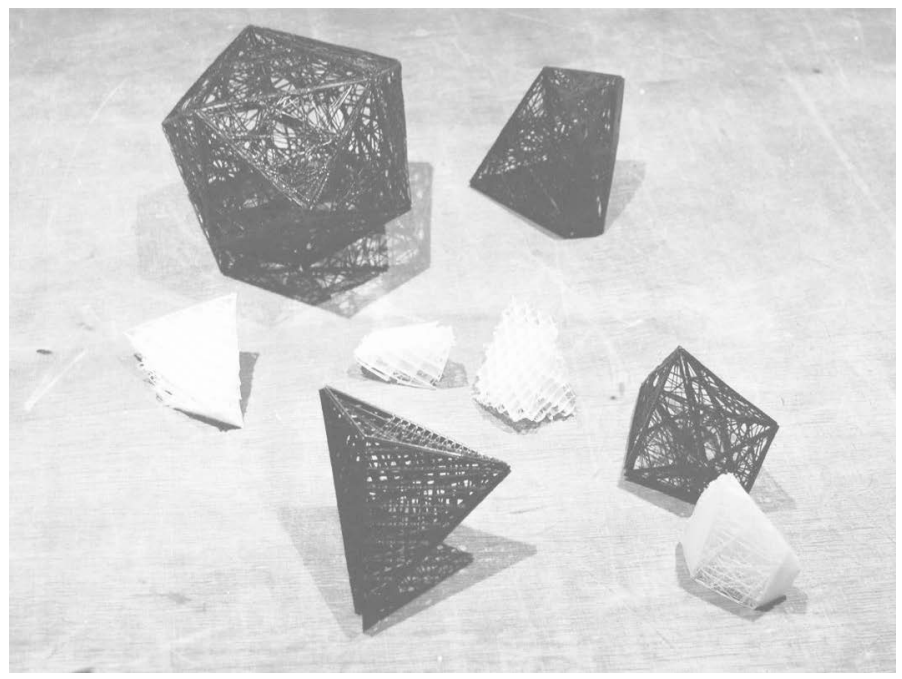

11 Sammlung fabrizierter Artefakte

Code in der Datei in einem Texteditor geprüft, bevor er endgültig an den Drucker gesendet wurde. Der Druckprozess wurde beobachtet, wenn möglich wurde eingegriffen und ein Impuls gegeben und nach Auswertung der Ergebnisse konnte der Prozess wieder von Neuem gestartet werden.

Fazit: Hacking Agency Die Forschung, die in diesem Beitrag behandelt wurde, geht von einem Interesse an digitaler Fabrikation als spezifische Herstellungsweise aus, die zwischen dem Digitalen und dem Materiellen, zwischen dem Entwurf und der Herstellung sowie zwischen dem Abstrakten und dem Körperlichen agiert. Das im Vorigen erläuterte Projekt Encoded Matter untersucht genau die unterschiedlichen Schritte in der Entwicklung von einem digitalen Modell zu einem materiellen Artefakt unter Verwendung einer bestimmten digitalen Fabrikationstechnologie. Während der Umfang von Encoded Matter und die Größenordnung der produzierten Artefakte limitiert sein mögen, veranschaulicht die Forschung, dass ,computation ' und digitale Fabrikation keine neutralen oder transparenten Technologien sind; sie beeinflussen im Gegenteil, was entworfen und hergestellt werden kann. Die ,agencies', die in Material und Code gefunden wurden, spielen in dem Prozess der Herstellung im Rahmen digitaler Fabrikation eine 
Rolle und werden darüber hinaus von solchen agencies ergänzt, die in Fabrikationsmaschinen gefunden wird. Das Hacking ermöglicht diesen, agencies ‘ vermittelt zu werden.

Das Material, das in den Prozessen digitaler Fabrikation in dieser Forschung verwendet wurde, ist äußerst standardisiert und industriell hergestellt; diese Kontrolle des Materials wird zusätzlich durch den Prozess digitaler Fabrikation verstärkt. Die Forschung zeigt, dass eine dem Material zugeschriebene Agency im Spiel ist. Ein materielles Artefakt herzustellen bleibt bis zu einem gewissen Grad unvorhersehbar, auch wenn dies mithilfe stark kontrollierter, mechanischer Prozesse digitaler Fabrikation geschieht. Während der Entwurfsuntersuchung bin ich auf viele Fälle solchen Materialverhaltens gestoßen, die in den Dateien oder im Fabrikationsprozess nicht vorhersehbar waren, um Beispiel die Verzerrungen, das Triefen des Filaments oder die Gebilde geschmolzenen Kunststoffs.

Ein Verständnis des Codes hilft, die Technologien zu verknüpfen, mit ihnen herumzubasteln und sie zu hacken. Digitale Technologien sind hochentwickelte, kulturelle Artefakte, die sich tendenziell schrittweise entwickeln und sukzessive auf vorigen Codes aufbauen. ${ }^{19}$ Die Geschwindigkeit, mit der sich digitale Technologien entwickeln, scheint immer schneller zu werden. Wir müssen uns lediglich anschauen, wie sich das RepRap-Projekt seit dessen Anfängen im Jahr 2005 sowohl qualitativ als auch quantitativ weiterentwickelt hat, genauso wie meine erste Version, die ich 2009 gebaut habe, und wo wir heute stehen: Die Anzahl an Druckern, Vertrieben und Firmen, die auf RepRap aufbauen, ist schwierig nachzuverfolgen. Bei der Suche nach Agency in diesen Technologien finden wir einen Teil von ihnen in diesen schrittweisen, kollektiv entwickelten Codes, welche die Technologien antreiben.

Die in diesen Entwurfsuntersuchungen verwendeten Fabrikationsmaschinen und die Fabrikationsprozesse, die sie ermöglichen, hinterlassen ihre eigenen Spuren in den hergestellten Artefakten: die sichtbare geschichtete Struktur sowie die Einschränkungen der Größe in beiden Drucktechnologien, der flache Boden entsprechend des erhitzten Betts, die Unmöglichkeit, Überhänge zu drucken sowie die sichtbaren Materialfäden in den Serien des Encoded Matter-Projekts. Die Maschinen, als vom Code gesteuerte materielle Apparate, übernehmen Aspekte und Qualitäten sowohl 
vom Material, mit dem sie arbeiten, wie auch vom Code, der sie steuert. Darüber hinaus werden durch die Verwendung eines digitalen Codes als Input und entwickelter materieller Objekte als Output Spuren des Codes in der materiellen Welt sichtbar. Anstatt die Faser, die Auflösung und die sichtbaren Spuren der Technologie als Problem zu betrachten oder als etwas, das durch eine bessere, neuere Version der Technologie gelöst werden muss, können wir für und mit diesen Eigenschaften entwerfen.

\section{Anmerkungen}

1 Vgl. Alberto Perez-Gomez und Louise Pelletier, Architectural Representation and the Perspective Hinge, Cambridge, Mass. 2000 sowie Robin Evans, The projective cast. Architecture and its Three Geometries, Cambridge, Mass. 1995.

2 Kester Rattenbury, This Is Not Architecture. Media Constructions, London, New York 2002.

3 Robin Evans, "Translations from Drawing to Building”, in: Ders., Translations from Drawing to Building and Other Essays, London 1997.

4 Vgl. Greg Lynn, Animate form, New York 1999 und Centre Georges Pompidou (Hg.) Architectures non standard, Ausst.-Kat. Centre Georges Pompidou, Paris 2004.

5 Stephen Kieran und James Timberlake, Refabricating Architecture. How Manufacturing Methodologies Are Poised to Transform Building Construction, New York 2004, S. 57.

6 Mario Carpo, The Alphabet and the Algorithm, Cambridge, Mass. 2011, S. 78.

7 Die erste Iteration dieses Projekts wurde entwickelt in Generator.x 3.0. From Code to Atoms, Meisterklasse mit Marius Watz, am iMal Brüssel, 2012. Siehe: www.imal.org/nl/activity/generatorx3 [12. Mai 2015].

8 Corneel Cannaerts, Negotiating Agency. Computation \& Digital Fabrication as Design Media, Doctoral Thesis, RMIT Melbourne 2015, https://researchbank.rmit.edu.au/eserv/rmit:161310 [13.05.2019].

9 Während viele Beispiele für Architekten und Designer mit besonderem Fokus für diese Spuren genannt werden können, verweise ich hier auf das Buch von Jonathan Hill, Actions of Architecture. Architects and Creative Users, New York 2003 sowie Ders., Immaterial Architecture, New York 2006; siehe auch: Tim Ingold, Making. 
Anthropology, Archaeology, Art and Architecture, London, New York 2013.

10 Tim Ingold, Making (Anm. 9). S. 47f.

11 Es gibt Forschungen über das Scannen von natürlichen Materialien für den späteren Gebrauch mit digitaler Fabrikation, wie z. B. The Woodchip Barn entwickelt von der AA im Hooke Park.

Siehe: www.designandmake.aaschool.ac.uk/woodchip-barn/ [20. Juni 2016].

12 Neil A. Gershenfeld, Fab. The Coming Revolution on Your Desktop, from Personal Computers to Personal Fabrication, New York 2007.

13 Siehe: www.reprap.org [20. September 2015].

14 Siehe speziell die Nutzung von Farb-3D-Drucken für die Dazzle Lamp Prototypen. Siehe: Corneel Cannaerts, Fabricating Material Intensities. Designing and Making Mediated by Computation, Aarhus 2015, S. 152-161.

15 Die Technologie wird auch, Fused Deposition Modelling' genannt, doch diese Bezeichnung ist von dem Unternehmen Stratatsys geschützt, die als erstes diese Technologie entwickelt haben. Erst seit dem Auslaufen der ursprünglichen Patente für die FDM-Technologie hat der Gebrauch von 3D-Druckern zugenommen. Siehe: www.reprap.org/wiki/Fused_filament_fabrication [24. Oktober 2015].

16 Für eine genaue Definition der g-Codes, siehe: www.reprap.org/wiki/G-code [26. September 2015].

17 Siehe: www.blog.diatom.cc/category/codethread [1. September 2014].

18 Mehrere Drucker wurden benutzt: Ein, Makerbot' und ein ,Ultimaker'.

19 G-Codes wurden ursprünglich vom MIT Servomechanisms Laboratory entwickelt, siehe: www.libraries.mit.edu/mithistory/research/ labs/mit-servomechanisms-laboratory/ [15. März 2016]. 
Christophe Barlieb

\section{Cybercraft: Das neue Paradigma}

Die Gegebenheiten, die durch die zunehmende Annäherung von Mensch-Maschine-Materie und wissenschaftlicher Forschung entstehen, definieren neu, wie wir Dinge wahrnehmen, mit ihnen interagieren und sie herstellen. Generische Sensoren, Rechenleistung und drahtlose Netzwerktechnologien in unseren Körpern, Materialien, Häusern, Schulen und Arbeitsplätzen prägen die ,Mainstream'-Kultur neu. KI-Agenten (Künstliche Intelligenz) verarbeiten vernetzte Sensordaten in Echtzeit gegen bisherige, aktuelle und prognostizierte Modelle und liefern Informationen über Entscheidungen und Maßnahmen, deren Auswertung einst viel Zeit in Anspruch nahm. Diese technologischen Phänomene, kombiniert mit realen ökologischen und demographischen Veränderungen, greifen in bisher nicht vorhandener Größenordnung und Geschwindigkeit in die evolutionären Prozesse ein. Roboter, die einst ausschließlich mit der industriellen Serienmontage verbunden waren, fertigen individuelle Dinge, transportieren und montieren sie autonom. Handcraft und Digitalcraft fusionieren zu Cybercraft und vernetzen Menschen, Maschinen und Materie. Diese anhaltenden Phänomene verändern zwangsläufig die 600 Jahre alten Entwurfsmethoden der Architektur und die elf Jahrtausende alten Baupraktiken.

\section{Einführung}

„,Je mehr der Künstler über seine Kunst nachdenkt, desto mehr versucht er, den Ausdruck zu perfektionieren, mit dem er seine Bedeutung interpretieren würde, desto mehr wird er dazu gebracht, den ursprünglichen Ausdruck zu verstärken, um ihn klarer zu machen“.

Viollet-le-Duc ${ }^{\mathrm{I}}$ 
Menschen, die mit digital vernetzten Sensoren, Maschinen und Materialien interagieren, definieren die Cyber-Ära. Das ,nahtlose“ Geflecht zwischen analogen und digitalen Medien macht diese Ära einzigartig. Verkörperte Intelligenz, die in interdisziplinärer Forschung und Praxis in der Robotik aufgeht, verändert die Art und Weise, wie wir Dinge wahrnehmen, mit ihnen interagieren und sie gestalten. Diese Fortschritte, die von kostengünstigen generischen Sensoren, Cloud Computing und Robotern angetrieben werden, definieren Theorie, rechtliche Rahmenbedingungen und Werkzeuge der Architektur neu. Aus innovativen Mensch-Maschine-Interaktionen, Sensortechnologien, künstlicher Intelligenz und Fortschritten in der Materialtechnologie entwickeln sich neue Architekturen.

Die folgenden Gedanken befassen sich mit den sogenannten Werkzeugen der Architektur, den bisherigen digitalen Praktiken auf diesem Gebiet und dem Status quo. Ein neuer Wissenshorizont zeichnet sich ab. Drei beispielhafte Praktiken des Cybercraft werden analysiert, um Annahmen über zukünftige Architekturentwurfsstrategien und die damit verbundenen Medien in ihrer Agency treffen zu können. Das erste, Convective Apartments, beschäftigt sich mit Meteorologie und Physiologie als Gestaltungsmedien. Während die zweite, Mesh Mould Metal, die In-situ-Fertigung mit Robotern untersucht. Schließlich nutzt Adaptive Robotic Carving maschinelles Lernen (ML) bezogen auf das Materialverhalten, um Mensch-Maschine Handlungsprozesse für die Holzverarbeitung nutzbar zu machen.

Diese Forschungsarbeiten befassen sich mit Architekturdesign, Ressourcenmanagement, autonomen Bauweisen, intelligenten Mensch-Maschine-Interaktionen, Materialverhalten sowie Fragen der analogen und digitalen Medien. Die Verhandlung der globalen und lokalen Herausforderungen des Klimawandels und des demographischen Wandels erfordert eine vertiefte Untersuchung des systemischen Netzes von Menschen, Maschinen und Materie. ${ }^{2}$

Drei Werkzeuge Architekten und Architektinnen vermitteln die räumliche und elementare Beschaffenheit der Architektur mittels dreier Werkzeuge aus der Renaissance. ${ }^{3}$ Das erste Werkzeug ist eine schriftliche Erläuterung der architektonischen Absichten und Besonderheiten. Die zweite ist ein zweidimensionaler Satz maßstäblicher Zeichnungen, der von Projektübersichten bis hin zu detaillierten räumlichen Dimensionen und Beziehungen von Archi- 
tekturelementen untereinander und zu deren Umgebung reicht. Die letzte ist eine Variation von maßstäblichen physischen Modellen, die räumliche, materielle und bauliche Eigenschaften wiedergeben. Diese drei Werkzeuge helfen Entwerfenden, zu forschen, Intentionen zu formulieren und mit Bauherren, Behörden und Auftragnehmern zu kommunizieren. Sie bilden in der Regel rechtsverbindliche Architekturdokumente. ${ }^{4}$ In den letzten 6o Jahren haben sich diese etablierten Praktiken und Methoden zunehmend von analogen zu digitalen Medientechnologien gewandelt.

Neue Architekturkonstruktionen und Darstellungsmodalitäten ergaben sich aus diesem Wandel. Die heutigen digitalen Architekturmodelle beschränken sich nicht länger auf die Darstellung. Digitale Modelle sind Daten, die mit logischen Entwurfs-, Herstellungs- und Konstruktionsmethoden verknüpft sind. Die Methoden des Entwurfes auf dem Gebiet der Architektur werden durch die ,Robotisierte Konstruktion ' verändert. ${ }^{5}$

Autonome Architektursysteme sind in Zukunft wahrscheinlich und werden durch das Verbinden von analogem Wissen, das durch Generationen von ,Handwerkskunst' gesammelt wurde, mit digitaler Sensorik-, Computer- und Bautechnologie ermöglicht. ${ }^{6}$ Meines Erachtens bemerkt Chaikin zurecht, dass diese Entwicklungen zu langsam vorangehen. ${ }^{7}$ Denn derzeit befassen sich nur eine Handvoll Graduierten- und Postgraduiertenprogramme kritisch mit den technologischen Veränderungen der digitalen Architektur. Dabei ist vor allem wichtig, diese Werkzeuge nicht nur als Instrumente zu lesen, sondern sie vielmehr als performative Medien zu begreifen.

Digitale Rückblende In den späten I960er Jahren führten Jörg Schlaich und Rudolf Bergermann weiterführende digitale Berechnungen in die Konstruktionstechnik ein, um die anspruchsvolle Kabelnetzstruktur des Olympiastadions München zu berechnen. ${ }^{8}$ Bereits Ende der I970er Jahre wurden die digitalen Informationstechnologien zum Mainstream. Softwareentwickler lieferten digitale Versionen traditioneller Entwurfswerkzeuge für Architekten und Architektinnen, um Informationen intuitiv erstellen, bearbeiten, kopieren, einfügen und löschen zu können. Architekten konnten endlose Bibliotheken von Details, Komponenten und räumlichen Konfigurationen digital speichern, umfangreiche Stücklisten und Zeitpläne verwalten. Mit wenigen Tablet- oder Mausklicks verwan- 
delten Architekten Ideen schnell in gedruckte Pläne und Gebäude. Die allgemeine Mentalität des Konstruierens von Architektur mit digitalen Medien verblieb allerdings auf dem Stand des analogen Zeichnens mit Zeichenschiene und Reißbrett. ${ }^{9}$ Monges Darstellende Geometrie und Albertis Beschreibungen zur Perspektive reichten aus, um postmoderne Architekturvisionen zu entwickeln und zu vermitteln. Eine junge Architektengeneration erkannte bald, dass computergestütztes Design (CAD) eine neue Technologie war, die so nur eine alte Methode lediglich stütze. ${ }^{\text {Io }}$ Wenn Architektur sich weiterentwickelte, entweder aufgrund neuer Bautechnologien oder neuer Darstellungsmodalitäten, dann leistete CAD zu keinem der beiden einen Beitrag. ${ }^{\text {I }}$

Zur gleichen Zeit entwickelten Luft- und Raumfahrt-, Automobil- und Infrastrukturingenieure neue digitale Design- und Fertigungswerkzeuge und rationalisierten so die Entwicklungs- und Fertigungsprozesse. Der Architekt Frank Gehry hat die Medienpraxis der Architektur radikal beeinflusst. Anfang der I 99oer Jahre führte er neue digitale Technologien und Arbeitsprozesse ein, um komplexe gekrümmte Oberflächen, deren Messdaten von Papiermodellen abgenommen wurden, zu bewerten, $\mathrm{zu}$ rationalisieren und herzustellen. Gehry hat Modelle digitalisiert, um ,NURBS ‘Oberflächen parametrisch bearbeiten zu können. Schließlich gründete er „Gehry Technologies“, um Werkzeuge zu entwickeln, die diese neuen konstruktiven Geometrien in der Baupraxis realisierbar machten. Aus diesem Prozess sind grundlegende Standards für die heutige ,Building Information Modeling (BIM) und ,File-toFactory'-Kultur in der Architektur geschaffen worden.

Mitte der I99oer Jahre löste die Einführung des Personal Computers (PC), eines preiswerten und allgemein verfügbaren Computers, ein Wettrüsten der Hard- und Software-Hersteller und -Anbieter aus. Mathematiker entwickelten und implementierten neue Algorithmen, unter anderem für ,texture shaders', geometrische Bearbeitungen und ,Computer Vision" (CV). Eine neue Generation experimentierte auf dem Gebiet der digitalen Medientechnologien, während einige Architekten vor möglichen Defiziten dieser Art von Architektur warnten. Risse im Architekturdiskurs, ebenso wie ,neue Schulen“ entstanden, die das konventionelle Designdenken herausforderten. ${ }^{\mathrm{I} 2}$ Computergrafik-(CG)-Tools wurden zur Indexierung von Formen in virtuellen zeitbasierten Systemen eingeführt, die ihre Umsetzung mit computergestützten numerisch 
gesteuerten (CNC) Maschinen ermöglichten. ${ }^{13} \mathrm{Zu}$ dieser Zeit war die physische Erstellung dieser neuen Formen eine Herausforderung; Werkzeuge und Arbeitsabläufe mussten erst entwickelt werden.

Schließlich, etablierte sich das Internet mit der Jahrtausendwende als neuer Wissensvermittler, der es den Menschen ermöglichte, Informationen auf globaler Ebene unmittelbar zu erhalten und zu teilen. Diese Art der Netzwerkkommunikation führte zusammen mit dem Phänomen des ,Bilbao-Effektes“ und des ,Starchitekten “ $\mathrm{zu}$ einer Entwicklung von Abhängigkeiten in der Architektur. Peking wurde während der Zeit des wirtschaftlichen Aufschwunges und der Zeit der Vorbereitung der olympischen Spiele zu einer Heimat von internationalen Experten. Große Kapitalbeträge in Verbindung mit extremem Zeitdruck führten zur vollständigen Herausbildung und Durchsetzung der Methoden und Technologien des digitalen Entwerfens. Parametrische Entwurfswerkzeuge ermöglichten es den Arbeitsteams, während des gesamten Konstruktionsprozesses mit ,Smart Geometry ${ }^{6}$ zu arbeiten. ${ }^{14}$ Die Architekten arbeiteten im Takt zwischen Asien, Europa und Nordamerika. Digitale Dateien von Plänen und Modellen waren rund um die Uhr zugänglich. Die Architekten, die vormals für ihre vermeintlich nicht realisierbaren Architekturen belächelt wurden, konnten nun rund um die Welt ihre ,Blobs' verwirklichen. Poetische Computergrafik-Bilder wie von Philipp Schaerer gaben neue Impulse für einen weiterführenden Diskurs auf dem Gebiet der Architekturvisualisierung, während Arnold Walz' elegante Skriptmethoden es Stararchitekten ermöglichte, schwierige Geometrien durch systematische Vereinfachung der Komplexität zu kontrollieren. Das Credo ,I do it because I can " ist mit der Finanzkrise 2008 schließlich zusammengebrochen. Im selben Jahr präsentierte das MoMA eine neue Generation von Architekten, Designern und Künstlern der digitalen Medien. ${ }^{\mathrm{I}}$

In den letzten zehn Jahren gab es eine Flut in der Entwicklung von digitalen Werkzeugen und visuellen Programmierschnittstellen, die unter anderem auf Konferenzen wie „Smart Geometry“, „Advances in Architectural Geometry“ und „Robots in Architecture“ vorgestellt wurden. ${ }^{16}$ Preiswerte digitale Modellierungstools und visuelle Programmierschnittstellen bereiteten neue Wege und knüpften Verbindungen zwischen den ,Design-Communities . 
Status quo Heute arbeiten Architekten und Architektinnen, die fließend in Programmiersprachen wie $\mathrm{C}++, \mathrm{C \#}$, Python, Java und JS sind, auf Open-Source-Plattformen zusammen, entwickeln gemeinsam ,Tools' und teilen versionskontrollierte Quellcodes, um sie offen für alle zu teilen, zu verwenden, zu klonen, zu prüfen, $\mathrm{zu}$ publizieren und zu verbreiten. Digitale Medientechnologien durchdringen die Architektur in einem gewaltigen Tempo, und die akademischen Lehrpläne passen sich nur langsam an. ${ }^{17}$ Neue Entwurfsmethoden, die an der ETH Zürich, CITA und MIT entwickelt wurden, fordern traditionelle Praktiken heraus. Sie werden im Rahmen interdisziplinärer Forschungsprojekte in den Bereichen Fertigung, Materialverhalten und Medientechnologie erforscht. Die Anwendung dieser neuen Forschungsthemen und -modelle auf die Architektur- und Bauindustrie erfordert eine starke interdisziplinäre Zusammenarbeit. ${ }^{18}$ Architektenkammern, Akkreditierungsstellen und Universitäten müssen diesen neuen Praktiken und Anforderungen entsprechend die Lehrangebote überarbeiten, um zukünftige Architekten und Architektinnen unterrichten und fördern zu können. Im Folgenden werden diese aktuellen Themen im Hinblick auf das Erfassen, Anpassen und Gestalten von Architektur im Zeitalter des Cybercraft reflektiert: ein Raum-Zeit-Phänomen, bei dem Analog und Digital hybrid ineinandergreift, das Mensch, Maschine und Materie miteinander vernetzt.

Neuer Wissenshorizont Seit den Anfängen der europäischen Moderne, die sich in Holland, Deutschland und Frankreich vor Ioo Jahren entwickelt hat, haben wir nicht mehr eine so beeindruckende Bandbreite innovativer Entwurfspraktiken auf dem Gebiet der Architektur erlebt. ${ }^{19}$ Architekten und Architektinnen kodieren den Raum von Ferne, kodieren Materialien und bauen Architekturen autonom auf. ${ }^{20}$ Hybride analoge und digitale Medien vernetzen Menschen, Maschinen und Materialien und ermöglichen EchtzeitEntwurfs-Prozesse. Kostengünstige Sensorik, computergestützte Bearbeitung und Fertigungstechnologien führen zur Anbindung digitaler Medien, während Sensoren, Prozessoren und Manipulatoren, die in Feedback-Schleifen integriert sind, Handlungsspielraum erzeugen.

Entstehen intelligente Multiagentensysteme? Oliver Brock argumentiert, dass Intelligenz erkennbar ist, wenn wir sie sehen. Sie ist quantifizierbar, aber wir können sie nicht definieren. Um 
das Problem zu lösen, schlägt Brock vor, unter anderem Maschinenintelligenzen $\mathrm{zu}$ erforschen, um die menschliche Intelligenz $\mathrm{zu}$ erfassen. ${ }^{2 \mathrm{I}}$ Unsere taktilen, visuellen, akustischen, olfaktorischen und geschmacklichen Organe liefern Signale an unser Gehirn, um Phänomene wahrzunehmen. Nach der Prozessierung aktivieren bioelektrische Impulse die Muskeln und informieren unsere Umwelt. Die mathematische Modellierung dieses Prozesses ist vergeblich - wie können wir 300 Millionen Nerven, Ioo Milliarden Gehirnzellen, 6oo Muskeln modellieren? Ist das überhaupt notwendig? Der Mensch zeichnet sich dadurch aus, die wahrnehmbare Welt zu abstrahieren, zu simulieren, darzustellen und zu mechanisieren.

Zerstörungsfreie Sensorik ermöglicht die Erforschung des Weltraums und der oberflächennahen Geologie, die vom Niederfrequenzradar bis zum Hochfrequenzultraschall reicht. ,MultiSensing ' erzeugt ein Spektrum von messbaren Phänomenen. So können beispielsweise vernetzte Sensoren (Temperatur, Luftdruck, Luftfeuchtigkeit, Luftpartikel, Schalldruck, Umgebungslicht) an statischen Orten auf der Welt klimatische Ereignisse erfassen und sichtbar machen. Mit dem Wachstum der Datensätze und der Entwicklung analytischer Methoden werden zeitabhängige Algorithmen zur Erkennung von Mustern eingesetzt. Das Earth-Projekt ist ,eine Visualisierung der globalen Wetterbedingungen, die von Supercomputern prognostiziert wird, die alle drei Stunden aktualisiert werden. Die aktuellen Schätzungen der Meeresoberfläche werden alle fünf Tage aktualisiert, während die Temperaturen der Meeresoberfläche und die Anomalie des täglichen Durchschnitts ( I98 I-20 I I) täglich aktualisiert werden. Die Meereswellen werden alle drei Stunden aktualisiert, und Aurora aktualisiert alle dreißig Minuten“.22 Das Smart Citizen Kit ist eine weitere von mehreren Open-Source-Plattformen, die allgemeine Klimadaten für Forscher und Designer bereitstellen. ${ }^{23}$ Über diese Plattformen können Architekten und Architektinnen Stadt- und Landschaftsprojekte planen, die an sich entwickelnde Klimamodelle angepasst sind.

,Simultaneous Localization and Mapping“ (SLAM), ,Laser Detection, and Ranging (LIDAR) und Wärmesensoren vermessen den Raum lokaler Umgebungen zur Kartierung in Echtzeit. Das Ergänzen der Sensoren um das ,Global Positioning Systems، (GPS) erhöht die Genauigkeit der Ortung. Einst für die Luft- und Raumfahrt und den militärischen Einsatz entwickelt, sind diese Tech- 
nologien heute allgemein verfügbar. Sie kommen in bestehenden Fahrzeugen und Baumaschinen vor, unter anderem in Smartphones, Autos, Shuttles, Inspektionsdrohnen, Roboterkränen, Mauerrobotern.

Sensoren sammeln genaue Daten. So kann beispielsweise eine Stereokamera Tiefe, geometrische Merkmale, Farbbandbreite und aktuelle Positionen erfassen. Der endlose Datenstrom der Kamera allein ist nutzlos. Die Daten werden erst nach der Verarbeitung lesbar. Die Kombination verschiedener Datenströme von Sensoren ermöglicht es Phänomene zu visualisieren, die unsere Sinne zum Teil wahrnehmen und zum Teil auch nicht wahrnehmen können.

Sensordaten in Kombination mit Cloud Computing und intelligenten Algorithmen unterstützen KI-Systeme. Feedbackschleifen zwischen Datenerfassungstechnologien und Prozessagenten sorgen für spannende Ergebnisse mit großem Potential. Diese Paradigmen stellen herkömmliche Entwurfsmethoden in Frage, indem sie die Art und Weise, wie wir Dinge wahrnehmen, mit ihnen interagieren und sie gestalten neu definieren.

Vernetzte Computer können trainiert werden, Muster zu erkennen, die Menschen niemals in der Lage wären zu unterscheiden. ,Computational Agency und Anpassung sind Schlüssel zu neuen Methoden des Entwurfes. Die Anpassung ist ein Hybrid aus Computer und Kommunikation, der eine perzeptuelle Veränderung ermöglicht, die auf dem ursprünglichen sensorischen Input aufbaut. Dies wiederum führt zu entsprechenden Handlungen, die unsere Lebensräume gestalten können.

Das Open-Source-Robot-Operating-System (ROS) bildet das Herzstück der derzeit meisten Roboterinnovationen in der Architekturentwicklung und -fertigung. ${ }^{24}$ Der ROS-Kern ist für die Kommunikation zwischen Knoten zuständig, die Dienste über das digitale TCP/IP Handshaking veröffentlichen und abonnieren. Knoten sind Hard- und Softwaresysteme, die an ROS ,hängen'. Robotische Vorrichtungen und Sensoren (Hardwareknoten) übertragen Daten über das ROS-Netzwerk, während Anwendungen (Softwareknoten) die Daten verwenden, um Bewegungen zu planen, Objekte zu erkennen und Entscheidungen zu treffen. Praktisch jedes Gerät und jede Software kann sich über eine kompatible C++-, C\#-, Python-, Java- und/oder JS-Schnittstelle an ROS anschließen. Da ROS über TCP/IP kommuniziert, ist es möglich, Anwendungen auf zahlreichen Betriebssystemen auszuführen. Die Steuerung von Roboter- 
systemen direkt über webbasierte Applikationen und interaktive grafische Benutzeroberflächen (GUIs) machen ROS zu einem vielseitigen Vermittler für mobile und flexible Anwendungen.

Kraft-Moment-Sensorik in Maschinen ermöglicht haptische Wirkungen. Menschen nutzen Druck und taktile Wahrnehmung, um zu spüren, wie die Dinge zusammenpassen. Roboter wie der $S A R A$ des DLR können ein sub-Newton Kraft-Moment messen. ${ }^{25}$ Diese nachgiebigen Roboter ermöglichen eine neue Zusammenarbeit zwischen Menschen und Maschine ohne herkömmliche Roboterzellengehäuse. Menschen können physisch mit diesen Maschinen interagieren, um entweder dabei zu helfen, unvorhergesehene Bedingungen zu überwinden oder ihnen spezifische Konfigurationen zu vermitteln.

Kraft-Drehmomentsensoren, die in anpassungsfähige Roboter eingebaut sind, die hochauflösende Vektorkraftdaten über ROS publizieren, ermöglichen komplexe Montagen. In Kombination mit CV erkennen diese Maschinen Hindernisse, Teile und Ziele in kürzester Zeit. KI, die auf den entsprechenden Maschinen läuft, gibt an die Roboter Informationen zurück, die ihnen die weitere Aktion im Raum ermöglichen. Die Anpassung an sich ändernde Umgebungsbedingungen in Echtzeit bedeutet einen Paradigmenwechsel in der Fertigung. Das bedeutet, dass generische Maschinen nun mittels Datenströmen, übergreifend gefassten Aufgaben und Feedbackschleifen agieren.

Die folgenden exemplarischen Beispiele verdeutlichen die Interaktion zwischen Mensch, Maschine und Materie sowie das Lernen mittels Sensortechnologien und angepassten ML-Feedbackschleifen zur Herstellung neuer architektonischer Materialien, Elemente, Räume und Ästhetiken.

\section{Drei Praxisbeispiele}

Convective Apartments Philippe Rahm, ein Schweizer Architekt, definiert seine Praxis als eine Mischung aus Architektur, Ingenieurwesen und Forschung. Er unterrichtet an der Nationalen Schule für Architektur in Versailles.

In seinen Experimenten bilden meteorologische und physiologische Phänomene konstruktive Mittel. ${ }^{26}$ Das Projekt Convective Apartments nutzt das physikalische Gesetz des Luftauftriebes, um das Klima von Wohnumgebungen zu regulieren: weniger 
dichte, warme Luft steigt auf, während dichtere, kalte Luft fällt. Rahm entwickelt Klimazonen, die auf menschlichen und maschinellen Aktivitäten basieren, um den Wärmeaustausch zu steuern. Er schlägt zum Beispiel die Wärmeregulierung des menschlichen Körpers durch Kleidung vor, um den Energieverbrauch in nur geringfügig und auch umgekehrt in stark frequentierten Räumen zu verringern:

„Ein intelligentes System auf Basis von Temperatursensoren, die außerhalb des Gebäudes und im öffentlichen Raum installiert sind, misst die Temperatur in Echtzeit. [...] Die Software berechnet die genaue zu ersetzende Luftmenge für jede Wohnung in Bezug auf die Anzahl der Bewohner und die durch ihre Aktivitäten erzeugte Abluftmenge, um den Energieverbrauch zu senken“. ${ }^{27}$

Diese Methode der Thermoanpassung wird durch Sensorik und räumliche Konfiguration ermöglicht. Die Decken des Gebäudes werden zu Klimazonen, verformt‘. Die verschiedenen Geschosshöhen, kombiniert mit Sensordaten, regeln den Luftstrom durch die Räume. Diese klimatischen Gestaltungsstrategien beziehen Nutzer und räumliche Nutzungen ein, um ihre Grundrisse zu gestalten und zu strukturieren sowie das formale Erscheinungsbild des Gebäudes zu beeinflussen. Rahm verwandelt im Wesentlichen ein physikalisches Phänomen, die Wärme, in eine visuelle, thermische Landschaft".

Die Erforschung sinnlicher Phänomene zur Generierung von Architektur durch die Vernetzung von aktiven und passiven Sensortechnologien, die von der KI gesteuert werden, könnte zu einer wesentlichen Reduzierung des Energiebedarfs führen. Durch die Kombination verschiedener Gebäudesystemtechnologien mit erneuerbaren Baustoffen und intelligenten Bauweisen entstehen neue Formen von ,intelligenter' Architektur.

In diesem Zusammenhang fordert Rahm: ,Wir müssen unseren Fokus von einem rein funktionalen Ansatz auf einen sensibleren und auf unsichtbare, klimabezogene Aspekte des Raumes achten". ${ }^{28}$

Mesh Mould Metal Fabio Gramazio und Matthias Kohler (GKR) von der ETH Zürich sind Pioniere bei Entwurfsmethoden der robotergestützten Architektur. Ihr erstes Roboter-Experiment zum 
Mauerwerk zeigte, dass ein generischer industrieller Manipulator (Roboter) maßgefertigte architektonische Elemente herstellen konnte. ${ }^{29}$ Der Roboter ist damit kein reines Serienwerkzeug mehr.

Ihre Forschungsfragen verbinden, ,die digitale Realität des Computers mit der physischen Realität der Architektur"30 und machen das Digitale fassbar. GKR untersuchen materielle Prozesse, indem sie „Daten und Material, Programmierung und Konstruktion [...] mit der materiellen Realität gebauter Architektur verbinden“".3I

Im Jahr 2012 stellte GKR Mesh Mould vor, das den 3D-Roboterdruck von Betonschalungen mittels thermischer Kunststoffextrusion untersucht. Im Jahr 2016 entwickelte sich die Forschung zu Mesh Mould Metal und dem In situ Fabricator-Demonstrator im Empa NEST DFAB House 20I8. Das ,Mesh" von GKR bezieht sich auf das interne Metallbewehrungssystem, das zur Erhöhung der Zugfestigkeit in Betontrennwänden oder tragenden Wänden verwendet wird: „Das ,Onboard-Sensing - und Berechnungssystem des Roboters ermöglicht es, das Netz ohne externe Messgeräte zu konstruieren. Es orientiert sich am Fundament der Wand, das vor der Roboter-Konstruktion installiert und mit Markern und ,Tags` versehen wird. Eine Kamera am Ende des Effektors misst die Markierungen zur Positionierung des Roboters, und zwei weitere Kameras überwachen die genaue Herstellung des Mesh“.32

Der Agent (Roboter) erscheint als die digitale Version des traditionellen Betonbauers, was jedoch nicht ganz korrekt ist. Die Innovation liegt in der Fähigkeit des Agenten, die Baustelle zu ,meistern', während er die Betonstahlbewehrung in jede gewünschte Form bringt, bevor sie mit Spritzbeton besprüht wird. ${ }^{33}$ GKR-Wände sind keine einfachen orthogonalen Ebenen im Raum und können nahezu jede Form annehmen.

Während der In situ Fabricator ein hohes Maß an Autonomie aufweist, hängt er bei der Erfüllung seiner Aufgabe von der menschlichen Interaktion ab. Die Wand ist vorberechnet, und die Baustelle muss für die Installation des Roboters vorbereitet werden. Allerdings werden ähnliche Maschinen schon bald autonom vor Ort agieren können und in der Lage sein, neue noch nicht entworfene Strukturen zu schaffen. Künstliche Intelligenz und Sensortechnologie bereiten den Weg in diese Zukunft und werden mit hoher Sicherheit einen Paradigmenwechsel in der Entwurfsmethodologie und der Konstruktionstechnik bewirken. 
Adaptive Robotic Carving Giulio Brugnaro und Sean Hanna von der Bartlett School of Architecture, UCL, forschen und entwickeln Methoden zur Konditionierung von Holzverarbeitungssystemen durch Integration von Materialverhalten. Entwurfs- und Fertigungsabläufe vereinen Roboterfertigung, Sensorik und ML, um den Werkprozess zu gestalten. Dabei entlehnen sie MotionTracking-Technologien dem Film-, Entertainment- und Medizinbereich, um die Bewegungsabläufe im Handwerk digital zu erfassen. Ihr ML-Trainingszyklus reicht von der Aufnahme von Kunsthandwerkern bis hin zu autonomen Roboterbewertungen. Eine DesignOberfläche stellt das notwendige Wissen bereit, um Prozesse durch Material- und Werkzeugleistung voranzutreiben. ${ }^{34}$

Brugnaro ,erfindet" das traditionelle Holzbildhauerhandwerk neu, indem er analoge Holzmaserungen und Meißelschnitte mit digitalen Abtast- und Fertigungstechnologien zusammenfügt. Die Forschung zeigt, dass Maschinen in der Lage sind von einem ,Meister' zu lernen und sich dann selbstständig weiterbilden, um neue Handlungen ausführen zu können. Diese Methode stellt klassische lineare Designstrategien in Frage, indem sie Feedbackschleifen zwischen den verschiedenen Phasen des Prozesses einführt. Der Gestalter kann die Prozesse beeinflussen, indem er die Material- und Toolleistung vorgibt, anstatt sie zu bestimmen.

Ausblick Cybercraft vernetzt analoge und digitale MenschMaschine-Interaktion, Sensorik, KI und Materialverhalten, um eine physische Architektur zu verwirklichen. Digitale Architekturmodelle beinhalten eine Vielzahl von Daten. Die gezeigten Anwendungsbeispiele verdeutlichen, dass parametrische digitale Informationsmodelle Robotersysteme und Produktionsabläufe steuern können. Die ,nahtlose“ Interaktion zwischen Mensch, Maschine und Material ermöglicht es, lokal angepasste, maßgefertigte Strukturen zu gestalten und diese in dezentralen Fertigungseinrichtungen sowie vor Ort herzustellen. Diese radikalen Veränderungen der Entwurfsmedien nehmen beständig zu.

Mit Blick auf die Geschichte können wir den Entwicklungsprozess der digitalen Architektur nachvollziehen. Leibniz revolu-

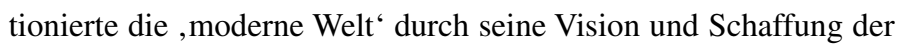
Grundlagen des Binärsystems und der digitalen Rechenmaschine. ${ }^{35}$ Vor Ioo Jahren rief Walter Gropius das Experiment Bauhaus ins Leben, um Architektur im Sinne der modernen Industrialisie- 
rung zu gestalten. Gleichzeitig ließ Karel Čapek den Roboter in die kulturelle Perspektive der Mensch-Maschine einfließen und forderte die Industrialisierung kritisch heraus. Es hat fast 340 Jahre gedauert, bis Leibniz' De Progressione Dyadica und die daraus resultierenden digitalen Medientechnologien mit den physischen Realitäten der Architektur in Einklang gebracht wurden. Wenn Quantencomputer sein theoretisches Versprechen erfüllen, können wir das Binäre schließlich durch ,Qubits ${ }^{6}$ ersetzen. ${ }^{36}$

Wir gingen vom Handcraft zum Digitalcraft über, um zum Cybercraft zu gelangen. Das heißt, die Architektur wandelte sich vom ,Greifbaren` zum , Virtuellen` zum ,Hybriden“ - dem , strukturellen' Material und der ästhetischen Praxis der Architektur. ${ }^{37}$ Cybercraft umfasst unsere einzigartige Fähigkeit, die physische Welt mit Händen und Gedanken zu fassen und erweitert sie durch die digitalen Informationstechnologien. ${ }^{38}$

Barbara Grosz et al. vom I oo Jahre KI-Ausschuss der Standford University glauben ,die zukünftigen Beziehungen der Menschen zu Maschinen werden immer ausgeprägter, fließender und personalisierter werden, da die KI-Systeme lernen, sich an individuelle Persönlichkeiten und Ziele anzupassen. [...] Der Bereich der KI verlagert sich in Richtung des Aufbaus intelligenter Systeme, die effektiv mit Menschen zusammenarbeiten können, einschließlich kreativer Möglichkeiten zur Entwicklung interaktiver und für Menschen angepasster Wege, Roboter zu unterrichten“.39 Roboter, die früher nur von hochqualifizierten Informatikern programmiert werden konnten, sind nun von interdisziplinären Teams für allgemeine Aufgaben programmierbar.

Sensorik, wie CV und ,Force-Torque , vernetzt mit Robotergeräten und KI-Knoten auf ROS, können komplexe Aufgaben bewältigen. Diese Aufgaben reichen von der autonomen Endbearbeitung von Spezialoberflächen durch Scannen von Geometrien in Echtzeit bis hin zu CAD-Knoten, die ästhetische und Materialprozesse beeinflussen. Diese Methoden ermöglichen es ,Meistern', die digitale Materialität physisch zu kontrollieren. ${ }^{40}$

Je mehr wir mit neuen und traditionellen Entwurfsmethoden, Materialien und Fertigungstechnologien interagieren, umso mehr bestimmen sie unsere Wahrnehmung, Interaktion mit, und Gestaltung von Dingen. Die Feedbackschleife zwischen MenschMaschine und Material-Lernen erzeugt eine große Variation von Anwendungsmöglichkeiten. Das Lernen mit Robotern und digital 
informierten Materialien ist ein vielseitiges und bereicherndes Erlebnis. Das Alberti-Paradigma, das Architekten und Architektinnen aus der Materialität der Architektur herauslöst, steht zur Debatte..$^{4}$ Es ist höchste Zeit, dass Architekten und Architektinnen ihre überholte Disziplin und ihre alte Baupraxis neu definieren, indem sie sich kritisch mit diesen neuen Phänomenen befassen.

Informationsbasiertes Modellieren ermöglicht es Architekten und Architektinnen, Fertigungsprozesse von Architekturelementen zunehmend zu steuern. Durch die Entwicklung von Robotik und KI werden Architekten und Architektinnen in der Lage sein, digitale Modelle zu entwerfen, die Material-, Fertigungs- und Montageprozesse verbinden. Traditionelle Handwerksbetriebe können sich schließlich zu lokalen dezentralen Produktionsstätten weiter entwickeln, was zu nachhaltigeren Fertigungs- und Baupraktiken führt. ${ }^{42}$ Diese lokalen Einrichtungen könnten teilautonom architektonische Elemente aus digitalen Dateien herstellen und an nahegelegene Baustellen liefern. Eine Herausforderung kann die robotergestützte Herstellung und Montage von Teilen vor Ort sein. Buchli et al. weisen darauf hin, dass ,die Möglichkeiten bei der Lösung der Herausforderung der ,In-situ-Fertigung ' immens sind. Die Technologie, die diese Aufgabenstellung löst, wird effizientere Prozesse und Produkte in vielen Bereichen ermöglichen, die bisher durch aufwendige Montage und Werkzeugausrüstungen geprägt waren".43 Autonome Maschinen können schließlich unvorhergesehene Konstruktionsprobleme, bei denen Gestalter oft die Kontrolle über den Entwurf verlieren, vor Ort lösen. Architekten und Architektinnen müssen lernen, kritisch mit diesen Möglichkeiten zu experimentieren, um sensible Architekturen zu schaffen, und damit neue Gesellschaftsmodelle zu definieren.

\section{Anmerkungen}

1 Eugène-Emmanuel Viollet-le-Duc, Entretiens sur l'architecture, Paris 1863 , S. 83-84.

2 Bruno Latour, Das terrestrische Manifest, Berlin 2019, S. 88-91.

3 Robin Evans, The Projective Cast - Architecture in Its Three Geometries, Cambridge 1995.

4 Mario Carpo, The Alphabet and the Algorithm, Cambridge 2011, S. 71-79. 
,Robotizierte Konstruktion' bezieht sich in diesem Zusammenhang auf Autonomie und eingebettete Intelligenz, wie sie in der allgemeinen Robotik zu finden sind.

6 Siehe dazu David Pye, The Nature and Art of Workmanship, London 1968, S. 20: „Wenn ich dem Wort Handwerkskunst eine Bedeutung zuschreiben muss, möchte ich als erste Annäherung sagen, dass es sich um eine einfache Handwerkskunst mit irgendeiner Art von Technik oder Apparatur handelt, bei der die Qualität des Ergebnisses nicht vorgegeben ist, sondern von dem Urteilsvermögen, der Geschicklichkeit und der Sorgfalt abhängt, die der Hersteller während seiner Arbeit anwendet. Die wesentliche Idee ist, dass die Qualität des Ergebnisses während des Herstellungsprozesses ständig gefährdet ist; deshalb nenne ich diese Art der Verarbeitung ,Die Verarbeitung des Risikos': eine ungeschliffene Phrase, aber zumindest beschreibend".

7 George Chaikin, "The Computer and the Studio", EAAE/eCAADe International Workshop Proceedings, Leuven 1998, S. 51-54.

8 "Leichte, durchsichtige, gleichermaßen schützende, wie offene Dächer überspannen den sanft modellierten Olympiapark mit Olympiastadion, Olympiahalle und Olympiaschwimmhalle. Eine Seilnetzkonstruktion aus vielen fast regelmäßigen sattelförmigen, mit Randseilen eingefassten Flächen ist an mehreren Punkten von außenstehenden Masten abgehängt, beziehungsweise von innen durch sogenannte Luftstützen getragen und nach außen abgespannt. Völlig neuartig waren die Anforderungen, die dieses Dach an die geometrische Genauigkeit, sowohl hinsichtlich des Zuschnittes als auch der Vorfertigung stellte. So kam es hier erstmals zu einem Computereinsatz in großem Stile für derartige Aufgaben und - für die spätere ,High-Tech-Architektur' von besonderer Bedeutung - zu einer Wiedergeburt des Gussstahles im Bauwesen". www.sbp.de/projekt/dach-olympiastadion-muenchen-1972-1/ [14.08.2019].

9 In CAD verschiedene Farben stellten unterschiedliche Liniengewichte dar; Architekten und Architektinnen wurden geschickt, ihre Zeichnungen durch Farbe zu lesen, bevor sie sie plotten - eine bizarre Aufgabe, die bis heute besteht.

10 Marshall McLuhan, Understanding Media: The Extensions of Man, London 1974.

11 Wie im Beitrag von Leon Batista Alberti, Philibert de L’Orme, Gaspar Monge, Walter Gropius, Frank Gehry.

12 Während der 1990er Jahre entstanden neue Schulen und Architekturausbildungsprogramme, wie die "Paperless Studios" der Columbia University GSAPP, SCI-ARC, Die Angewandte und 
AA London. Ihre Kernagenda untersuchte entstehende digitale Medientechnologien als performative Medien für kreativen Ausdruck und kritische Reflexion.

13 Greg Lynn, Animate Form, New York 1999.

14 „Smartgeometry (Sg) wurde 2001 als Partnerschaft zwischen Praxis, Forschung und Wissenschaft gegründet, bestehend aus Mitgliedern der weltweit führenden Architektur- und Ingenieurbüros und Bildungseinrichtungen. Für die neuen Generationen von Designern, Ingenieuren und Architekten werden Mathematik und Algorithmen so natürlich wie Stift und Bleistift. Die Sg fördert die Entstehung dieses neuen Paradigmas, in dem Digital Designer und Handwerker die Kombination von digitalen und physischen Medien intelligent nutzen können, um Projekte vom Design bis zur Produktion zu begleiten". www.smartgeometry.org/about/ [14.08.2019].

15 Paola Antonelli, Design and the Elastic Mind, New York 2008.

16 „Advances in Architectural Geometry (AAG) ist eine Konferenz, auf der sowohl theoretische als auch praktische Arbeiten im Zusammenhang mit neuen geometrischen Entwicklungen vorgestellt werden. Daran sind Architekten, Ingenieure, Informatiker, Mathematiker, Software- und Algorithmen-Designer und -Auftragnehmer beteiligt. Unser Ziel ist es, Forscher aus Architektur- und Ingenieurbüros, Hochschulen und der Industrie zusammenzubringen. Die AAG hat sich zu einer Referenz in Forschung und Praxis entwickelt und wird durch die direkte Beteiligung der renommiertesten Architekturund Ingenieurbüros sowie der Forschungslabors unterstützt". www. architecturalgeometry.org/aag18/ [14.08.2019].

17 Christophe Barlieb, "Architectonics", in: Vera Viana, Alexandra Paio (Hg.), Geometrias'14 New Challenges on Practice, Researching and Teaching Geometries and Drawing, Lissabon 2014, S. 139-145.

18 Horizon 2020, Building Innovation in the Extended Digital Chain, InnoChain, www.cordis.europa.eu/project/rcn/193957/results/de [14.08.2019].

19 Mies van der Rohe war auf der Suche nach architektonischen Erkenntnissen. Er nutzte grundlegend standardisierte industrielle Materialeigenschaften (Walzstahl und Glas) durch präzise ,strukturelle' (wie in der Grammatik) Detaillierung. Le Corbusier strebte nach einer neuen Architektur, einem ,neuen Geist'. Auch er ließ sich von der Technisierung und dem funktionalen Formalismus inspirieren: Boote, Flugzeuge und Autos. Das Domino-Haus war ein direktes Ergebnis der mit der Normung verbundenen strukturellen Klarheit und führte zu seinem berühmten Anspruch: „Das Haus ist eine Maschine zum Leben". Die Werkzeuge, mit denen diese architektonischen ,Modelle' hergestellt wurden, reichen jedoch bis in die 
Renaissance zurück. Le Corbusier ging mit Ronchamp neue Wege, aber die Einschränkungen der Darstellenden Geometrie begrenzten sie einigermaßen.

20 Jan Willmann, Philippe Block, Marco Hutter, Kendra Byrne, Tim Schork (Hg.), Robotic Fabrication in Architecture, Art and Design 2018, Cham 2018. S. 9-13.

21 The Science of Intelligence. www.scienceofintelligence.de/about-us/ our-mission/ [14.08.2019].

22 Earth. www.earth.nullschool.net/ [14.08.2019].

23 SmartCitizen. www.smartcitizen.me/kits/ [14.08.2019].

24 ROS. www.ros.org [14.08.2019].

25 SARA. www.dlr.de/rm/desktopdefault.aspx/tabid11709/\#gallery/29681 [14.08.2019].

26 Wojciech Czaja, „Architekturtage 2019: Ein Baustoff namens Klima“, Der Standard, 20.05.2019, www.derstandard.at/ story/2000103499653/ein-baustoff-namens-klima [14.08.2019].

27 Philippe Rahm, Convective Apartments, Hamburg 2010, www.philipperahm.com/data/projects/convectiveapartments/ [14.08.2019].

28 Alex Tieghi-Walker, „Philippe Rahm's Holistic Décor in the Age of Climate Change", PIN-UP, www.pinupmagazine.org/articles/philippe-rahm [14.08.2019].

29 Gramazio Kohler, The Robotic Touch, Zürich 2014, S. 218-131.

30 Ebd., S. 9.

31 Ebd., S. 15.

32 Gramazio Kohler Research. www.gramaziokohler.arch.ethz.ch/ web/e/forschung/324.html [14.08.2019].

33 Jonas Buchli et al., "Digital in situ fabrication - Challenges and opportunities for robotic in situ fabrication in architecture, construction and beyond", in Robert Flatt, Timothy Wangler (Hg.), Cement and Concrete Research, Lausanne 2018, S. 70-75.

34 Giulio Brugnaro, Sean Hanna, "Adaptive Robotic Carving, Training Methods for the Integration of Material Performances in Timber Manufacturing" in: Jan Willmann, Philippe Block, Marco Hutter, Kendra Byrne, Tim Schork (Hg.), Robotic Fabrication in Architecture, Art and Design 2018, Cham 2018. S. 336-348.

35 Hans Poser, "Die Schöpfung der Welt aus Null und Eins. Gottfried Wilhelm Leibniz im Porträt", Der Blaue Reiter - Journal für Philosophie 41 - Die Seele im Digitalen Zeitalter, 2018, S. 96-101.

36 Cade Metz, „Google Claims a Quantum Breakthrough That Could Change Computing", New York Times, 23.10.2019. www.nytimes. 
com/2019/10/23/technology/quantum-computing-google.html [23.10.2019].

37 Das „Strukturelle" kann in den Formulierungen von Mies van der Rohe verstanden werden: „Dann wurde mir klar, dass es nicht Aufgabe der Architektur war, die Form zu erfinden. Ich versuchte zu verstehen, was diese Aufgabe war. Ich habe Peter Behrens gefragt, aber er konnte mir keine Antwort geben. Diese Frage stellte er nicht. Die anderen sagten: ,Was wir bauen, ist Architektur', aber wir waren mit dieser Antwort nicht zufrieden.... Da wir wussten, dass es eine Frage der Wahrheit ist, versuchten wir herauszufinden, was Wahrheit wirklich ist. Wir waren sehr erfreut, die Definition der Wahrheit von St. Thomas von Aquin zu finden: ,Adequatio intellectus et rei', oder wie ein moderner Philosoph es in der Sprache von heute ausdrückt: ,Wahrheit ist die Bedeutung der Tatsache'. Berlag war ein Mann von großer Ernsthaftigkeit, der nichts akzeptieren würde, was gefälscht war, und er war es, der sagte, dass nichts gebaut werden sollte, was nicht klar konstruiert ist. Und Berlag tat genau das. Und das in einem Ausmaß, das in Amsterdam berühmt ist. Der Beuis, war ein mittelalterlicher Charakter, ohne mittelalterlich zu sein. Er benutzte Ziegel, wie es die mittelalterlichen Menschen taten. Die Idee einer klaren Konstruktion kam mir dort als eine der Grundlagen, die wir akzeptieren sollten. Wir können leicht darüber reden, aber es ist nicht einfach, es zu tun. Es ist sehr schwierig, an dieser grundlegenden Konstruktion festzuhalten und sie dann zu einer Struktur zu erheben. Ich muss klarstellen, dass man in der englischen Sprache alles strukturiert nennt. In Europa tun wir das nicht. Wir nennen als Hütte eine Hütte und nicht eine Struktur. Nach der Struktur haben wir eine philosophische Idee. Die Struktur ist das Ganze von oben nach unten, bis ins letzte Detail mit der gleichen Idee. Das ist es, was wir Struktur nennen. (Zitat von Peter Carter in der Architektur, März 1961)“. Kenneth Frampton, Moderne Architecture, New York 1980, S. 161.

38 Otl Aicher, Analog und Digital, Berlin 2015, S. 21-26.

39 Barbara Grosz et al., Artificial Intelligence and Life in 2030, One Hundred Year Study on Artificial Intelligence, Report of the 2015 Study Panel, Stanford 2016, S. 6.

40 Dies ist ein großer Unterschied zu 3D-Drucktechnologien, die aufgrund von Materialschichten virtuelle Materialeigenschaften aufweisen. Nur bei der Materialfusion ,Selective Laser Sintering' (SLS) kehren additive Technologien in den physikalischen Bereich der Architektur zurück. Standard 3D-Drucktechnologien können für Gießzwecke, wie z.B. Formen, nützlich sein, aber ihre Materi- 
aleigenschaften sind nur für diesen Zweck geeignet. Die Idee von 3D-Druckhäusern bleibt aus zahlreichen Gründen fragwürdig, die in diesem Beitrag nicht näher erläutert werden.

41 Mario Carpo, The Alphabet and the Algorithm, Cambridge, MA. 2011, S. 44-48.

42 Matthias Böttger, Stefan Carsten, Ludwig Engel, Speculations Transformations, Zürich 2016, S. 107-108.

43 Buchli et al., "Digital in situ fabrication" (Anm. 33), S. 67. 



\section{Bildnachweise}

\section{Kassandra Nakas - Manifeste, Diskurse, Chatter}

I Carlo Ratti Associati, Open Source Architecture Manifesto, Installationsansicht Istanbul Biennale 20 I 2, credits: carlorattiassociati I walter nicolino \& carlo rattiTeam: Carlo Ratti, Pietro Leoni, Antonio Atripaldi, Giovanni de Niederhausern, Enrico Gueli, Franco Magni, Walter Nicolino. Special thanks to Officine Arduino / FabLab Torino.

2 Bureau Spectacular/Jimenez Lai, The Politics of Flatness, Graham Foundation, Chicago, 20 I5, credits: Bureau Spectacular / Jimenez Lai.

\section{Carolin Höfler - Über die Wirkmacht der Linie}

I Courtesy of The Zaha Hadid Foundation.

2 (C) Harvard Art Museums / Arthur M. Sackler Museum, The Edwin Binney, 3rd Collection of Turkish Art at the Harvard Art Museums, Image Number: DDC254207, Accession Number: I985.257. Foto: (C) President and Fellows of Harvard College.

3 Gabriel Rollenhagen, Selectorum emblematum centuria secunda, Ultraiecti [Utrecht] I6I3, gebunden mit einer Kopie des ersten Teils der Arbeit: Gabriel Rollenhagen, Nucleus emblematum selectissimorum. Köln I6 I , 24. Emblem, unter: www.archive.org/details/gabrielisrollenhooroll, S. 75 [o I. Juni 20I9]. Los Angeles, Getty Research Institute.

4 Courtesy of The Zaha Hadid Foundation.

5 Musée d'art et d'histoire de Genève, Trésors de l'Islam (Hg.), Calligraphie islamique. Textes sacrés et profanes / Islamic Calligraphy. Sacred and Secular Writings, Ausst.kat. (Genf, Musée d'art et d'histoire, I988), Genf I988, S. 55.

6 Courtesy of The Zaha Hadid Foundation. 
7 William Hogarth, The Analysis of Beauty. Written with a View of Fixing the Fluctuating Ideas of Taste, London I753, unter: www.archive.org/details/analysisofbeautyoohoga [o I. Juni 20I9]. Los Angeles, Getty Research Institute.

8 Wassily Kandinsky, Punkt und Linie zu Fläche. Beitrag zur Analyse der malerischen Elemente, Bauhausbücher, Bd. 9, München I926, S. 8I-82, Fig. 44-46.

9 (C) LWL-Museum für Kunst und Kultur, Westfälisches Landesmuseum, Münster / Sammlung Cremer, Inv.Nr. KdZ 3520 LM. Fotoå: (C) LWL-Museum für Kunst und Kultur, Westfälisches Landesmuseum, Münster / Hanna Neander.

IO-I2 Courtesy of The Zaha Hadid Foundation.

I3 Courtesy of Zaha Hadid Architects.

I4 (C) Mehmed Özçay, unter: www.ozcay.com/mehmed/galeri\#62 [or. Juni 20I9]. Dr. Irvin C. Shick Collection, Boston.

I 5 Courtesy of Zaha Hadid Architects.

I6 Unter: www.commons.wikimedia.org/wiki/File:Alif-individua-cropt.svg und www.commons.wikimedia.org/wiki/ File: I8-Ain.svg\#/media/File: I 8-Ain.svg [o . Juni 20 I9].

I7 Schispringen Bergisel - Austria HD Travel Channel, 20 I I, unter: https://www.youtube.com/watch?v=UartmuQ1_Vk [OI. Juni 20I9].

\section{Anna Hougaard - Skizzen von Diagrammen und Diagramme von Skizzen}

Alle Abbildungen wurden von der Autorin erstellt.

\section{Philipp Reinfeld - Fotografie und Entwerfen}

I Claude-Glas, Ausschnitt, Science Museum / Science and Society Picture Library. (C) iPhone 5 in Lederschutzhülle der Firma Borofone, Autor unbekannt.

2 Piero della Francesca, De Prospectiva Pingendi, ca. I475. I Albrecht Dürer, Underweysung der Messung, mit dem Zirckel und Richtscheyt in Linien, Ebenen und gantzen corporen, Nüremberg, I525, SLUB Dresden, S. I 8 I.

3 Laura Brüggemann, Janna Hinzpeter, Institut für Mediales Entwerfen, Department Architektur, TU Braunschweig.

4 Ezra Stoller, Fallingwater, Frank Lloyd Wright, Bear Run, PA, I97I.

5 Google-Bildersuche [06.20 I5]. 
M. Seitz / Richard Szeliski, Building Rome in a Day, 200920 I I: www.grail.cs.washington.edu/rome/, [ I0.20I3].

7 Agarwal/Furukawa/Snavely/Curless/Seitz/Szeliski, RECONSTRUCTING ROME, S. 46: www.cs.cornell.edu/ snavely/ publications/papers/ieee_computer_rome.pdf.

8 Keith N. Snavely, Scene Reconstruction and Visualization from Internet Photo Collections, Doctoral thesis, University of Washington, 2008, S. 88.

9 Ebd., Figure 3.4, S. 34

Io, I I Studienprojekt Hannah Linck, Institut für Mediales Entwerfen, Department Architektur, TU Braunschweig.

I Foto Philipp Reinfeld, Studienprojekt, Institut für Mediales Entwerfen, Department Architektur, TU Braunschweig.

\section{Matthias Ballestrem - Experimentelles Entwerfen}

I Joe Fig, Pollock (2008), 20 x 53 x $44 \mathrm{~cm}$, mixed media. (C) The Artist and Cristin Tierney Gallery, New York, NY.

2, 3 (C) Katja Hentschel.

4-6 (C) Matthias Ballestrem.

7 C Ensamble Studio.

\section{Norbert Palz - Architektonischer Entwurf und differenzierte}

\section{Mediennutzung}

I Christopher Alexander, Diagramm aus „A City is not a Tree“, Architectural Forum, 1965, I: S. 58-62.

2, 3 C Sergio González / ETH Zürich, Block Research Group 2014

4 Alle Bilder aus Camille Lacadee, François Roche, Vongsawat Wongkijjalerd und Daniela Mitterberger, concrete[i]land. Edited by new-territories / R\&Sie(n), 2015: www.new-territories.com/blog/?p=2 I 6 I [28.08.20 I6]. (C) New-Territories, M4, with Innsbruck University, François Roche with Camille Lacadee and Stephan Henrich.

5, 6 Sean Ahlquist, Leah Ketcheson und Costanza Colombi, „Multisensory Architecture: The Dynamic Interplay of Environment, Movement and Social Function“", AD-Architecural design, 2017. (C) Sean Ahlquist, University of Michigan

\section{Corneel Cannaerts - Hacking Agency}

Alle Abbildungen wurden vom Autor erstellt. 



\section{AutorInnen}

Matthias Ballestrem ist Architekt und seit 2017 Professor für Architektur und experimentelles Entwerfen an der HCU in Hamburg. Seit 2006 hatte er verschiedene Positionen in der Entwurfslehre inne unter anderem an der Cornell Universität, im GAD Berlin Program des CIEE und an der TU Berlin, wo er zuletzt von 20I3-I 8 als Gastprofessor lehrte.

Christophe Barlieb ist Architekt und spezialisiert auf Architekturmedien. Er beschäftigt sich mit der Entwicklung angewandter Robotik in der Architektur. Sein Beitrag zur Robotik im Handwerk wurde 20 I9 mit dem Bayerischen Staatspreis für Innovation ausgezeichnet. Er war unter anderem Gastprofessor an der TU Berlin und als wissenschaftlicher Mitarbeiter an der TU Braunschweig tätig.

Corneel Cannaerts ist Architekt und Post-Doc-Forscher, spezialisiert auf die Wirkung neuer Technologien auf die Kultur und Praxis der Architektur. Er besitzt einen Master in Architectural Engineering an der Universität Gent und einen PhD in Architektur bei der SIAL/ RMIT University of Melbourne. Er forscht und lehrt an der Fakultät für Architektur der KU Leuven.

Ana García Varas ist Professorin für Ästhetik am Institut für Philosophie der Universität Zaragoza (Spanien). Ihre gegenwärtigen Forschungsinteressen konzentrieren sich auf die Analyse von Formen von Agency im Kontext ästhetischer Praktiken. Sie ist die Leiterin des nationalen Forschungsprojekts „Bilder, Handeln und Macht: Ikonische ,Agency“ und zeitgenössische Praktiken“. 
Lidia Gasperoni ist Philosophin und forscht $\mathrm{zu}$ Architekturtheorie, Medienphilosophie und Ästhetik. Sie ist wissenschaftliche Mitarbeiterin in der Post-Doc-Phase am Fachgebiet für Architekturtheorie der TU Berlin. Ihr besonderes Interesse gilt der Verbindung von Theorie und Praxis durch die Erforschung experimenteller Praktiken und die Konzipierung von Ausstellungen architektonischer Entwurfsprozesse.

Anna Hougaard ist Architektin und Forscherin an der TU Berlin, Institut für Architektur, Fachgebiet CODE. Sie hat an der KADK promoviert. Sie ist spezialisiert auf Architekturzeichnung, Diagrammatik und künstlerische Forschung in der Architektur.

Carolin Höfler ist Kunsthistorikerin und Architektin. Seit 20I3 ist sie Professorin für Designtheorie und -forschung an der Technischen Hochschule Köln. Sie hat an der Humboldt-Universität zu Berlin promoviert. Als Sprecherin der Forschungsstelle „Echtzeitstadt“ der TH Köln untersucht sie urbane Bildumwelten und das Zusammenspiel von Körpern und technischen Dingen in öffentlichen Räumen.

Kassandra Nakas ist Kunstwissenschaftlerin und lehrt derzeit am IMD Institute of Media and Design der TU Braunschweig. Sie hat an der Freien Universität Berlin promoviert. Sie hatte eine Gastprofessur an der Universität der Künste Berlin inne und hat an der Leuphana Universität Lüneburg und der Köln International School of Design (KISD) gelehrt.

Norbert Palz ist ein deutscher Architekt und Hochschullehrer. Nach Bauzeichnerlehre und Architekturstudium an der TU Berlin arbeitete er von I999-2002 bei UN Studio in Amsterdam und NOX Architects in Rotterdam und gründete 2003 mit Robert Banovic das Büro TARGADESIGN. 20 I 2 folgte eine Promotion an der KADK zu additiven Fertigungsmethoden in der Architektur. Seit 20 Io ist Professor für „Digitales und Experimentelles Entwerfen“ an der Universität der Künste in Berlin. Seit 2020 ist er Präsident der UdK Berlin.

Philipp Reinfeld forscht $\mathrm{zu}$ den Auswirkungen bildlich-medialer Raumrepräsentation auf die Rezeption und Produktion von Architektur und Stadt. 20I6 promovierte er am Lehrstuhl für Architekturtheorie der Universität Innsbruck. Er ist stellvertretender Leiter des Institute of Media and Design am Department Architektur der TU Braunschweig. 


\section{Architektur Denken}

1 Architekturtheorie heute. Jörg H. Gleiter, 2008

ISBN 978-3-89942-879-7

2 Die enzyklopädische Architektur. Gerd de Bruyn, 2008 ISBN 978-3-89942-984-8

3 Welten und Gegenwelten. Arata Isozaki, 2011

Übersetzt und herausgegeben von Yoco Fukuda, Jörg H. Gleiter und Jörg R. Noennig

ISBN 978-3-8376-1116-8

4 Urgeschichte der Moderne. Jörg H. Gleiter, 2010 ISBN 978-3-8376-1534-0

5 Das Wissen der Architektur. Gerd de Bruyn, Wolf Reuter, 2011 ISBN 978-3-8376-1553-1

6 Alphabet und Algorithmus. Mario Carpo, 2012 Herausgegeben von Jörg H. Gleiter, aus dem Englischen übersetzt von Jan Bovelet und Jörg H. Gleiter ISBN 978-3-8376-1355-1

7 Symptom Design. Herausgegeben von Jörg H. Gleiter, 2014 ISBN 978-3-8376-2268-3

8 Architektur und Philosophie. Herausgegeben von Jörg H. Gleiter und Ludger Schwarte, 2015 ISBN 978-3-8376-2464-9

9 Das Diaphane. Herausgegeben von Ulrike Kuch, 2020 ISBN 978-3-8376-4282-7

10 Media Agency. Herausgegeben von Christophe Barlieb und Lidia Gasperoni, 2020

ISBN 978-3-8376-4874-4

Erhältlich im Buchhandel oder beim transcript Verlag. Portofreie Zustellung bei Bestellung direkt vom Verlag. Fax +49 (521) 393797-34 bestellung@transcript-verlag.de www.transcript-verlag.de 
